University of Tennessee Health Science Center

UTHSC Digital Commons

\title{
Treatment Effects of the Mandibular Anterior Repositioning Appliance (MARA) and Conventional Orthodontic Mechanics on Temporomandibular Joint Morphology: A Comparison Using Cone Beam Computed Tomography
}

Brenton E. Glassell

University of Tennessee Health Science Center

Follow this and additional works at: https://dc.uthsc.edu/dissertations

Part of the Orthodontics and Orthodontology Commons

\section{Recommended Citation}

Glassell, Brenton E. , "Treatment Effects of the Mandibular Anterior Repositioning Appliance (MARA) and Conventional Orthodontic Mechanics on Temporomandibular Joint Morphology: A Comparison Using Cone Beam Computed Tomography" (2013). Theses and Dissertations (ETD). Paper 102.

http://dx.doi.org/10.21007/etd.cghs.2013.0112.

This Thesis is brought to you for free and open access by the College of Graduate Health Sciences at UTHSC Digital Commons. It has been accepted for inclusion in Theses and Dissertations (ETD) by an authorized administrator of UTHSC Digital Commons. For more information, please contact jwelch30@uthsc.edu. 


\title{
Treatment Effects of the Mandibular Anterior Repositioning Appliance (MARA) and Conventional Orthodontic Mechanics on Temporomandibular Joint Morphology: A Comparison Using Cone Beam Computed Tomography
}

\begin{abstract}
Introduction: An ongoing debate exists in orthodontics about the amount of condylefossa remodeling and growth seen in Class II patients using functional appliance therapy. Some clinicians and researchers have found that that the use of functional appliances increases the amount of condylar growth and subsequent Class II skeletal correction; others believe that the mandible has a preset amount of growth and that conventional orthodontics will result in a similar amount of condylar growth to functional appliance therapy. Purpose: The aim of this study was to compare condylar remodeling in functional appliance treatment using the mandibular anterior repositioning appliance (MARA) to conventional fixed orthodontic therapy by measuring changes using Cone Beam Computed Tomography (CBCT).

Methods: This retrospective study was an analysis of cone beam computed tomography images taken on 65 subjects with Class II division 1 malocclusions at the beginning and end of their comprehensive orthodontic treatment. Thirty-three subjects ( 13 females, 20 males; average age 11.93 years) were treated with the MARA plus edgewise mechanics, and a sample of thirtyone subjects ( 18 females, 13 males; average age 12.37 years) were treated with full fixed conventional orthodontic appliances and class II intermaxillary elastics. The CBCT scans were evaluated using Dolphin3D@. Temporomandibular joint (TMJ) measurements were recorded in the sagittal, axial, and coronal axes, and lateral cephalograms were extracted to compare groups and evaluate treatment differences. A total of 98 variables were measured.

Results: Slightly more mandibular advancement was seen in the MARA group versus the conventional group. In looking at the TMJ dimensions, the joint spaces were relatively constant throughout treatment, but more resorption of the condylar head was observed in the MARA group versus conventional treatment. The condylar head angle decreased for both treatment groups, showing a forward remodeling. Lengthening of the condylar process was statistically greater in the MARA group than the conventional Edgewise treatment group, although both increased on average. The slope of the posterior border of the articular eminence became more acute in the MARA group and more obtuse in the conventional Edgewise group. Overall, there was a relative increase and forward advancement of the temporomandibular apparatus in both treatment groups.
\end{abstract}

Conclusions: Both treatment groups were successful in correcting the Class II malocclusion skeletally and dentally. However, the MARA group had slightly more mandibular advancement and condylar growth than the conventional treatment group. In both groups, the TMJ structures increased and came downward and forward partly due to treatment and, to some extent, growth in the adolescent subjects.

\section{Document Type}

Thesis

\section{Degree Name}

Master of Dental Science (MDS)

\section{Program}

Orthodontics 


\section{Research Advisor}

Edward F. Harris, Ph.D.

\section{Keywords}

CBCT, Class II, Functional, MARA, Orthodontic, TMJ

\section{Subject Categories}

Dentistry | Medicine and Health Sciences | Orthodontics and Orthodontology 


\title{
Treatment Effects of the Mandibular Anterior Repositioning Appliance (MARA)
} and Conventional Orthodontic Mechanics on Temporomandibular Joint Morphology: A Comparison Using Cone Beam Computed Tomography

\author{
A Thesis \\ Presented for \\ The Graduate Studies Council \\ The University of Tennessee \\ Health Science Center
}

\author{
In Partial Fulfillment \\ Of the Requirements for the Degree \\ Master of Dental Science \\ From The University of Tennessee
}

By

Brenton E. Glassell

May 2013 
Copyright (C) 2013 by Brenton E. Glassell. All rights reserved. 


\section{ACKNOWLEDGEMENTS}

I would like to thank Dr. Edward Harris for his expertise, guidance and inspiration. Under his direction, I gained an enthusiasm for the research process and valuable experience that I will apply throughout my career. I would also like to thank Dr. Dan Merwin and Dr. Bill Parris for serving on my thesis committee. Their thoughtful insight and support were invaluable throughout this process. I would also like to thank Dr. Ken Dillehay, Dr. Dan Merwin, and Dr. Preston Miller for allowing me access to their CBCT images. I would also like to thank my parents, Edwin and Deborah Glassell, for all their support through my academic years and their teaching me hard work and instilling in me a thirst for knowledge. Lastly, and most of all, I would like to thank my beautiful bride Gail for all her support during this 3-year project. 


\begin{abstract}
Introduction: An ongoing debate exists in orthodontics about the amount of condyle-fossa remodeling and growth seen in Class II patients using functional appliance therapy. Some clinicians and researchers have found that that the use of functional appliances increases the amount of condylar growth and subsequent Class II skeletal correction; others believe that the mandible has a preset amount of growth and that conventional orthodontics will result in a similar amount of condylar growth to functional appliance therapy.
\end{abstract}

Purpose: The aim of this study was to compare condylar remodeling in functional appliance treatment using the mandibular anterior repositioning appliance (MARA) to conventional fixed orthodontic therapy by measuring changes using Cone Beam Computed Tomography (CBCT).

Methods: This retrospective study was an analysis of cone beam computed tomography images taken on 65 subjects with Class II division 1 malocclusions at the beginning and end of their comprehensive orthodontic treatment. Thirty-three subjects (13 females, 20 males; average age 11.93 years) were treated with the MARA plus edgewise mechanics, and a sample of thirty-one subjects (18 females, 13 males; average age 12.37 years) were treated with full fixed conventional orthodontic appliances and class II intermaxillary elastics. The CBCT scans were evaluated using Dolphin $3 \mathrm{D}^{\odot}$. Temporomandibular joint (TMJ) measurements were recorded in the sagittal, axial, and coronal axes, and lateral cephalograms were extracted to compare groups and evaluate treatment differences. A total of 98 variables were measured.

Results: Slightly more mandibular advancement was seen in the MARA group versus the conventional group. In looking at the TMJ dimensions, the joint spaces were relatively constant throughout treatment, but more resorption of the condylar head was observed in the MARA group versus conventional treatment. The condylar head angle decreased for both treatment groups, showing a forward remodeling. Lengthening of the condylar process was statistically greater in the MARA group than the conventional Edgewise treatment group, although both increased on average. The slope of the posterior border of the articular eminence became more acute in the MARA group and more obtuse in the conventional Edgewise group. Overall, there was a relative increase and forward advancement of the temporomandibular apparatus in both treatment groups.

Conclusions: Both treatment groups were successful in correcting the Class II malocclusion skeletally and dentally. However, the MARA group had slightly more mandibular advancement and condylar growth than the conventional treatment group. In both groups, the TMJ structures increased and came downward and forward partly due to treatment and, to some extent, growth in the adolescent subjects. 


\section{TABLE OF CONTENTS}

CHAPTER 1. INTRODUCTION ....................................................................................

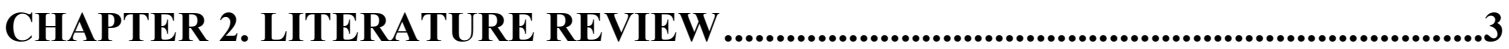

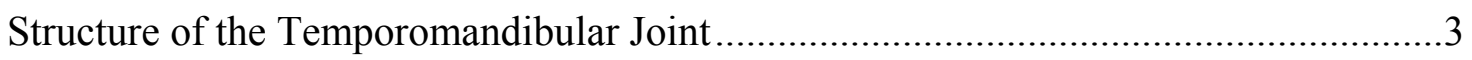

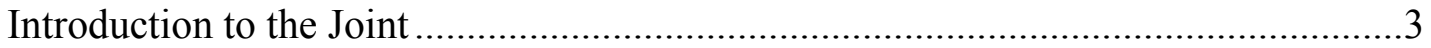

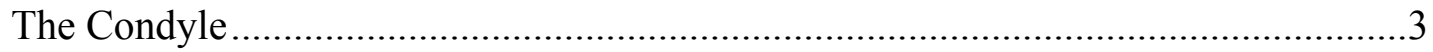

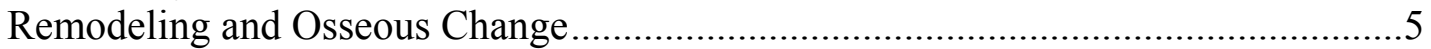

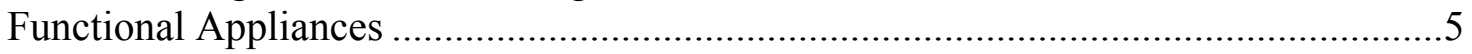

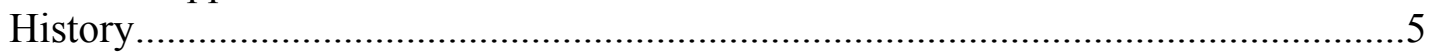

Mandibular Anterior Repositioning Appliance ………………………………….....6

Temporomandibular Changes with Functional Appliances............................................8

Proponents of Condylar Remodeling and Modified Growth .......................................8

Opponents of Functional Appliance Therapy ……………...................................10

Cone Beam Computed Tomography …………………............................................12

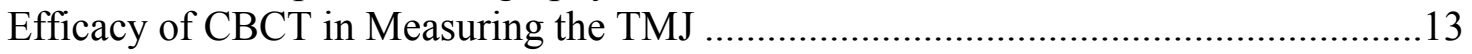

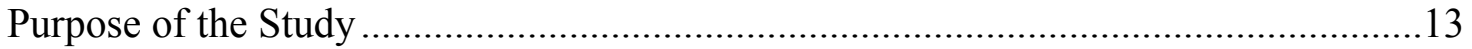

CHAPTER 3. MATERIALS AND METHODS...........................................................15

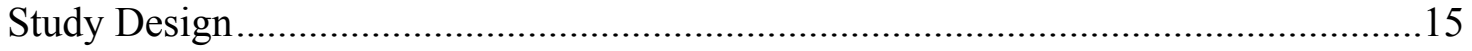

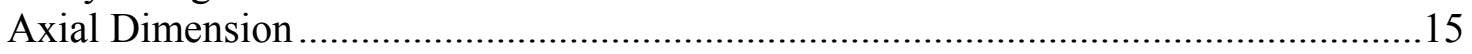

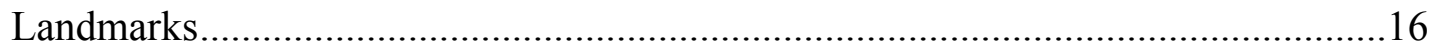

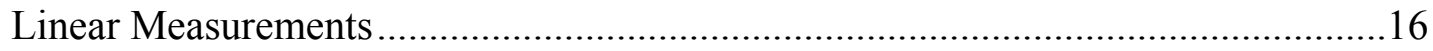

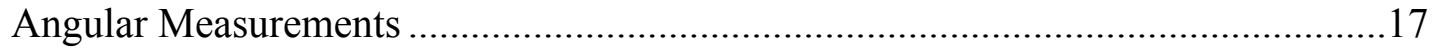

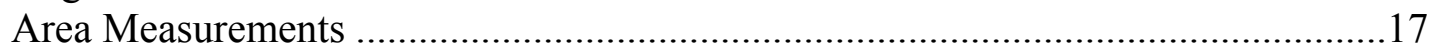

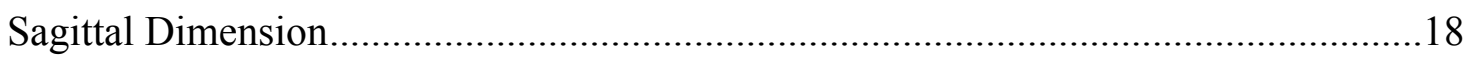

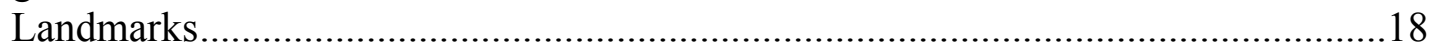

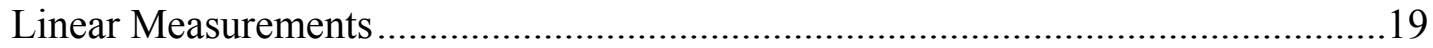

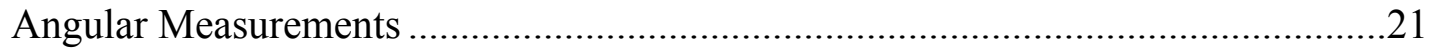

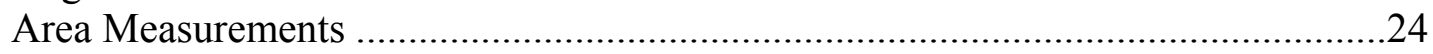

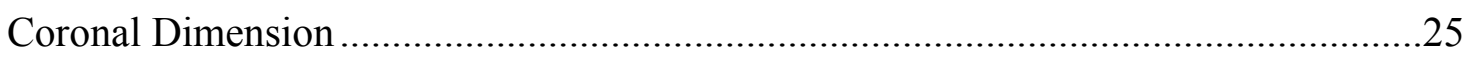

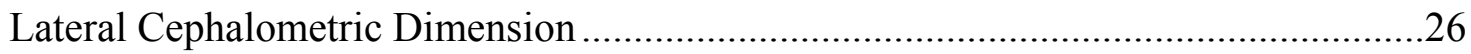

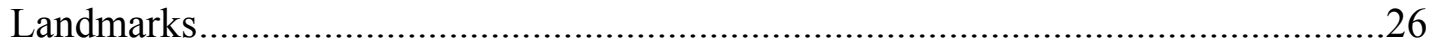

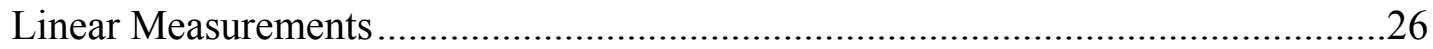

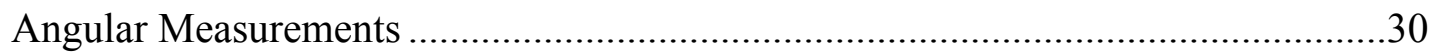

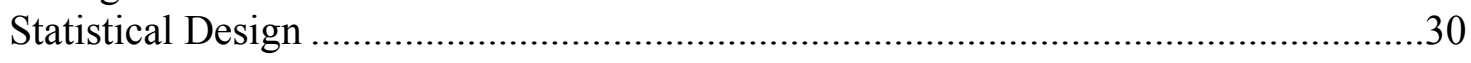

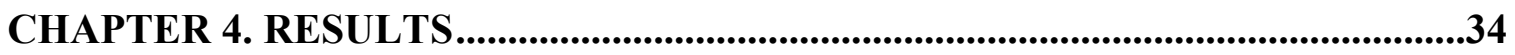

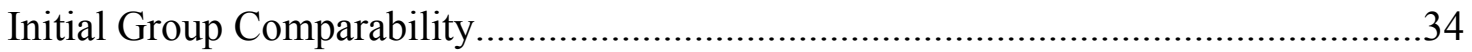

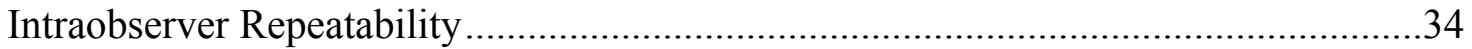

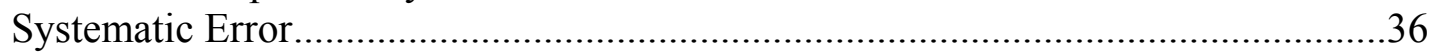

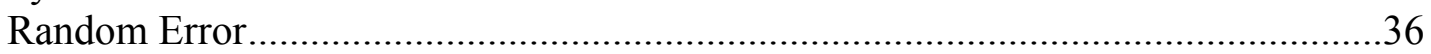

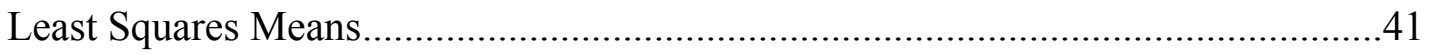

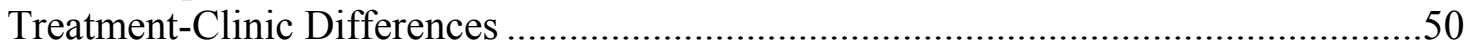


CHAPTER 5. DISCUSSION ..........................................................................................74

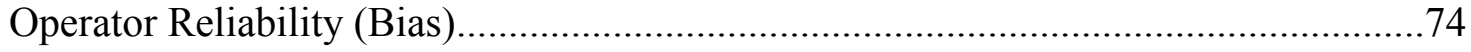

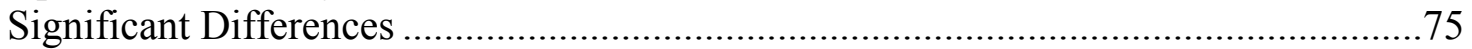

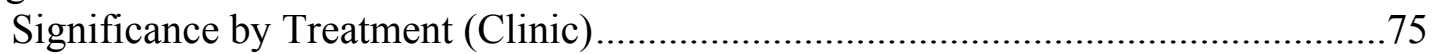

Side-by-Treatment Significant Differences .........................................................78

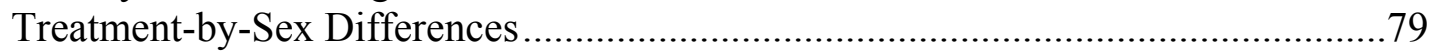

Treatment Differences Seen in Both Groups ........................................................79

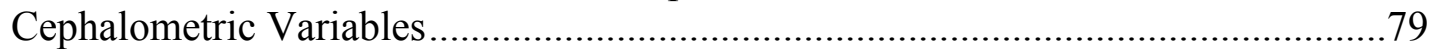

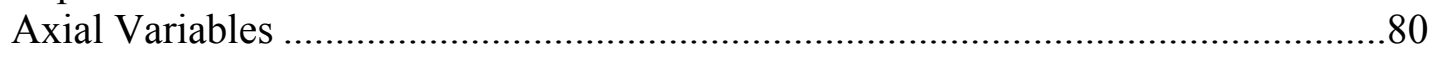

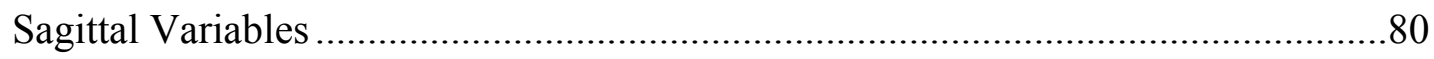

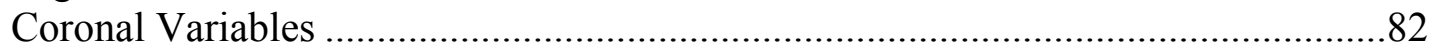

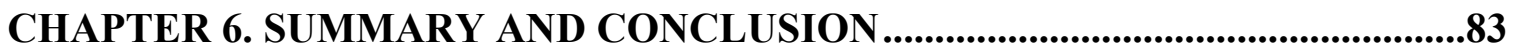

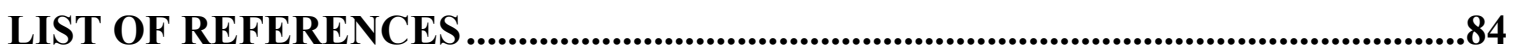

APPENDIX A. DESCRPTIVE STATISTICAL RESULTS FOR EACH

VARIABLE, PARTITIONED BY TREATMENT PLUS ONE-SAMPLE T-

TESTS ASSESSING THE IN-TREATMENT CHANGES

APPENDIX B. RESULTS OF ANALYSIS OF VARIANCE FOR TREATMENT OR SEX DIFFERENCES IN THE UNILATERAL (MIDLINE) DIMENSIONS AND TREATMENT, SEX, AND SIDE (LEFT, RIGHT) IN THE BILATERAL DIMENSIONS.

APPENDIX C. LEAST-SQUARES MEANS FOR THE VARIABLES, PARTITIONED BY TREATMENT, SEX, AND TREATMENT-BY-SEX

APPENDIX D. RESULTS OF PAIRED T-TESTS ASSESSING FOR SYSTEMATIC MEASUREMENT DIFFERENCES IN THE INTRAOBSERVER REPETITIONS 


\section{LIST OF TABLES}

Table 4-1. Two-way factorial ANOVA models testing for comparability of the malocclusions at the initial records examinations. ........................................35

Table 4-2. Listing of the Dahlberg statistic for the 112 variables when remeasured.. ...38

Table 4-3. Statistics of the eight statistically significant (alpha $=0.05$ ) variables when testing for systematic differences between intraobserver repeatability sessions.

Table 4-4. List of the statistically significant treatment differences abstracted from

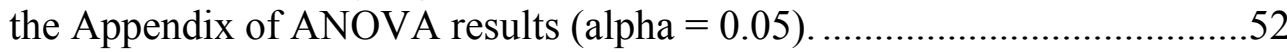




\section{LIST OF FIGURES}

Figure 2-1. Schematized frontal view and description of MARA...................................

Figure 3-1. Measure of condylar distance from the midsagittal plane and the measure of the anteroposterior bilateral asymmetry present

Figure 3-2. Condylar angulation, anteroposterior width of condyles, and mediolateral width of condyles.

Figure 3-3. Joint spaces between condyle and glenoid fossa. ........................................20

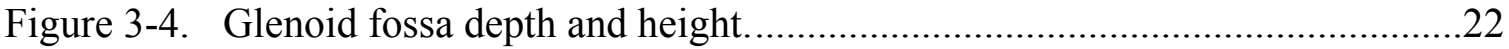

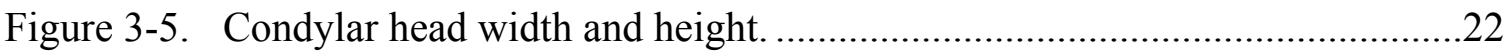

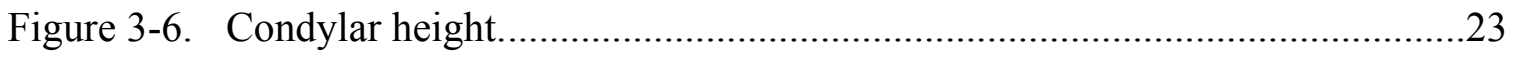

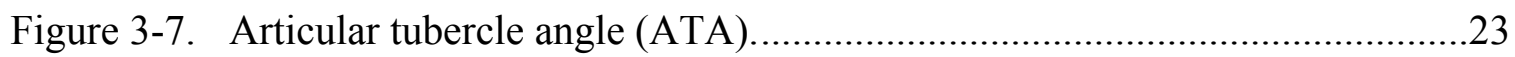

Figure 3-8. Showing the measurements of area of condylar process (AC), area of articular eminence (AAE) and area of glenoid fossa (AGF)........................24

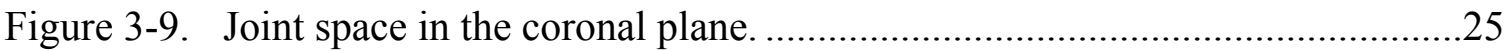

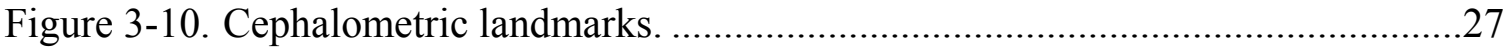

Figure 3-11. Illustration showing incisor overjet and overbite.........................................28

Figure 3-12. Illustration of the cephalometric measurement Pterygoid-Vertical to $\mathrm{M}$ point, where $\mathrm{M}$ is the distal-most aspect of the mandibular symphysis.......29

Figure 3-13. Illustration of the cephalometric measurement SNA....................................31

Figure 3-14. Illustration of the cephalometric measurement SNB ....................................32

Figure 4-1. A metaphor of a 'bull's eye' characterizes the concepts of precision and

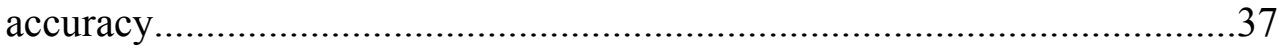

Figure 4-2. Bland-Altman plot for the angle ANB at the initial examination.................42

Figure 4-3. Bland-Altman plot for MLCP Right at the final examination......................43

Figure 4-4. Bland-Altman plot for Condyle LJS Left at the final examination. ..............44

Figure 4-5. Bland-Altman plot for Mesial Molar Relationship at the initial examination. 
Figure 4-6. Bland-Altman plot for Overbite at the final examination.........................46

Figure 4-7. Bland-Altman plot for AJS Left at the final examination...........................47

Figure 4-8. Bland-Altman plot for Sagittal EAM-P Right at the initial examination. ....48

Figure 4-9. Bland-Altman plot for Sagittal FW Left at the initial examination.............49

Figure 4-10. Box plots of the in-treatment changes in the angle SNA...........................53

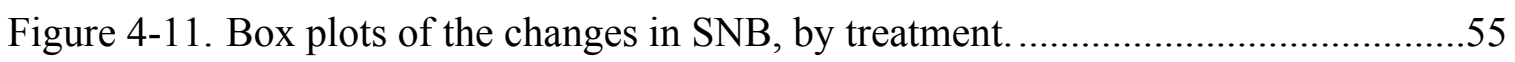

Figure 4-12. Box plots of the in-treatment changes in Axial AP, by treatment. ...............56

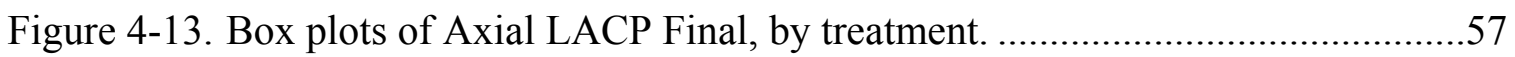

Figure 4-14. Box plots of the in-treatment change in Axial LACP, by treatment.............58

Figure 4-15. Box plots of the Sagittal MJS dimension at the start of treatment...............59

Figure 4-16. Box plots of the in-treatment changes in the Sagittal MJS dimension, by

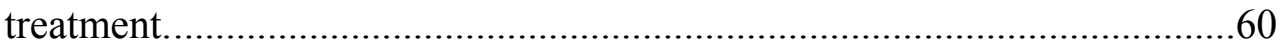

Figure 4-17. Box plots of the Sagittal FD dimension, by treatment...............................61

Figure 4-18. Box plots of the Sagittal CH dimension, by treatment. ............................62

Figure 4-19. Box plots of the Sagittal in-treatment change in the $\mathrm{CH}$ dimension, by treatment.

Figure 4-20. Box plots of the Sagittal EAM-AE dimension at the start of treatment. .....64

Figure 4-21. Box plots of the Sagittal EAM-AE dimension at the end of treatment. .......65

Figure 4-22. Box plots of the Sagittal ATA at the start of treatment. ............................67

Figure 4-23. Box plots of the Sagittal ATA at the end of treatment. .............................68

Figure 4-24. Box plots of the in-treatment changes in Sagittal ATA............................69

Figure 4-25. Box plots in Sagittal ACP at the start of treatment.................................70

Figure 4-26. Box plots in Sagittal ACP at the end of treatment..................................71

Figure 4-27. Box plots in Sagittal AGF at the start of treatment..................................72

Figure 4-28. Box plots of the in-treatment changes in Sagittal AGF............................73 


\section{LIST OF ABBREVIATIONS}

$\begin{array}{ll}\text { AAE } & \text { Area of articular eminence } \\ \text { ACP } & \text { Area of condylar head } \\ \text { AE } & \text { Articular eminence } \\ \text { AGF } & \text { Area of glenoid fossa } \\ \text { AJS } & \text { Anterior joint space } \\ \text { AP } & \text { Anteroposterior condylar length } \\ \text { APCD } & \text { Anteroposterior condylar difference } \\ \text { ATA } & \text { Articular tubercle angle } \\ \text { Ax } & \text { Axial plane } \\ \text { CA } & \text { Condylar angulation } \\ \text { CBCT } & \text { Cone beam computed tomography } \\ \text { Cd } & \text { Condylion } \\ \text { CH } & \text { Condylar process height } \\ \text { CHA } & \text { Condylar head angle } \\ \text { CHH } & \text { Condylar head height } \\ \text { CHW } & \text { Condylar head width } \\ \text { Co } & \text { Coronal plane } \\ \text { EAM } & \text { External auditory meatus } \\ \text { EAM-AE } & \text { External auditory meatus to articular eminence } \\ \text { EAM-C } & \text { External auditory meatus to center of condyle } \\ \text { EAM-P } & \text { External auditory meatus to posterior wall of glenoid fossa } \\ \text { F } & \text { Final } \\ \text { FD } & \text { Fossa depth } \\ \text { FW } & \text { Fossa width } \\ \text { I } & \text { Initial } \\ \text { Inc } & \text { Sigmoid notch } \\ \text { KS } & \text { Kansas } \\ \text { L } & \text { Left } \\ \text { LACP } & \text { Largest area of condylar process } \\ \text { LJS } & \text { Lateral joint space } \\ \text { M } & \text { M point } \\ \text { MARA } & \text { Mandibular Anterior Repositioning Appliance } \\ \text { MJS } & \text { Medial joint space (Coronal) } \\ \text { MJS } & \text { Middle joint space (Sagittal) } \\ \text { ML } & \text { Mediolateral condylar length } \\ \text { MLCP } & \text { Mediolateral condylar position } \\ \text { MSP } & \text { Midsagittal plane } \\ \text { OB } & \text { Overbite } \\ \text { OJ } & \text { Overjet } \\ \text { PJS } & \text { Posterior joint space } \\ \text { R } & \text { Right } \\ \text { Sa } & \text { Sagittal plane } \\ \text { Sella-V } & \end{array}$


Sella-V-to-M

SF

SJS

TMJ
Anteroposterior distance from Sella- $\mathrm{V}$ to $\mathrm{M}$ point Superior aspect of glenoid fossa

Superior joint space

Temporomandibular joint 


\section{CHAPTER 1. INTRODUCTION}

Class II skeletal malocclusion is a common orthodontic problem, with one third of adolescents having this relationship (Cozza et al. 2006). This skeletal disharmony can be due to maxillary jaw prognathism or mandibular retrognathism, though the more common feature in this malocclusion is insufficient mandibular length (Graber et al. 2005). Several forms of treatment can be used to correct this discrepancy. Traditional forms include extra-oral appliances such as headgear, surgical procedures such as tooth extractions and orthognathic surgery, intraoral elastics, and functional orthopedic appliances. Each of these treatment modalities "differ in their effect on the skeletal structures of the craniofacial region, sometimes accelerating or limiting the growth of the various structures involved" (McNamara and Brudon 1993). Functional appliances may provide treatment for the growing patient with retrognathic profile features (Allen-Noble 2005).

The term "functional appliance" refers to the variety of removable and fixed appliances designed to alter the development of muscle groups that influence the function and position of the mandible. This is usually obtained by altering the mandible sagittally and vertically with the intent of creating orthodontic and orthopedic changes (Bishara 1989).

Functional appliances have been designed to position the mandible forward with the intent of enhancing mandibular growth. The mandible is directed into a protruded position and held there. Consequently, the condyle, also, is displaced anteriorly in its glenoid fossa. Proffit et al. state that, when using a functional appliance, "lengthening of the mandible has much less than the expected effect on Class II skeletal malocclusion because the temporomandibular joint (TMJ) remodels posteriorly at the same time the mandible is growing longer" (2007). Studies have shown that functional appliances have effectively enhanced growth of the mandible in skeletal Class II patients. Published research in humans has reported anterior and inferior relocation of the glenoid fossa, minor increased mandibular length, and posterior redirection of condylar growth associated with fixed functional appliance treatment (Popowich et al. 2003). Histological studies using laboratory animals have repeated shown an increase in cellular activity in the TMJ due to fixed functional appliance therapy (Woodside et al. 1987; Charlier et al. 1969).

Other investigators have discounted these statements, believing that redirection of mandibular growth does not occur in humans with functional appliance therapy. Some studies have suggested that the changes might be only those expected with normal growth or conventional edgewise therapy (Creekmore et al. 1983; Gianelly et al. 1983). Several researchers have considered that the Class II correction observed with functional appliances was caused by a "headgear" effect restraining maxillary growth along with a combination of dentoalveolar changes such as retroclination of the maxillary incisors and proclination of the mandibular incisors (Creekmore et al. 1983; Hotz 1970). 
Several studies have analyzed condyle-fossa modification in human subjects with fixed functional appliance treatment. Most of the studies have used lateral cephalograms or computed tomography to measure mandibular growth in fixed functional appliance cases (Woodside et al. 1987; Voudouris et al. 2003; VanLaeken et al. 2006). These forms of radiography, however, have been shown to be inaccurate. Lateral cephalograms have been shown to vary from real values of objects over half the time (Hilgers et al. 2005), and computed tomography has been shown to be less diagnostic than cone beam computed tomography (CBCT) (Honey et al. 2007). No study has used CBCT images to measure TMJ changes in humans receiving fixed functional appliance treatment.

A fixed functional appliance used in clinical orthodontics is the mandibular anterior repositioning appliance (MARA). This appliance has increased in popularity over the last decade due to having similar results to the Herbst appliance while having less breakage and cheek irritation (Allen-Noble 2005). Due to its recent emergence in clinical orthodontics, few studies have evaluated the MARA.

The purpose of the present study was to obtain measurements of the condyle relative to the glenoid fossa in patients treated with the MARA. Pre- and post-treatment $\mathrm{CBCT}$ images were measured, and these changes were compared with those obtained from conventional Edgewise orthodontic treatment with Class II intermaxillary elastics. 


\section{CHAPTER 2. LITERATURE REVIEW}

\section{Structure of the Temporomandibular Joint}

\section{Introduction to the Joint}

The articulation of the mandible with the cranium is unlike any other joint in the body. The mandible effectively bridges a bilateral articulation composed of left and right temporomandibular joints that form a functioning unit. Each joint influences the other, and each joint cannot function separately.

Each temporomandibular joint is a diarthroidial joint which permits more freedom of movement between the parts than in a fibrous joint (i.e., suture) or gomphosis (i.e., tooth in socket). The articular surfaces of the TMJs are made of tissues that are capable of bearing a compressive load and movement simultaneously. Because of the need to withstand compressive forces, the articular tissues are without blood vessels and nerve receptors in load bearing areas. The inner lining of the capsule that surrounds the articular tissues secretes synovial fluid to supply the nutrient needs to the nonvascularized articular tissues.

Each TMJ is compound, involving more than two bones. In the TMJ, the articular disc serves as the "third bone" (Bell 1982). In essence, each joint is a double joint composed of a lower ginglymoid joint and an upper arthroidial joint. The TMJ has been described as a hinge joint with a moveable socket (Sicher 1949). It is ginglymoid because the disc-condyle complex is a hinge joint, where the condyle articulates with the disc. It is arthroidial due to the disc-condyle complex sliding down the temporal bone. The lower joint is a hinge, while the upper joint is designed for sliding movement in any direction. The TMJ articulation is the only bilateral ginglymo-arthroidial joint in the human body.

\section{The Condyle}

The condyle is the portion of the mandible that articulated with the cranium, around which movement occurs (Okeson 2003). Although a considerable amount of variation exists in its morphology, the articular surface of the mandibular condyle is generally rounded and its long axis is oriented in a mediolateral direction (Graber 2005). The angle of this axis with the frontal plane varies from zero to thirty degrees (Öberg and Carlsson 1979). The articular surface of the condyle is quite convex anteroposteriorly and only slightly convex mediolaterally. The total mediolateral width of the condyle averages about 15 to $20 \mathrm{~mm}$, and the anteroposterior width average is around 8 to $10 \mathrm{~mm}$ (Okeson 2003). The condyle's posterior margin extends farther than that of the anterior, which allows for greater posterior rotation of the articular disc (Bell 1982). The anterior and posterior portions of the articular surface slope down from a transverse ridge at the 
most superior point of the condyle. From the anterior view, the condyle has a medial and lateral projection called poles. The medial pole is generally more prominent than the lateral pole. These poles are just below the articular surface and are marked by bony tubercles for the attachment of the capsule and disc (Okeson 2003).

Unlike articular surfaces in synovial joints, the condyle's articular surface is not composed of hyaline cartilage; instead, it consists of a dense fibrous connective tissue that is nonvascular and noninnervated. The tissue is quite thin over most of the articular surface, but thickens appreciably in the anterosuperior portion, indicating the area best suited to sustain biomechanical pressure (Öberg and Carlsson 1979; Bell 1982). In contrast with the articular area of growth plate cartilage, the cells of this fibrous connective tissue so not take part in condylar growth and remodeling, but only proliferate as needed to maintain the articular surface (Degroote 1984).

Petrovic et al. point out some additional differences between typical cartilage seen in long bones and the mandibular condyle (1975). The chondroblasts of primary cartilage is usually lined up in rows of cells, while the arrangement of condylar cartilage is much more erratic. Also, the prechondroblastic cells are dividing and proliferating in the condylar cartilage, while differentiated chondroblasts do the same in epiphyseal plates (Petrovic et al. 1975). The cells diving in the condylar cartilage are those that are not yet synthesizing cartilaginous matrix.

Immediately below the dense collagenous articulating surface of the condyle is a region of undifferentiated mesenchyme from which the preosteoblasts and prechondroblasts proliferate (Petrovic et al. 1975; Öberg and Carlsson 1979; Bell 1982). These cells mature into osteoblasts in areas of slow growth, or into chondroblasts in areas of rapid growth (Öberg and Carlsson 1979). The chondroblasts quickly lose their ability to divide and hypertrophy, leaving a limited deposition of intracellular matrix in the deep part of the proliferative zone or zone of maturation (Petrovic et al. 1975). The matrix surrounding the cells in the lower part of the zone of maturation become calcified in a pericellular way (Degroote 1984). This pattern is in contrast to that in epiphyseal cartilage where calcification takes place along vertical lines of cartilaginous matrix.

In the zone of erosion, the chondroblasts degenerate and chondroclasts resorb the cartilaginous matrix that is replaced by endochondral bone. Resorption of this mineralized cartilage is mediated by chondroclasts which are closely associated with the terminal ends of the penetrating blood vessels (Durkin 1972). This is in contrast to the process in primary cartilage when capillaries enter to non-calcified portions of the cartilage while chondroclasts are resorbing the mineralized cartilaginous septa (Degroote 1984). The endochondral formation of bony trebeculae underneath the zone of erosion in the condyle also differs for primary cartilage where bone trebeculae are formed using the calcified cartilage as a matrix (Durkin et al. 1973). 


\section{Remodeling and Osseous Change}

The tissues of the condyle serve the function of true articular cartilage due not only to their ability to withstand movement and a compressive load, but also their capability for regeneration and remodeling (Bell 1982). Structural adaptation of the articular surfaces of the temporomandibular joint is necessary for normal growth and development of the face as well as changing demands in function in the masticatory system throughout life (Öberg and Carlsson 1979; Bell 1982).

Remodeling in the TMJ may be classified as either progressive or regressive. Progressive remodeling involves the proliferation of tissue, while regressive remodeling is characterized by bony resorption. Remodeling is reflected by an increase in the activity of the cells of the proliferative zone, but the composition and thickness of the different cell layers varies in adults owing to the character and location of the remodeling processes. A cartilage layer exists throughout life superiorly and anteriorly on the condyle and posteroinferiorly on the articular eminence of the temporal bone due to the continued functional load (Öberg and Carlsson 1979). Studies have shown that natural changes occur more in the condylar surface than the temporal surface, while little or no remodeling occurs in the articular disc (Hansson et al. 1977).

The amount of compressive force acting on the tissue of the tissues of the TMJ is important in influencing the remodeling changes occurring within stress-bearing areas. Moderate loading facilitates normal remodeling, while excessive loading arrests remodeling and may induce metaplasia of the cartilage not only in the articular surface of the condyle but also the temporal surface as well. If compressive stress is even greater, localized resorption can occur (Bell 1982).

All remodeling changes are time dependent (Bell 1982). Time permitting, adaptive changes of the TMJ can occur, but if the demands for change are too rapid, degenerative remodeling may take place instead. Age is a factor related to this process. Adaptive processes are seemingly quite adequate during the developmental period and remain so well into adult life, but in later years the decrease in response suggests a serious decline in adaptive and regenerative capacity (Öberg and Carlsson 1979; Bell 1982). Tissues in older adults require more time than those of younger individuals to affect the same amount of change in the adaptive response.

\section{Functional Appliances}

\section{History}

Pierre Robin designed the earliest removable functional appliance in the early 20th century. Coined the "monoblock", it was not intended for mandibular repositioning; its use was for passive positioning of the tongue in neonates with mandibular retrognathism and cleft lip and palate. Vigo Andresen, unaware of the Robin appliance, 
created the activator in the 1920s, a passive tooth-borne appliance. He added a horseshoe flange to a Hawley retainer that guided the mandible three to four millimeters forward; this created a sagittal correction primarily in Class II, division I malocclusions (Graber et al. 2005). The functional regulator, designed by Fränkel in 1967, was the first tissueborne removable appliance that stimulated mandibular repositioning. Clark designed the twin-block appliance in 1977, which consisted of maxillary and mandibular plates that guide the mandible forward when the patient closes (Proffit et al. 2007).

Emile Herbst introduced the Herbst appliance in the early 1900s. This was the first fixed functional appliance that was used as a bite-jumping device for Class II treatment. Pancherz reintroduced this appliance in the 1970s (VanLaecken et al. 2006). The appliance was designed to splint the maxilla and mandible with bonded bands connected by a pin and tube device that held the mandible forward. The appliance was modified to replace the bands with crowns in order to avoid breakage of the appliance (Proffit et al. 2007). The appliance treatment time was relatively short compared to previous removable functional appliances and required less patient cooperation since the appliance was fixed in the mouth.

\section{Mandibular Anterior Repositioning Appliance}

Douglas Toll of Germany designed the mandibular anterior repositioning appliance, a fixed functional appliance, in 1991. Jim Eckhart, a dentist in California, had been using functional appliances in the 1970s and 80s and sought an appliance that would minimize breakage and misplacement. He used the Herbst fixed functional appliance with some success, but patients were complaining of lip and cheek irritation. Eckhart and Toll experimented with improving the design (Allen-Noble 2005).

The MARA allegedly produces similar effects to the Herbst appliance: mandibular teeth move anterior, maxillary growth is restrained, the glenoid fossa is remodeled, and condylar growth is stimulated (Allen-Noble 2005). A basic MARA consists of the following parts (Figure 2-1):

1. Four crowns (or bands) on the first permanent molars,

2. Lower arms soldered to the lower molar crowns to help with positioning of the mandible and buccal cheek comfort,

3. Soldered maxillary and mandibular archwire tubes,

4. Maxillary elbow tubes for placement of MARA elbows,

5. Elbows that are placed with shims in the tubes to provide the amount of desired advancement, and 


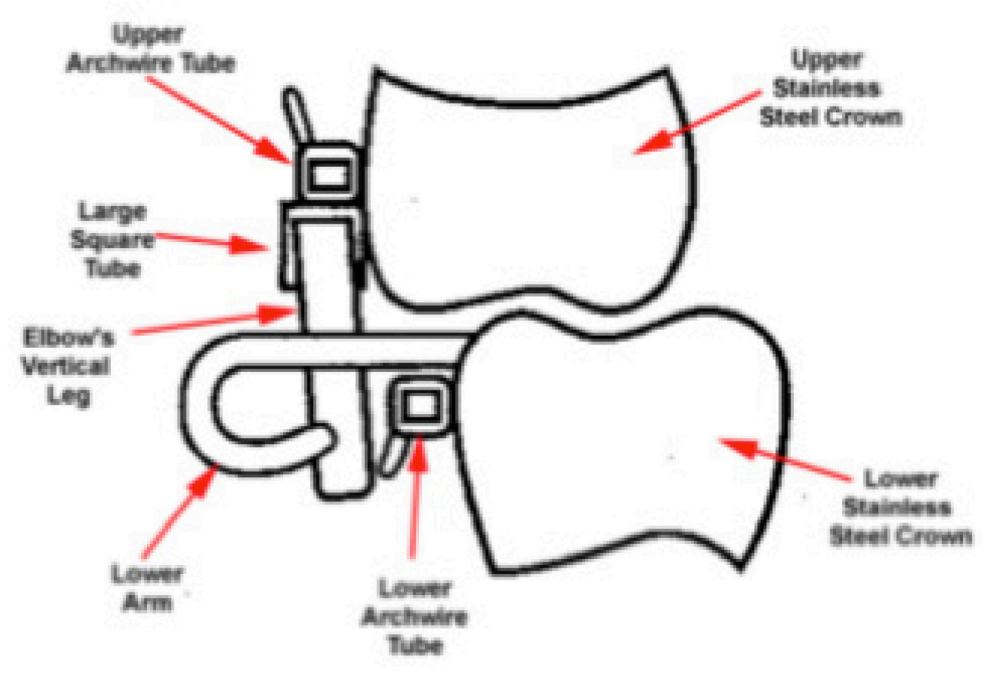

Figure 2-1. Schematized frontal view and description of MARA.

6. Lower lingual arch attached to lower molars, which is recommended in order to keep the lower molars from tipping mesially and lingually in response to the forces from the elbow.

This design can also be modified to include maxillary and/or mandibular expansion, a transpalatal arch, and adjustment loops for the lower lingual arch (Noble-Allen 2005).

The MARA has been used for various reasons to aid in orthodontic treatment. It is mainly considered for the advancement of the lower jaw in growing children when the position of the upper jaw is acceptable. The MARA also has a reported headgear effect, although it is less noticeable than with use of the Herbst appliance (Pangrazio-Kulbersh et al. 2003). The MARA can also be used on adult patients as an alternative if the patient declines orthognathic surgery; however, the change is more dental than skeletal (AllenNoble 2005).

The MARA has some advantages over traditional functional appliances. Upon introduction of the appliance in the mouth, the profile immediately looks improved. Class II malocclusion are simply treated by inserting different length shims on either elbow, which also help in correcting Class II asymmetries. The MARA also gives a minimal distal movement and the desired mesiobuccal rotation to the maxillary first molars. In comparison to removable appliances, patient cooperation and treatment times are not an issue with the MARA because the appliance is fixed. The MARA also has few breakage concerns, and speech and hygiene are not a problem (Allen-Noble 2005).

In a study by Pangrazio-Kulbersh et al. (2003), the MARA was compared to the Herbst and Fränkel II appliances and to an untreated group. The authors used lateral cephalograms at pre- and posttreatment to measure the amount of dental and skeletal changes. The MARA provides more dentoalveolar changes than the Fränkel II appliance 
while producing similar effects to the Herbst appliance. They did not study TMJ changes due to functional appliance treatment.

\section{Temporomandibular Changes with Functional Appliances}

Several studies have measured the amount of change in the temporomandibular joint under various conditions. These studies involving functional appliances have been initiated to determine the overall efficacy of functional appliances in correction of Class II dental and skeletal malocclusion, and they measure the TMJ to determine if there is any remodeling present.

\section{Proponents of Condylar Remodeling and Modified Growth}

In a study of primates, Woodside et al. (1987) examined the changes in the condyle and glenoid fossa following a period of gradually activated and constantly maintained advancement of the mandible using the Herbst appliance. The study consisted of six female and one male cynomolgus monkeys, comprised of five adolescents, one juvenile, and one adult subject. The advancement was achieved by adding stops to the telescopic arms of the appliance, with the total activation reaching 7.0 to $10.0 \mathrm{~mm}$, dependent upon the span of the treatment. They measured patient lateral cephalograms pre- and posttreatment and observed decalcified histologic segments of the fossa and condyle in order to determine remodeling of the TMJ. The mandibular advancement was found to produce extensive remodeling and anterior relocation of the glenoid fossa, which contributed to anterior mandibular positioning and altered jaw relationships. The authors state that this remodeling of the glenoid fossa "may create the appearance of an increased mandibular length with or without a true increase"(p. 196).

In a well-documented study by McNamara et al. (1987), twenty-three mate juvenile rhesus monkeys (Macaca mulatta) were used in an experimental study of longterm mandibular adaptations to induced protrusive function. Successive protrusive appliances were placed in 11 experimental animals and mandibular adaptations were monitored cephalometrically. Twelve animals were used as controls. After 48 weeks significant increases in increments of condylar growth and in overall mandibular length were noted in the treated animals. At the end of the 144-week experimental period, the mandibles of the treated animals were 5 to $6 \mathrm{~mm}$ longer than those of the control animals. Their results do not support the hypothesis that the mandible has a genetically predetermined length (McNamara et al. 1987).

Voudouris et al. (2003) also used the Herbst appliance in growing nonhuman primates, and the results were compared with primate and human controls. They used cephalometric analysis plus intravenous tetracycline vital staining, histological assessment, and electromyographic analysis to study the response of the glenoid fossa associated with continuous mandibular protrusion. All experimental primate subjects developed large super Class I malocclusions, the result of many factors including: 
headgear effect on the maxillary arch and teeth, an improved horizontal component of condylar growth, and anterior displacement of the mandible and mandibular teeth. The growth modification measured in the glenoid fossa was in an inferior and anterior direction. The authors proposed that constraint of the downward and backward growth of the fossa, which was observed in the control subjects, might additionally contribute to the overall super Class I malocclusion. The authors state that these combined effects could be significant at the fossa; differences in the area and maximum thickness of new bone formation in the glenoid fossa and in condylar growth were statistically significant in experimental subjects. These bony changes in the condyle and the glenoid fossa were correlated with decreased postural electromyographic activity during the experimental period. Results from permanently implanted electromyographic sensors in the primates established that lateral pterygoid muscle hyperactivity was not linked with condyle and glenoid fossa growth modification with functional appliances, and that stretch forces along the fibrocartilage between the displaced condyle and fossa might play a more significant role in new bone formation. This study reaffirmed the growth relativity theory, which states that the stretched soft tissues surrounding the condyle and the glenoid fossa contribute to bone growth (Voudouris et al. 2000).

Ruf et al. (1999) analyzed and compared the TMJ adaptive mechanisms in 25 adolescent and 14 young adults with Class II malocclusions treated with the Herbst appliance. TMJ remodeling was analyzed by magnetic resonance imaging (MRI). In each subject, 4 magnetic resonance images of both temporomandibular joints were available: before treatment, at the initiation of treatment (when the Herbst appliance was placed), during treatment ( 6 to 12 weeks after appliance placement), and after treatment (when the appliance was removed). In addition, effective temporomandibular joint changes (the sum of condylar remodeling, fossa remodeling, and condyle-fossa relationship changes) were analyzed with the aid of lateral cephalometric radiographs from before and after treatment. All subjects were treated to a Class I (or overcorrected Class I) dental arch relationship, and their mandibles became significantly more prognathic. After 6 to 12 weeks of Herbst treatment, signs of condylar remodeling were seen at the posterosuperior border in 48 of the 50 adolescent condyles and in 26 of the 28 young adult condyles. Indications of glenoid fossa remodeling at the anterior surface of the postglenoid spine were noted in 36 adolescent and 22 young adult temporomandibular joints. Bilateral remodeling of the mandibular ramus could be detected in 1 adolescent and 2 young adult patients. TMJ changes during treatment were more horizontally directed and larger in both adolescents and young adult patients treated with the Herbst appliance than in an untreated group of subjects with ideal occlusion. The authors believe that mandibular retrognathism correction achieved by Herbst therapy in both adolescents and young adults seemed, in particular, to be a result of condylar and glenoid fossa remodeling. MRI rendered better visualization of the temporomandibular joint remodeling growth processes than traditional radiography such as lateral cephalograms (Ruf et al. 1999).

VanLaecken et al. (2006) studied 32 subjects (18 females and 14 males) treated with the edgewise Herbst appliance by one of the authors. The position of the condyle relative to the glenoid fossa was determined by using horizontally corrected axis tomograms taken pretreatment and posttreatment. At the end of Herbst treatment, the 
tomograms showed only $0.2 \mathrm{~mm}$ of forward movement of the condyle within the fossa with no significant change in joint space. The minimal change in the condylar position relative to the fossa suggests a combination of fossa remodeling and redirection of condylar growth after Herbst treatment (VanLaecken et al. 2006).

Rabie et al. (2002) designed a study to identify the relationship between vascularization and bone formation in the glenoid fossa during natural growth and functional appliance therapy. The sequential pattern of vascular endothelial growth factor (VEGF) expression and bone formation in the glenoid fossa during normal growth was identified and compared with that during forward mandibular positioning with a functional appliance. A close correlation has been found between vascularization and bone formation (Rabie et al. 2002). They used 150 female Sprague-Dawley rats averaging 35 days old, and split the subjects into 10 experimental and 10 control groups. Appliances were fitted to position the mandible forward in the experimental groups. The rats were then sectioned and stained with anti-VEGF antibodies to evaluate VEGF expression, and new bone formation was evaluated using Schiff's reagent. The results demonstrated that, during normal growth and forward mandibular positioning, VEGF expression and new bone formation were highest in the posterior region of the glenoid fossa, and significant increases of VEGF and new bone formation were seen in the experimental groups compared with the controls. The highest amount of VEGF expression occurred before the highest amount of bone formation was reached. The authors concluded that forward mandibular positioning cause significant increases in vascularization and new bone formation in the glenoid fossa (Rabie et al. 2002).

In a classic study by Pancherz and Ruf (1998), the authors intended to discover tempormandibular growth adaptation due to Herbst treatment by evaluating condylar remodeling, glenoid fossa remodeling, and condyle-fossa relationship changes. The sample included consecutively treated patients with Class II malocclusion and full permanent dentition. Based on hand-wrist radiographs to determine skeletal maturity, the sample was subdivided into adolescents (12 girls, 13 boys) with a mean age of 12.8 years (range, 11.4-15.7) and young adults (19 girls, 4 boys) with a mean age of 16.5 years (range, 13.6-19.8). Parasagittal MRIs were obtained pretreatment, shortly after appliance placement, 6 to 12 weeks after appliance placement, and shortly after appliance removal. They reported "signs of remodeling" described as bone apposition, being the greatest at the inferior part of the glenoid spine. They also noted a slight flattening of the spine. In terms of condyle remodeling, they found that the young adult group had a "double contour" in the superior posterior region of the condylar head, while the adolescents had no change. They also found that the condylar position in the fossa remained unaffected on average.

\section{Opponents of Functional Appliance Therapy}

Proponents of functional appliance therapy claim that propulsion on the mandible forward will cause a shift in condylar growth potential that would have not been achieved with conventional, full-banded orthodontic treatment. The following studies argue that 
the mandible has a genetically predetermined length and that conventional orthodontic therapy can correct the Class II skeletal patient.

According to Lysle Johnston (1998), the amount an individual can be anticipated to grow during treatment might or might not depend on the particular mode of treatment, yet growth is partly a function of age, sex, and time of treatment. Growth curves from any of the large growth studies (Burlington, Bolton, or Michigan) can provide good estimates of growth intensity. Johnston (1998) studied facial growth from lateral cephalograms of 120 Class II, division 1 patients treated between 1969 and 1980 at St. Louis University as part of a long term comparison between non-extraction and extraction edgewise therapy. Only 12 of the 120 patients studied showed the mandible failing to advance relative to the maxilla during treatment. Six of these 12 were treated by extraction therapy and six were treated by non-extraction therapy. This and thirty years of clinical experience led Johnston to conclude that most Class II patients show a favorable growth pattern during conventional orthodontic treatment (Johnston 1998).

Hotz (1970) described cases that he had treated using a removable functional appliance that was similar to the Andresen activator. These cases were Class II, division I patients that had been treated using the functional appliance, a lower lingual arch, and intermaxillary Class II elastics. While some patients responded with mandibular growth, he concluded that most of the correction was due to dental correction with incisor tipping (Hotz 1970).

Creekmore (1983) compared pre- and posttreatment lateral cephalograms of 20 growing children who had either Class I or Class II Fränkel functional regulator therapy to 50 patients who had undergone conventional orthodontic banding with headgear and an untreated control sample. All of the treated subjects had full Class I correction from whichever mode of therapy was given. He found that there was no significant difference in the forward growth of the mandible in the Class I Frankel, Class II Frankel, and untreated samples. In the edgewise-treated sample the mandible came forward $1 \mathrm{~mm}$ less than in the other samples and was statistically significant. The Class II Frankel sample demonstrated a significant reduction in the ANB angle as compared to the untreated sample, and this reduction was significantly less than the reduction that occurred from edgewise treatment. He also noted that the increased protraction of the mandible in the Class II Fränkel sample, compared to the Class I sample, made no significant difference in yearly growth increments for mandibular length, maxillary length, maxillomandibular differential, and lower face height. The horizontal component of growth or movement of the condyle was significantly greater for the Fränkel samples as compared to the edgewise sample, which, in turn, was significantly greater than the untreated sample. The significance of the increased horizontal component is that the direction of condylar growth is more backward for the treated samples. Condylar growth for the Class II Fränkel sample was $8.5 \mathrm{~mm}$ at 35 degrees from the vertical, whereas for the untreated control it was $7.4 \mathrm{~mm}$ at 13 degrees. However, he argues that if the correction was completely orthopedic, then the amount of condylar growth should have averaged about $13 \mathrm{~mm}$ rather than $8.5 \mathrm{~mm}$. He concluded that many of the skeletal changes attributed to treatment occur in untreated persons, and many of the changes are found with equal 
facility in the edgewise-treated cases (Creekmore 1983).

Gianelly et al. (1983) also compared functional appliance wear to conventional banded orthodontic therapy. They evaluated laminographs pre- and posttreatment of 10 Class II, division 1 patients between the ages of 9 and 13 that wore Fränkel functional regulators for one year. They compared this sample to a sample of 15 Class II, division 1 growing patients that underwent fixed orthodontics with cervical traction. They found a wide range of mandibular growth in both groups, but the average growth for both groups was the same $(2.4 \mathrm{~mm})$. SNB also did not change significantly before and after treatment for the Fränkel group. They also measured the amount of space between the most superior point of the condyle and fossa before and after treatment, and they used a leaf gauge to determine whether the condyle was fully seated in the fossa. They found that 6 of the 10 Fränkel subjects did not present with the condyle fully seated in the fossa after appliance therapy, and the average supracondylar space increased by $1.0 \mathrm{~mm}$. Therefore, the authors argue that fixed banded appliances show the same amount of mandibular growth with no increases in condylar space, unlike removable functional appliance wear (Gianelly et al. 1983).

\section{Cone Beam Computed Tomography}

Modern updates in cone beam computed tomography (CBCT) show potential for future developments in craniofacial imaging. With radiation doses being lower than those of classical computed tomography (CT) imaging, they allow the clinician a viable option of viewing craniofacial structures 3 dimensions. There are several applications in the field of orthodontics (Baumrind 2011).

Like any diagnostic imaging field, there are both advantages and disadvantages of $\mathrm{CBCT}$. The tremendous advantage is that it greatly increases the information about the craniofacial morphology of individual patients that is available to clinicians and clinical investigators. Once the volumetric dataset has been constructed, it can be sliced in any number of desired planes to produce projection images of all the types with which we are already familiar. These include pseudo lateral and frontal cephalograms, panoramic images, or individual periapical images. Moreover, it can also produce types of images that were never possible before, including thin slices through the volume without confounding overlap from structures in front of or behind the slice. Images of this sort can be oriented in any desired plane and are especially valuable for the analysis of temporomandibular joint disorders. The system can also produce projection images of regions of the head with other confounding structures digitally dissected away-for example the condyle separated from the glenoid fossa. Planar images viewed from different perspective — sagittal, para-sagittal, coronal, or oblique — can be generated as desired. It is also possible to produce lateral cephalograms that remove material from one side of the midsagittal plane, allowing the buccal segment dentition on the remaining side to be viewed without overlap from the other side. In addition, the future of this technology is bright, as many new ways of slicing the volumetric dataset will be 
developed by innovative clinicians, intended to meet the needs of the clinical practice (Baumrind 2011).

\section{Efficacy of CBCT in Measuring the TMJ}

The most commonly used radiographs to assess the temporomandibular joint are panoramic radiographs, transcranial projections, and tomography (Honey et al. 2007). Several authors have reported high accuracy in using CBCT to evaluate the TMJ region.

Honey et al. (2007) conducted a cross-sectional in-vitro study to compare the diagnostic accuracy of observers viewing images made with CBCT, panoramic radiography, and linear tomography. They measured this by detecting cortical erosions affecting the mandibular condylar head. The sample consisted of 37 TMJ articulations from 30 skulls with either normal condylar morphology or erosion of the lateral pole. They used corrected linear tomography (TOMO), normal and TMJ-specific panoramic radiography, and $\mathrm{CBCT}$ in order to image the TMJ region. Images and ten rereads were presented to ten observers on a flat-panel display. CBCT multi-planar images were presented both statically (CBCT-S) and interactively (CBCT-I). The observers were permitted to scroll through axial $(0.4 \mathrm{~mm})$ and para-sagittal $(1 \mathrm{~mm})$ sections and then independently rate their confidence about the presence or absence of cortical erosion of the TMJ. Pan-N, CBCT-I, and CBCT-S reliability was significantly greater than TOMO. The diagnostic accuracy of CBCT-I and CBCT-S was significantly greater than all other modalities. The authors concluded that CBCT images provide superior reliability and greater accuracy than TOMO and TMJ panoramic projections in the detection of condylar cortical erosion (Honey et al. 2007).

Hilgers et al. (2005) compared TMJ measurements between iCat CBCT and conventional cephalograms. They used true anatomical sites in dried human skull and compared the true measurements to those found using three orthogonal planes - lateral cephalometric, posteroanterior, and submentovertex - in CBCT and conventional radiographic images. They found that all CBCT images were highly accurate, but over half of the conventional films varied significantly from real values. The authors concluded that custom oblique multi-planar reformatted reconstructions with iCAT CBCT provide accurate and reliable linear measurements of mandibular and TMJ dimensions (Hilgers et al. 2005).

\section{Purpose of the Study}

An ongoing debate exists in orthodontics about the amount of condyle-fossa remodeling and growth seen in patients using functional appliance therapy. While some clinicians and researchers have found that that the use of functional appliances increases the amount of condylar growth and subsequent Class II skeletal correction, others believe that the mandible has a preset amount of growth and that conventional orthodontics will result in a similar amount of condylar growth to functional appliance therapy. The 
purpose of this study is to reintroduce this debate by comparing condylar remodeling in functional appliance therapy to conventional fixed orthodontic therapy by measuring changes using $\mathrm{CBCT}$, a more accurate imaging tool than conventional radiography used in the past. We will be looking at changes induced by the MARA, which has not been studied at depth in the condylar region, to conventional full orthodontic treatment using intermaxillary elastics. 


\section{CHAPTER 3. MATERIALS AND METHODS}

\section{Study Design}

The present study was a retrospective analysis of cone beam computed tomography images taken on subjects with Class II malocclusions at the beginning and end of their comprehensive orthodontic treatment. These adolescents received nonextraction treatment with a MARA followed by or coinciding with full fixed Edgewise appliance treatment. Subjects' ages were between nine and fourteen years of age at the initiation of treatment. The study was limited to Class II, division 1 malocclusions by choosing Caucasian subjects with an overjet of at least $3.5 \mathrm{~mm}$ and ANB greater than 4 degrees. Patients must have taken the image in maximum intercuspation, and patients with open bites or posterior crossbites were not used due to functional deviations of the condyles. Any patient with a craniofacial anomaly was also excluded from this study. The CBCT images were available at the beginning and end of treatment with the MARA plus conventional Edgewise orthodontic treatment. Thirty-three subjects' records (13 females, 20 males; average age 11.93 years) were acquired from the office of Dr. James K. Dillehay, Wichita, Kansas, who treated all of these MARA cases (from this point known as Kansas). The conventional Edgewise-only group consisted of a sample of thirty-one subjects (18 females, 13 males; average age 12.37 years) with Class II malocclusions treated non-extraction with full fixed conventional orthodontic appliances from the office of Dr. Daniel R. Merwin, Jackson, Tennessee (from this point known as Tennessee).

The CBCT scans were evaluated using Dolphin 3D (Dolphin Imaging and Management Solutions, Chatsworth, CA). Dolphin 11 is the version that incorporates the 3D module. Each image (pre- and posttreatment) was evaluated at both the right and left TMJ in the axial, sagittal, and frontal planes.

\section{Axial Dimension}

Axial plane reconstruction of the condyles was done in the TMJ module of Dolphin 11. First, the CBCT image was oriented in Frankfort Horizontal using Dolphin 11 software. The TMJ module was entered within Dolphin 11, and the axial representation of the image was shown. The axial slice was evaluated where both the right and left condylar heads were viewed in their greatest area dimension. Then, the images were exported into ImageJ64 (NIH, version 1.44 for the PC) for obtaining measurements. 


\section{Landmarks}

Landmarks used in the axial plane were referenced in Kang et al. (2010) and Rodrigues et al. (2009). The following anatomic landmarks were used for measuring the condyles in the axial plane:

1. Vomer: an unpaired facial bone in the midsagittal plane that rests between the left and right nasal cavities.

2. Clivus: part of the cranium in the midsagittal plane that is a shallow depression sitting just posterior to the sphenoid sinus.

3. Center of the condyle: geometric center of the condylar heads in the axial plane.

\section{Linear Measurements}

Linear measurements of the condyles in the axial plane were measured by exporting the images into Image J64 with a read-out precise to $0.1 \mathrm{~mm}$. These measurements were taken from Rodrigues et al. (2009) in evaluating the TMJ using CBCT images:

1. Mid-Sagittal Plane (MSP): line connecting the vomer and clivus that separates the face into equal left and right sections.

2. Mediolateral width of left $\left(\mathrm{ML}^{1}\right)$ and right $\left(\mathrm{ML}^{\mathrm{r}}\right)$ condyles: greatest length from medial to lateral pole of condyle.

3. Anteroposterior width of left $\left(\mathrm{AP}^{\mathrm{l}}\right)$ and right $\left(\mathrm{AP}^{\mathrm{r}}\right)$ condyles: greatest length anteroposteriorly of condyle in axial plane.

4. Mediolateral condylar position: measurement from the center of the condyle to the mid sagittal plane perpendicularly (Figure 3-1).

5. Anteroposterior condylar difference: difference in right and left condylar position as reflected on the midsagittal plane.

The pre- and posttreatment images were compared. The widths of the condyle were measured to determine any change in the condylar head during treatment. The condyles were referenced to the midsagittal plane to determine any mediolateral positional changes due to functional treatment. The anteroposterior condylar difference was measured from the pretreatment records to determine any jaw asymmetry, and this was compared to the post-treatment image to see if the asymmetry was corrected. 


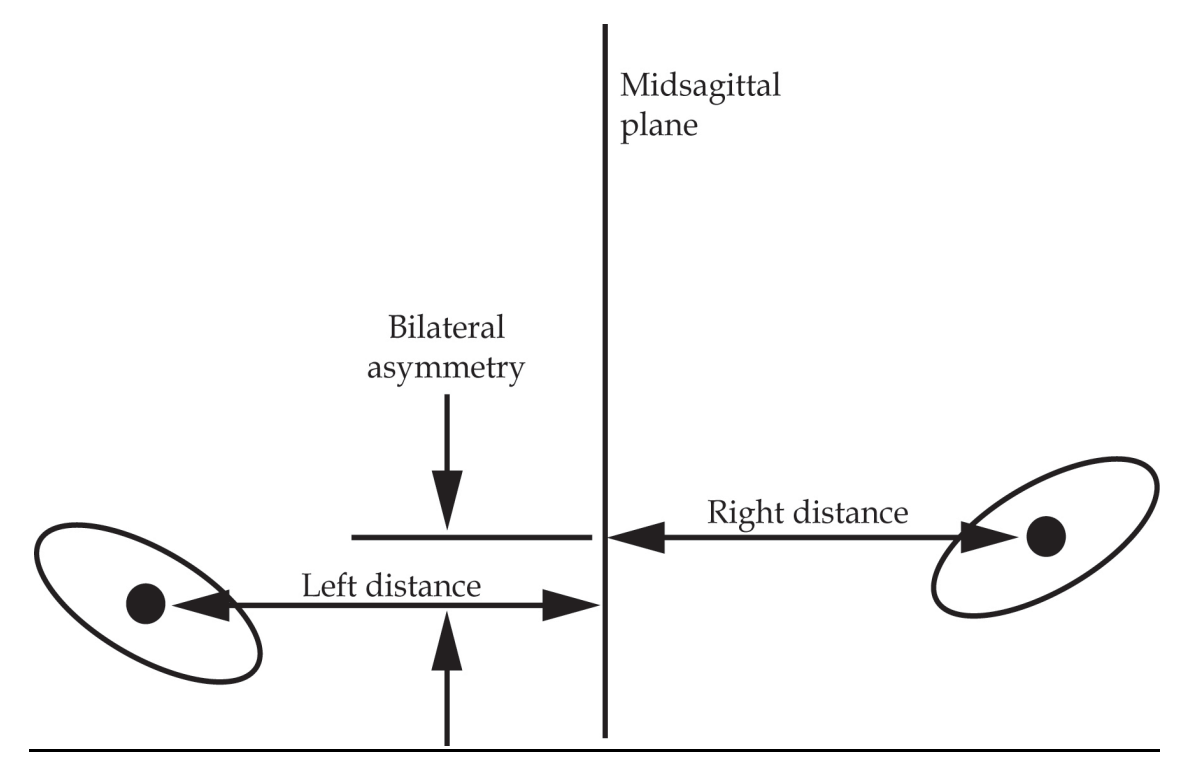

Figure 3-1. Measure of condylar distance from the midsagittal plane and the measure of the anteroposterior bilateral asymmetry present.

\section{Angular Measurements}

Angular measurements of the condyles in the axial plane will be measured by exporting the images into ImageJ64. The measurement used as referenced by Rodrigues et al. (2009) accounts for the angulation of the condylar head (Figure 3-2):

1. Condylar angulation: angle formed by the mid-sagittal plane and the line connecting the medial and lateral poles of the condylar head.

2. The change in angulation between pre- and post-treatment condyles should be minimal, unless there is correction in asymmetric patients.

\section{Area Measurements}

Area measurements of the condyle in the axial plane were calculated by making different axial slices in the TMJ module. The condyle in these slices was cut and exported into ImageJ64 to measure the area. This measurement was used as stated by Meng et al. (2008) in comparing TMJ morphology of children and adults: Largest area of the condylar process (LACP) - area of the condyle in its greatest dimension in the axial plane.

This measurement will be compared in pre- and posttreatment images to determine any changes in the area of the left and right condylar processes in these 2 planes. Changes in the condylar head could provide support for TMJ remodeling. 


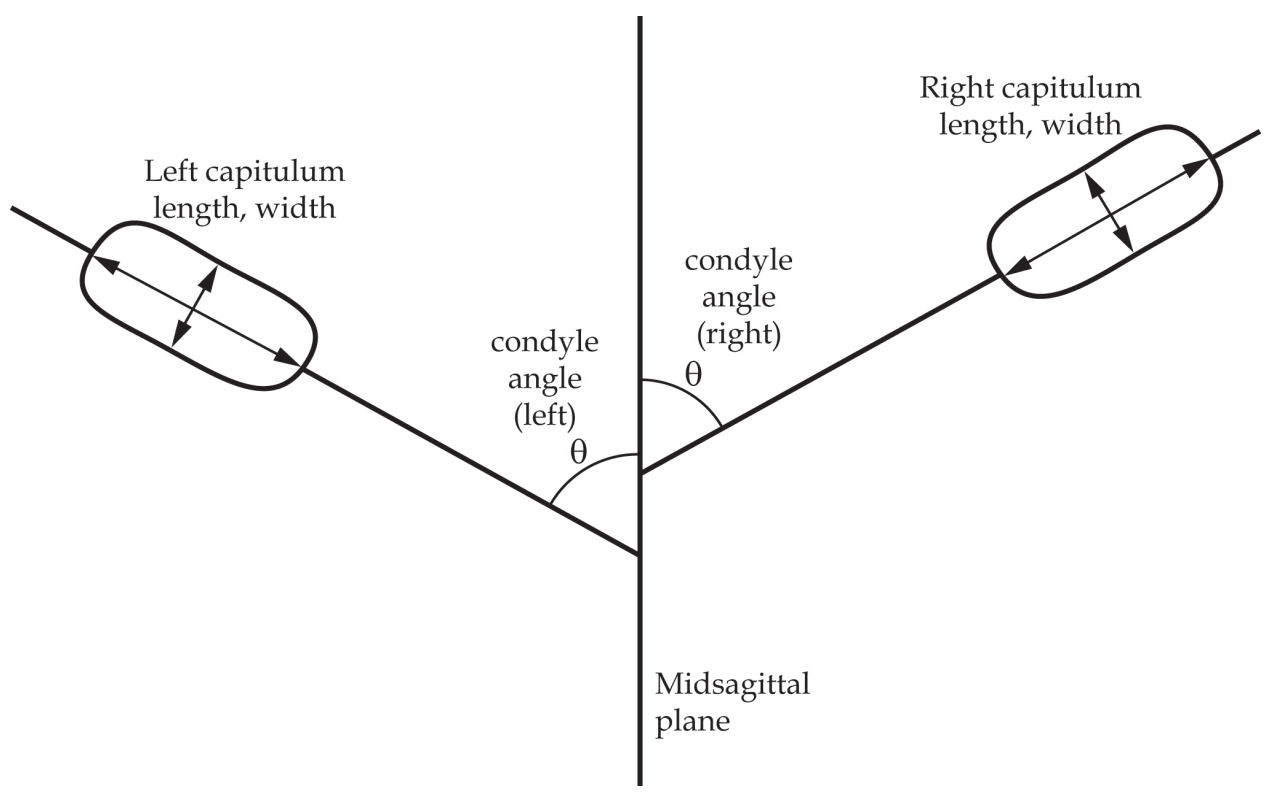

Figure 3-2. Condylar angulation, anteroposterior width of condyles, and mediolateral width of condyles.

\section{Sagittal Dimension}

Sagittal plane reconstruction of the TMJ was done first by referencing the condyles in the axial plane in the TMJ module of Dolphin 11, as stated before in axial reconstruction. The axial image was used to find the axial slice where both the right and left condylar heads are viewed at their greatest area dimension. A reference slice was drawn through the condyle that separated it into equal medial and lateral sections and also accounted for the angle of the condylar head to the midsagittal plane. This slice was processed in both the right and left condyle to produce the proper images for measuring in the "condylar-corrected" sagittal plane for the right and left condyles.

\section{Landmarks}

Most of the landmarks for the sagittal plane were taken from Cho et al. (2009). He and his colleagues used panoramic radiographs to determine condylar differences in temporomandibular osteoarthritic patients and asymptomatic normal patients. These landmarks were used for the corrected sagittal plane measurements.

1. Cd (Condylion): most superior point on condylar head.

2. $\mathrm{Cd}^{\mathrm{O}}$ : most posterior point on condyle on ramus. 
3. $\mathrm{Cd}^{\mathrm{i}}$ : internal point of condyle formed from the perpendicular of the posterior ramus tangent at $\mathrm{Cd}^{\circ}$.

4. $\mathrm{Cd}^{\mathrm{c}}$ : center of condyle between $\mathrm{Co}^{\circ}$ and $\mathrm{Co}^{\mathrm{i}}$.

5. Sigmoid notch (Inc): most inferior aspect of mandible between condyle and coronoid notch.

6. Inc': point describing the level of the sigmoid notch measured perpendicular to the posterior ramus tangent.

The following landmarks were taken from Rodrigues et al. (2009) in evaluating the TMJ in Class II and Class III patients:

1. Articular Eminence (AE): the most inferior point of the articular eminence.

2. External auditory meatus (EAM): circular radiolucency defining the orifice of the external ear.

Ikeda et al. (2009) used the following landmark, superior aspect of glenoid foss (SF), in evaluating ideal condylar position using limited CBCT imaging.

\section{Linear Measurements}

Linear measurements were taken in the sagittal plane using the aforementioned landmarks. The images gathered from the corrected sagittal plane reconstruction were measured in the Dolphin 11 TMJ module with the measurement tool with accurate measurements to $0.1 \mathrm{~mm}$. The following linear measurements used were taken from Rodrigues et al. (2009) (Figure 3-3):

1. Anterior Joint Space (AJS): shortest distance between the most anterior part of condyle and the posterior wall of the articular eminence.

2. Superior Joint Space (SJS): distance from Cd to SF.

3. Posterior Joint Space (PJS): shortest distance between the most posterior part of condyle and the posterior wall of the glenoid fossa.

4. Fossa Width: measured from AE to the most inferior point of the EAM (EAM $\left.{ }^{\mathrm{i}}\right)$.

5. Fossa Depth: measured from SF to the plane formed by the fossa width.

Joint space was measured in pre- and post-treatment images to determine that the condyles were seated properly in the glenoid fossa and that bony apposition of the fossa 


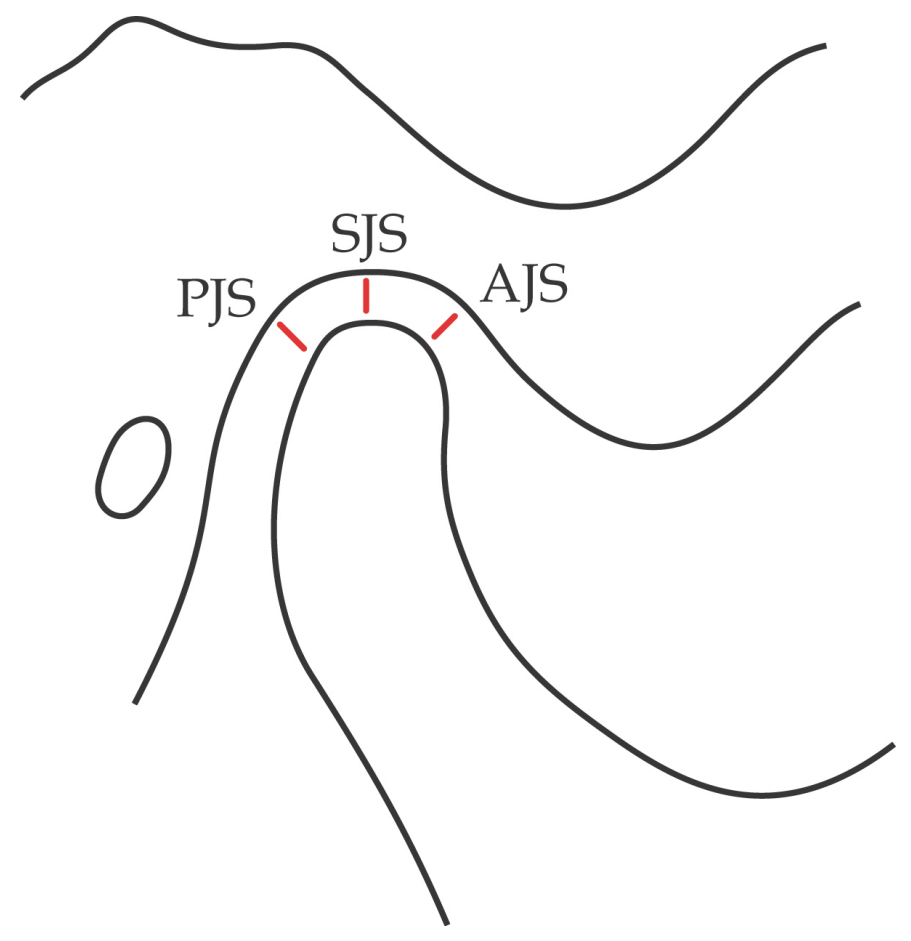

Figure 3-3. Joint spaces between condyle and glenoid fossa. 
has taken place with advancement of the condyle. Fossa width and depth were measured to show any changes of the fossa in relation to the external auditory meatus (Figure 3-4).

The following measurements used were taken from Cho et al. (2009) (Figures 3-5 and 3-6):

1. Condylar Head Width (CHW): measurement from $\mathrm{Cd}^{\circ}$ to $\mathrm{Cd}^{\mathrm{i}}$.

2. Condylar Head Height $(\mathrm{CHH})$ : measurement from $\mathrm{Cd}$ to $\mathrm{Cd}^{\mathrm{c}}$.

3. Condylar Height $(\mathrm{CH})$ : measurement from $\mathrm{Cd}$ to the plane formed from Inc to Inc'.

The following measurements were taken in order to compare the pre- and posttreatment changes seen in the CBCT images taken for each individual: Measurement from $\mathrm{EAM}^{\mathrm{i}}$ to

1. AE.

2. $\mathrm{Cd}^{\mathrm{c}}$.

3. Posterior wall of glenoid fossa measured from PJS.

The external auditory meatus was used as a reference point due to its relatively constant position posterior to the glenoid fossa (Ricketts 1950). These measurements disclosed any advancement seen in the condyles following treatment. If stable advancement of the condyle was seen, there should be a relatively similar increase in all of the linear measurements. Changes seen in condylar position were verified by superimposing pre- and post-treatment images using the EAM to determine the validity of the axially measured condylar advancement.

\section{Angular Measurements}

Angular measurements of the condyles in the sagittal plane were measured by exporting the images into ImageJ64. These angles were used in the condylar-corrected sagittal plane to measure the change of the position of the condylar head and fossa:

1. Articular Tubercle Angle (ATA): angle formed from line connecting the EAM ${ }^{\mathrm{i}}$ to the $\mathrm{AE}$ and the line from the SF to the AE (Figure 3-7).

2. Condylar Head Angle (CHA): angle formed from a line intersecting the condylar process to $\mathrm{Cd}^{\mathrm{c}}$ and the line formed by connecting $\mathrm{Cd}$ to $\mathrm{Cd}^{\mathrm{c}}$.

The ATA dimension disclosed any changes in the posterior slope of the articular eminence (condylar path) due to functional appliance treatment. The CHA indicated 


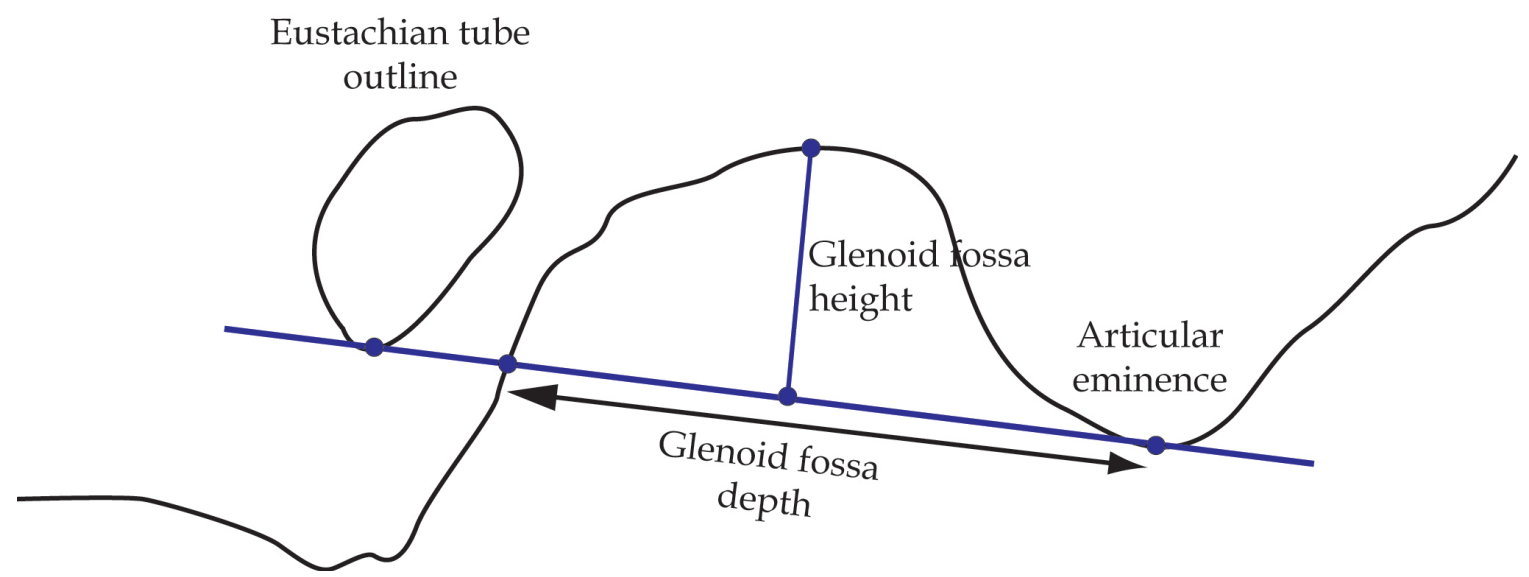

Figure 3-4. Glenoid fossa depth and height.

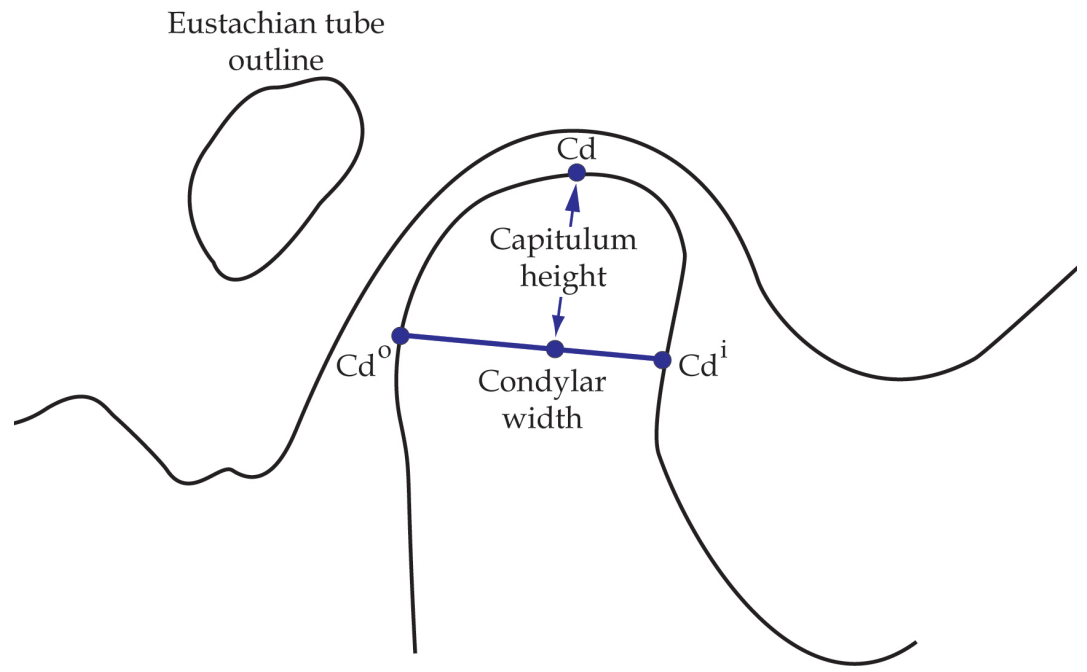

Figure 3-5. Condylar head width and height. 


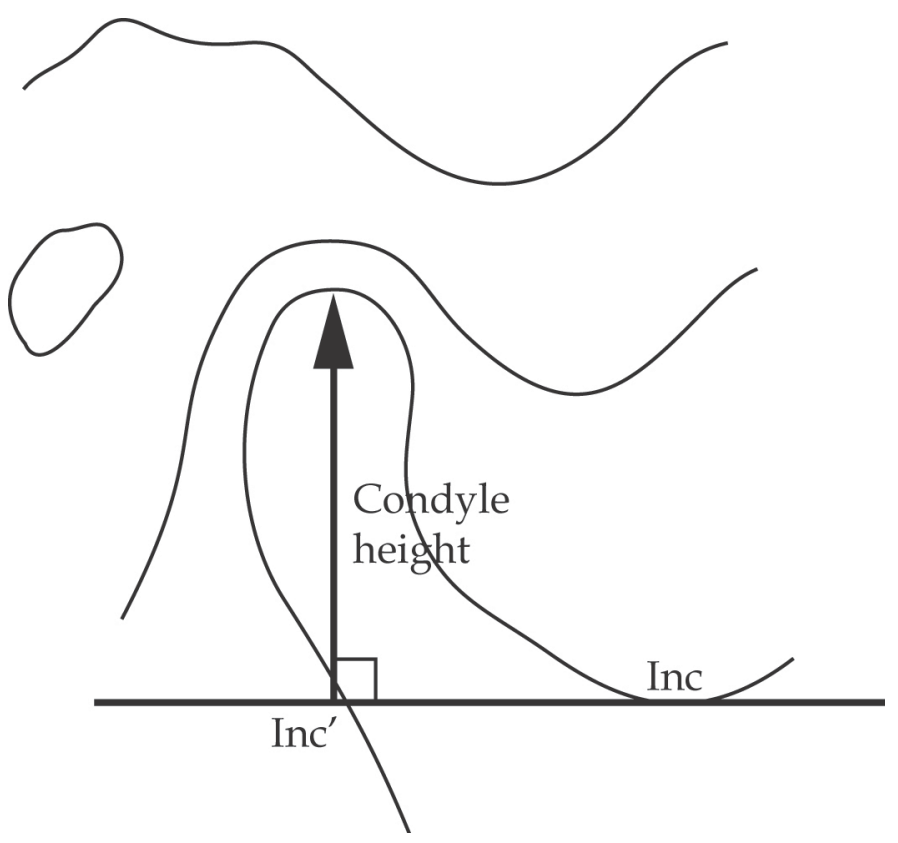

Figure 3-6. Condylar height.

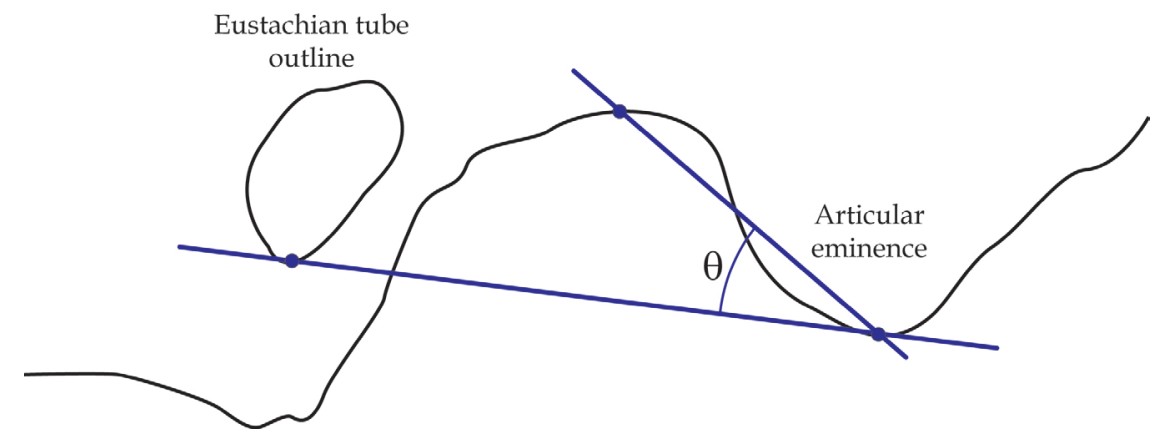

Figure 3-7. Articular tubercle angle (ATA). 
whether a change occurred in the angle of the condylar process due to bony remodeling of the condylar head.

\section{Area Measurements}

Area measurements were constructed in Dolphin 11. Segmentation was used to cut out a specific portion of the CBCT image, and then the cut was exported to ImageJ64 to calculate the area measurements. The following measurements were used to evaluate area measurements in the condylar region (Figure 3-8):

1. Area of condylar process (ACP): measured from the outline of the condyle superior to Inc and Inc'.

2. Area of the Articular Eminence (AAE): measured from the outline of the articular eminence inferior to the SF posteriorly and to the point formed by the plane connecting the SF and most superior point of the EAM $\left(\mathrm{EAM}^{\mathrm{s}}\right)$ on the anterior portion of the articular eminence.

3. Area of the Glenoid Fossa (AGF): measured from the outline of the glenoid fossa superior to the plane formed from the fossa width.

Calculating the areas of these temporomandibular regions gives an understanding of whether TMJ remodeling takes place in functional appliance therapy with the MARA. If stable condylar advancement and remodeling is seen, anticipated in-treatment outcomes should be seen in MARA cases: decreased area of the articular eminence (anterior resorption) and relatively little change in the area of the glenoid fossa. If the AGF has increased, treatment may not be stable due to no posterior bony apposition of
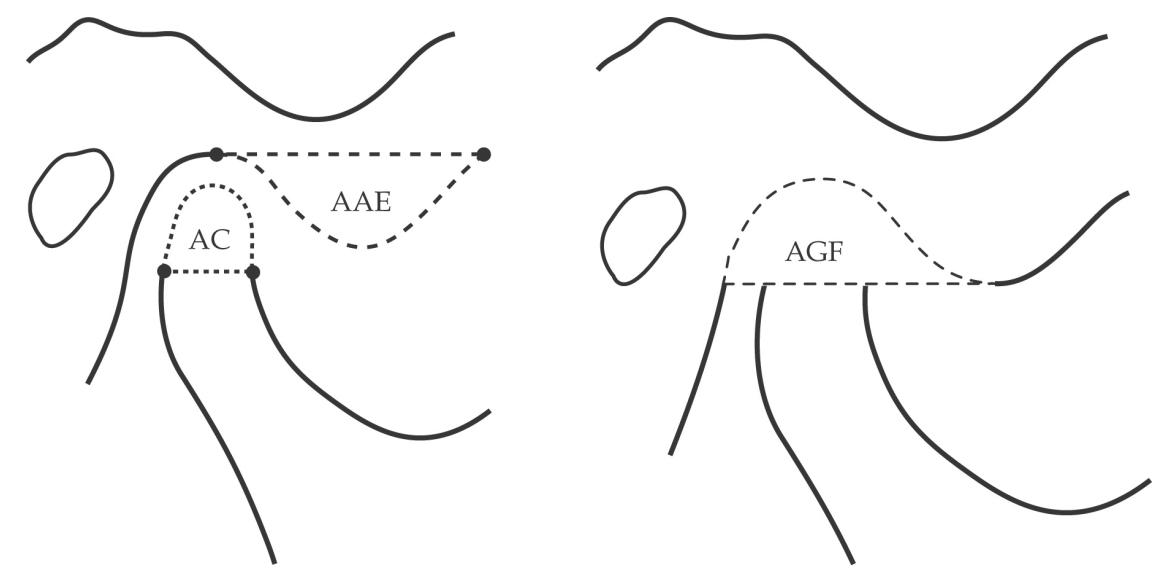

Figure 3-8. Showing the measurements of area of condylar process (AC), area of articular eminence (AAE) and area of glenoid fossa (AGF). 
the glenoid fossa and a "double bite" may be present. The area of the condylar process may or may not change with TMJ remodeling.

\section{Coronal Dimension}

The coronal plane was reconstructed using the above process for determining the sagittal plane. Once the "condylar-corrected" sagittal plane had been constructed in the TMJ module in Dolphin 11, a coronal slice was placed through the widest aspect of each condyle mediolaterally to produce the coronal image.

As previously done in the sagittal plane, measurements were recorded using the measuring tool in the TMJ module of Dolphin 11. These measurements were taken in the coronal plane to determine joint space in the coronal aspect of the TMJ (Figure 3-9):

1. Medial Joint Space (MJS): shortest distance from the most medial aspect of the condylar head to the medial wall of the glenoid fossa.

2. Superior Joint Space (SJS): distance from Cd to SF.

3. Lateral Joint space (LJS): shortest distance from the lateral aspect of the condyle to the lateral wall of the glenoid fossa.

The measurement of these joint spaces has not been previously measured using other conventional forms of radiography. These measurements show any changes to the TMJ in the coronal plane in MARA treatment.

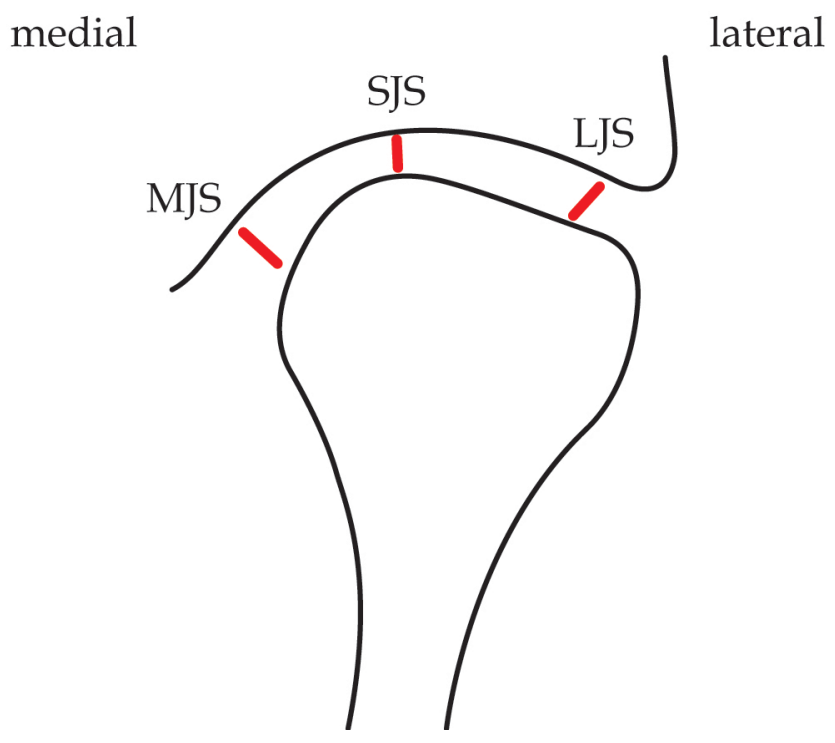

Figure 3-9. Joint space in the coronal plane. 


\section{Lateral Cephalometric Dimension}

A lateral cephalometric image can be constructed in Dolphin 11 from the full CBCT image. First, the image must be oriented to Frankfort Horizontal. The image can then be measured with the Dolphin 11 software.

\section{Landmarks}

The landmarks used on the lateral cephalogram for measurement, as described by Athanasiou (1995), are as follows (Figure 3-10):

1. Nasion $(\mathrm{Na})$ : the most anterior point on the frontonasal suture.

2. Subspinale (A point): the most posterior point on the curvature of the anterior maxilla.

3. Supramentale (B Point): the most posterior point on the curvature of the anterior mandible.

4. Sella (Se): the center of the hypophyseal fossa (sella turcica).

5. Incision superius (Is): the incisal edge of the most anterior maxillary incisor.

6. Incision inferius (Ii): the incisal edge of the most anterior mandibular incisor.

7. Pterygomaxillary Fissure $(\mathrm{Pt})$ : the most superoposterior point of the pterygomaillary fissure.

8. M point: the most posterior point of the mandibular symphysis.

9. U6C: the tip of the mesiobuccal cusp of the maxillary first molar.

10. L6C: the tip of the mesiobuccal cusp of the mandibular first molar.

11. Functional occlusal plane: line representing position and anteroposterior cant of the occlusal plane drawn through the occlusal contacts of the premolars and first molar.

\section{Linear Measurements}

The linear measurements described were measured in Dolphin 11. The following measurements will be taken on pre- and posttreatment images (Figures 3-11 and 3-12): 


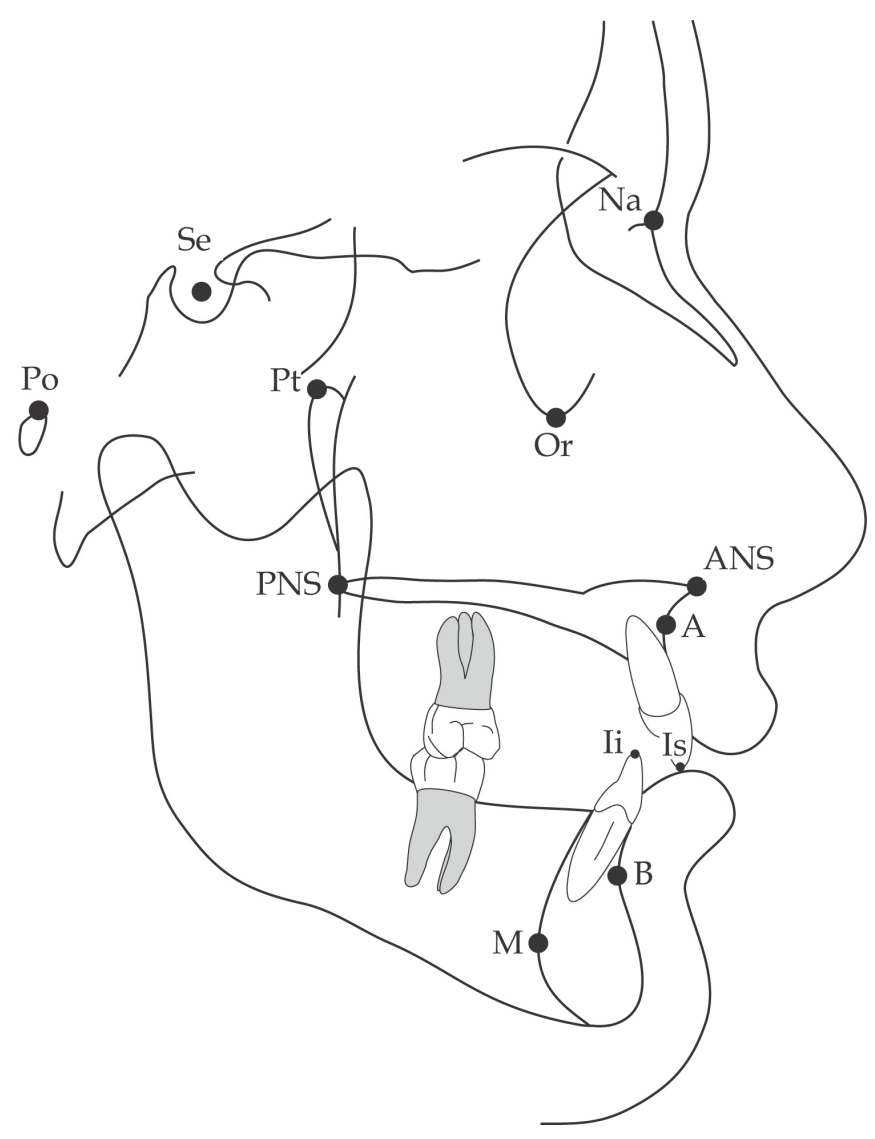

Figure 3-10. Cephalometric landmarks. 

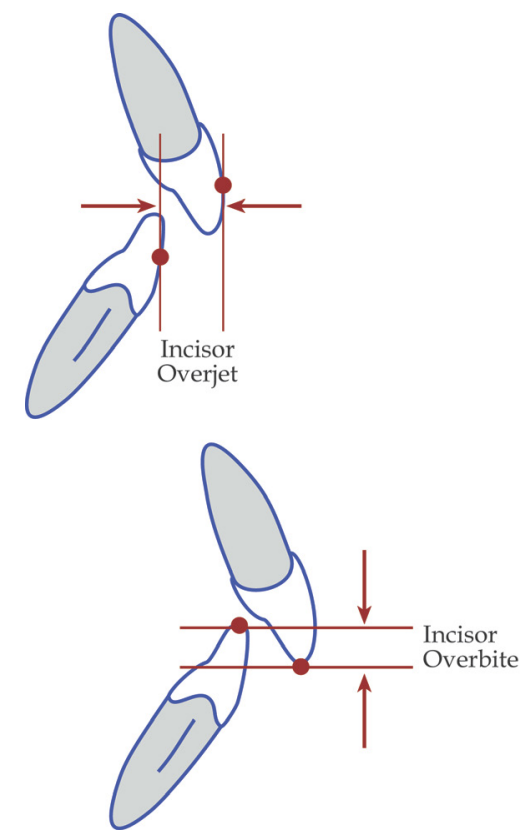

Figure 3-11. Illustration showing incisor overjet and overbite. 


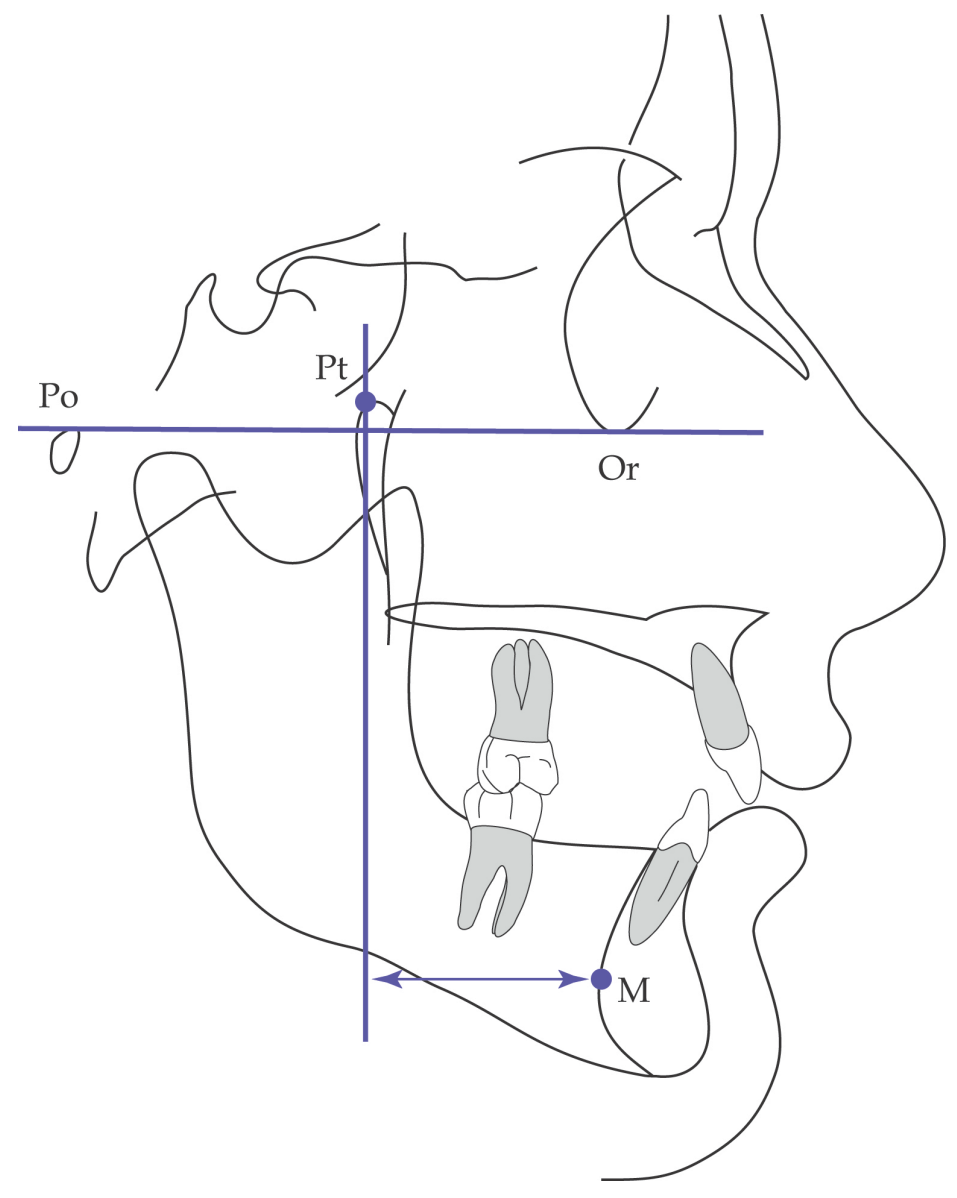

Figure 3-12. Illustration of the cephalometric measurement Pterygoid-Vertical to $M$ point, where $M$ is the distal-most aspect of the mandibular symphysis. 
1. Incisal overjet: the horizontal measurement of the maxillary and mandibular incisal edges as related to the occlusal plane.

2. Incisal overbite: the vertical measurement of the maxillary and mandibular incisors as related to the occlusal plane.

3. Molar relationship: the horizontal measurement of U6 to L6 referenced to the occlusal plane.

4. Total mandibular advancement: linear distance from Sella-V to $m$ point when projected perpendicular to the Frankfort horizontal plane.

Incisal overjet, overbite, and molar relationship were used to evaluate, compare, and equilibrate the two sample groups. Total mandibular advancement was evaluated to show the amount of horizontal advancement of the mandible as related to a stable cranial orthodontic landmark (sella).

\section{Angular Measurements}

Angular measurements were measured in Dolphin 11. These angular measurements were taken from both the pre- and posttreatment examinations:

1. SNA: position of the maxilla to the skull base (Figure 3-13).

2. SNB: position of the mandible to the skull base (Figure 3-14).

3. ANB: relation of the maxilla to the mandible.

These measurements were used to evaluate, equilibrate, and compare the two study groups.

\section{Statistical Design}

In order to remove any bias, subjects' images were categorized, numbered and randomized by an independent evaluator. A separate evaluator recorded all the measurements. Measurements were remeasured on a number of random images (twelve) two months later to calculate intraoperator reliability.

Measurements will be exported into a spreadsheet in Microsoft ${ }^{\circledR}$ Excel 2008 (Microsoft Corporation, Redmond, WA). The spreadsheet was used to combine patient information including demographic information (patient's age, sex, jaw asymmetry, functional or conventional treatment). The measurements were then transferred to the statistical package JMP ${ }^{\circledR} 9.0$ (SAS Institute Inc., Cary, NC). Analysis of covariance 


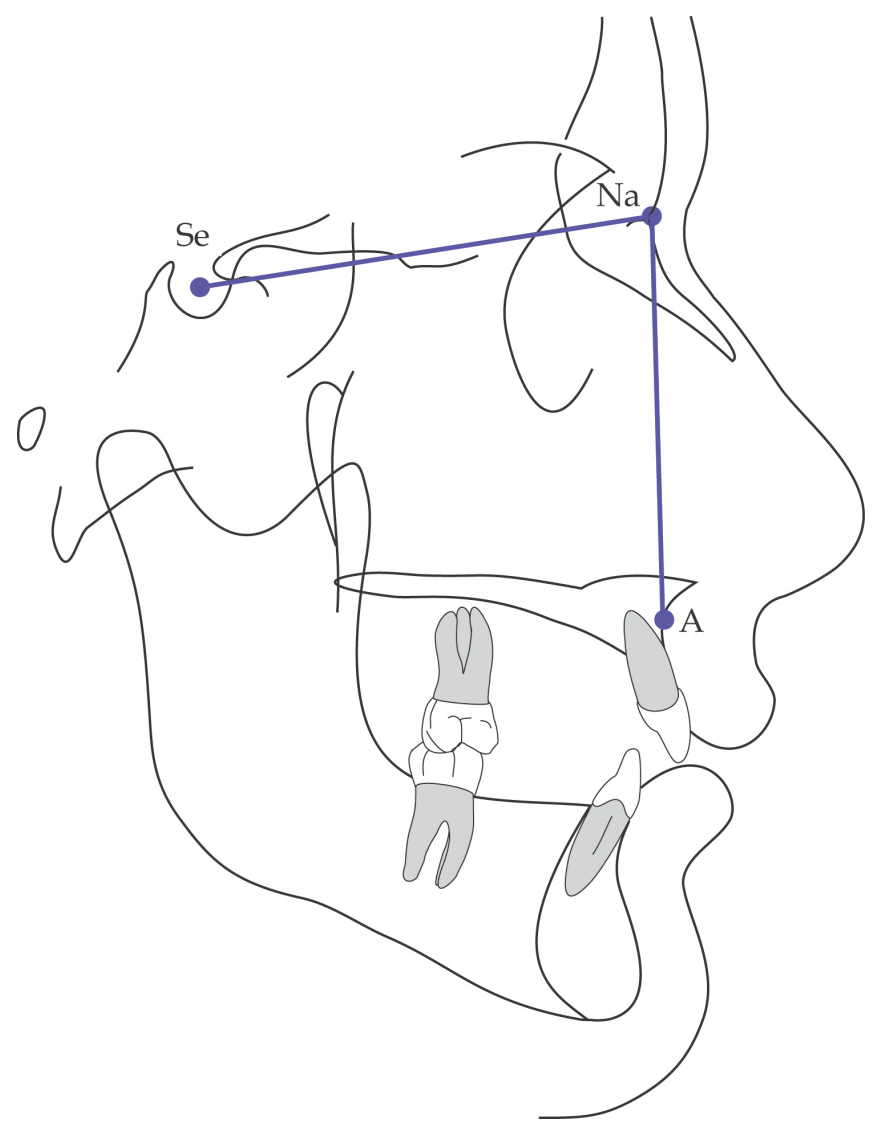

Figure 3-13. Illustration of the cephalometric measurement SNA. 


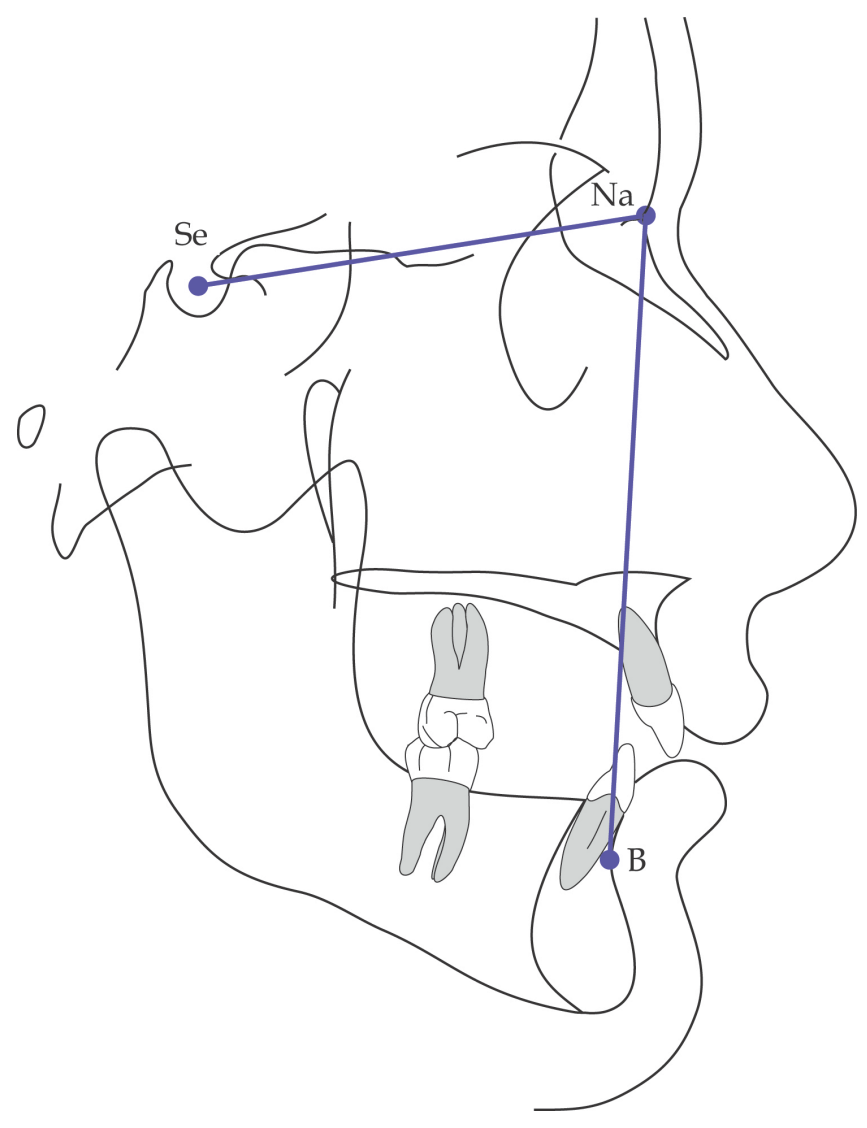

Figure 3-14. Illustration of the cephalometric measurement SNB. 
(ANCOVA) was used to simultaneously test for an age difference (the covariate) while controlling for sexual dimorphism. Appendices A-D show the overall statistical analysis of the study. 


\section{CHAPTER 4. RESULTS}

\section{Initial Group Comparability}

It seemed important early on in the analysis to document the comparability of the malocclusions. That is, at the start of treatment (before any intervention) that the nature of the malocclusions were comparable. To test this, a two-way factorial ANOVA model was used (Table 4-1), where one of the factors was "treatment" (Edgewise mechanics with or without a MARA). The second factor was sex (boy, girl). The full model was calculated, so the interaction effect (treatment-by-sex) was included. There were, then, three $\mathrm{F}$ ratio tests for each of the seven cephalometric variables. It is noteworthy that none of these statistical tests was significant (alpha $=0.05$, two-tail tests). This comprehensive absence of statistical differences argues for the equal conditions of the initial malocclusions between groups.

\section{Intraobserver Repeatability}

It was considered important to quantify the level of intraobserver repeatability (also commonly termed TEM, technical error of measurement) for this study.

The perspective used here is that it is impossible for repeated measurements of the same object to be identical (e.g., Houston 1983; Houston et al. 1986). Differences are due in part to human (operator) differences in landmark selection, personal definitions of the variable - that can change over time - and the level of precision (significant digits) recorded (Houston 1983; Houston et al. 1986). Another component of the repeatability differences is the measuring instrument. All have some fixed level of digits, beyond which they are not reliable, and machines (calipers, computers) do not always measure consistently or equally precisely in all planes of space. Instrument errors actually can be viewed as coming from two sources, random error and systematic error. Random error occurs because our system of measurements is limited to fixed increments. Systematic errors occur because there is a problem with the instrument. As an example, readings from a computer program where the screen setting is distorted or not at the correct screen resolution will produce false values. If calipers have been bent, they will yield systematically larger or smaller values than the correct ones (e.g., Harris and Smith 2009).

It is valuable to quantify the extent of intraobserver reliability (termed Technical Error of Measurement, TEM) because TEM shows the opportunity for imprecision. It is hoped that repeated measurements would be precise and accurate. Precision (repeatability, reproducibility) is a measure of how consistent (close together) measurements are of the same object. Vierira and Corrente (2011, p 488) stated: "By definition, repeatability is the closeness of agreement between successive readings obtained by the same method on the same material and under the same condition (same operator, same apparatus, same setting and same time)." Accuracy is how closely the 
Table 4-1. Two-way factorial ANOVA models testing for comparability of the malocclusions at the initial records examinations.

\begin{tabular}{|c|c|c|c|c|c|c|}
\hline \multirow[b]{2}{*}{ Malocclusion } & \multicolumn{2}{|c|}{ Treatment } & \multicolumn{2}{|c|}{ Patient's Sex } & \multicolumn{2}{|c|}{ Treatment-by-Sex } \\
\hline & F Ratio & P Value & F Ratio & P Value & F Ratio & P Value \\
\hline SNA & 0.00 & 0.9618 & 0.21 & 0.6489 & 0.71 & 0.4028 \\
\hline SNB & 0.01 & 0.9249 & 0.02 & 0.8938 & 0.26 & 0.6093 \\
\hline ANB & 0.10 & 0.7588 & 0.51 & 0.4772 & 0.66 & 0.4191 \\
\hline Overbite & 0.20 & 0.6559 & 0.03 & 0.8572 & 0.03 & 0.8558 \\
\hline Overjet & 0.44 & 0.5114 & 0.00 & 0.9792 & 1.06 & 0.3085 \\
\hline Molar Relation & 0.55 & 0.4619 & 2.92 & 0.0928 & 0.00 & 0.9528 \\
\hline Sella-Vertical-to-M & 0.75 & 0.3900 & 0.21 & 0.6450 & 1.46 & 0.2320 \\
\hline
\end{tabular}


measured values are to the true value. Using the commonly referenced target analogy (Figure 4-1), measurements can be precise but not accurate. Or, they can be accurate but not precise. The goal, of course, is for the measurements to be both precise and accurate, so TEM is small-less than the supposed intergroup differences. This ensures that nonbiological TEM effects do not unduly influence the observed biological differences.

It can be hard to determine a quantity's true value, but the larger the sample size, the closer we can get to the true size.

This section of Results analyzed a set of replicate measurements. From the original set of 64 cases, $12(\sim 20 \%)$ were remeasured several months later while blinded to the subject's original readings. All variables were remeasured on these 12 cases, so there was a sample size of $12 \times 112$ or 1,344 replicated pairs of numbers.

\section{Systematic Error}

One possibility is that the second set of measurements was systematically different from the first, perhaps because the operator's opinion of where a landmark was located had changed during the interim. Matched (paired) t-tests were used to test for this (two-tail tests).

\section{Random Error}

The conventional Dahlberg statistic (Dahlberg 1940) calculated for each variable as:

$$
d=\sqrt{\frac{\sum_{i=1}^{n}\left(X_{1 i}-X_{2 i}\right)^{2}}{2 n}}
$$

where $X_{1 j}$ and $X_{2 j}$ are the two measurements for subject $\mathrm{j}$ and $\mathrm{n}$ is the number of replicated (pairs of) subjects (Dahlberg 1940; Midtgård et al. 1974; Knapp 1992). The difference is squared to make them all positive. This is not the mean difference of the measurement error as sometimes claimed; it is the standard error of the measurement difference (Altman and Bland 1983; Bland and Altman 1996, 1999, 2003).

We put considerable reliance on the Dahlberg statistic (Table 4-2), but we have also been swayed by the arguments of Vierira and Corrente (2011). They point out that the Dahlberg statistic only holds when the readings are (1) independent, (2) identically distributed random variables, and (3) the average of the differences between readings is on average zero.

The first effort here was to evaluate all 112 variables using paired $t$ tests. Paired $t$ tests are more efficient than group comparison $t$ tests when the data-as here-are 

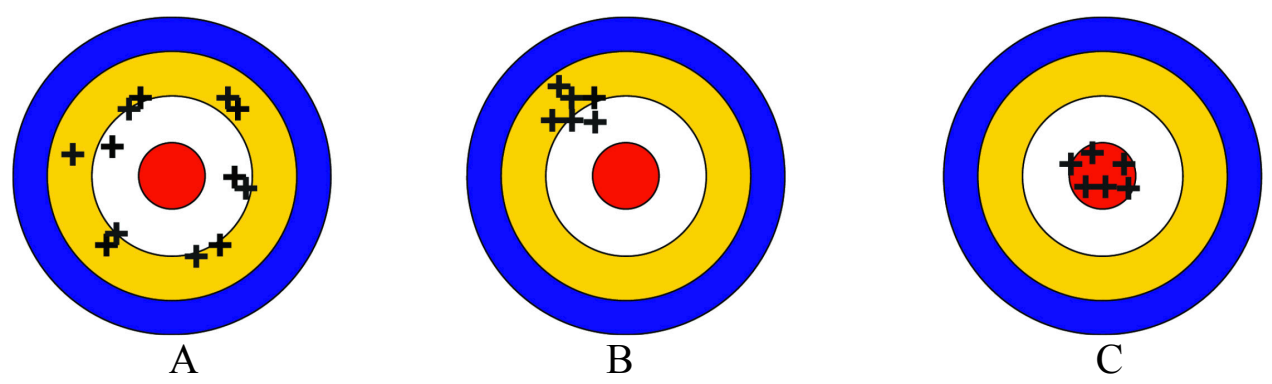

Figure 4-1. A metaphor of a 'bull's eye" characterizes the concepts of precision and accuracy.

(A) The mean of the measurements is close to the center of the bull's eye, which is the true value. These measurements have low repeatability, however, because of their scatter and individual departures from the true value. (B) The measurements are close together (good precision), but all are approximately equally biased from the true value. For example, calipers might be out of kilter, so all measurements are exaggerated by, say, 0.1 $\mathrm{mm}$. (C) Here the measurements are all close to the measurement (high accuracy) and close to one another (high precision). Modified with permission. Harris EF, Smith RN. Accounting for measurement error: A critical but often overlooked process. Arch Oral Biol 2009, 54, Supplement 1:107-17. 
Table 4-2. Listing of the Dahlberg statistic for the 112 variables when remeasured.

\begin{tabular}{|c|c|c|}
\hline Variable & Sample Size & Dahlberg Statistic \\
\hline ANB Final & 12 & 0.1979 \\
\hline ANB Initial & 12 & 0.1791 \\
\hline Axial AP L Final & 12 & 0.1756 \\
\hline Axial AP L Initial & 12 & 0.3007 \\
\hline Axial AP R Final & 12 & 0.3240 \\
\hline Axial AP R Initial & 12 & 0.3096 \\
\hline Axial APCD Final & 12 & 0.4168 \\
\hline Axial APCD Initial & 12 & 0.4865 \\
\hline Axial CA L Final & 12 & 2.0164 \\
\hline Axial CA L Initial & 12 & 1.7548 \\
\hline Axial CA R Final & 12 & 1.2450 \\
\hline Axial CA R Initial & 12 & 1.3563 \\
\hline Axial LACP L Final & 12 & 5.5403 \\
\hline Axial LACP L Initial & 12 & 3.2763 \\
\hline Axial LACP R Final & 12 & 5.6657 \\
\hline Axial LACP R Initial & 12 & 3.9814 \\
\hline Axial ML L Final & 12 & 0.3149 \\
\hline Axial ML L Initial & 12 & 0.3464 \\
\hline Axial ML R Final & 12 & 0.4052 \\
\hline Axial ML R Initial & 12 & 0.4098 \\
\hline Axial MLCP L Final & 12 & 0.5730 \\
\hline Axial MLCP L Initial & 12 & 0.4486 \\
\hline Axial MLCP R Final & 12 & 0.7613 \\
\hline Axial MLCP R Initial & 12 & 0.4349 \\
\hline Condylar LJS L Final & 12 & 0.1190 \\
\hline Condylar LJS L Initial & 12 & 0.1137 \\
\hline Condylar LJS R Final & 12 & 0.1291 \\
\hline Condylar LJS R Initial & 12 & 0.0707 \\
\hline Condylar MJS L Final & 12 & 0.1671 \\
\hline Condylar MJS L Initial & 12 & 0.1173 \\
\hline Condylar MJS R Final & 12 & 0.1208 \\
\hline Condylar MJS R Initial & 12 & 0.0913 \\
\hline Condylar SJS L Final & 12 & 0.1429 \\
\hline Condylar SJS L Initial & 12 & 0.1339 \\
\hline Condylar SJS R Final & 12 & 0.1486 \\
\hline Condylar SJS R Initial & 12 & 0.1099 \\
\hline Mesial Molar Relation Final & 12 & 0.3878 \\
\hline Mesial Molar Relation Initial & 12 & 0.3851 \\
\hline
\end{tabular}

Continued 
Table 4-2. Continued.

\begin{tabular}{|c|c|c|}
\hline Variable & Sample Size & Dahlberg Statistic \\
\hline Overbite Final & 12 & 0.3060 \\
\hline Overbite Initial & 12 & 0.3298 \\
\hline Overjet Final & 12 & 0.2598 \\
\hline Overjet Initial & 12 & 0.2380 \\
\hline Sagittal AAE L Final & 12 & 0.2380 \\
\hline Sagittal AAE L Initial & 12 & 9.7407 \\
\hline Sagittal AAE R Final & 12 & 7.8852 \\
\hline Sagittal AAE R Initial & 12 & 6.1272 \\
\hline Sagittal ACP L Final & 12 & 3.9894 \\
\hline Sagittal ACP L Initial & 12 & 3.5918 \\
\hline Sagittal ACP R Final & 12 & 3.6309 \\
\hline Sagittal ACP R Initial & 12 & 2.9266 \\
\hline Sagittal AGF L Final & 12 & 5.8100 \\
\hline Sagittal AGF L Initial & 12 & 3.7269 \\
\hline Sagittal AGF R Final & 12 & 7.8119 \\
\hline Sagittal AGF R Initial & 12 & 6.0979 \\
\hline Sagittal AJS L Final & 12 & 0.0842 \\
\hline Sagittal AJS L Initial & 12 & 0.1000 \\
\hline Sagittal AJS R Final & 12 & 0.0979 \\
\hline Sagittal AJS R Initial & 12 & 0.1137 \\
\hline Sagittal ATA L Final & 12 & 4.7394 \\
\hline Sagittal ATA L Initial & 12 & 1.6549 \\
\hline Sagittal ATA R Final & 12 & 0.6889 \\
\hline Sagittal ATA R Initial & 12 & 0.9813 \\
\hline Sagittal CH L Final & 12 & 0.1720 \\
\hline Sagittal CH L Initial & 12 & 0.1732 \\
\hline Sagittal CH R Final & 12 & 0.2951 \\
\hline Sagittal CH R Initial & 12 & 0.2318 \\
\hline Sagittal CHA L Final & 12 & 1.1915 \\
\hline Sagittal CHA L Initial & 12 & 2.3665 \\
\hline Sagittal CHA R Final & 12 & 1.4627 \\
\hline Sagittal CHA R Initial & 12 & 1.4663 \\
\hline Sagittal CHH L Final & 12 & 0.1225 \\
\hline Sagittal CHH L. Initial & 12 & 0.1541 \\
\hline Sagittal CHH R Final & 12 & 0.1658 \\
\hline Sagittal CHH R Initial & 12 & 0.1720 \\
\hline
\end{tabular}

Continued 
Table 4-2. Continued.

\begin{tabular}{|c|c|c|}
\hline Variable & Sample Size & Dahlberg Statistic \\
\hline Sagittal CHW L Final & 12 & 0.1443 \\
\hline Sagittal CHW L Initial & 12 & 0.1683 \\
\hline Sagittal CHW R Final & 12 & 0.1323 \\
\hline Sagittal CHW R Initial & 12 & 0.1528 \\
\hline Sagittal EAM-AE L Final & 12 & 0.3007 \\
\hline Sagittal EAM-AE L Initial & 12 & 0.3142 \\
\hline Sagittal EAM-AE R Final & 12 & 0.2598 \\
\hline Sagittal EAM-AE R Initial & 12 & 0.2806 \\
\hline Sagittal EAM-C L Final & 12 & 0.1594 \\
\hline Sagittal EAM-C L Initial & 12 & 0.1936 \\
\hline Sagittal EAM-C R Final & 12 & 0.1990 \\
\hline Sagittal EAM-C R Initial & 12 & 0.2776 \\
\hline Sagittal EAM-P L Final & 12 & 0.1190 \\
\hline Sagittal EAM-P L Initial & 12 & 0.1354 \\
\hline Sagittal EAM-P R Final & 12 & 0.1339 \\
\hline Sagittal EAM-P R Initial & 12 & 0.1458 \\
\hline Sagittal FD L Final & 12 & 0.1173 \\
\hline Sagittal FD L Initial & 12 & 0.1080 \\
\hline Sagittal FD R Final & 12 & 0.1620 \\
\hline Sagittal FD R Initial & 12 & 0.1683 \\
\hline Sagittal FW L Final & 12 & 3.1643 \\
\hline Sagittal FW L Initial & 12 & 0.3937 \\
\hline Sagittal FW R Final & 12 & 0.2951 \\
\hline Sagittal FW R Initial & 12 & 0.2723 \\
\hline Sagittal MJS L Final & 12 & 0.1307 \\
\hline Sagittal MJS L Initial & 12 & 0.1099 \\
\hline Sagittal MJS R Final & 12 & 0.1080 \\
\hline Sagittal MJS R Initial & 12 & 0.1000 \\
\hline Sagittal PJS L Final & 12 & 0.0890 \\
\hline Sagittal PJS L Initial & 12 & 0.1061 \\
\hline Sagittal PJS R Final & 12 & 0.1190 \\
\hline Sagittal PJS R Initial & 12 & 0.1696 \\
\hline Sella-Vertical-M Final & 12 & 0.7228 \\
\hline Sella-Vertical-M Initial & 12 & 1.1583 \\
\hline SNA Final & 12 & 0.5319 \\
\hline SNA Initial & 12 & 0.3629 \\
\hline SNB Final & 12 & 0.4619 \\
\hline SNB Initial & 12 & 0.2739 \\
\hline
\end{tabular}


related. Efficiency is defined as the likelihood of finding a statistically significant difference when one exists. Appendix $\mathbf{C}$ lists the relevant descriptive statistics and the paired $t$ tests for the double determinations.

We created Bland-Altman plots for the statistically significant variables. Of the 112 variables, $8(8 / 112=7.1 \%)$ were statistically significant at an unadjusted alpha level of 0.05 (Table 4-3). This level is too liberal because of the multiple comparison problem. The common over-correction of Bonferroni level of significance would be $0.05 / 112$ or 0.000446 , and none of the differences is significant at this level. Indeed, by paired t-test (two-tail) the difference with the smallest probability (0.0022) was Sagittal EAM-P R at the initial examination. Importantly, even this "large" difference only differed between measurement sessions by an average of $0.16 \mathrm{~mm}$, which we consider safely unimportant clinically. These significant differences were assessed from paired $t$ tests. In a complementary but different manner, Figures 4-2 through 4-9 are provided. These Bland-Altman plots tested for a significant dependency between size of the difference between paired measurements ( $\mathrm{X}$ axis) and the mean size of the variable ( $\mathrm{Y}$ axis). None of these plots was significant statistically.

\section{Least Squares Means}

You probably have heard the term "least squares means." Least squares means (LS Means) are actually a sort of statistical jargon. Least square means is actually referred to as marginal means (or sometimes EMM, estimated marginal means). In an analysis of covariance model, they are the group means after having controlled for a covariate (i.e., holding it constant at some typical value of the covariate, such as its mean value).

For example, consider some of the CBCT variables discussed here, like condyle size. This size (linear or area) can be used as a covariate because the condyle grows

Table 4-3. Statistics of the eight statistically significant (alpha $=0.05)$ variables when testing for systematic differences between intraobserver repeatability sessions.

\begin{tabular}{lccc}
\hline \multicolumn{1}{c}{ Variable } & Mean Difference & Paired t Test & P Value \\
\hline ANB Initial & & & \\
Axial MLCP R Final & -0.1750 & -3.17 & 0.0089 \\
Condylar LJS L Final & -0.6750 & 2.67 & 0.0218 \\
Mesial Molar Relation Initial & 0.1167 & 3.19 & 0.0086 \\
Overbite Final & 0.3500 & 2.78 & 0.0179 \\
Sa AJS L Final & 0.2833 & 2.25 & 0.0460 \\
Sagittal EAM-P R Initial & 0.0750 & 2.69 & 0.0210 \\
Sagittal FW L Initial & 0.1583 & 3.98 & 0.0022 \\
\hline
\end{tabular}




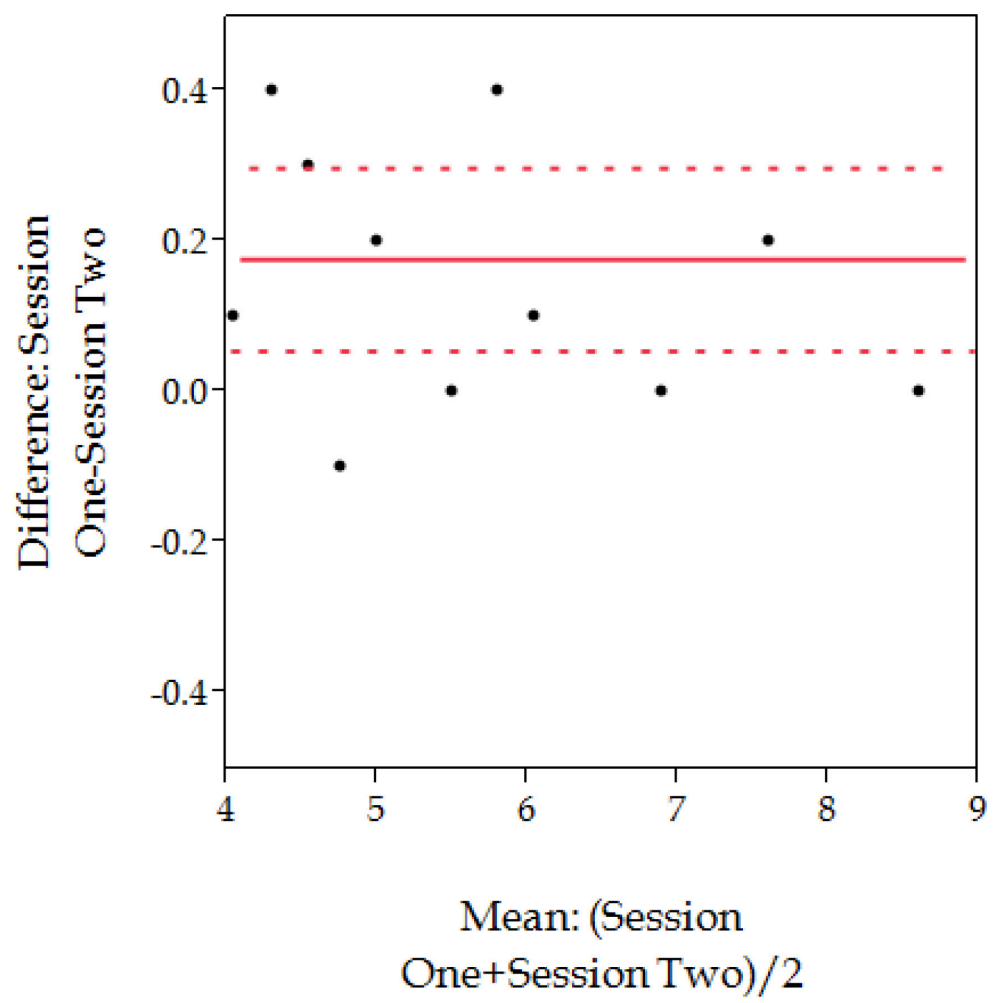

Figure 4-2. Bland-Altman plot for the angle ANB at the initial examination.

Dotted lines are the $95 \%$ confidence limits of the least-squares regression line, which did not differ significantly from zero. 


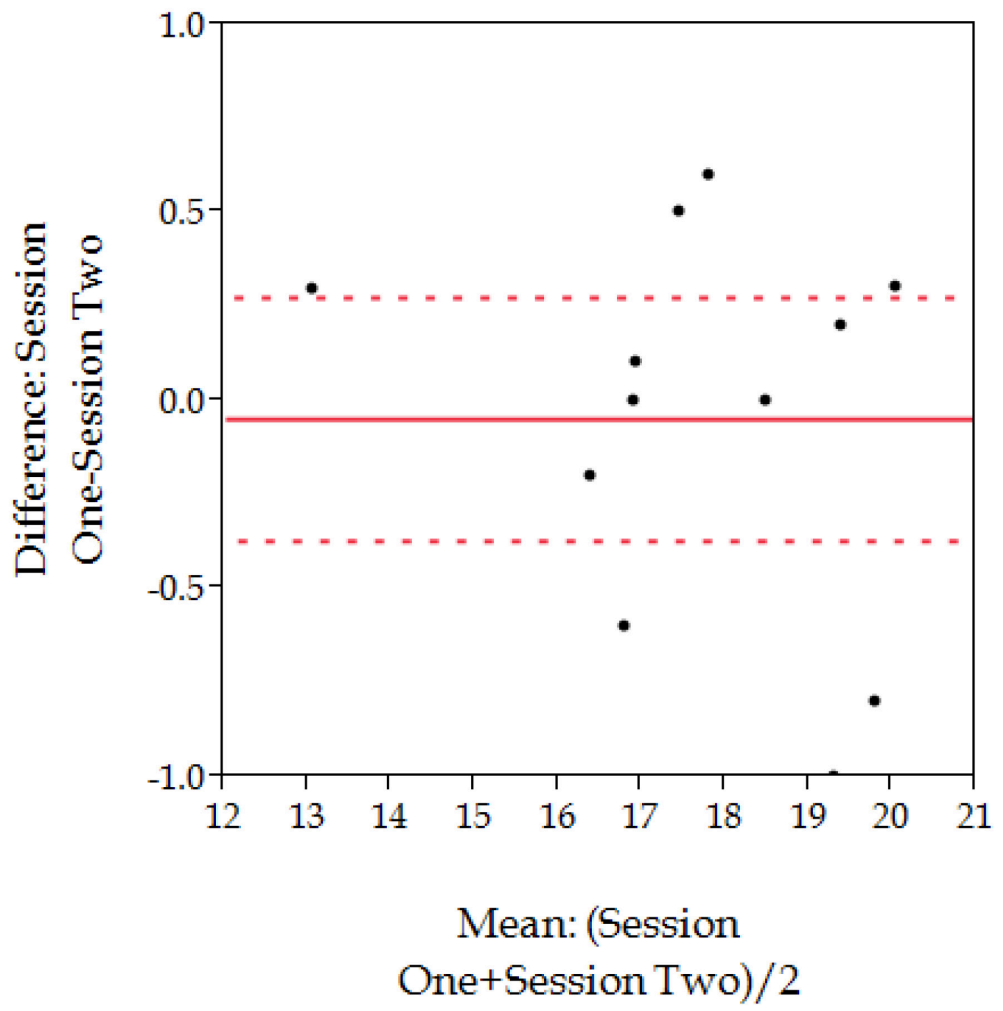

Figure 4-3. Bland-Altman plot for MLCP Right at the final examination.

Dotted lines are the $95 \%$ confidence limits of the least-squares regression line, which did not differ significantly from zero. 


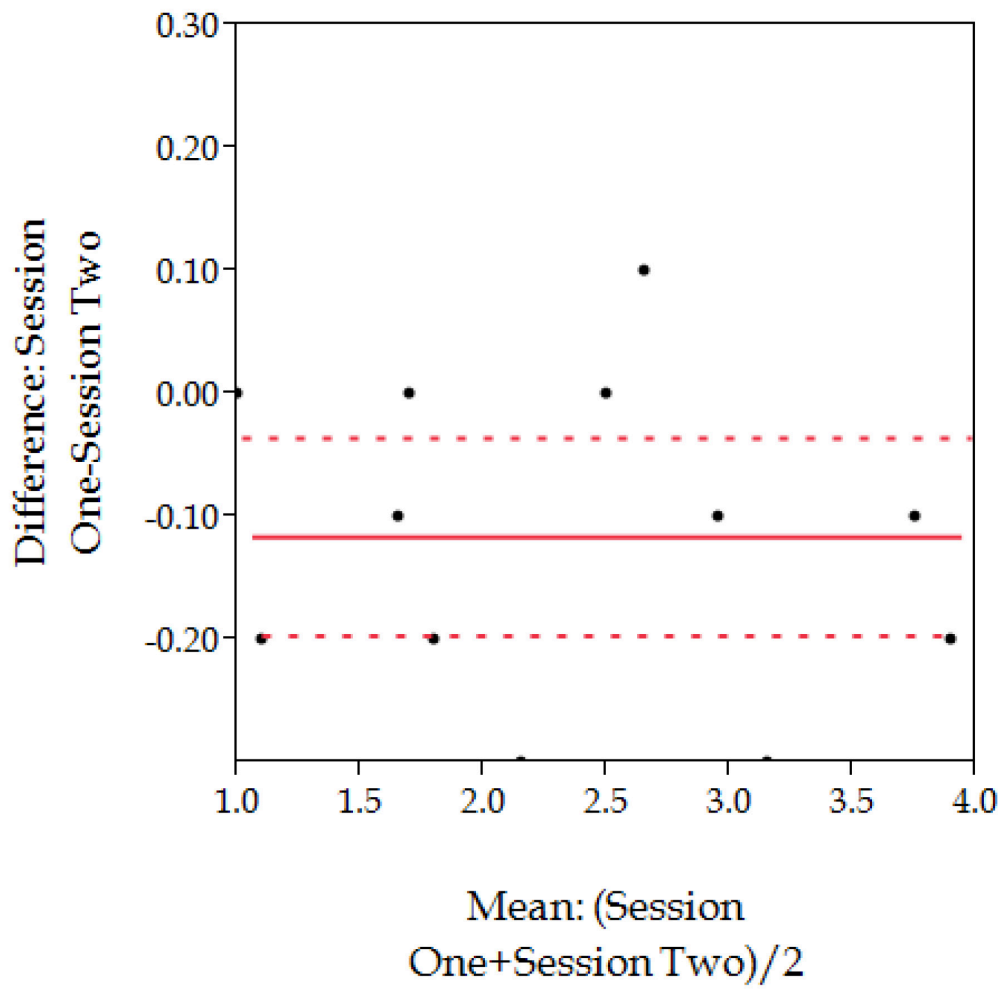

Figure 4-4. Bland-Altman plot for Condyle LJS Left at the final examination.

Dotted lines are the $95 \%$ confidence limits of the least-squares regression line, which did not differ significantly from zero. 


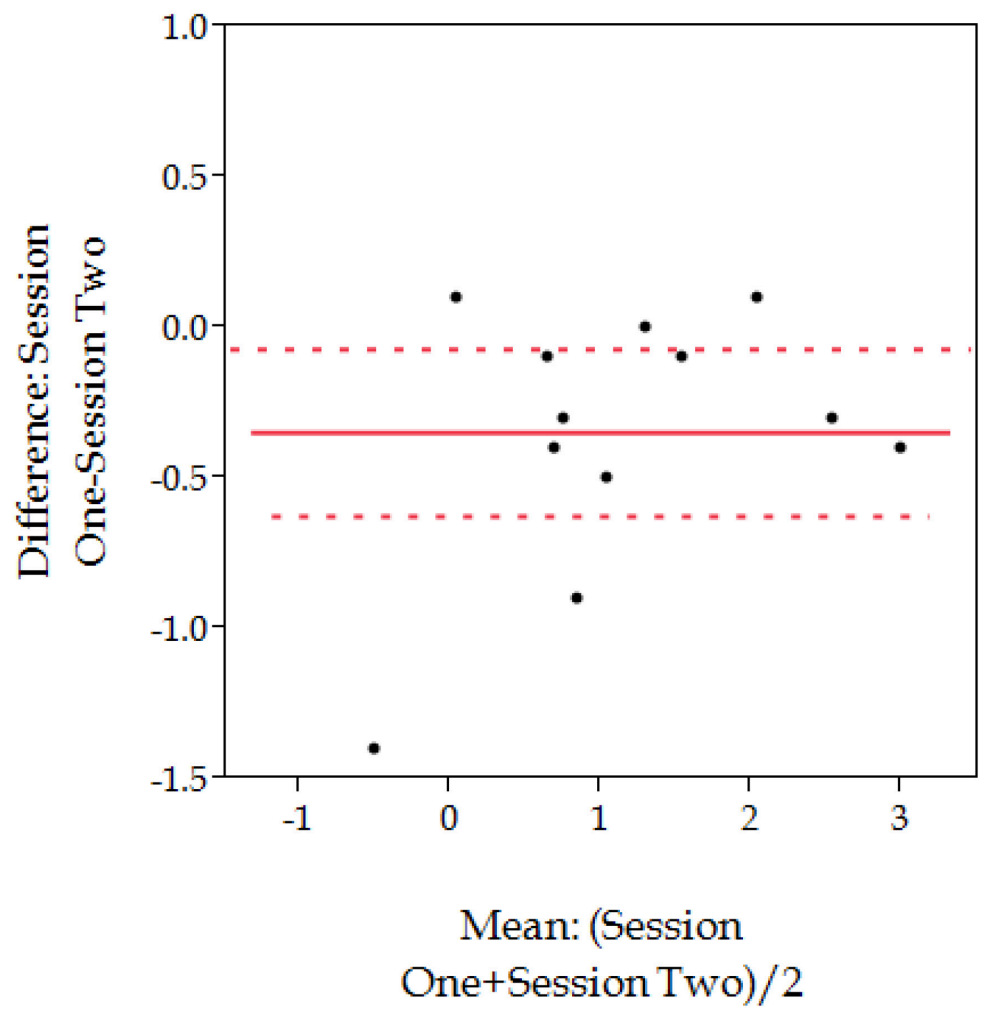

Figure 4-5. Bland-Altman plot for Mesial Molar Relationship at the initial examination.

Dotted lines are the $95 \%$ confidence limits of the least-squares regression line, which did not differ significantly from zero. 


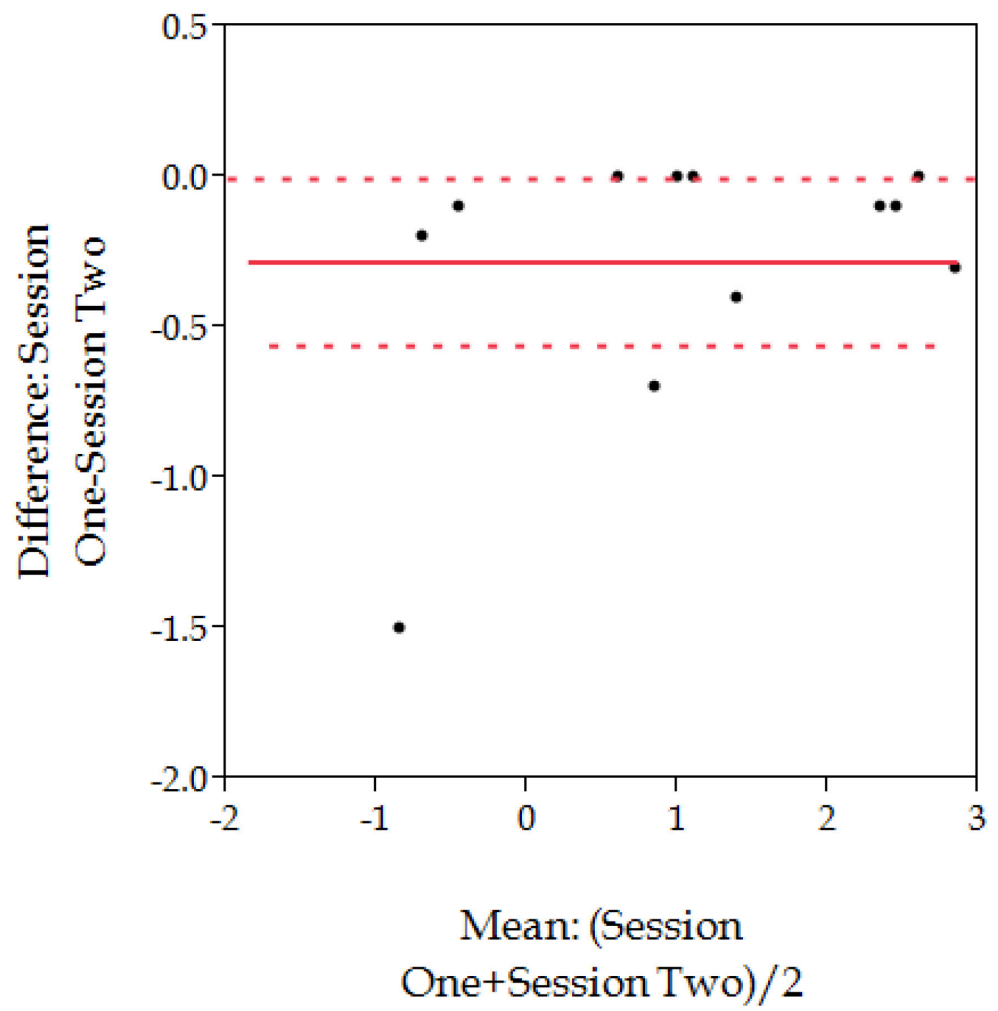

Figure 4-6. Bland-Altman plot for Overbite at the final examination.

Dotted lines are the $95 \%$ confidence limits of the least-squares regression line, which did not differ significantly from zero. 


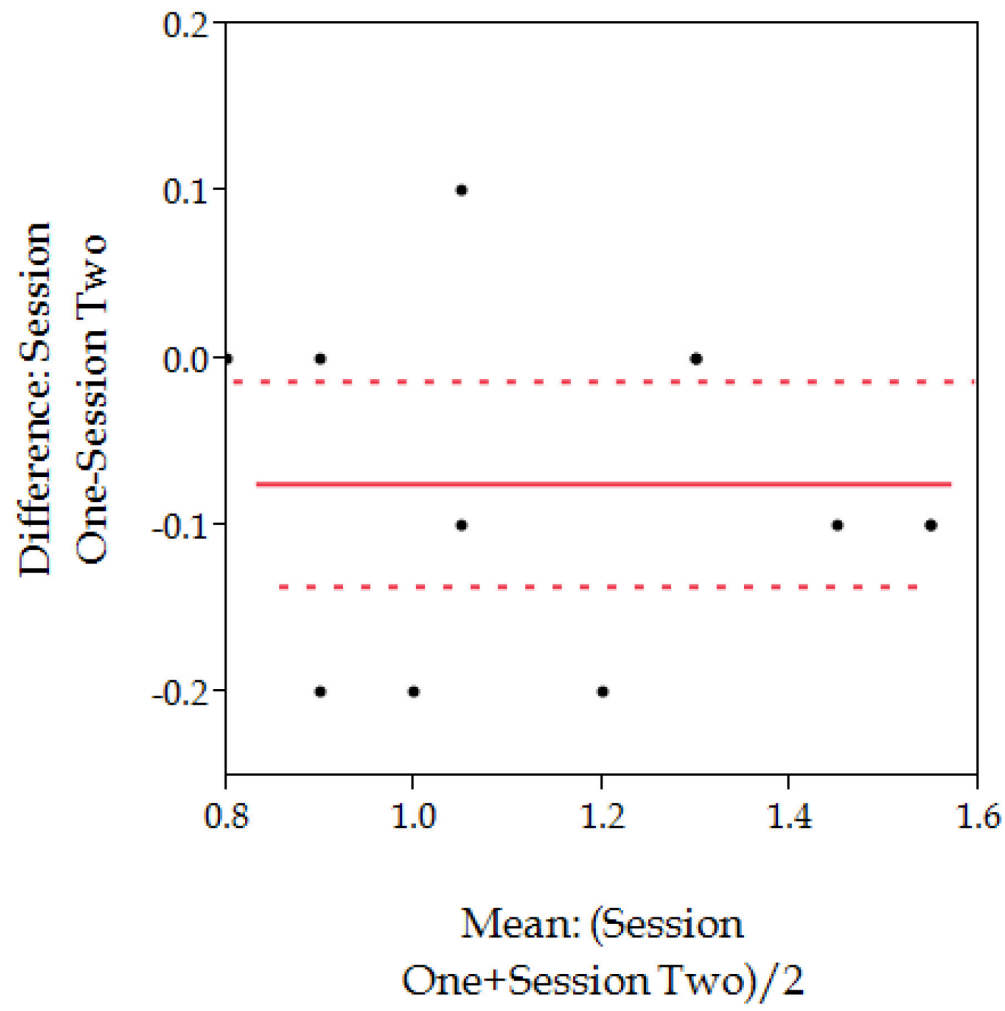

Figure 4-7. Bland-AItman plot for AJS Left at the final examination.

Dotted lines are the $95 \%$ confidence limits of the least-squares regression line, which did not differ significantly from zero. 


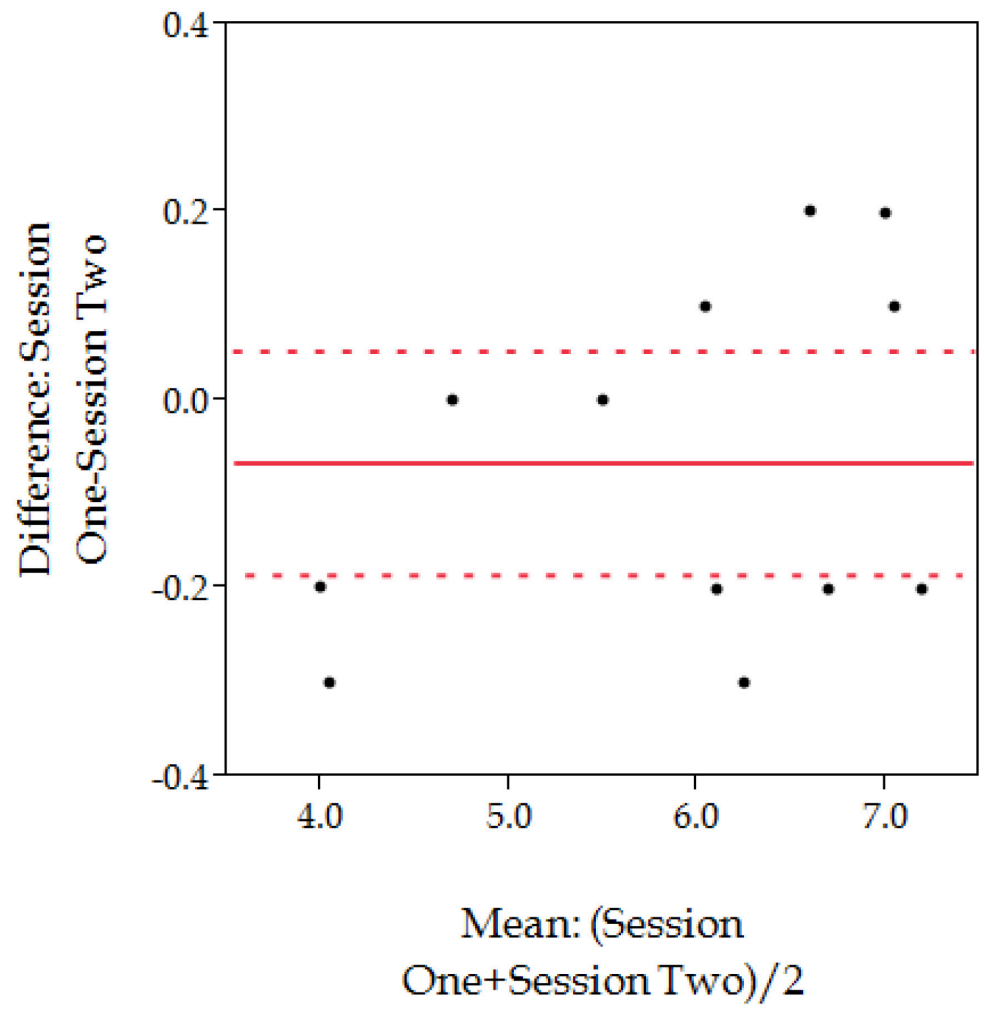

Figure 4-8. Bland-Altman plot for Sagittal EAM-P Right at the initial examination.

Dotted lines are the $95 \%$ confidence limits of the least-squares regression line, which did not differ significantly from zero. 


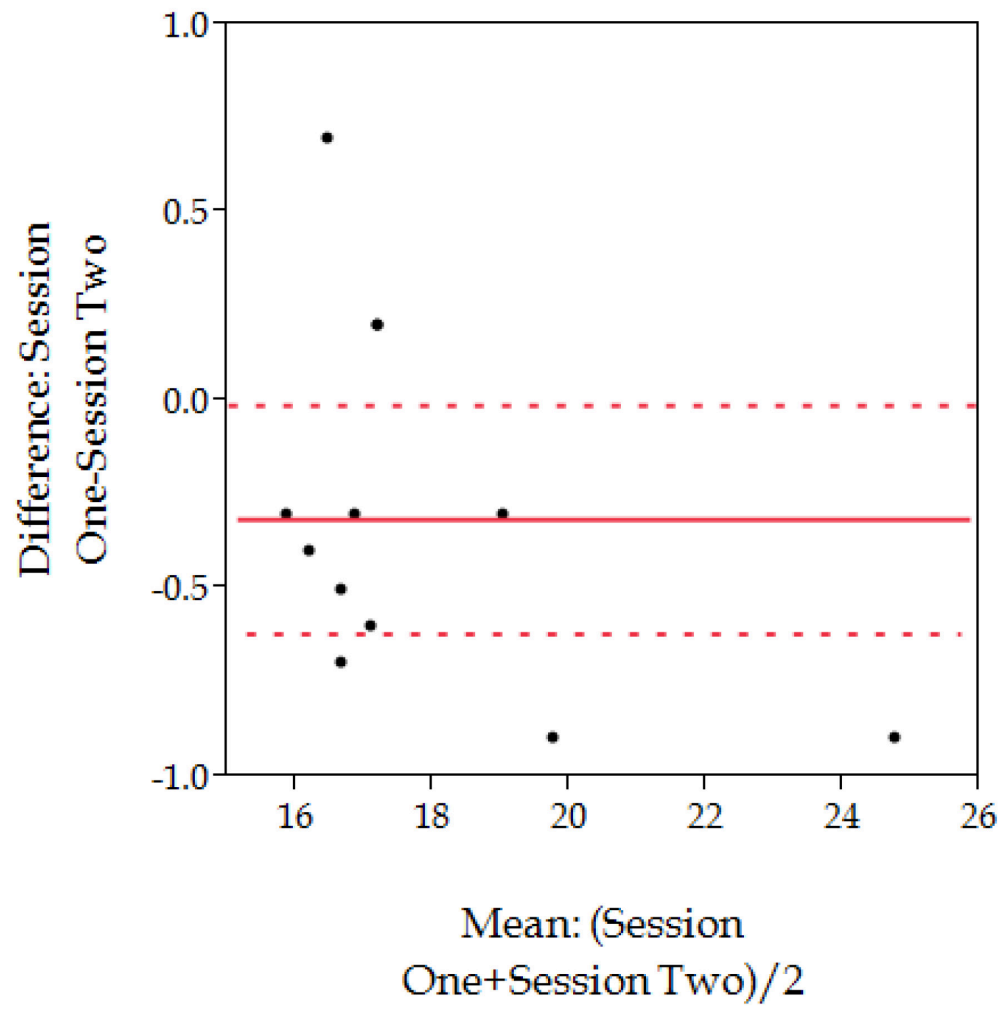

Figure 4-9. Bland-Altman plot for Sagittal FW Left at the initial examination.

Dotted lines are the $95 \%$ confidence limits of the least-squares regression line, which did not differ significantly from zero. 
(enlarges) with the age of the patient. The same thing occurs with using patient age since children and adolescents typically enlarge with age (e.g., stature and body weight). The shortcoming of using ordinary means, then, is that there is the presumption that everyone in the samples is the same age (and the same size). Eliminating inter-individual variation plus changes that occur as a function of age is difficult. Suppose, in theory, that condylar size doubles from 5 to 15 years of age. Then, if the ages at treatment were different between samples, the investigator would be hard pressed to distinguish between pharyngeal sizes due to age compared to differences due to different malocclusions.

This problem can be solved statistically by using least squares means in place of the ordinary means. LS means picks a convenient age (commonly the sample mean) and transforms all of the cases to that same age (or whatever the covariate is in the model). Thus, if skull size or condylar dimensions change with the age of the patient, LS means corrects for this, expressing the variables as if the covariate were constant and the same between samples. This permits direct (unbiased) comparisons of the sizes of the dependent variable across the samples.

From the ANCOVA model used here (Appendix B), the LS means have been listed for all of the variables. The LS means are reported separately for the two sexes (boys, girls) and between the four treatment-by-sex combinations (Appendix C). In unbalanced designs with more than one effect, the arithmetic mean for a group may not accurately reflect the "typical" response for that group, since it does not take other effects into account.

Means and LS means will be the same if the design is balanced (equal number of cases per sample) and there are no missing data. In the present study there are no missing data, but the sample sizes are unequal (boys versus girls and the unequal sample sizes of the two treatments). LS means from different ANOVA models will be different because they have been adjusted for different effects.

\section{Treatment-Clinic Differences}

The research design used here selected the cases treated with Edgewise-Alone mechanics from one clinic (in Tennessee) and cases treated with Edgewise mechanics in combination with a MARA from another clinic (in Kansas). There was no overlap of orthodontists between clinics. As such, treatment mechanics and orthodontic office ("clinic") were confounded. It was not possible to attribute differences solely to mechanics or clinic. One exception was the significant "treatment" differences found at the initial examination. Since no treatment had been delivered, initial differences were due to geographical and/or sampling differences from the two patient populations (TN versus $\mathrm{KS}$ ).

A brute-force method of detecting statistically significant differences in the samples was used. Each variable was tested - at the start of treatment, at the end of treatment, and the in-treatment difference (calculated as the starting condition minus the 
final condition, so a positive change signifies an increase during treatment). The variables ( $\mathrm{k}=112$ dimensions) assessed in this study (see Methods) were a combination of midline and paired dimensions. That is, some conventional midline cephalometric landmarks were used (Sella, A Point, B Point) where there was no pairing. For the dimensions that depended on these midline landmarks, each $\mathrm{CBCT}$ variable was subjected to a two-way factorial analysis of variance (ANOVA), where one factor was method of treatment (Edgewise mechanics with or without MARA) and the second factor was sex of the patient (boy, girl). Both factors are fixed effects, and the full ANOVA model involved three simultaneous F-ratios, namely (1) treatment, (2) sex, and (3) the treatment-by-sex interaction effect.

The other dimensions were paired in that both the left and right temporomandibular joints in each subject were measured. Analysis of these paired (left, right) dimensions called for a more complex, mixed ANOVA model. There was the repeated measure of "side" in addition to treatment and sex tests. There were also between-subjects and within-subject factors that made this a mixed ANOVA model with separate error terms. The between-subject factors were (1) treatment (same as clinic), (2) sex of the patient, and (3) the treatment-by-sex interaction effect. The four within-subject factors were (1) side (left and right temporomandibular joints), (2) side-by-treatment interaction (3) side-by-sex interaction, and (4) the second-order interaction effect, sideby-treatment-by-sex. In all, then, there were 7 simultaneous F-ratios tested for each paired dimension (Appendix B).

The conventional alpha (0.05) was used throughout to gauge statistical significance. This raises the problem of multiple comparisons - elevated chance of finding a significant difference, but that seemed preferable to missing noteworthy differences. The first step in interpreting this plethora of statistical results was to scan the 112 ANOVA models and collect the significant treatment effects $(\mathrm{alpha}=0.05)$. The resulting 25 significant F-ratios are listed in Table 4-4. It seemed safe to assume that any "Treatment" differences that occurred when the initial examinations were performed were more correctly interpreted as geographical (TN versus KS) differences in these samples of the populations. Among the 25 significant tests, there were 6 such instances. These are Sagittal MJS Initial, Sagittal FD Initial, Sagittal CH Initial, Sagittal ATA Initial, Sagittal ACP Initial, and Sagittal AGF Initial. It is interesting that several of these dimensions involve sagittal measurements, but we are unsure at this juncture what this signifies, if anything. The "most significant" of the 25 variables are graphed and described here (those with probability values less than 0.0001 ). The treatment change in the angle SNA are graphed in Figure 4-10.

These Class II cases had Class II division 1 malocclusions, so the typical reduction seen in SNA was anticipated. Of interest significantly greater reduction was achieved in the Edgewise-Alone series. This may be a problem of having to treat the wrong jaw, however. Class II division 1 malocclusions most commonly present with mandibular retrognathia, which optimally would be treated by enhancing mandibular growth (if that were an option), not restricting maxillary growth. 
Table 4-4. List of the statistically significant treatment differences abstracted from the Appendix of ANOVA results (alpha = 0.05).

\begin{tabular}{|c|c|c|}
\hline Variable & Significant Factor & P Value \\
\hline 1. Change in SNA & Treatment & $<0.0001$ \\
\hline 2. Change in SNB & Treatment & 0.0031 \\
\hline 3. Mesial Molar Relation Final & Treatment & 0.0009 \\
\hline 4. Sella-Vertical-to-M Final & Treatment & 0.0008 \\
\hline 5. Change in Sella-Vertical-to-M & Treatment & $<0.0001$ \\
\hline 6. Change in Axial AP & Treatment & 0.0437 \\
\hline 7. Axial LACP Final & Treatment & 0.0437 \\
\hline 8. Change in Axial LACP & Treatment & 0.0437 \\
\hline 9. Sagittal MJS Initial & Treatment & 0.0001 \\
\hline 10. Change in Sagittal MJS & Treatment & 0.0137 \\
\hline 11. Sagittal FW Initial & Side-by-Treatment & 0.0288 \\
\hline 12. Sagittal FW Final & Side-by-Treatment & 0.0006 \\
\hline 13. Sagittal FD Initial & Treatment & 0.0126 \\
\hline 14. Change in Sagittal CHW & Treatment-by-Sex & 0.0009 \\
\hline 15. Sagittal CH Initial & Treatment & 0.0018 \\
\hline 16. Change in Sagittal CH & Treatment & 0.0020 \\
\hline 17. Sagittal EAM-AE Final & Side-by-Treatment & $<0.0001$ \\
\hline 18. Sagittal ATA Initial & Treatment & $<0.0001$ \\
\hline 19. Sagittal ATA Final & Treatment & 0.0056 \\
\hline 20. Change in Sagittal ATA & Treatment & 0.0005 \\
\hline 21. Sagittal CHA Final & Treatment-by-Sex & 0.0382 \\
\hline 22. Sagittal ACP Initial & Treatment & 0.0042 \\
\hline 23. Sagittal ACP Final & Treatment & 0.0004 \\
\hline 24. Sagittal AGF Initial & Side-by-Treatment & 0.0071 \\
\hline 25. Change in Sagittal AGF & Treatment & 0.0035 \\
\hline
\end{tabular}




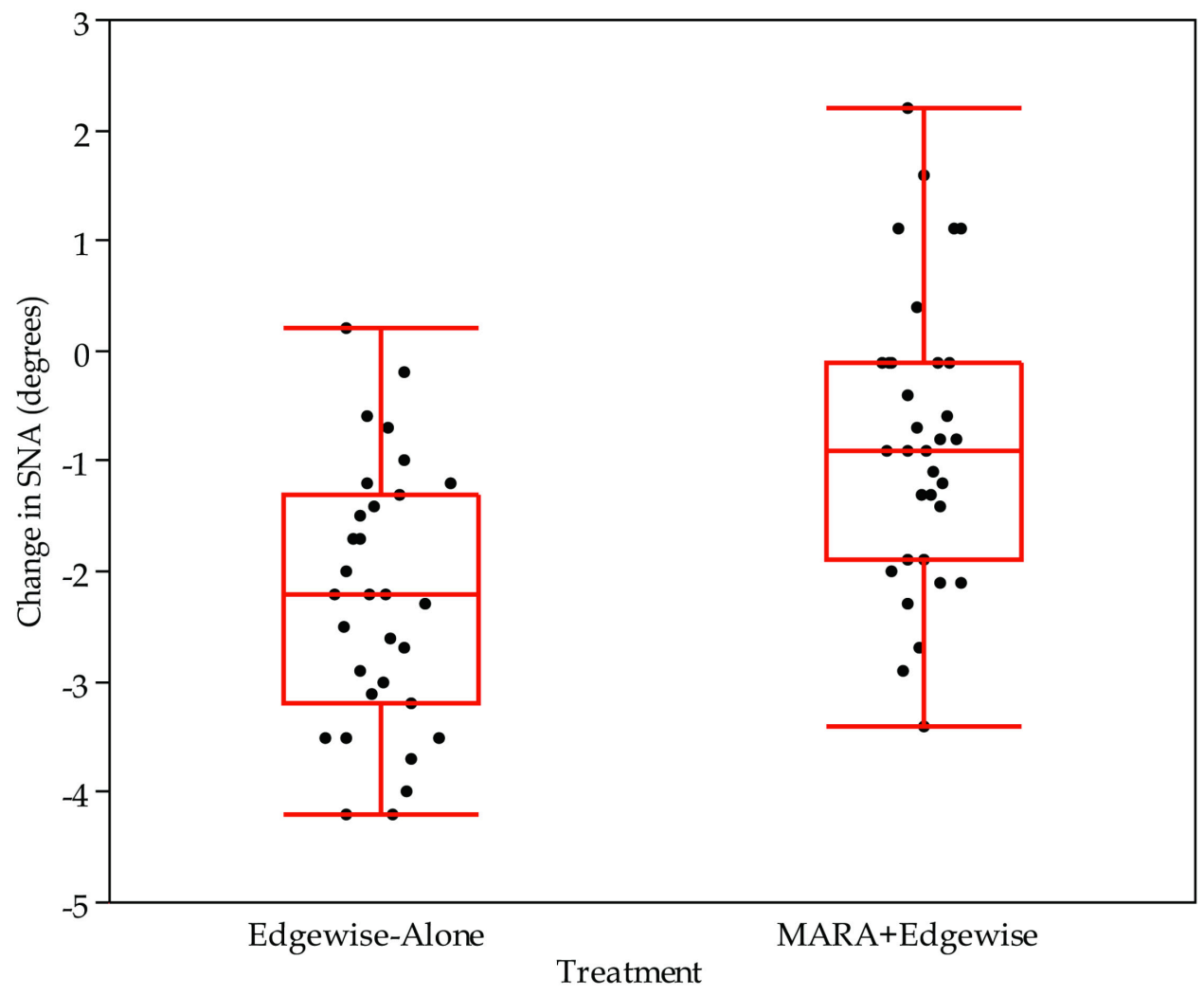

Figure 4-10. Box plots of the in-treatment changes in the angle SNA. 
Examining the interesting changes in the angle SNB (Figure 4-11), there is a significant difference by treatment. In the Edgewise-Alone sample, the angle got smaller, though the median was less than a degree. In the MARA+Edgewise group, the median came forward a bit (roughly $1 / 2 \mathrm{~mm}$ in the graph). Statistically, the difference between treatments achieved significance $(\mathrm{P}=0.0031)$. Inspection of just this dimension suggests that the MARA does aide in repositioning the mandible forward.

In looking at the differences in the axial anteroposterior measurement of the condyle (Figure 4-12), there is a significant difference by treatment. In both the Edgewise-Alone and MARA+Edgewise group, the linear measurement decreased, but the difference between treatments achieved significance $(\mathrm{P}=0.0437)$. This shows more decreasing change of the condylar head in the MARA group versus the Edgewise-Alone group.

The next significant dimension, Axial LACP, is interesting in that this dimension was significant between treatments both at final examinations and the in-treatment changes while being similar at the initiation of treatment. These results are graphed in Figures 4-13 and 4-14. With the AP dimension decreasing more in the MARA+Edgewise group, the assumption that the Edgewise alone sample would have a greater increase was validated with these data.

The Sagittal MJS dimension was significantly larger in the MARA+Edgewise (Kansas) sample at the start of treatment (Figure 4-15). Since no orthodontic work had been done at these initial records, this site difference would seem to be attributable solely to geographical (population) differences of the patient pools and/or to case selection.

In addition to the start of treatment, Sagittal MJS also differed significantly in the amounts of in-treatment change (Figure 4-16). There was little change in this dimension in the MARA+Edgewise (Kansas) sample, but the mode increased significantly in the Edgewise-Alone (Tennessee) sample. The graph also shows that a few (four) samples in the Edgewise-Alone group changed a lot (about $2 \mathrm{~mm}$ ).

The next pair of significant treatment differences was Sagittal FD Initial and Sagittal CH Initial. Since both dimensions differed at the start of treatment, they are dealt with together. Figure 4-17 lists the results, by treatment, for Sagittal FD Initial and Figure 4-18 is for Sagittal CH Initial.

The Sagittal $\mathrm{CH}$ dimension was larger in the MARA+Edgewise group at the start of treatment (Figure 4-18) and it also increased significantly more during treatment, also in the MARA+Edgewise group (Figure 4-19). It cannot be determined whether the greater change was due to treatment instead of a population (Kansas versus Tennessee) difference in intrinsic growth patterns.

At both the start and end of treatment the dimension EAM-AE was larger in the Edgewise-Alone (Tennessee) sample. This is graphed for the start of treatment in Figure 4-20, and at the end of treatment in Figure 4-21. The amounts of in-treatment were 


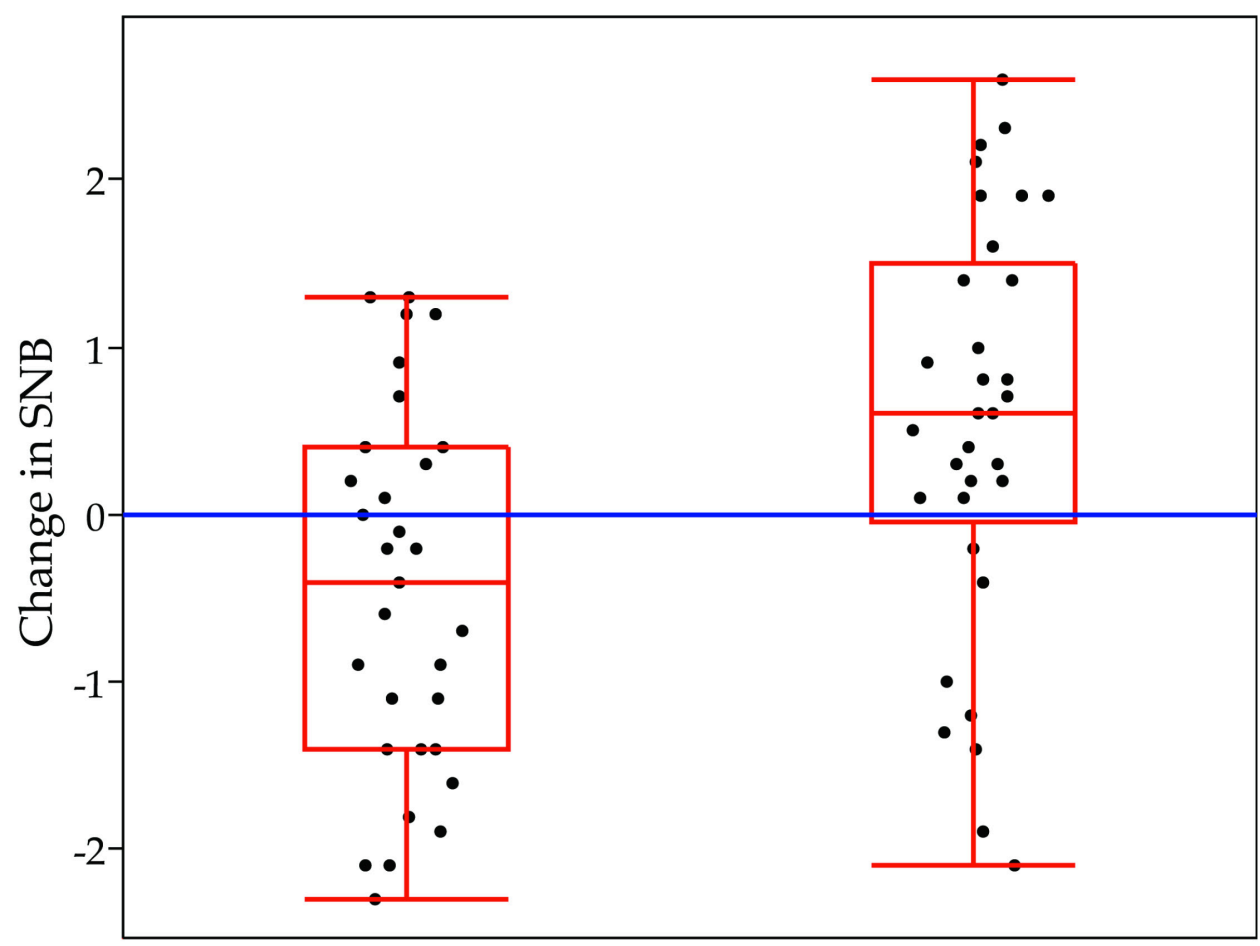

Edgewise-Alone MARA+Edgewise

Treatment

Figure 4-11. Box plots of the changes in SNB, by treatment.

The horizontal blue line is positioned at zero. 


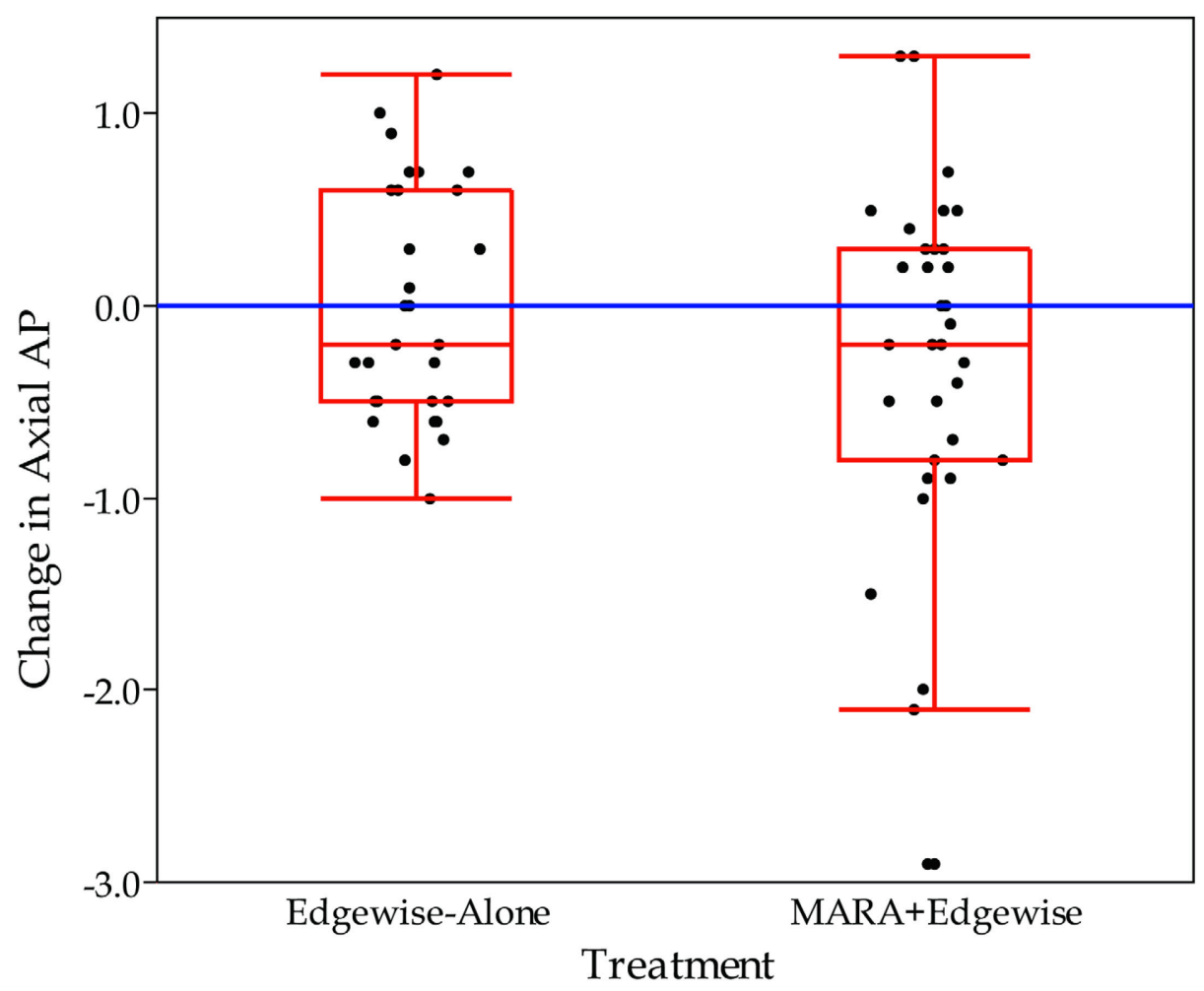

Figure 4-12. Box plots of the in-treatment changes in Axial AP, by treatment.

The blue horizontal line was placed at zero. 


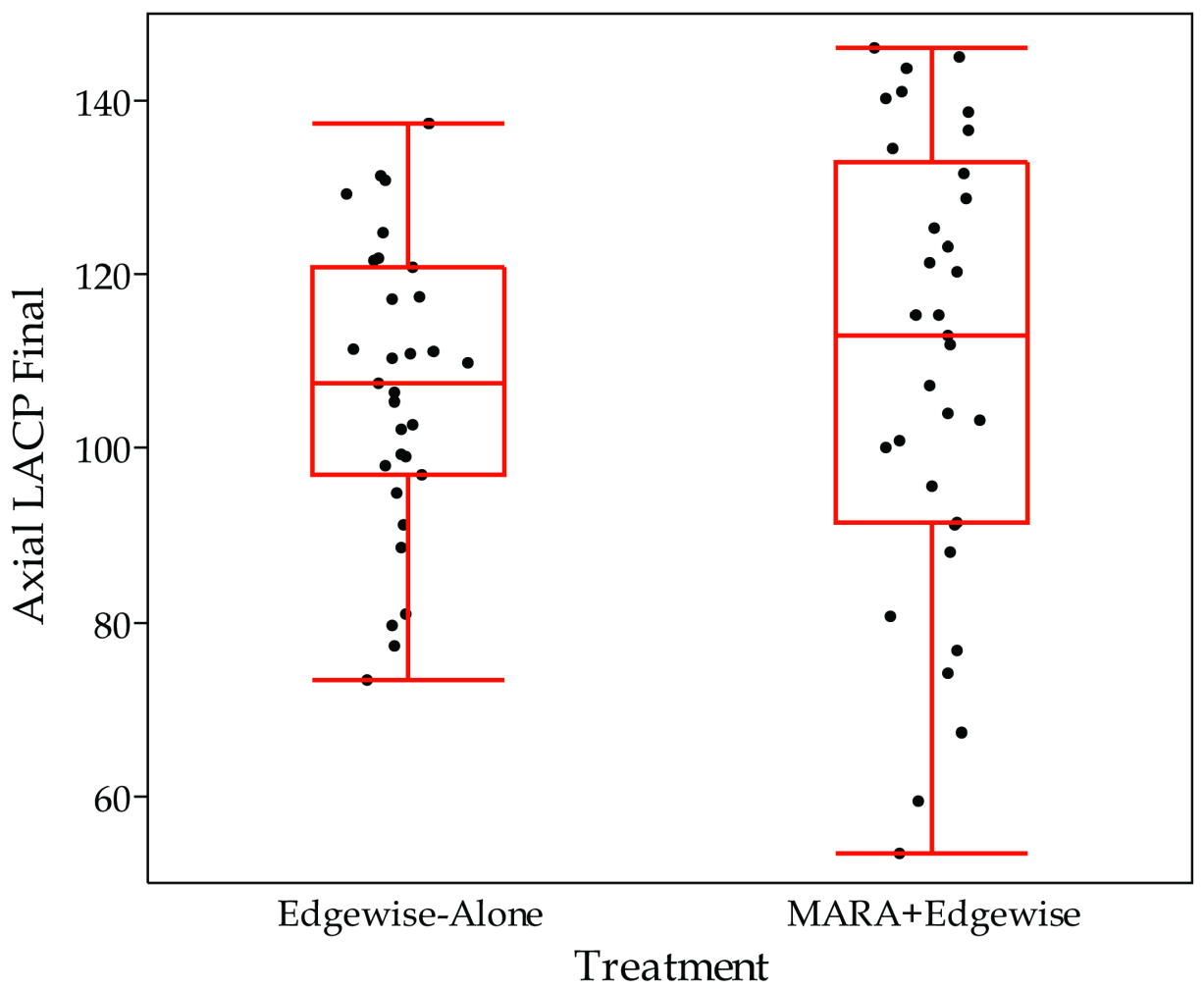

Figure 4-13. Box plots of Axial LACP Final, by treatment. 


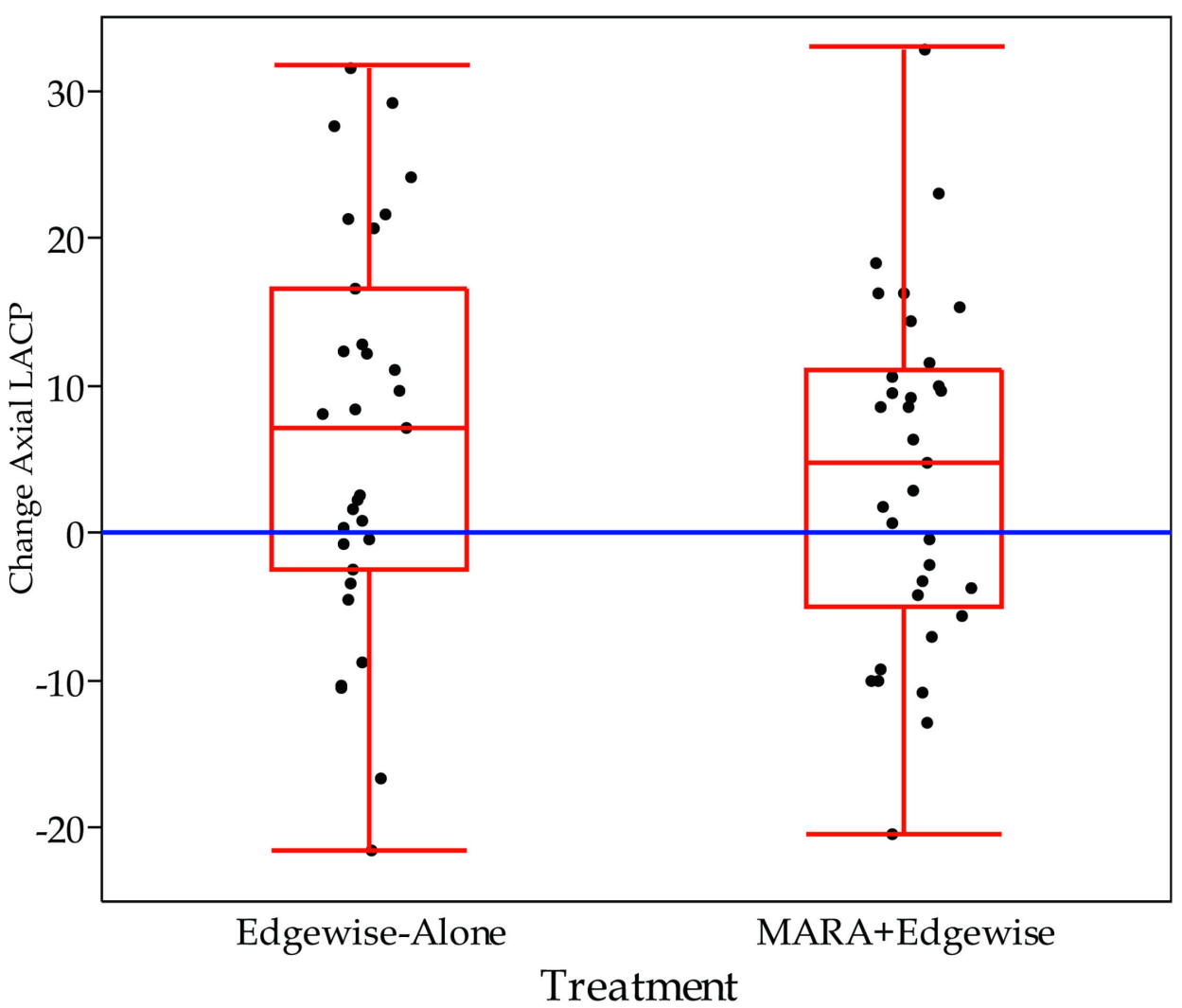

Figure 4-14. Box plots of the in-treatment change in Axial LACP, by treatment. The treatment change was larger in the Edgewise-Alone sample. 


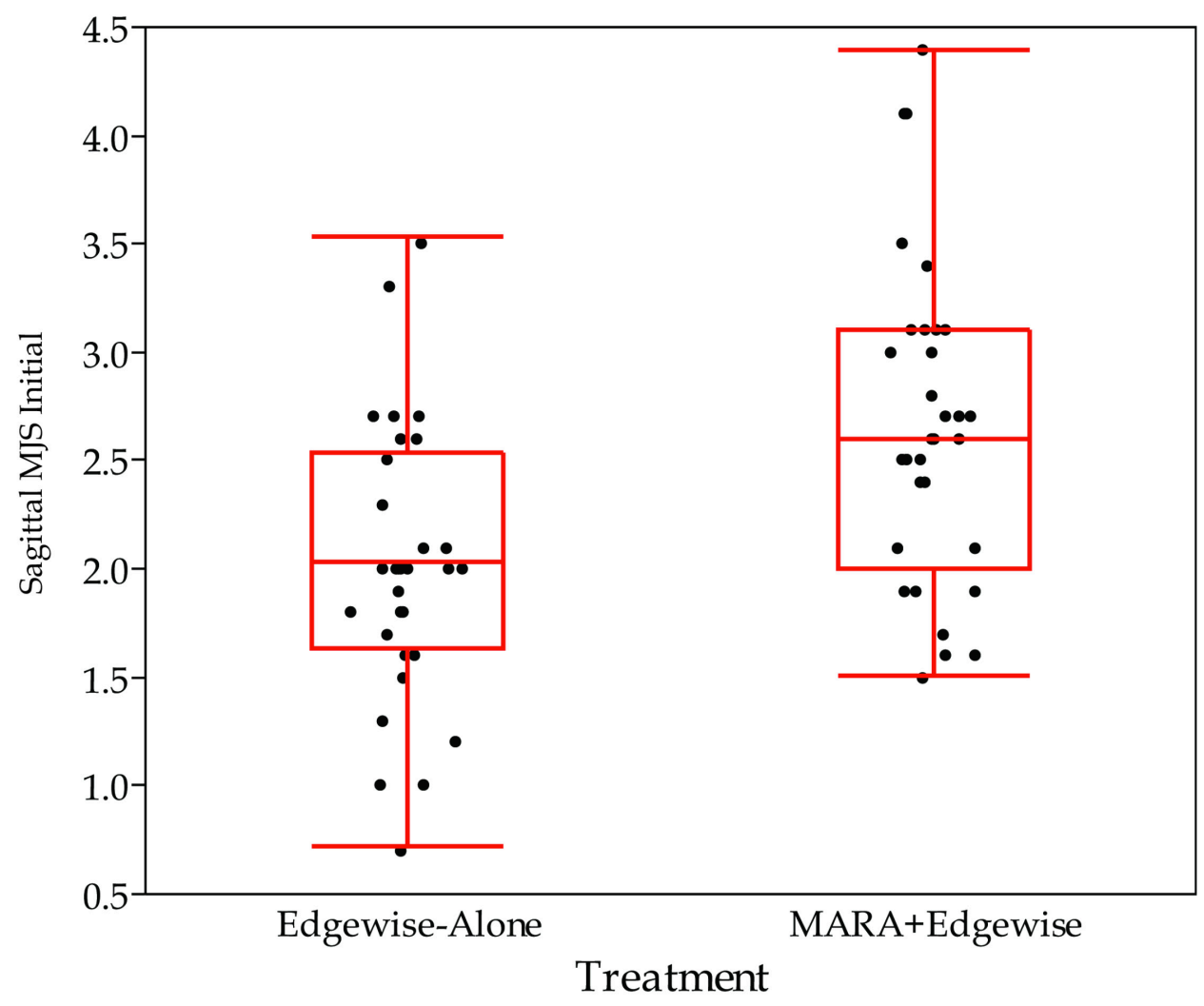

Figure 4-15. Box plots of the Sagittal MJS dimension at the start of treatment.

This dimension was significantly longer in the MARA+Edgewise sample at the start of treatment. 


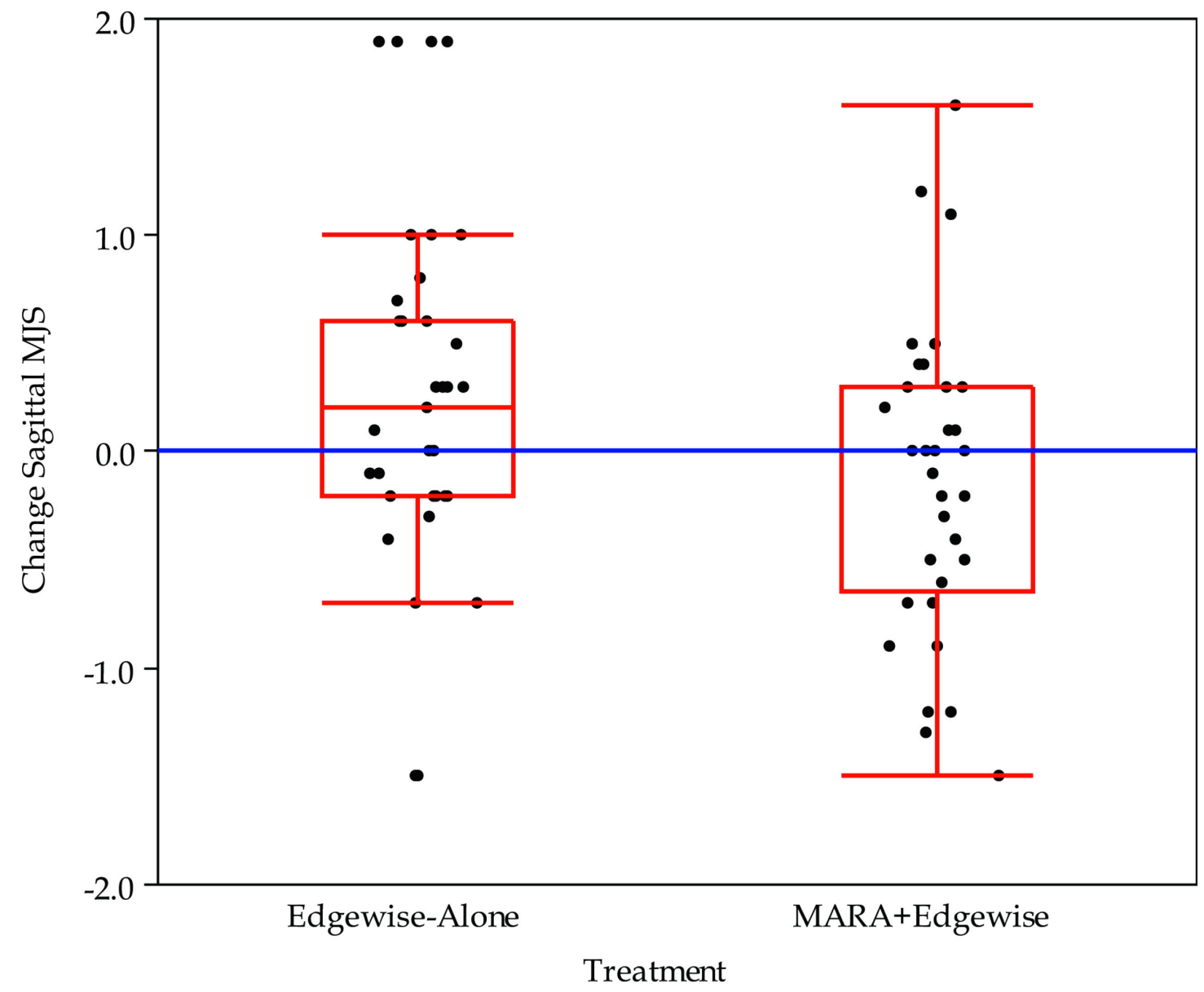

Figure 4-16. Box plots of the in-treatment changes in the Sagittal MJS dimension, by treatment.

Using these raw statistics, there was almost no average change in the MARA+Edgewise group, but a small but significant increase, averaging about $1 / 4 \mathrm{~mm}$ in the Edgewise-Alone sample. 


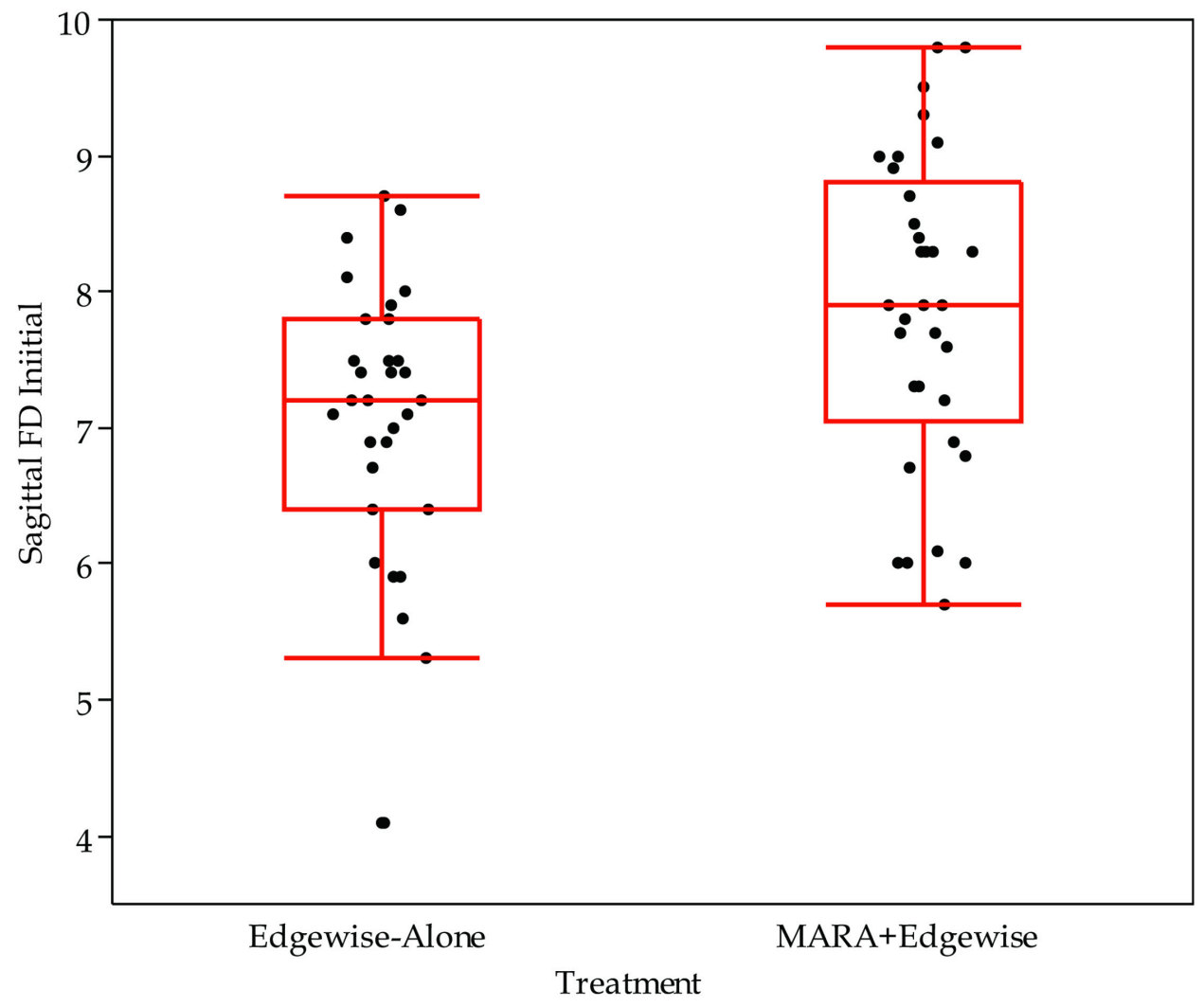

Figure 4-17. Box plots of the Sagittal FD dimension, by treatment.

Sagittal FD was significantly larger in the MARA+Edgewise sample at the start of treatment. 


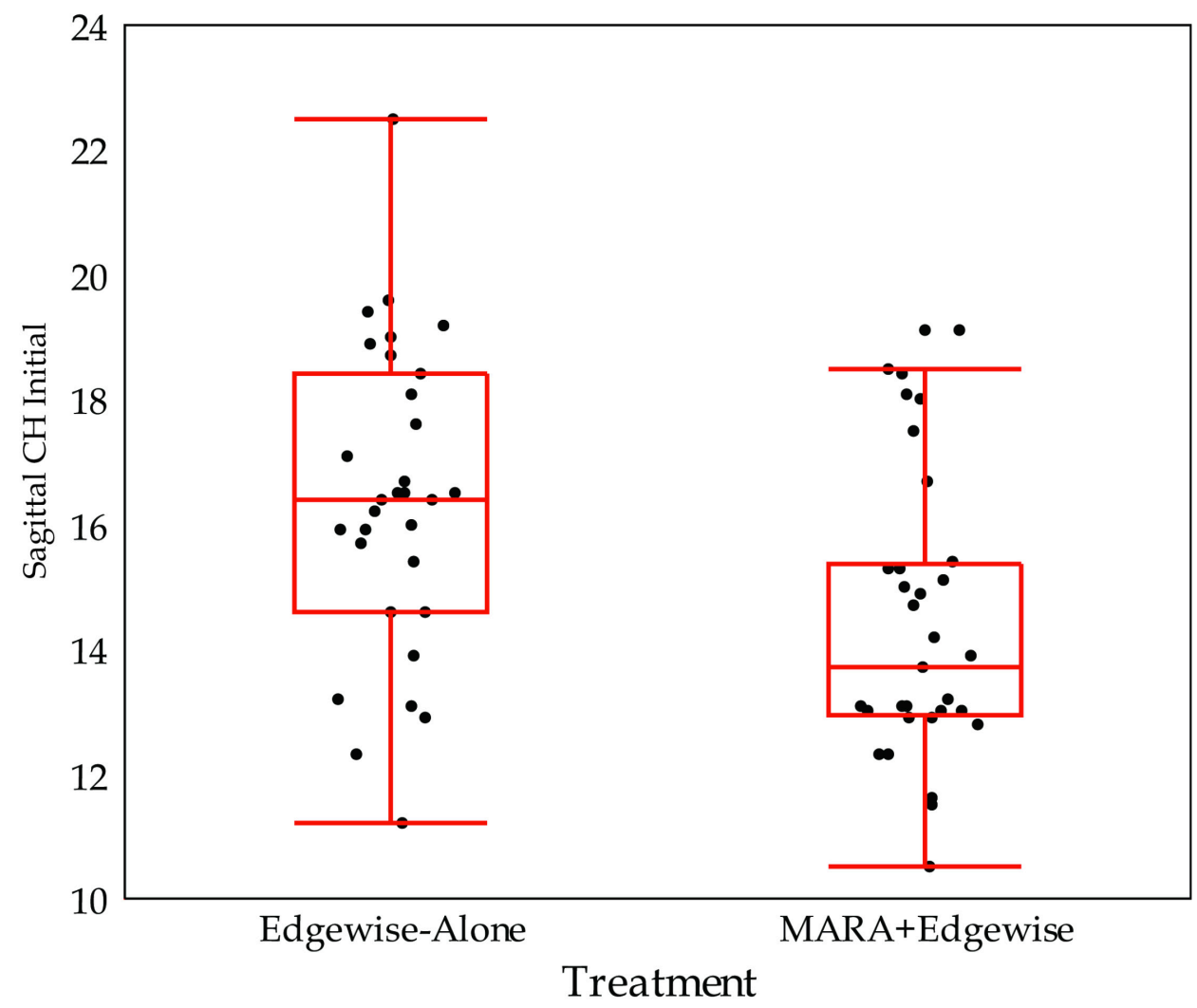

Figure 4-18. Box plots of the Sagittal CH dimension, by treatment.

Sagittal CH was significantly larger in the Edgewise-Alone sample at the start of treatment. 


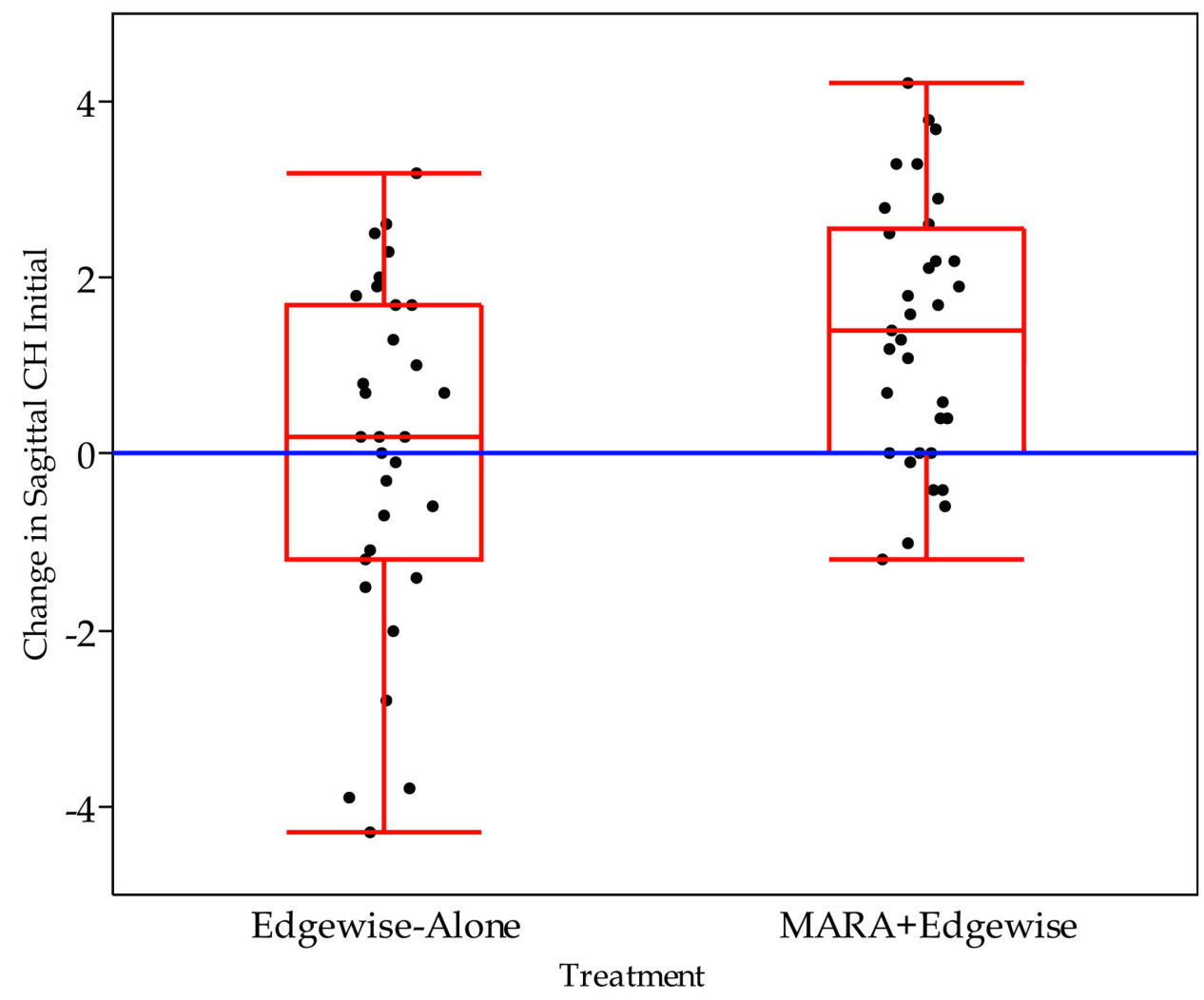

Figure 4-19. Box plots of the Sagittal in-treatment change in the $\mathrm{CH}$ dimension, by treatment.

Treatment increased this dimension significantly more in the MARA+Edgewise sample. The blue horizontal line is set at zero (no change). 


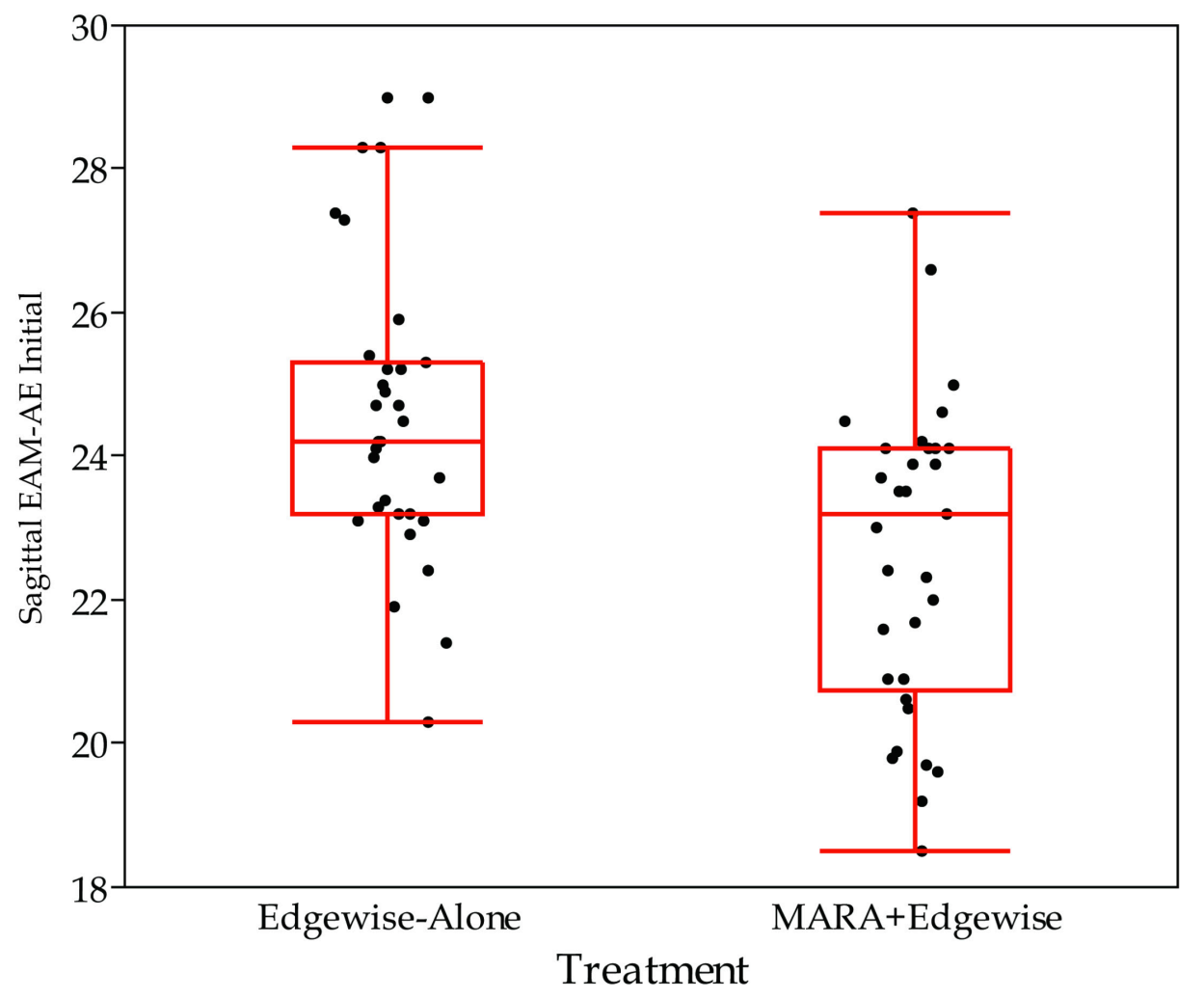

Figure 4-20. Box plots of the Sagittal EAM-AE dimension at the start of treatment.

This dimension was significantly larger in the Edgewise-Alone (Tennessee) sample at the start of treatment. 


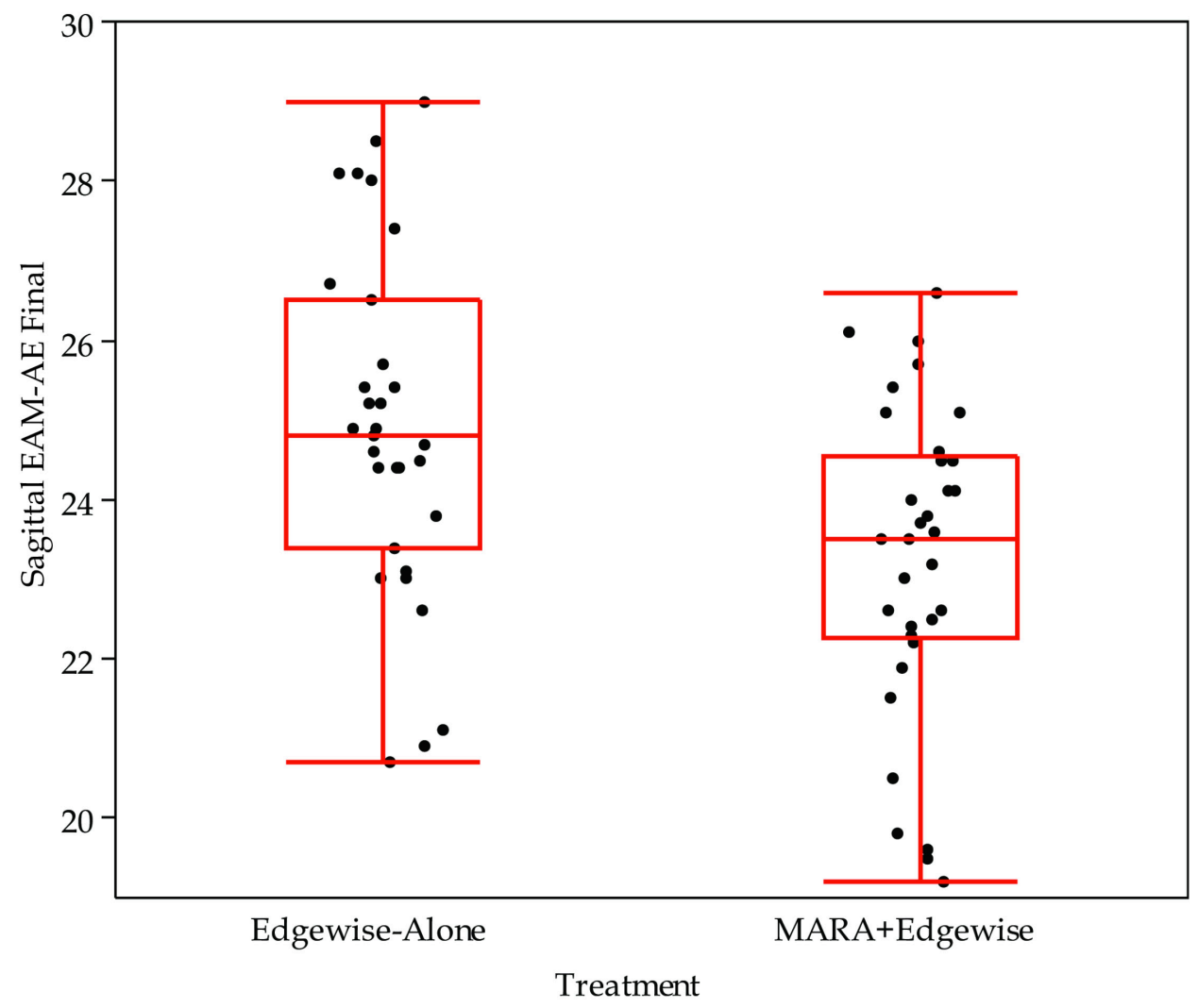

Figure 4-21. Box plots of the Sagittal EAM-AE dimension at the end of treatment.

This dimension was significantly larger in the Edgewise-Alone (Tennessee) sample at the end of treatment. 
statistically equivalent (not significantly different), however. This suggests that the MARA did not affect this dimension appreciably.

The next significant dimension, Sagittal ATA, is intriguing in that this dimension was significant between treatments both at the initial and final examinations, as well as the in-treatment changes. These results are graphed in Figures 4-22 through 4-25. This trait seemed to exhibit an intrinsic growth difference since it was larger at the start of treatment in the MARA+Edgewise group.

The next variable, sagittal area of the condylar head (ACP), was remarkable in that significant differences were found in the initial and final, but the in-treatment changes did not achieve significance (Figures 4-25 and 4-26). As was observed in evaluating the other measurements of the condylar head, the condylar head increased in the Edgewise alone sample and decreased slightly in the MARA+ Edgewise sample. This change almost achieved significance $(\mathrm{P}=0.055)$.

The last variable of note in regards to treatment differences was sagittal area of the glenoid fossa (AGF). In the initial data, the dimensions MARA + Edgewise group was significantly greater than the Edgewise alone group, which shows an inherent geographical difference in the sample groups (Figure 4-27). However, the Edgewise alone group changed more significantly in the area than the MARA + Edgewise group (Figure 4-28), which made the final comparisons insignificant. 


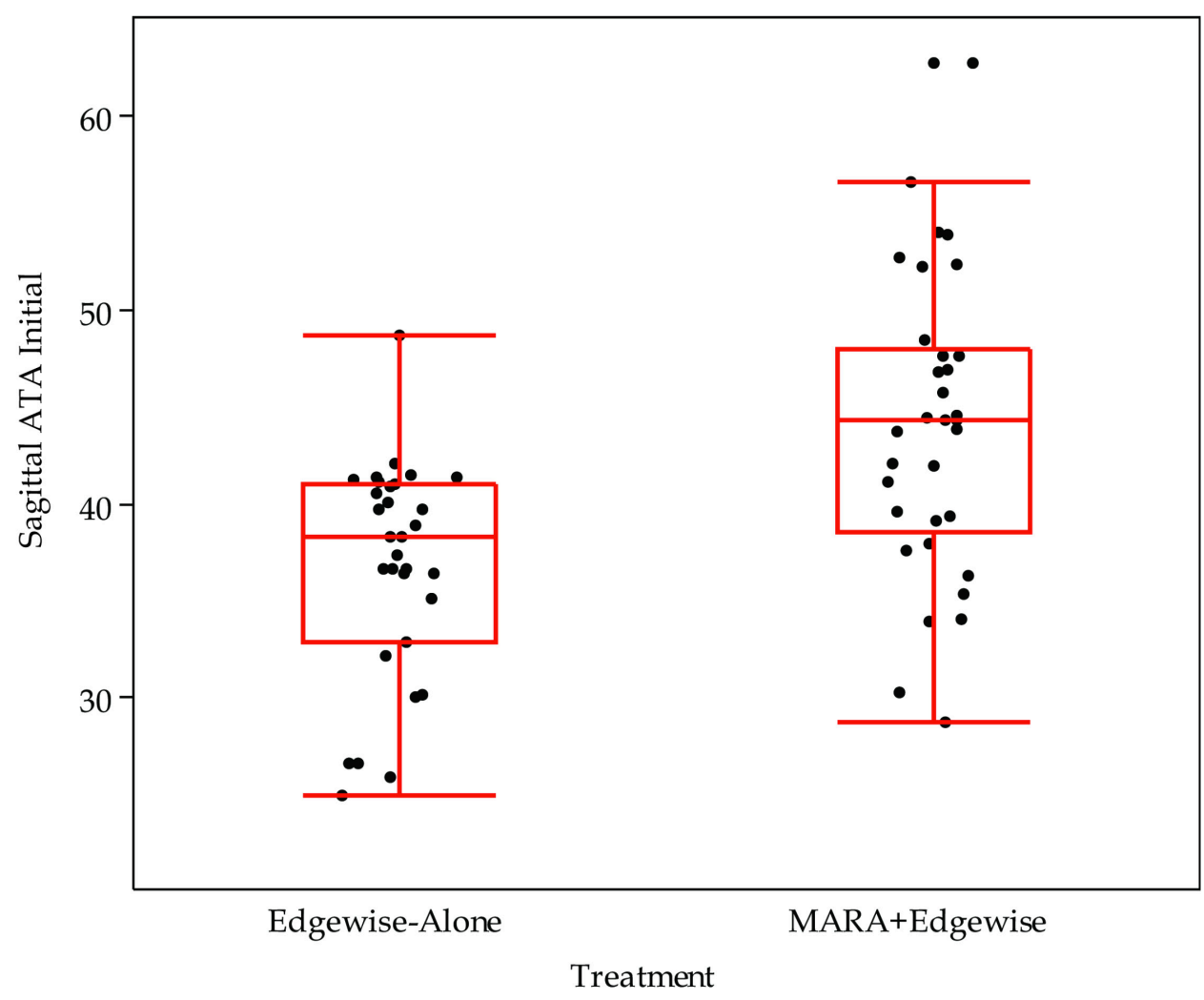

Figure 4-22. Box plots of the Sagittal ATA at the start of treatment.

This dimension was significantly larger in the MARA+Edgewise (Kansas) sample at the start of treatment. 


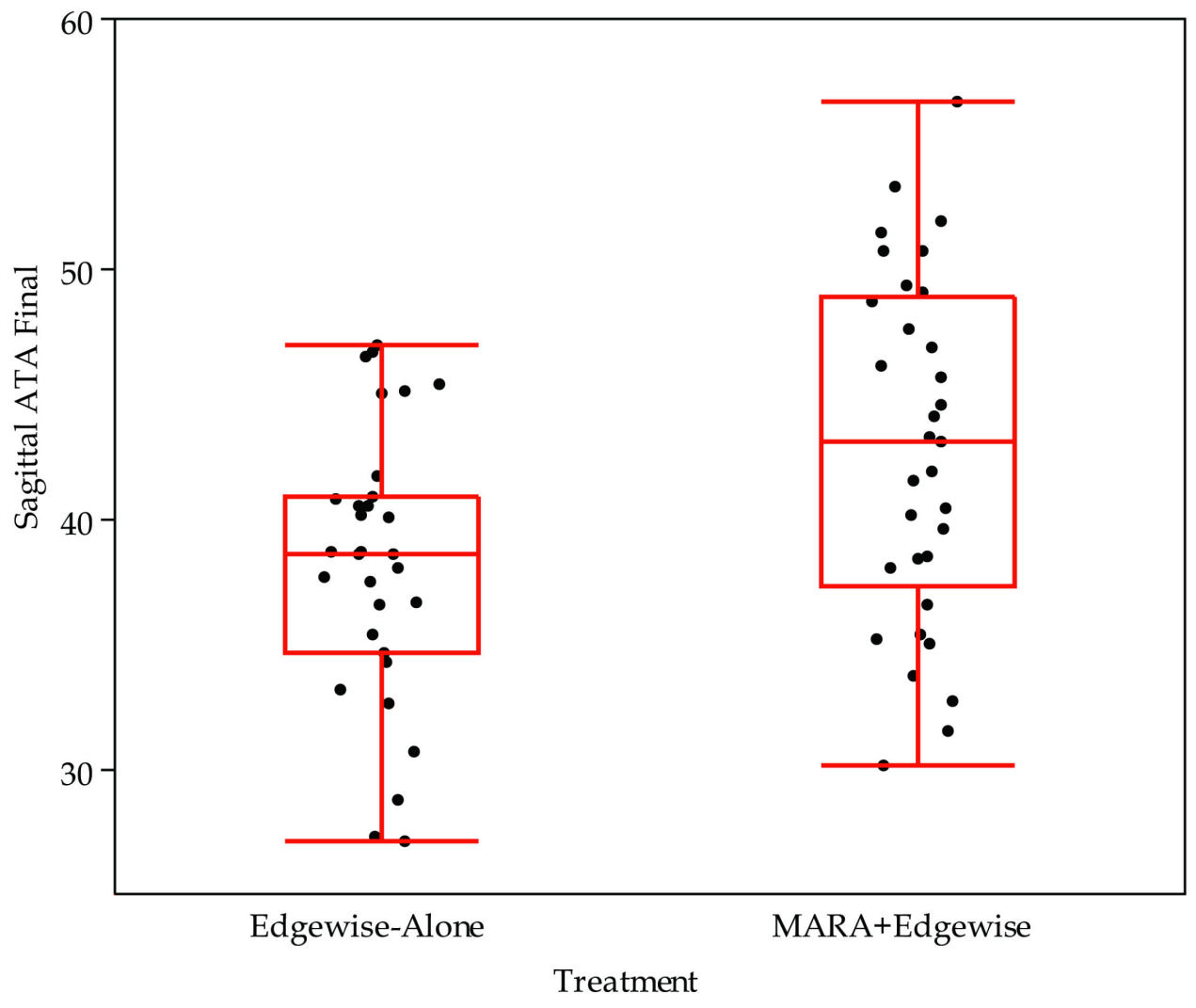

Figure 4-23. Box plots of the Sagittal ATA at the end of treatment.

This dimension was significantly larger in the MARA+Edgewise (Kansas) sample at both the start and end of orthodontic treatment. 


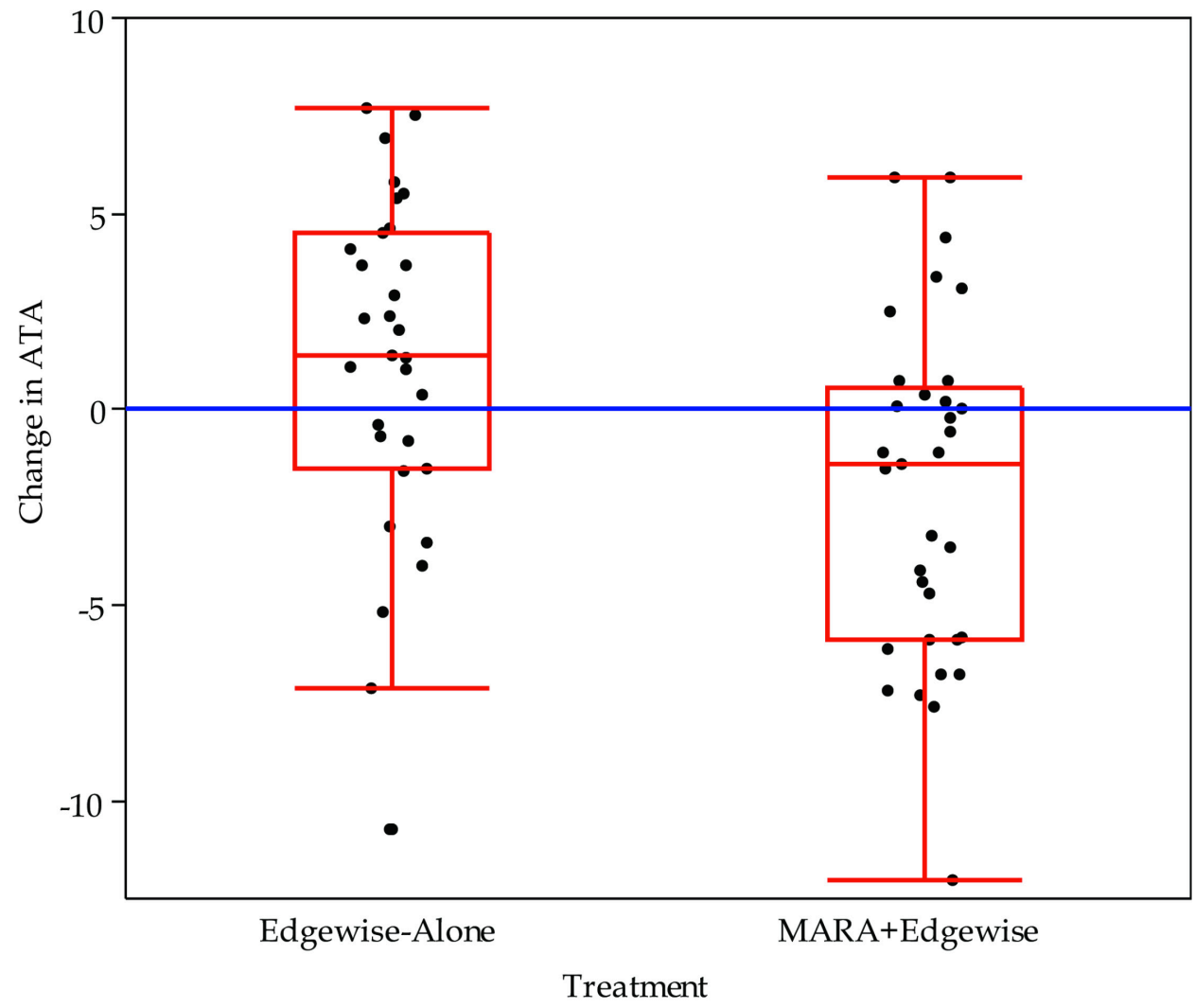

Figure 4-24. Box plots of the in-treatment changes in Sagittal ATA.

This dimension also was significantly larger in the MARA+Edgewise (Kansas) sample at the start and end of treatment. The blue horizontal line was set at zero (no change). 


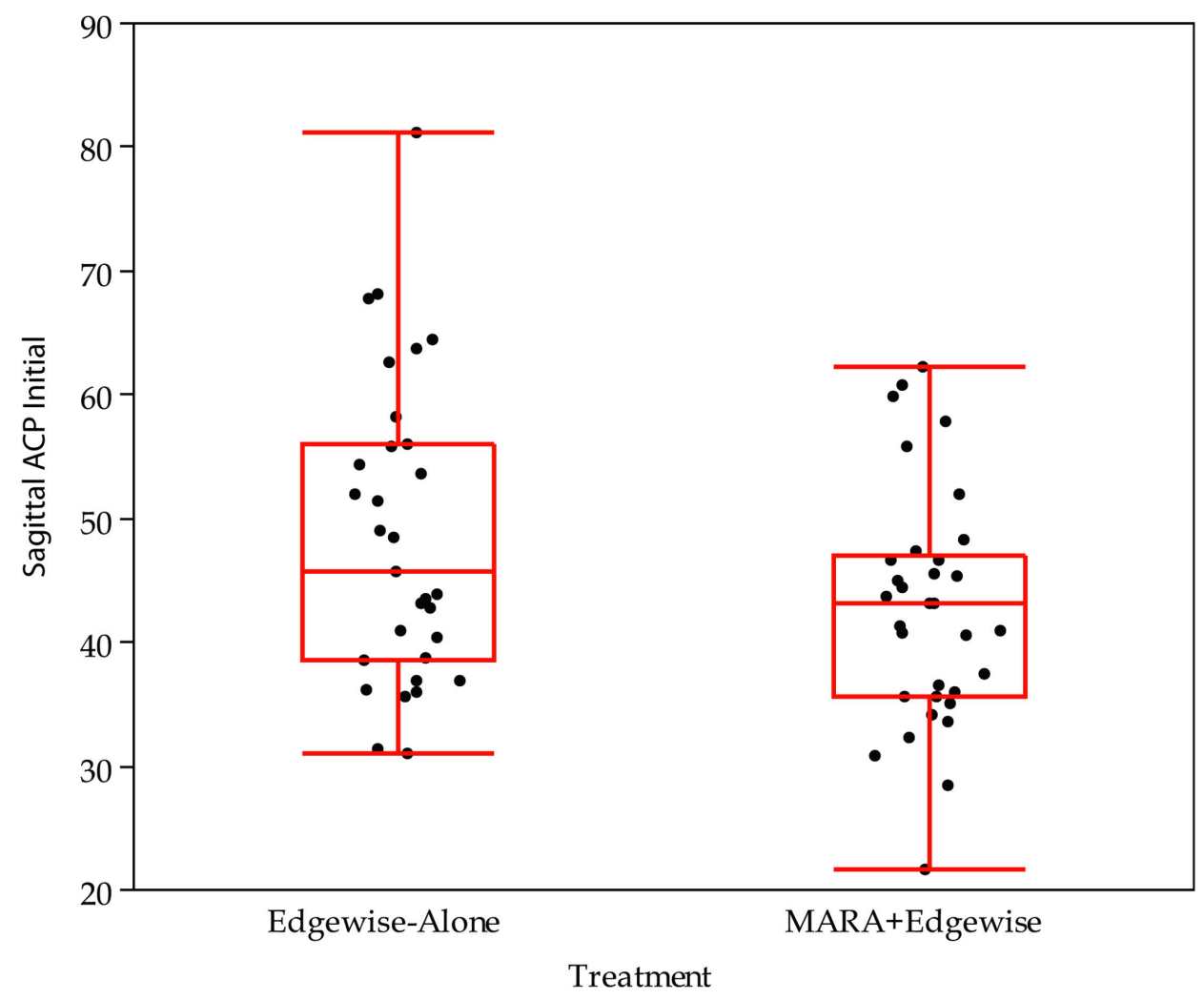

Figure 4-25. Box plots in Sagittal ACP at the start of treatment.

This dimension was significantly larger in the Edgewise-Alone (Tennessee) sample at the start and end of treatment. 


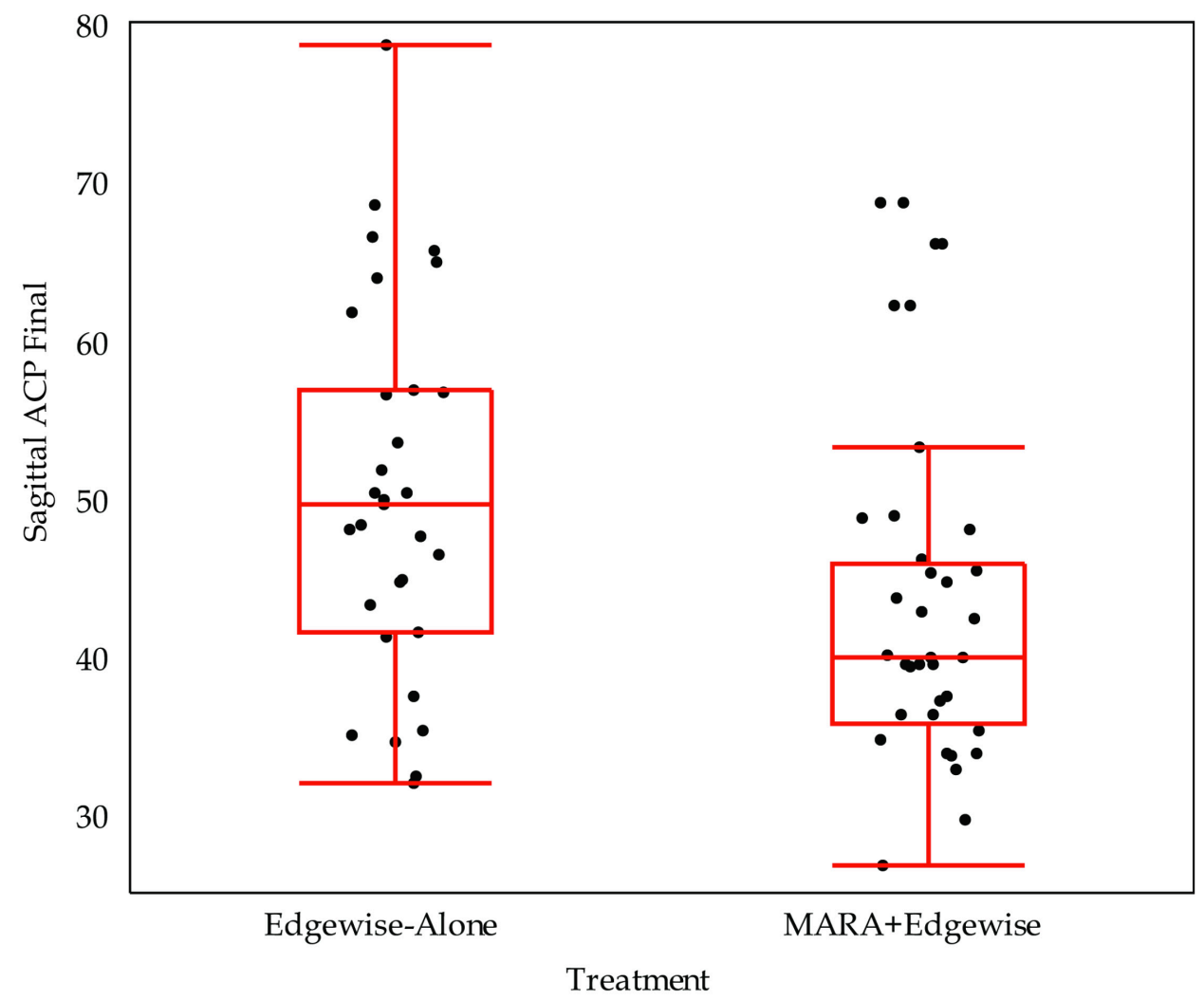

Figure 4-26. Box plots in Sagittal ACP at the end of treatment.

This dimension was significantly larger in the Edgewise-Alone (Tennessee) sample at the start and the end of treatment. 


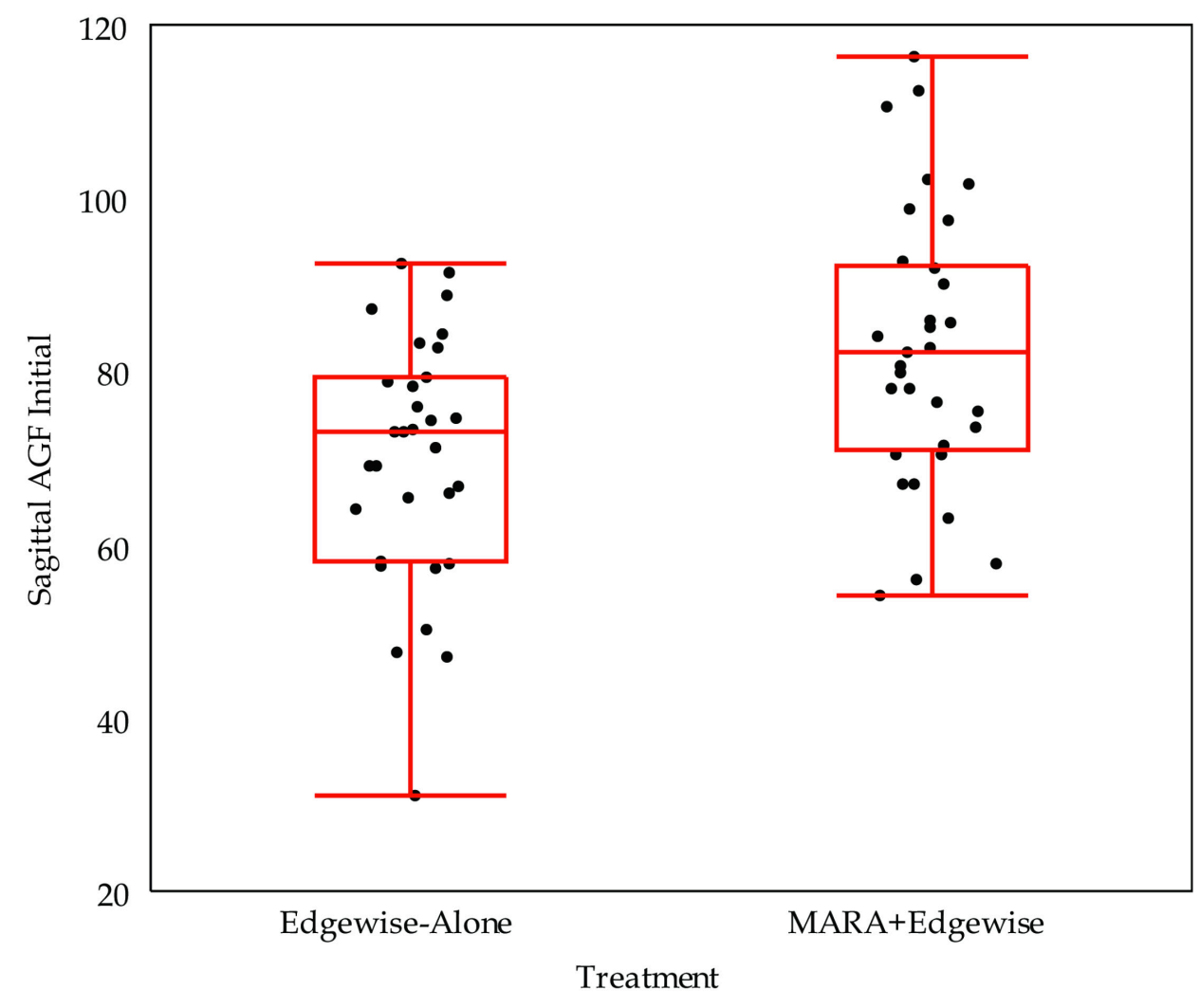

Figure 4-27. Box plots in Sagittal AGF at the start of treatment. 


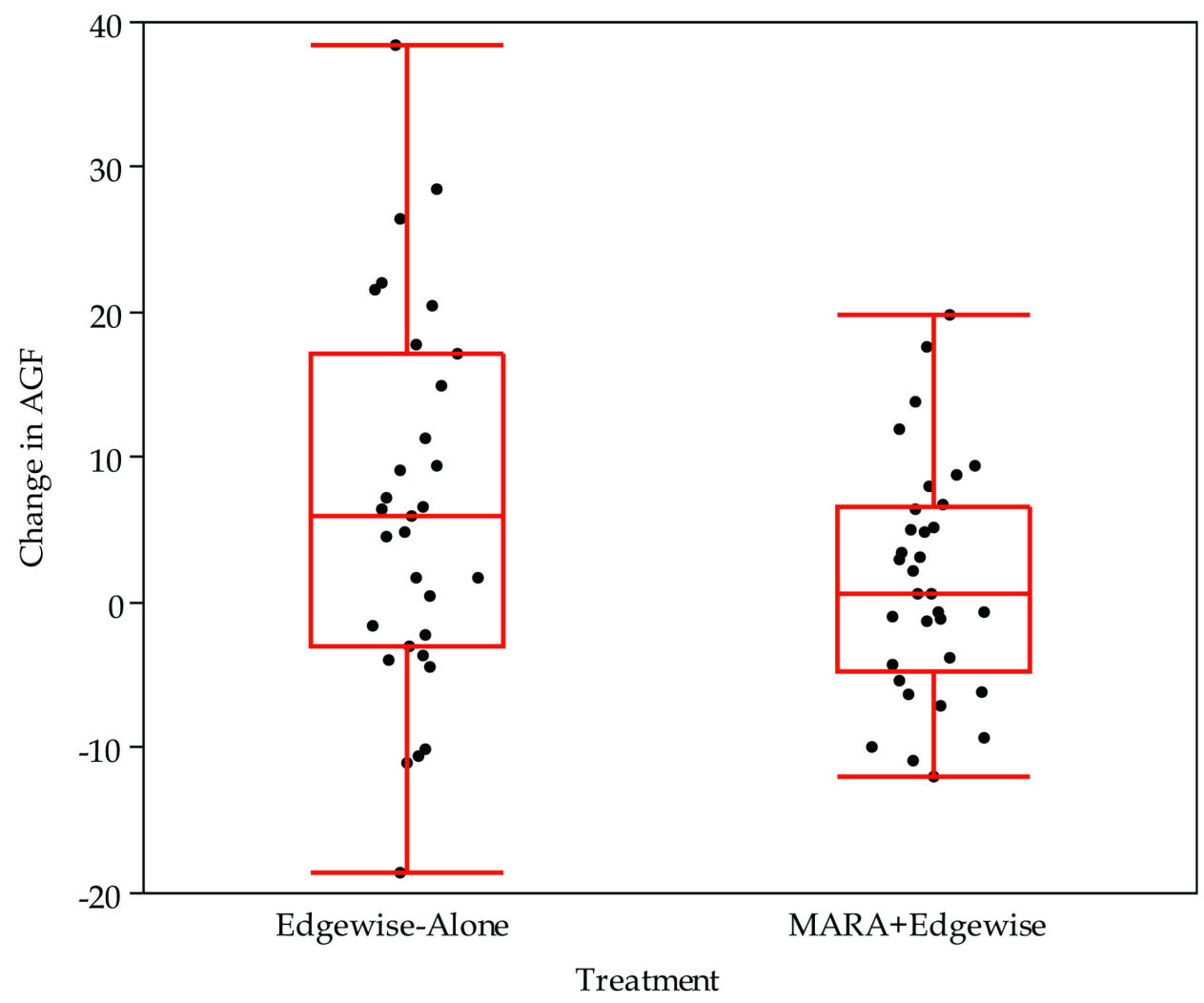

Figure 4-28. Box plots of the in-treatment changes in Sagittal AGF.

The change was significantly larger in the Edgewise-Alone group. 


\section{CHAPTER 5. DISCUSSION}

An ongoing argument is presently apparent in orthodontics about the amount of condyle-fossa remodeling and condylar growth seen in patients using functional appliance therapy in Class II skeletal patients. Some clinicians and researchers believe that functional appliances such as the MARA increase the amount of condylar growth and subsequent Class II skeletal correction, others believe that the mandible has a preset amount of growth and that conventional orthodontics will result in a similar amount of condylar growth to functional appliance therapy. This study reintroduced this dispute by comparing condylar remodeling in functional appliance therapy to conventional fixed orthodontic therapy Class II elastics. CBCT analysis was used in order to incorporate more accurate imaging than conventional radiography used in the past. We evaluated many changes induced by the MARA and conventional treatment, and compared the two groups to find any significant differences. This groundbreaking study showed significant results that will give clinicians an understanding of the changes in the TMJ during orthodontic treatment.

\section{Operator Reliability (Bias)}

In a "hot-topic" study such as this, the accuracy of the study is paramount in order to substantiate the claims. The use of functional appliances has spanned over 3 decades, and their perceived efficacy to correct skeletal and dental Class II malocclusions has differed between clinicians (Wahl 2006). During the study, many precautions were taken in order to validate the data accrued from the samples.

First, we derived the subjects in the study by evaluating consecutively treated patients from the patient archives of both practices (TN and KS). Certain parameters has to be met in order to qualify for inclusion in the study: Caucasian children aged 9 to 14 , ANB of greater than 3.5, overjet greater than 5, no significant transverse dental issues, and no dental developmental issues. Once the samples were collected, a two-way factorial ANOVA model was used on the seven cephalometric variables to calculate the similarity of the groups based on treatment and sex (Table 4-1). It is of note that none of the tests was significant, so it was assumed that the treatment groups were very similar before any orthodontic treatment intervention.

After finding the total sample, the subjects were randomized by an independent evaluator. A separate evaluator (author) then collected all of the 3D data. This randomization of the subjects before data collection removed any evaluation bias. Once the data was collected, intraoperator reliability was performed on the entire sample two months later. The 3D measurements were not saved on the images as to remove any previous methods of retrieving the data. Bland-Altman plots for the statistically significant variables were derived from the repeats, and only 8 of the 112 variables $(8 / 112=7.1 \%)$ were statistically significant at an unadjusted alpha level of 0.05 (Table 4-3). 


\section{Significant Differences}

The purpose of this study was to determine whether the MARA, a newer functional appliance, would cause statistically and clinically significant differences in the termporomandibular joint as compared to conventional orthodontic treatment. By evaluating the subjects with CBCT, we were able to evaluate many novel dimensions that were unavailable for previous studies. The variables $(\mathrm{k}=112$ dimensions $)$ assessed in this study were a combination of midline and paired dimensions. Analysis of paired (left, right) dimensions called for a more complex, mixed ANOVA model. With this, the repeated measure of "side" was evaluated, which measured the intra-subject changes, in addition to treatment and sex tests. The first step in interpreting this plethora of statistical results was scanning the 112 ANOVA models and collect the significant treatment effects $($ alpha $=0.05)$. The 25 significant F-ratios were listed in Table 4-4. These significant factors are discussed below.

\section{Significance by Treatment (Clinic)}

Although not one factor is weighed more than the rest, the significant differences in treatment by clinic was a potent factor is determining whether there is a difference between the functional and conventional groups. 19 of the 25 significant differences were due to geographics differences and treatment.

All of the subjects had Class II division 1 malocclusions, so the typical reduction seen in SNA was anticipated. Of interest significantly greater reduction was achieved in the Edgewise-Alone series $(-2.25$ degrees, $\mathrm{sd}=1.19)$. This may be a problem of having to treat the wrong jaw, however. Class II division 1 malocclusions most commonly present with mandibular retrognathia, which optimally would be treated by enhancing mandibular growth (if that were an option), not restricting maxillary growth. The MARA+Edgewise group did have a slight reduction in SNA (-0.81 degrees, $s d=1.31)$, but was not a great as the Edgewise alone group. These findings are similar to the amount of SNA change seen by Pangrazio-Kulbersh et al. 2003 in their MARA sample group $\left(-0.4^{\circ}\right)$.

The changes in the angle SNB (Figure 4-11) showed significant difference by treatment. In the Edgewise-Alone sample, the angle got smaller $(-0.46$ degrees, $s d=1.1)$, though the median was less than a degree. One would suspect that, with the increased correction of the Class II and growth, this dimension would increase as well. However, in using Class II elastics, there is a vertical component as well as a horizontal component. This could cause a clockwise rotation in the mandible, giving the perceived decrease in the horizontal component. In the MARA+Edgewise group, the median came forward a bit (0.52 degrees, $\mathrm{sd}=1.24)$. Pangrazio-Kulbersh et al. also found a forward relocation of $\mathrm{B}$ point in their MARA sample $\left(1.1^{\circ}\right)$, and they also found that the FMA decreased on average in their sample $\left(-0.1^{\circ}\right)(2003)$. Statistically, the difference between treatments in our study achieved significance $(\mathrm{P}=0.0031)$. Inspection of just this measure suggests 
that the MARA does aide in repositioning the mandible forward with possibly less of a vertical vector of force than conventional interarch elastics.

A significant difference in the posttreatment result of the maxillary-mandibular mesial molar relation was seen between the groups. The Edgewise (TN) group ended up at $-1.15 \mathrm{~mm}(\mathrm{sd}=1.11)$, which meant that the maxillary molar mesiobuccal cusp was distal to the mandibular mesiobuccal cusp indicating a Class I relationship. The MARA (KS) group posttreatment was $-0.31 \mathrm{~mm}(\mathrm{sd}=0.97)$, showing that the maxillary cusp was distal to the mandibular cusp; however, this difference was significant. The changes from pre- to posttreatment were not significant, indicating that there may have been a nonsignificant greater initial correction needed in the MARA group than the EdgewiseAlone group.

A variable that did not complement the rest of the data was cephalometric SellaVertical-to-M. This novel measurement was used to find the amount of horizontal advancement of the mandibular corpus as related to a relatively fixed structure, Sella. The Edgewise group surprisingly had more advancement in this measurement than the MARA (6.08 mm, $\mathrm{sd}=3.41$ Edgewise versus $2.58 \mathrm{~mm}$, $\mathrm{sd}=2.69$ MARA). This does not correspond to the changes seen in SNB and $\mathrm{CH}$, which would support more propulsion of the mandible in the MARA group.

In looking at the differences in the axial anteroposterior measurement of the condyle (Figure 4-12), there is a significant difference by treatment. In both the Edgewise-Alone and MARA+Edgewise group, the linear measurement decreased, but the difference between treatments achieved significance $(\mathrm{P}=0.0437)$. The Edgewise-Alone and MARA+Edgewise groups' mean changes were less than a millimeter $(-0.02 \mathrm{~mm}$ for Edge vs. $-0.36 \mathrm{~mm}$ for MARA). This shows more decreasing change of the condylar head in the MARA group versus the Edgewise-Alone group. Bell (1982) stated that moderate loading facilitates normal remodeling of the condyle, but if the demands for change are too rapid, degenerative remodeling may take place instead. Since the MARA repositions the condyles forward against the articular eminence and more resorption was seen in those subjects, the anterior resorption of the condylar head may have been due to higher compressive forces of the condyle against the eminence in the MARA+Edgewise group.

The next significant dimension, Axial LACP, is interesting in that this dimension was significant between treatments both at final examinations and the in-treatment changes while being similar at the initiation of treatment. The Edgewise-Alone group had and average change of $5 \mathrm{~mm}^{2}(\mathrm{sd}=13.75)$, while the MARA+Edgewise group had an average change of $4.25 \mathrm{~mm}^{2}(\mathrm{sd}=13.92)$. The average areas of the condyles posttreatment for the Edgewise-Alone and MARA groups were $107.7 \mathrm{~mm}^{2}(\mathrm{sd}=16.46)$ and $105.8 \mathrm{~mm}^{2}(\mathrm{sd}=24.69)$, respectively. These results are graphed in Figures 4-12 and 4-13. With the AP dimension decreasing more in the MARA+Edgewise group and similar increases in length seen in the axial ML dimension of the condyles, the assumption that the Edgewise alone sample would have a greater increase was validated with the data. 
The Sagittal MJS dimension was significantly larger in the MARA+Edgewise (Kansas) sample at the start of treatment (Figure 4-15). The Tennessee group had an initial measurement average of $2.1 \mathrm{~mm}(\mathrm{sd}=0.53)$, while the Kansas group had an initial average of $2.68 \mathrm{~mm}(\mathrm{sd}=0.71)$. Since no orthodontic work had been done at these initial records, this site difference would seem to be attributable solely to geographical (population) differences of the patient pools and/or to case selection. In addition to the start of treatment, Sagittal MJS also differed significantly in the amounts of in-treatment change (Figure 4-16). On average, the Edgewise (TN) group joint space increased $(0.27$ $\mathrm{mm}, \mathrm{sd}=0.66)$, while the MARA group slightly decreased $(-0.08 \mathrm{~mm}, \mathrm{sd}=0.76)$. This differs from the Vanlaecken et al. study, where they found no significant differences in joint space pre-and posttreatment (2006). Ruf and Pancherz (1998) also noted in their Herbst study that the condylar position in the fossa is relatively unaffected by functional appliance therapy. While the changes were significant, the posttreatment joint spaces were similar and nonsignificant between the 2 groups $(2.28 \mathrm{~mm}$ versus $2.6 \mathrm{~mm})$. This shows that, by initial case selection, more MARA subjects may have had an initial malocclusion may have "unseated" the condyle, and with treatment, the condyle changed to a more natural position in the fossa.

One of the most important variables in noting any clinical treatment differences between the groups was sagittal condyle height $(\mathrm{CH})$. The Sagittal $\mathrm{CH}$ dimension was larger in the Edgewise versus the MARA group at the start of treatment $(16.38 \mathrm{~mm}, \mathrm{sd}=$ 2.46 and $14.32 \mathrm{~mm}, \mathrm{sd}=2.41$, respectively), but the MARA group increased significantly more during treatment as compared to the Edgewise group, also in the MARA+Edgewise group (Figure 4-19). It cannot be determined whether the greater change was due to treatment instead of a population (Kansas versus Tennessee) difference in intrinsic growth patterns, since the initial average age of the MARA group was slightly younger than the Edgewise Alone group (11.93 versus 12.37). Nevertheless, it could be ascertained that there were geographical differences at the initiation of treatment, and that the MARA group had significantly greater growth of the condylar process $(1.45 \mathrm{~mm}$, sd $=1.77$ for MARA; $0.13 \mathrm{~mm}, \mathrm{sd}=1.5$ for Edgewise). These findings would associate with those studies that were considered proponents of the advantages of functional appliance therapy; however, the difference on average is less than $1.5 \mathrm{~mm}$, so the clinical significance may be minimal.

At both the start and end of treatment the dimension EAM-AE was larger in the Edgewise-Alone (Tennessee) sample. This is graphed for the start of treatment in Figure 4-20, and at the end of treatment in Figure 4-21. The amounts of in-treatment change were statistically equivalent (not significantly different), however. This suggests a geographical difference in the sample groups.

The next significant dimension, Sagittal ATA, is intriguing in that this dimension was significant between treatments both at the initial and final examinations, as well as the in-treatment changes. These results were graphed in Figures 4-22, 4-23, and 4-24. On average, the articular eminence of the MARA group (44.37 degrees, $\mathrm{sd}=6.28$ ) initially had a more obtuse angle than the Edgewise-Alone group (36.74 degrees, sd = $5.74)$, but the slope in the MARA group became more acute during treatment $(-2.07$ 
degrees, $s d=4.23)$ and the eminence in the Edgewise-Alone group became more obtuse (1.34 degrees, $s d=4.17$ ). No explanation can be reasoned for the changes in this variable other than the possible treatment differences causing different effects on the articular eminence.

The next considerable difference between the treatment groups was sagittal area of the condyle (ACP). Pretreatment, the MARA group was significantly smaller than the Edgewise-Alone group $\left(42.36 \mathrm{~mm}^{2}, \mathrm{sd}=9.29\right.$ and $48.97 \mathrm{~mm}^{2}, \mathrm{sd}=10.77$, respectively), while the posttreatment results showed a decrease in condylar size of the MARA group $\left(42.17 \mathrm{~mm}^{2}, \mathrm{sd}=9.43\right)$ and increase in the Edgewise-Alone group $\left(50.13 \mathrm{~mm}^{2}, \mathrm{sd}=\right.$ 11.97). While initial and final variables were significant, the average changes were not quite significant $\left(1.16 \mathrm{~mm}^{2}\right.$ versus $-0.35 \mathrm{~mm}^{2}, \mathrm{P}$ value $\left.=0.055\right)$. These finding coincide with the significant changes seen in axial AP length of the condyle and axial LACP - the condylar head, on average, was smaller in the MARA group possibly due to more resorption of the anterior part of the condyle.

The final significant variable between the groups was sagittal area of the glenoid fossa (AGF). Initially, the groups were significantly different $\left(74.11 \mathrm{~mm}^{2}, \mathrm{sd}=14.81\right.$ for Edgewise-Alone; $82.45 \mathrm{~mm}^{2}$, sd $=15.76$ for MARA), but the final treatment numbers were nonsignificant due to the significant changes in the dimension $\left(7.32 \mathrm{~mm}^{2}, \mathrm{sd}=\right.$ 12.75 for Edgewise-Alone; $0.5 \mathrm{~mm}^{2}, \mathrm{sd}=7.7$ for MARA). The final posttreatment numbers became more similar due to the significantly higher increase in glenoid fossa space in the Edgewise-Alone group. Ruf and Pancherz (1998) looked at MRIs and also found that more bone apposition was seen on the inferior part of the glenoid spine, causing a relative anteclination of the posterior border of the glenoid fossa. This, along with relative resorption of the anterior border of the glenoid fossa, could contribute to the relatively constant area of the glenoid fossa.

\section{Side-by-Treatment Significant Differences}

Another interesting type of difference found was the intra-individual differences between the left and right "sides" of the individual by treatment. Of the results, 4 variables were seen to be significant for side:

1. Initial sagittal fossa width,

2. Final sagittal fossa width,

3. Final sagittal EAM-AE, and

4. Initial sagittal AGF.

Fossa width was an interesting variable. Initially, the right fossa width was significantly bigger on average than the left in the Tennessee group $(18.07 \mathrm{~mm}$ and $17.48 \mathrm{~mm})$, while the left FW was bigger on average than the right in the Kansas group (17.2 mm and 16.73 
$\mathrm{mm}$ ). In the final measurements, the same circumstance held true for both groups, showing an intrinsic difference in fossa width size for the two treatment groups. The final measurements for Sagittal EAM-AE were also significant for side and treatment. The average measurement for the right side of the Tennessee sample (24.92 $\mathrm{mm}$ ) was significantly longer than the left side for Tennessee $(23.96 \mathrm{~mm})$, and both the left and right sides of the MARA group (23.78 $\mathrm{mm}$ and $23.23 \mathrm{~mm}$, respectively). The initial measurement of sagittal area of glenoid fossa (AGF) was noteworthy in that the left area of the Tennessee sample on average $\left(69.95 \mathrm{~mm}^{2}\right)$ was significantly smaller than the right side $(78.26 \mathrm{~mm})$ and both the left and right sides of the MARA sample $\left(82.42 \mathrm{~mm}^{2}\right.$ and $82.48 \mathrm{~mm}^{2}$, respectively). This initial measurement, as stated before, can be attributed to the intrinsic geographical differences of the sample.

\section{Treatment-by-Sex Differences}

Another remarkable factor was treatment-by-sex, or the differences seen in treatment categorized by gender. Two variables were significant for treatment by gender type: change in sagittal condyle head width (CHW) and final sagittal condylar head angle (CHA). For the final measurements in sagittal CHA, Tennessee boys had a more obtuse condylar head angle on average than girls (139.35 degrees vs. 132.06 degrees, respectively), while Kansas boys had a more acute angle on average than girls (131.48 degrees vs. 136.17 degrees, respectively). This may be due to treatment, geographical differences, or both; more studies may need to be done in order to confirm these changes and their degree of significance.

\section{Treatment Differences Seen in Both Groups}

Although only 25 significant variables were seen between both treatments, we prudently evaluated the posttreatment changes for all variables. Conveniently, we took descriptive statistics for the entire sample (Appendix A), so those variables that did not achieve inter-group statistical significance could be pooled together to see the average treatment changes for the entire sample of subjects. The following shows the average treatment changes for all of these variables and their significance level (alpha $=0.05)$.

\section{Cephalometric Variables}

Although the main focus of this study was to determine the temporomandibular changes during orthodontic treatment, an overview of treatment changes in the dental and skeletal relationships is important to evaluate in conjunction with the changes in the TMJs. These variables show overall treatment results.

Even though the changes in SNA and SNB were different by treatment, overall skeletal change in ANB was very similar between the groups. Average ANB decreased by -1.56 degrees for both groups (-1.79 degrees for Edgewise; -1.35 degrees for MARA). 
In terms of incisor position, overbite decreased (-2.34 mm Edgewise, -2.03 mm MARA; average $=-2.18 \mathrm{~mm})$ in both groups and overjet decreased $(-3.54 \mathrm{~mm}$ Edgewise, -2.68 mm MARA; average $=-3.11 \mathrm{~mm})$, with both of these measurements showing significant improvement. While the posttreatment numbers for mesial molar relationship were significantly different between both groups, significant improvement in molar relationship was similar for both groups $(-2.47 \mathrm{~mm}$ Edgewise, $-1.78 \mathrm{~mm}$ MARA; average $=-2.1 \mathrm{~mm}$ ). Similar changes were seen in the Pangrazio-Kulbersh et al. study for the MARA treatment group (-1.4 ${ }^{\circ}$ ANB) (2003).

In evaluating these variables, an overall correction in the Class II malocclusion was seen for both groups. Dentally, overbite, overjet, and the molar relationship improved on average in all patients. Skeletally, the ANB decreased in both groups, although the underlying jaw correction was different for both groups.

\section{Axial Variables}

The diagnosis of the axial dimension of the TMJs is remarkable in that this plane could not be measured before with conventional radiography. Some interesting changes were seen in this dimension. In regards to the mediolateral length of each condyle, both groups had an average increase of $0.815 \mathrm{~mm}(0.81 \mathrm{~mm} \mathrm{~L}$ condyle, $0.82 \mathrm{R}$ condyle). This change could be attributed to the innate growth seen in children this age. In terms of the distance of each condyle to the midsagittal plane (MSP), the L and R condyles in each group significantly widened further from the midline by increasing $1.4 \mathrm{~mm}$ on each side; this may be part of an innate lateral growth of the jaws and face. Condylar asymmetry, as evaluated with change in anteroposterior condylar distance (APCD), improved in the Edgewise group (from $0.31 \mathrm{~mm}$ to $0.01 \mathrm{~mm}$ ), while the MARA group's asymmetry grew slightly worse (from $0.09 \mathrm{~mm}$ to $0.14 \mathrm{~mm}$ ). These results, however, did not achieve statistical significance. The next variable evaluated was condylar angulation change as related to the MSP. The condyles on average became more obtuse in both groups (1.15 degrees $\mathrm{L}, 0.42$ degrees $\mathrm{R}$, average $=0.79$ degrees), but this difference did not achieve statistical significance.

\section{Sagittal Variables}

The variables included in the sagittal dimension are of note because some other studies have shown findings in this dimension using axially corrected tomographs.

In terms of joint space, the amount of change was insignificant for both groups. Anterior joint space decreased slightly $(-0.1 \mathrm{~mm} \mathrm{~L}, 0.03 \mathrm{~mm} \mathrm{R}$; average $=-0.04 \mathrm{~mm})$, and posterior joint space decreased slightly as well ( $-0.12 \mathrm{~mm} \mathrm{~L}$ and $\mathrm{R})$. The decrease in joint space coincides with a Herbst study done by Croft et al. (1999), in which he looked at horizontally corrected tomographs. They found a relative decrease in posterior joint space after treatment $(-0.4 \mathrm{~mm})$. Change in middle joint space was significant between both groups, but as a whole, the change was not significant $(0.05 \mathrm{~mm} \mathrm{~L}, 0.13 \mathrm{~mm} \mathrm{R}$; 
average $0.09 \mathrm{~mm}$ ). These results would follow the conventional thought that the temporomandibular joint space stays relatively constant throughout treatment (Ruf and Pancherz 1998, Vanlaecken et al. 2006).

Glenoid fossa width increased $(0.25 \mathrm{~mm} \mathrm{~L}, 0.32 \mathrm{~mm}$ R; average $0.29 \mathrm{~mm})$; glenoid fossa depth increased as well $(0.29 \mathrm{~mm} \mathrm{~L}, 0.24 \mathrm{~mm} \mathrm{R}$; average $0.27 \mathrm{~mm})$. Fossa depth and width would be assumed to increase with the increasing size of the condylar apparatus with growth and treatment.

In measuring the condylar head, the width showed no appreciable change though it decreased (-0.03 $\mathrm{mm} \mathrm{L},-0.09 \mathrm{~mm} \mathrm{R}$; average $-0.06 \mathrm{~mm})$; this change is in line with the assumption that the head resorbed on average in the AP dimension. Condylar head height increased for both groups $(0.11 \mathrm{~mm} \mathrm{~L}, 0.31 \mathrm{~mm} \mathrm{R}$; average $0.21 \mathrm{~mm})$, which is coincident to the condyle process increasing linearly in treatment for both groups.

In terms of the relative positioning of the TMJ as related to the external auditory meatus (EAM), all variables increased linearly; with the EAM being a relatively stable marker (Ricketts 1950), this showed a relative "forward positioning" of each of the landmarks involved. The distance to the articular eminence increased significantly $(0.92$ $\mathrm{mm} \mathrm{L}, 0.52 \mathrm{~mm} \mathrm{R}$; average $=0.72 \mathrm{~mm})$. The center of the condyle $(0.56 \mathrm{~mm} \mathrm{~L}, 0.2 \mathrm{~mm}$ $\mathrm{R}$; average $0.38 \mathrm{~mm})$ and the posterior wall of the glenoid fossa $(0.41 \mathrm{~mm} \mathrm{~L}, 0.1 \mathrm{~mm} \mathrm{R}$; average $0.26 \mathrm{~mm}$ ) also came forward. In a study by Wigal et al. (2011), 22 subjects treated with the Herbst were evaluated during pre-treatment, after Herbst therapy, and after phase II treatment. They evaluated the TMJ using lateral cephalograms. The authors found, similarly to our study, significant anterior movement of the condyle and the anterior portion of the glenoid fossa through treatment. Another study by Wadhawan et al. (2008) measured the location of the condyle in the fossa as related to the EAM. They used MRI to evaluate 12 children, and, like our study, they found a forward positioning of the post-glenoid spine as related to the EAM.

Another interesting variable of note is the change in the condylar head angle as related to the condylar process. This angle became more acute in both groups $(-7.11$ degrees L, -5.71 degrees R; average -6.41 degrees), showing significant remodeling of the condylar head forward in both groups. The MARA group on average had about a degree more acute repositioning than the Edgewise alone group, but this change was not significant between treatment groups.

As mentioned before in the statistically significant consortium, change in area of the condylar head (ACP) closely neared significance between groups, but when the groups were pooled together, the size of the condyle increased nonsignificantly $(0.45$ $\mathrm{mm}^{2} \mathrm{~L}, 0.49 \mathrm{~mm}^{2} \mathrm{R}$; average $=0.47 \mathrm{~mm}^{2}$ ). Finally, when evaluating the change in area of the articular eminence, both groups had a significantly average increase in size $(10.45$ $\mathrm{mm}^{2} \mathrm{~L}, 8.23 \mathrm{~mm}^{2} \mathrm{R}$; average $=9.34 \mathrm{~mm}^{2}$ ). Even with possible changes of the posterior border of the articular eminence, the structure increased in size with growth. 


\section{Coronal Variables}

The coronal dimension, not unlike the axial dimension, is incredible in that evaluating this aspect has been unavailable with conventional radiographic techniques. Coronal joint spaces (medial, superior, and lateral), similarly to the sagittal dimension, all showed nonsignificant changes pre- and post-treatment. Anterior joint space increased slightly in the L joint $(0.12 \mathrm{~mm})$ and decreased slightly in the R joint $(-0.02 \mathrm{~mm})$, superior joint space increased slightly in the $\mathrm{L}$ joint $(0.03 \mathrm{~mm})$ and decreased slightly in the $\mathrm{R}$ joint $(-0.1 \mathrm{~mm})$, and the posterior joint space decreased slightly in the L joint $(-0.03$ $\mathrm{mm})$ and increased slightly in the $\mathrm{R}$ joint $(0.06 \mathrm{~mm})$. These findings would be consistent with the belief that the joint space seems to be relatively stable post-treatment. 


\section{CHAPTER 6. SUMMARY AND CONCLUSION}

Much concentration has been seen in the orthodontic literature regarding functional appliances, and the debate is ongoing as to their treatment efficacy for the growing Class II patient as compared to conventional mechanics. The purpose of the present study was to add information through the analysis of cone beam computed tomographs (CBCT) comparing the temporomandibular effects of the MARA+Edgewise treatment compared to edgewise treatment alone with intermaxillary elastics. The goal was to identify any differing changes in the TMJ obtained from the use of the MARA that would benefit Class II correction compared to conventional treatment alone. The author selected MARA-treated cases and Edgewise-only conventional cases based on the following criteria: 1) 9-14 years of age, 2) ANB greater than 4 degrees, 3) positive overjet of greater than $3.5 \mathrm{~mm}, 4)$ non-extraction therapy, and 5) no posterior transverse problems or craniofacial anomalies.

The MARA plus Edgewise group consisted of 33 subjects, and the EdgewiseAlone group consisted of 31 subjects. The cephalometric and CBCT analysis was performed with Dolphin Imaging $11{ }^{\circledR}$ to measure 98 separate variables. Major findings were:

- In terms of cephalometric changes, both types of mechanics resulted in improving the overall Class II division 1 skeletal and dental relationships. Yet, there was on average less retrusion of the maxilla and more advancement of the mandible to correct the Class II in the MARA versus the conventional treatment group.

- More resorption of the condylar head was seen anteroposteriorly in the MARA group versus conventional treatment, causing the condyles of the MARA subjects to decrease in size on average compared to the increase seen in the conventional edgewise group.

- Lengthening of the condyle was statistically greater in the MARA group than the conventional treatment group, although both increased on average.

- The slope of the posterior border of the articular eminence became more acute in the MARA group and more obtuse in the conventional edgewise group. Further studies may need to be developed to explain this phenomenon.

- On average, the condylar head angle decreased for both treatment groups, showing a forward remodeling of the condylar head.

- Temporomandibular joint spaces, as a whole, stayed relatively stable throughout both treatment groups.

- Overall, there was a relative increase and forward advancement of the temporomandibular apparatus in both treatment groups, with the consequence of this being due to treatment and growth of the subjects. 


\section{LIST OF REFERENCES}

Allen-Noble PS. Clinical management of MARA. Allesee Orthodontic Appliances. July 2005.

Altman DG, Bland JM. Measurement in medicine: the analysis of method comparison studies. Statistician 1983;32:307-17.

Athanasiou A. Orthodontic Cephalometry. London: Mosby-Wolfe; 1995.

Baumrind S. The road to three-dimensional imaging in orthodontics. Semin Orthod 2011;17:2-12.

Bell W. Clinical management of temporomandibular disorders. Chicago: Year Book Medical Publishers; 1982.

Bishara S. Functional appliances: a review. Am J Orthod Dentofacial Orthop 1989;95:250-8.

Bland JM, Altman DG. Statistical methods for assessing agreement between two methods of clinical measurement. Lancet 1986;i,307-10.

Bland JM, Altman DG. Measuring agreement in method comparison studies. Stat Methods Med Res 1999;8:135-60.

Bland JM, Altman DG. Applying the right statistics. Ultrasound Obstetrics Gynecol 2003;22:85-93.

Bland JM, Altman DG. Statistical notes: measurement error proportional to the mean. $\mathrm{Br}$ Med J 1996;313:106.

Bland JM, Altman DG. Statistical notes: measurement error. Br Med J 1996;313:744.

Carlsson G, Öberg T. Remodeling of the temporomandibular joint. In: Zarb G, Carlsson G, eds. Temporomandibular joint function and dysfunction. St. Louis: CV Mosby;1979.

Cevidanes L. Clinical outcomes of Fränkel appliance therapy associated with a counterpart analysis. Am J Orthod Dentofacial Orthop 2003;123:379-387.

Charlier JP, Petrovic A, Stutzmann J. Effects of mandibular hyperpropulsion on the prechondroblastic zone of rat condyle. Am J Orthod 1969;55:71-4.

Cho BH, Ahn YW, Jung YH. Comparison of mandibular morphology between patients with temporomandibular joint osteoarthritis and asymptomatic normal subjects. Quintessence Int. 2009;40:e49-54. 
Clark W. Twin block functional therapy: applications in dentofacial orthopaedics. London: CV Mosby; 1997.

Cozza P, Baccetti T, Franchi L, Toffol LD, McNamara JA Jr. Mandibular changes produced by functional appliances in Class II malocclusion: a systematic review. Am J Orthod Dentofacial Orthop 2006;129:599.e1-12.

Creekmore TD, Radney LJ. Frankel appliance therapy: orthopedic or orthodontic? Am J Orthod 1983;83:89-108.

Croft RS, Buschang PH, English JD, Meyer R. A cephalometric and tomographic evaluation of Herbst treatment in the mixed dentition. Am J Orthod Dentofacial Orthop 1999;116:435-43.

Dahlberg G. Statistical methods for medical and biological students. London: George Allen and Unwin, 1940.

Degroote C. Alterability of mandibular condylar growth in the young rat and its implications. Leuven, 1984, Katholieke Universiteit Leuven.

Durkin J. Secondary cartilage: a misnomer? Am J Orthod 1972;62:15.

Durkin J, Heely J, Irving J. The cartilage of the mandibular condyle. Oral Sci Rev 1973;2:29.

Gianelly A, Bronson P, Martigononi M, Bernstein L. Mandibular growth, condylar position, and Frankel appliance therapy. Angle Orthod 1983;53:131-42.

Graber TM. Functional appliances. In: Graber TM, Vanarsdall RL, Vig KWL, editors. Orthodontics: current principles and techniques. St. Louis: CV Mosby; 2005. p. 493-542.

Graber TM, Rakosi T, Petrovic A. Dentofacial orthopedics with functional appliances. St. Louis: CV Mosby; 1997.

Hansson T. Thickness of the soft tissue and the articular disc in the temporomandibular joint. Acta Odontol Scand 1977;35:77.

Harris DB, Enlow DH. A study of the postnatal growth of the mandible. Am J Orthod 1964;50:25-50.

Harris EF, Smith RN. Accounting for measurement error: A critical but often overlooked process. Arch Oral Biol 2009; 54, Supplement 1:107-17.

Hilgers ML, Scarfe WC, Scheetz JP, Farman AG. Accuracy of linear temporomandibular joint measurements with cone beam computed tomography and digital cephalometric radiography. Am J Orthod Dentofacial Orthop 2005;128:803-11. 
Honey OB, Scarfe CW, Hilgers M, Klueber K, Silveira AM, Haskell BS, Farman AG. Accuracy of cone-beam computed tomography imaging of the temporomandibular joint: Comparisons with panoramic radiology and linear tomography. Am J Orthod Dentofacial Orthop 2007;132:429-38.

Hotz R. Application and appliance manipulation of functional forces. Am J Orthod 1970;58:459-78.

Houston WJB. The analysis of errors in orthodontic measurements. Am J Orthod 1983;5:382-90.

Houston WJ, Maher RE, McElroy D, Sherriff M. Sources of error in measurements from cephalometric radiographs. Eur J Orthod 1986;8:149-51.

Ikeda K, Kawamura A. Assessment of optimal condylar position with limited cone-beam computed tomography. Am J Orthod Dentofacial Orthop. 2009;135:495-501.

Johnston LE. Growth and the Class II patient: rendering unto caesar. Sem Orthod 1998;4:59-62.

Kang MG, Yun KI, Kim CH, Park JU. Postoperative condylar position by sagittal split ramus osteotomy with and without bone graft. J Oral Maxillofac Surg 2010;68:2058-64.

Knapp TR. 1992. Technical error of measurement: a methodological critique. Am J Phys Anthropol 87:235-236.

McNamara JA Jr., Brudon WL. Orthodontic and orthopedic treatment in the mixed dentition. Needham Press; 1993.

Meng F, Liu Y, Hu K, Zhao Y, Kong L, Zhou S. A comparative study of the skeletal morphology of the temporomandibular joint of children and adults. J Postgrad Med 2008;54:191-4.

Midtgård J, Björk G, Linder-Aronson S. Reproducibility of cephalometric landmarks and errors of measurements of cephalometric cranial distances. Angle Orthod 1974;44:56-61.

Öberg T, Carlsson G. Macroscopic and microscopic anatomy of the temporomandibular joint. In: Zarb G, Carlsson G, eds. Temporomandibular joint function and dysfunction. St. Louis: CV Mosby; 1979.

Okeson J. Management of temporomandibular disorders and occlusion. St. Louis: CV Mosby; 2007.

Pancherz H. Treatment of Class II malocclusions by jumping the bite with the Herbst appliance: a cephalometric investigation. Am J Orthod 1979;76:423. 
Pangrazio-Kulbersh V, Berger JL, Chermak DS, Kaczynski R, Simon ES, Haerian A. Treatment effects of the mandibular anterior repositioning appliance on patients with Class II malocclusion. Am J Orthod Dentofacial Orthop 2003;123:286-95.

Petrovic A, Stutzmann J, Oudet C. Control processes in the postnatal growth of the condylar cartilage of the mandible. In: McNamara J. Determinants of mandibular form and growth. Monograph No. 5, Caniofacial growth series, Center for Human Growth and Development, Ann Arbor, University of Michigan Press; 1975.

Popowich K, Major PW. Effect of Herbst treatment on temporomandibular joint morphology: a systematic literature review. Am J Orthod Dentofacial Orthop 2003;123:388-94.

Rees L. The structure and function of the mandibular joint. Brit Dent J 1954;96:125.

Ricketts RM. Variations of the temporomandibular joint as revealed by cephalometric laminography. Am J Orthod 1950;36;877-97.

Rodrigues AF, Fraga MR, Vitral RW. Computed tomography evaluation of the temporomandibular joint in Class II Division 1 and Class III malocclusion patients: condylar symmetry and condyle-fossa relationship. Am J Orthod Dentofacial Orthop 2009;136:199-206.

Rudzki-Janson I, Noachtar R. Functional appliance therapy with the Bionator. Semin Orthod 1998;4:33-45.

Ruf S, Pancherz H. Temporomandibular joint growth adaptation in Herbst treatment: a prospective magnetic resonance imaging and cephalometric roentgenographic study. Eur J Orthod 1998; 20:375-88.

Ruf S, Pancherz H. The mechanism of class II correction during Herbst therapy in relation to the vertical jaw base relationship. Angle Orthod 1997;67:271.

Sicher H. Oral Anatomy. St. Louis: CV Mosby; 1949.

VanLaeken R, Martin CA, Dischinger T, Razmus, T, Ngan P. Treatment effects of the Edgewise Herbst appliance: a cephalometric and tomographic investigation. Am J Orthod Dentofacial Orthop 2006;130:582-93.

Vieira S, Corrente JE. Statistical methods for assessing agreement between double readings of clinical measurements. J Appl Oral Sci 2011;19:488-92.

Voudouris JC, Kuftinec MM. Improved clinical use of Twin-block and Herbst as a result of radiating viscoelastic tissue forces on the condyle and fossa in treatment and long-term retention: growth relativity. Am J Orthod Dentofacial Orthop 2000;117:247-66. 
Voudouris JC, Woodside DG, Altuna G, Kuftinec MM, Angelopoulos G, Bourque PJ. Condyle-fossa modifications and muscle interactions during Herbst treatment, part 1. New technological methods. Am J Orthod Dentofacial Orthop. 2003a;123:604-13.

Voudouris JC, Woodside DG, Altuna G, Angelopoulos G, Bourque PJ, Lacouture CY, Kuftinec MM. Condyle-fossa modifications and muscle interactions during Herbst treatment, part 2. Results and conclusions. Am J Orthod Dentofacial Orthop. 2003b;124:13-29.

Wadhawan N, Kumar S, Kharbanda OP, Duggal R, Sharma R. Temporomandibular joint adaptations following two-phase therapy: an MRI study. Orthod Craniofac Res. 2008 Nov; 11:235-50.

Wahl N. Orthodontics in 3 millenia: Functional appliances to midcentury. Am J Orthod Dentofacial Orthop 2006;129:829-33.

Wigal TG, Dischinger T, Martin C, Razmus T, Gunel E, Ngan P. Condyle/Glenoid fossa changes of Class II patients treated with edgewise crowned Herbst appliance in the early mixed dentition period. Hong Kong Dent J 2011;8:9-17.

Woodside DG, Metaxas A, Altuna G. The influence of functional appliance therapy on glenoid fossa remodeling. Am J Orthod Dentofacial Orthop. 1987;92:181-9. 


\section{APPENDIX A. DESCRPTIVE STATISTICAL RESULTS FOR EACH VARIABLE, PARTITIONED BY TREATMENT PLUS ONE-SAMPLE T- TESTS ASSESSING THE IN-TREATMENT CHANGES}

Table A-1. Sample statistics for SNA Initial, showing Edgewise sample alone $(\mathrm{n}=$ 31), MARA+Edgewise alone $(n=33)$ and the total sample $(n=64)$.

\begin{tabular}{lccc}
\hline \multicolumn{1}{c}{ Statistic } & $\begin{array}{c}\text { Edgewise } \\
\text { Alone }\end{array}$ & $\begin{array}{c}\text { MARA+Edge } \\
\text { Alone }\end{array}$ & $\begin{array}{c}\text { Total } \\
\text { Sample }\end{array}$ \\
\hline Mean & 82.73 & 82.71 & 82.72 \\
Standard deviation & 3.29 & 3.23 & 3.23 \\
Standard error of mean & 0.59 & 0.56 & 0.40 \\
Upper 95\% Mean & 83.94 & 83.85 & 83.53 \\
Lower 95\% Mean & 81.52 & 81.56 & 81.91 \\
Sample size & 31 & 33 & 64 \\
Sample variance & 10.83 & 10.45 & 10.46 \\
Skewness (g1) & -0.21 & -0.81 & -0.50 \\
Kurtosis (g2) & -0.36 & 0.53 & -0.01 \\
Coef Variation & 3.98 & 3.91 & 3.91 \\
Number missing & 0 & 0 & 0 \\
Median & 82.6 & 82.9 & 82.9 \\
Mode & 80.8 & 81.7 & 81.9 \\
\hline
\end{tabular}


Table A-2. Sample statistics for SNA Final, showing Edgewise sample alone (n= 31), MARA+Edgewise alone $(n=33)$ and the total sample $(n=64)$.

\begin{tabular}{lccc}
\hline \multicolumn{1}{c}{ Statistic } & $\begin{array}{c}\text { Edgewise } \\
\text { Alone }\end{array}$ & $\begin{array}{c}\text { MARA+Edge } \\
\text { Alone }\end{array}$ & $\begin{array}{c}\text { Total } \\
\text { Sample }\end{array}$ \\
\hline Mean & 80.48 & 81.90 & 81.21 \\
Standard deviation & 2.94 & 3.29 & 3.18 \\
Standard error of mean & 0.53 & 0.57 & 0.40 \\
Upper 95\% Mean & 81.56 & 83.07 & 82.01 \\
Lower 95\% Mean & 79.40 & 80.73 & 80.42 \\
Sample size & 31 & 33 & 64 \\
Sample variance & 8.65 & 10.80 & 10.12 \\
Skewness (g1) & 0.02 & -0.21 & -0.03 \\
Kurtosis (g2) & -0.42 & 0.59 & 0.02 \\
Coef Variation & 3.65 & 4.01 & 3.92 \\
Number missing & 0 & 0 & 0 \\
Median & 80.3 & 81.9 & 81.3 \\
Mode & 80.2 & 80.8 & 83.3 \\
\hline
\end{tabular}


Table A-3. Sample statistics for Change in SNA, showing Edgewise sample alone $(n=31)$, MARA+Edgewise alone $(n=33)$ and the total sample $(n=64)$.

\begin{tabular}{lccc}
\hline \multicolumn{1}{c}{ Statistic } & $\begin{array}{c}\text { Edgewise } \\
\text { Alone }\end{array}$ & $\begin{array}{c}\text { MARA+Edge } \\
\text { Alone }\end{array}$ & $\begin{array}{c}\text { Total } \\
\text { Sample }\end{array}$ \\
\hline Mean & -2.25 & -0.81 & -1.51 \\
Standard deviation & 1.19 & 1.31 & 1.44 \\
Standard error of mean & 0.21 & 0.23 & 0.18 \\
Upper 95\% Mean & -1.82 & -0.34 & -1.15 \\
Lower 95\% Mean & -2.69 & -1.27 & -1.87 \\
Sample size & 31 & 33 & 64 \\
Sample variance & 1.41 & 1.72 & 2.08 \\
Skewness (g1) & 0.14 & 0.29 & 0.25 \\
Kurtosis (g2) & -0.77 & -0.11 & -0.19 \\
Coef Variation & -52.81 & -162.79 & -95.70 \\
Number missing & 0 & 0 & 0 \\
Median & -2.2 & -0.9 & -1.4 \\
Mode & -3.5 & -0.1 & -0.1 \\
& & & -8.36 \\
& & P-value (two tail) & $<0.0001$ \\
\hline
\end{tabular}


Table A-4. Sample statistics for SNB Initial, showing Edgewise sample alone ( $\mathrm{n}=$ 31), MARA+Edgewise alone $(n=33)$ and the total sample $(n=64)$.

\begin{tabular}{lccc}
\hline \multicolumn{1}{c}{ Statistic } & $\begin{array}{c}\text { Edgewise } \\
\text { Alone }\end{array}$ & $\begin{array}{c}\text { MARA+Edge } \\
\text { Alone }\end{array}$ & $\begin{array}{c}\text { Total } \\
\text { Sample }\end{array}$ \\
\hline Mean & 76.97 & 76.88 & 76.92 \\
Standard deviation & 2.77 & 3.07 & 2.91 \\
Standard error of mean & 0.50 & 0.53 & 0.36 \\
Upper 95\% Mean & 77.98 & 77.97 & 77.65 \\
Lower 95\% Mean & 75.95 & 75.79 & 76.20 \\
Sample size & 31 & 33 & 64 \\
Sample variance & 7.68 & 9.44 & 8.45 \\
Skewness (g1) & 0.27 & -0.12 & 0.03 \\
Kurtosis (g2) & 0.21 & -0.76 & -0.42 \\
Coef Variation & 3.60 & 4.00 & 3.78 \\
Number missing & 0 & 0 & 0 \\
Median & 76.6 & 77.3 & 77.0 \\
Mode & 77.3 & 78.8 & 78.8 \\
\hline
\end{tabular}


Table A-5. Sample statistics for SNB Final, showing Edgewise sample alone (n = 31), MARA+Edgewise alone $(n=33)$ and the total sample $(n=64)$.

\begin{tabular}{lccc}
\hline \multicolumn{1}{c}{ Statistic } & $\begin{array}{c}\text { Edgewise } \\
\text { Alone }\end{array}$ & $\begin{array}{c}\text { MARA+Edge } \\
\text { Alone }\end{array}$ & $\begin{array}{c}\text { Total } \\
\text { Sample }\end{array}$ \\
\hline Mean & 76.51 & 77.40 & 76.97 \\
Standard deviation & 2.78 & 3.06 & 2.94 \\
Standard error of mean & 0.50 & 0.53 & 0.37 \\
Upper 95\% Mean & 77.53 & 78.49 & 77.70 \\
Lower 95\% Mean & 75.49 & 76.32 & 76.24 \\
Sample size & 31 & 33 & 64 \\
Sample variance & 7.74 & 9.33 & 8.63 \\
Skewness (g1) & 0.28 & -0.07 & 0.11 \\
Kurtosis (g2) & 0.03 & -0.83 & -0.60 \\
Coef Variation & 3.64 & 3.95 & 3.82 \\
Number missing & 0 & 0 & 0 \\
Median & 76.2 & 77.6 & 77.1 \\
Mode & 76.1 & 73.7 & 76.1 \\
\hline
\end{tabular}


Table A-6. Sample statistics for Change in SNB, showing Edgewise sample alone $(n=31)$, MARA+Edgewise alone $(n=33)$ and the total sample $(n=64)$.

\begin{tabular}{|c|c|c|c|}
\hline Statistic & $\begin{array}{c}\text { Edgewise } \\
\text { Alone }\end{array}$ & $\begin{array}{c}\text { MARA+Edge } \\
\text { Alone } \\
\end{array}$ & $\begin{array}{c}\text { Total } \\
\text { Sample }\end{array}$ \\
\hline Mean & -0.46 & 0.52 & 0.05 \\
\hline Standard deviation & 1.10 & 1.24 & 1.26 \\
\hline Standard error of mean & 0.20 & 0.22 & 0.16 \\
\hline Upper $95 \%$ Mean & -0.06 & 0.96 & 0.36 \\
\hline Lower 95\% Mean & -0.86 & 0.09 & -0.27 \\
\hline Sample size & 31 & 33 & 64 \\
\hline Sample variance & 1.20 & 1.53 & 1.59 \\
\hline Skewness (g1) & 0.03 & -0.39 & -0.05 \\
\hline Kurtosis (g2) & -1.09 & -0.42 & -0.81 \\
\hline Coef Variation & -239.33 & 235.66 & $2,605.20$ \\
\hline Number missing & 0 & 0 & 0 \\
\hline Median & -0.4 & 0.6 & 0.2 \\
\hline \multirow[t]{3}{*}{ Mode } & -1.4 & 1.9 & -1.4 \\
\hline & & t-test & 0.31 \\
\hline & & P-value (two tail) & 0.7598 \\
\hline
\end{tabular}


Table A-7. Sample statistics for ANB Initial, showing Edgewise sample alone ( $\mathrm{n}=$ 31), MARA+Edgewise alone $(n=33)$ and the total sample $(n=64)$.

\begin{tabular}{lccc}
\hline \multicolumn{1}{c}{ Statistic } & $\begin{array}{c}\text { Edgewise } \\
\text { Alone }\end{array}$ & $\begin{array}{c}\text { MARA+Edge } \\
\text { Alone }\end{array}$ & $\begin{array}{c}\text { Total } \\
\text { Sample }\end{array}$ \\
\hline Mean & 5.76 & 5.84 & 5.80 \\
Standard deviation & 1.62 & 1.43 & 1.52 \\
Standard error of mean & 0.29 & 0.25 & 0.19 \\
Upper 95\% Mean & 6.36 & 6.34 & 6.18 \\
Lower 95\% Mean & 5.17 & 5.33 & 5.42 \\
Sample size & 31 & 33 & 64 \\
Sample variance & 2.64 & 2.05 & 2.30 \\
Skewness (g1) & 1.55 & 0.31 & 0.99 \\
Kurtosis (g2) & 2.08 & -0.66 & 0.79 \\
Coef Variation & 28.18 & 24.55 & 26.14 \\
Number missing & 0 & 0 & 0 \\
Median & 5.3 & 5.5 & 5.5 \\
Mode & 4.5 & 5.5 & 5.3 \\
\hline
\end{tabular}


Table A-8. Sample statistics for ANB Final, showing Edgewise sample alone (n= 31), MARA+Edgewise alone $(n=33)$ and the total sample $(n=64)$.

\begin{tabular}{lccc}
\hline \multicolumn{1}{c}{ Statistic } & $\begin{array}{c}\text { Edgewise } \\
\text { Alone }\end{array}$ & $\begin{array}{c}\text { MARA+Edge } \\
\text { Alone }\end{array}$ & $\begin{array}{c}\text { Total } \\
\text { Sample }\end{array}$ \\
\hline Mean & 3.97 & 4.49 & 4.24 \\
Standard deviation & 1.64 & 1.63 & 1.64 \\
Standard error of mean & 0.29 & 0.28 & 0.21 \\
Upper 95\% Mean & 4.57 & 5.07 & 4.65 \\
Lower 95\% Mean & 3.37 & 3.91 & 3.83 \\
Sample size & 31 & 33 & 64 \\
Sample variance & 2.68 & 2.65 & 2.69 \\
Skewness (g1) & 1.38 & 0.22 & 0.73 \\
Kurtosis (g2) & 2.41 & -1.02 & 0.11 \\
Coef Variation & 41.24 & 36.25 & 38.71 \\
Number missing & 0 & 0 & 0 \\
Median & 4.0 & 4.3 & 4.2 \\
Mode & 4.5 & 4.2 & 4.2 \\
\hline
\end{tabular}


Table A-9. Sample statistics for Change in ANB, showing Edgewise sample alone $(n=31)$, MARA+Edgewise alone $(n=33)$ and the total sample $(n=64)$.

\begin{tabular}{lccc}
\hline \multicolumn{1}{c}{ Statistic } & $\begin{array}{c}\text { Edgewise } \\
\text { Alone }\end{array}$ & $\begin{array}{c}\text { MARA+Edge } \\
\text { Alone }\end{array}$ & $\begin{array}{c}\text { Total } \\
\text { Sample }\end{array}$ \\
\hline Mean & -1.79 & -1.35 & -1.56 \\
Standard deviation & 0.92 & 1.28 & 1.14 \\
Standard error of mean & 0.17 & 0.22 & 0.14 \\
Upper 95\% Mean & -1.45 & -0.89 & -1.28 \\
Lower 95\% Mean & -2.13 & -1.80 & -1.85 \\
Sample size & 31 & 33 & 64 \\
Sample variance & 0.85 & 1.65 & 1.29 \\
Skewness (g1) & -0.60 & -0.11 & -0.04 \\
Kurtosis (g2) & 0.41 & -0.46 & -0.01 \\
Coef Variation & -51.46 & -95.47 & -72.82 \\
Number missing & 0 & 0 & 0 \\
Median & -1.9 & -1.3 & -1.5 \\
Mode & -2.1 & -1.5 & -1.5 \\
& & & t-test \\
& & -10.99 \\
& & & $<0.0001$ \\
\hline
\end{tabular}


Table A-10. Sample statistics for Overbite Initial, showing Edgewise sample alone $(n=31)$, MARA+Edgewise alone $(n=33)$ and the total sample $(n=64)$.

\begin{tabular}{lccc}
\hline \multicolumn{1}{c}{ Statistic } & $\begin{array}{c}\text { Edgewise } \\
\text { Alone }\end{array}$ & $\begin{array}{c}\text { MARA+Edge } \\
\text { Alone }\end{array}$ & $\begin{array}{c}\text { Total } \\
\text { Sample }\end{array}$ \\
\hline Mean & 3.72 & 3.49 & 3.60 \\
Standard deviation & 1.44 & 2.24 & 1.88 \\
Standard error of mean & 0.26 & 0.39 & 0.24 \\
Upper 95\% Mean & 4.25 & 4.28 & 4.07 \\
Lower 95\% Mean & 3.19 & 2.70 & 3.13 \\
Sample size & 31 & 33 & 64 \\
Sample variance & 2.09 & 5.00 & 3.55 \\
Skewness (g1) & -0.05 & -0.75 & -0.71 \\
Kurtosis (g2) & 0.12 & -0.31 & 0.30 \\
Coef Variation & 38.82 & 64.05 & 52.27 \\
Number missing & 0 & 0 & 0 \\
Median & 3.6 & 4.0 & 3.7 \\
Mode & 2.6 & 4.8 & 2.6 \\
\hline
\end{tabular}


Table A-11. Sample statistics for Overbite Final, showing Edgewise sample alone $(n=31)$, MARA+Edgewise alone $(n=33)$ and the total sample $(n=64)$.

\begin{tabular}{lccc}
\hline \multicolumn{1}{c}{ Statistic } & $\begin{array}{c}\text { Edgewise } \\
\text { Alone }\end{array}$ & $\begin{array}{c}\text { MARA+Edge } \\
\text { Alone }\end{array}$ & $\begin{array}{c}\text { Total } \\
\text { Sample }\end{array}$ \\
\hline Mean & 1.38 & 1.46 & 1.42 \\
Standard deviation & 0.76 & 1.24 & 1.03 \\
Standard error of mean & 0.14 & 0.22 & 0.13 \\
Upper 95\% Mean & 1.66 & 1.90 & 1.68 \\
Lower 95\% Mean & 1.10 & 1.02 & 1.16 \\
Sample size & 31 & 33 & 64 \\
Sample variance & 0.58 & 1.54 & 1.06 \\
Skewness (g1) & 0.08 & -0.18 & -0.09 \\
Kurtosis (g2) & -0.13 & -0.01 & 0.47 \\
Coef Variation & 55.05 & 85.05 & 72.39 \\
Number missing & 0 & 0 & 0 \\
Median & 1.1 & 1.3 & 1.3 \\
Mode & 1.0 & 0.3 & 1.0 \\
\hline
\end{tabular}


Table A-12. Sample statistics for Change in Overbite, showing Edgewise sample alone $(n=31)$, MARA+Edgewise alone $(n=33)$ and the total sample $(n=64)$.

\begin{tabular}{lccc}
\hline \multicolumn{1}{c}{ Statistic } & $\begin{array}{c}\text { Edgewise } \\
\text { Alone }\end{array}$ & $\begin{array}{c}\text { MARA+Edge } \\
\text { Alone }\end{array}$ & $\begin{array}{c}\text { Total } \\
\text { Sample }\end{array}$ \\
\hline Mean & -2.34 & -2.03 & -2.18 \\
Standard deviation & 1.54 & 1.97 & 1.77 \\
Standard error of mean & 0.28 & 0.34 & 0.22 \\
Upper 95\% Mean & -1.78 & -1.34 & -1.74 \\
Lower 95\% Mean & -2.91 & -2.73 & -2.62 \\
Sample size & 31 & 33 & 64 \\
Sample variance & 2.38 & 3.87 & 3.12 \\
Skewness (g1) & -0.48 & 0.30 & 0.12 \\
Kurtosis (g2) & 0.58 & 0.11 & 0.43 \\
Coef Variation & -65.93 & -96.70 & -80.96 \\
Number missing & 0 & 0 & 0 \\
Median & -2.3 & -2.3 & -2.3 \\
Mode & -2.4 & -0.4 & -2.0 \\
& & P-value (two tail) & -9.88 \\
& & & $<0.0001$ \\
\hline
\end{tabular}


Table A-13. Sample statistics for Overjet Initial, showing Edgewise sample alone $(n=31)$, MARA+Edgewise alone $(n=33)$ and the total sample $(n=64)$.

\begin{tabular}{lccc}
\hline \multicolumn{1}{c}{ Statistic } & $\begin{array}{c}\text { Edgewise } \\
\text { Alone }\end{array}$ & $\begin{array}{c}\text { MARA+Edge } \\
\text { Alone }\end{array}$ & $\begin{array}{c}\text { Total } \\
\text { Sample }\end{array}$ \\
\hline Mean & 6.17 & 5.85 & 6.00 \\
Standard deviation & 1.86 & 1.95 & 1.90 \\
Standard error of mean & 0.33 & 0.34 & 0.24 \\
Upper 95\% Mean & 6.85 & 6.54 & 6.48 \\
Lower 95\% Mean & 5.48 & 5.15 & 5.53 \\
Sample size & 31 & 33 & 64 \\
Sample variance & 3.47 & 3.81 & 3.61 \\
Skewness (g1) & 0.59 & 0.98 & 0.76 \\
Kurtosis (g2) & -0.75 & 1.37 & 0.23 \\
Coef Variation & 30.21 & 33.37 & 31.67 \\
Number missing & 0 & 0 & 0 \\
Median & 5.8 & 5.7 & 5.8 \\
Mode & 4.2 & 3.8 & 4.2 \\
\hline
\end{tabular}


Table A-14. Sample statistics for Overjet Final, showing Edgewise sample alone (n $=31)$, MARA+Edgewise alone $(n=33)$ and the total sample $(n=64)$.

\begin{tabular}{lccc}
\hline \multicolumn{1}{c}{ Statistic } & $\begin{array}{c}\text { Edgewise } \\
\text { Alone }\end{array}$ & $\begin{array}{c}\text { MARA+Edge } \\
\text { Alone }\end{array}$ & $\begin{array}{c}\text { Total } \\
\text { Sample }\end{array}$ \\
\hline Mean & 2.63 & 3.17 & 2.91 \\
Standard deviation & 0.81 & 0.99 & 0.94 \\
Standard error of mean & 0.15 & 0.17 & 0.12 \\
Upper 95\% Mean & 2.93 & 3.52 & 3.14 \\
Lower 95\% Mean & 2.33 & 2.82 & 2.67 \\
Sample size & 31 & 33 & 64 \\
Sample variance & 0.66 & 0.98 & 0.89 \\
Skewness (g1) & -0.08 & 0.68 & 0.52 \\
Kurtosis (g2) & -0.34 & 0.39 & 0.64 \\
Coef Variation & 30.98 & 31.18 & 32.37 \\
Number missing & 0 & 0 & 0 \\
Median & 2.6 & 3.0 & 2.9 \\
Mode & 2.9 & 2.4 & 2.9 \\
\hline
\end{tabular}


Table A-15. Sample statistics for Change in Overjet showing Edgewise sample alone $(n=31)$, MARA+Edgewise alone $(n=33)$ and the total sample $(n=64)$.

\begin{tabular}{|c|c|c|c|}
\hline Statistic & $\begin{array}{c}\text { Edgewise } \\
\text { Alone }\end{array}$ & $\begin{array}{c}\text { MARA+Edge } \\
\text { Alone }\end{array}$ & $\begin{array}{c}\text { Total } \\
\text { Sample }\end{array}$ \\
\hline Mean & -3.54 & -2.68 & -3.09 \\
\hline Standard deviation & 1.75 & 2.08 & 1.96 \\
\hline Standard error of mean & 0.31 & 0.36 & 0.24 \\
\hline Upper 95\% Mean & -2.90 & -1.94 & -2.60 \\
\hline Lower 95\% Mean & -4.18 & -3.41 & -3.58 \\
\hline Sample size & 31 & 33 & 64 \\
\hline Sample variance & 3.06 & 4.31 & 3.84 \\
\hline Skewness (g1) & -0.41 & -0.69 & -0.43 \\
\hline Kurtosis (g2) & -0.95 & 0.99 & 0.08 \\
\hline Coef Variation & -49.40 & -77.63 & -63.30 \\
\hline Number missing & 0 & 0 & 0 \\
\hline Median & -3.1 & -2.5 & -2.9 \\
\hline \multirow[t]{3}{*}{ Mode } & -2.8 & -3.2 & -4.9 \\
\hline & & t-test & -12.64 \\
\hline & & P-value (two tail) & $<0.0001$ \\
\hline
\end{tabular}


Table A-16. Sample statistics for Mesial Molar Relation Initial, showing Edgewise sample alone $(n=31)$, MARA+Edgewise alone $(n=33)$ and the total sample $(n=$ 64).

\begin{tabular}{lccc}
\hline \multicolumn{1}{c}{ Statistic } & $\begin{array}{c}\text { Edgewise } \\
\text { Alone }\end{array}$ & $\begin{array}{c}\text { MARA+Edge } \\
\text { Alone }\end{array}$ & $\begin{array}{c}\text { Total } \\
\text { Sample }\end{array}$ \\
\hline Mean & 1.32 & 1.47 & 1.40 \\
Standard deviation & 1.30 & 1.22 & 1.26 \\
Standard error of mean & 0.23 & 0.21 & 0.16 \\
Upper 95\% Mean & 1.80 & 1.90 & 1.71 \\
Lower 95\% Mean & 0.84 & 1.04 & 1.08 \\
Sample size & 31 & 33 & 64 \\
Sample variance & 1.70 & 1.50 & 1.58 \\
Skewness (g1) & 0.06 & -0.73 & -0.32 \\
Kurtosis (g2) & -0.85 & 1.92 & 0.20 \\
Coef Variation & 98.87 & 83.22 & 89.87 \\
Number missing & 0 & 0 & 0 \\
Median & 1.4 & 1.3 & 1.4 \\
Mode & 0.1 & 0.6 & 0.6 \\
\hline
\end{tabular}


Table A-17. Sample statistics for Mesial Molar Relation Final, showing Edgewise sample alone $(n=31)$, MARA + Edgewise alone $(n=33)$ and the total sample $(n=$ 64).

\begin{tabular}{lccc}
\hline \multicolumn{1}{c}{ Statistic } & $\begin{array}{c}\text { Edgewise } \\
\text { Alone }\end{array}$ & $\begin{array}{c}\text { MARA+Edge } \\
\text { Alone }\end{array}$ & $\begin{array}{c}\text { Total } \\
\text { Sample }\end{array}$ \\
\hline Mean & -1.15 & -0.31 & -0.72 \\
Standard deviation & 1.11 & 0.97 & 1.12 \\
Standard error of mean & 0.20 & 0.17 & 0.14 \\
Upper 95\% Mean & -0.75 & 0.04 & -0.44 \\
Lower 95\% Mean & -1.56 & -0.65 & -1.00 \\
Sample size & 31 & 33 & 64 \\
Sample variance & 1.23 & 0.95 & 1.25 \\
Skewness (g1) & 0.38 & -0.37 & -0.08 \\
Kurtosis (g2) & -0.03 & 1.09 & -0.24 \\
Coef Variation & -96.03 & -318.56 & -155.97 \\
Number missing & 0 & 0 & 0 \\
Median & -1.2 & -0.3 & -0.7 \\
Mode & -1.8 & 0.3 & -0.6 \\
\hline
\end{tabular}


Table A-18. Sample statistics for Change in Molar Relationship, showing Edgewise sample alone $(n=31)$, MARA+Edgewise alone $(n=33)$ and the total sample $(n=64)$.

\begin{tabular}{|c|c|c|c|}
\hline Statistic & $\begin{array}{c}\text { Edgewise } \\
\text { Alone }\end{array}$ & $\begin{array}{c}\text { MARA+Edge } \\
\text { Alone }\end{array}$ & $\begin{array}{c}\text { Total } \\
\text { Sample }\end{array}$ \\
\hline Mean & -2.47 & -1.78 & -2.11 \\
\hline Standard deviation & 1.53 & 1.39 & 1.49 \\
\hline Standard error of mean & 0.27 & 0.24 & 0.19 \\
\hline Upper 95\% Mean & -1.91 & -1.28 & -1.74 \\
\hline Lower 95\% Mean & -3.04 & -2.27 & -2.49 \\
\hline Sample size & 31 & 33 & 64 \\
\hline Sample variance & 2.34 & 1.92 & 2.21 \\
\hline Skewness (g1) & -0.59 & -0.29 & -0.48 \\
\hline Kurtosis (g2) & 0.47 & 0.94 & 0.69 \\
\hline Coef Variation & -61.83 & -78.03 & -70.37 \\
\hline Number missing & 0 & 0 & 0 \\
\hline Median & -2.2 & -1.6 & -2.0 \\
\hline \multirow[t]{3}{*}{ Mode } & -2.2 & -3.1 & -2.0 \\
\hline & & t-test & -11.37 \\
\hline & & P-value (two tail) & $<0.0001$ \\
\hline
\end{tabular}


Table A-19. Sample statistics for Sella-Vertical-M Initial, showing Edgewise sample alone $(n=31)$, MARA+Edgewise alone $(n=33)$ and the total sample $(n=$ 64).

\begin{tabular}{lccc}
\hline \multicolumn{1}{c}{ Statistic } & $\begin{array}{c}\text { Edgewise } \\
\text { Alone }\end{array}$ & $\begin{array}{c}\text { MARA+Edge } \\
\text { Alone }\end{array}$ & $\begin{array}{c}\text { Total } \\
\text { Sample }\end{array}$ \\
\hline Mean & 45.82 & 44.93 & 45.36 \\
Standard deviation & 3.84 & 4.89 & 4.40 \\
Standard error of mean & 0.69 & 0.85 & 0.55 \\
Upper 95\% Mean & 47.23 & 46.66 & 46.46 \\
Lower 95\% Mean & 44.41 & 43.19 & 44.26 \\
Sample size & 31 & 33 & 64 \\
Sample variance & 14.74 & 23.90 & 19.36 \\
Skewness (g1) & 0.00 & -0.52 & -0.42 \\
Kurtosis (g2) & -1.10 & 0.91 & 0.59 \\
Coef Variation & 8.38 & 10.88 & 9.70 \\
Number missing & 0 & 0 & 0 \\
Median & 45.9 & 45.4 & 45.5 \\
Mode & 47.2 & 36.6 & 45.4 \\
\hline
\end{tabular}


Table A-20. Sample statistics for Sella-Vertical-M Final, showing Edgewise sample alone $(n=31)$, MARA+Edgewise alone $(n=33)$ and the total sample $(n=64)$.

\begin{tabular}{lccc}
\hline \multicolumn{1}{c}{ Statistic } & $\begin{array}{c}\text { Edgewise } \\
\text { Alone }\end{array}$ & $\begin{array}{c}\text { MARA+Edge } \\
\text { Alone }\end{array}$ & $\begin{array}{c}\text { Total } \\
\text { Sample }\end{array}$ \\
\hline Mean & 51.90 & 47.51 & 49.64 \\
Standard deviation & 5.06 & 5.65 & 5.77 \\
Standard error of mean & 0.91 & 0.98 & 0.72 \\
Upper 95\% Mean & 53.76 & 49.51 & 51.08 \\
Lower 95\% Mean & 50.05 & 45.51 & 48.20 \\
Sample size & 31 & 33 & 64 \\
Sample variance & 25.59 & 31.93 & 33.30 \\
Skewness (g1) & 0.07 & -0.34 & -0.25 \\
Kurtosis (g2) & -1.31 & 0.35 & 0.04 \\
Coef Variation & 9.75 & 11.89 & 11.63 \\
Number missing & 0 & 0 & 0 \\
Median & 51.4 & 47.8 & 49.2 \\
Mode & 56.4 & 41.2 & 49.6 \\
\hline
\end{tabular}


Table A-21. Sample statistics for Change in Sella-Vertical-M, showing Edgewise sample alone $(n=31)$, MARA+Edgewise alone $(n=33)$ and the total sample $(n=$ 64).

\begin{tabular}{lccc}
\hline \multicolumn{1}{c}{ Statistic } & $\begin{array}{c}\text { Edgewise } \\
\text { Alone }\end{array}$ & $\begin{array}{c}\text { MARA+Edge } \\
\text { Alone }\end{array}$ & $\begin{array}{c}\text { Total } \\
\text { Sample }\end{array}$ \\
\hline Mean & 6.08 & 2.58 & 4.28 \\
Standard deviation & 3.41 & 2.69 & 3.51 \\
Standard error of mean & 0.61 & 0.47 & 0.44 \\
Upper 95\% Mean & 7.33 & 3.54 & 5.15 \\
Lower 95\% Mean & 4.83 & 1.63 & 3.40 \\
Sample size & 31 & 33 & 64 \\
Sample variance & 11.64 & 7.24 & 12.33 \\
Skewness (g1) & -0.59 & -0.44 & -0.07 \\
Kurtosis (g2) & 1.17 & 0.34 & 0.12 \\
Coef Variation & 56.11 & 104.22 & 82.10 \\
Number missing & 0 & 0 & 0 \\
Median & 6.3 & 2.7 & 4.6 \\
Mode & 6.2 & 0.5 & 4.6 \\
& & & t-test \\
& & & 9.74 \\
& & & $<0.0001$ \\
\hline
\end{tabular}


Table A-22. Sample statistics for Axial ML Left Initial, showing Edgewise sample alone $(n=31)$, MARA+Edgewise alone $(n=33)$ and the total sample $(n=64)$.

\begin{tabular}{lccc}
\hline \multicolumn{1}{c}{ Statistic } & $\begin{array}{c}\text { Edgewise } \\
\text { Alone }\end{array}$ & $\begin{array}{c}\text { MARA+Edge } \\
\text { Alone }\end{array}$ & $\begin{array}{c}\text { Total } \\
\text { Sample }\end{array}$ \\
\hline Mean & 17.43 & 17.06 & 17.24 \\
Standard deviation & 1.80 & 2.29 & 2.06 \\
Standard error of mean & 0.32 & 0.40 & 0.26 \\
Upper 95\% Mean & 18.09 & 17.88 & 17.76 \\
Lower 95\% Mean & 16.77 & 16.25 & 16.73 \\
Sample size & 31 & 33 & 64 \\
Sample variance & 3.25 & 5.25 & 4.25 \\
Skewness (g1) & 0.05 & -0.18 & -0.17 \\
Kurtosis (g2) & -0.55 & -0.20 & -0.16 \\
Coef Variation & 10.34 & 13.43 & 11.96 \\
Number missing & 0 & 0 & 0 \\
Median & 17.2 & 16.8 & 17.0 \\
Mode & 16.4 & 16.5 & 16.4 \\
\hline
\end{tabular}


Table A-23. Sample statistics for Axial ML Left Final, showing Edgewise sample alone $(n=31)$, MARA+Edgewise alone $(n=33)$ and the total sample $(n=64)$.

\begin{tabular}{lccc}
\hline \multicolumn{1}{c}{ Statistic } & $\begin{array}{c}\text { Edgewise } \\
\text { Alone }\end{array}$ & $\begin{array}{c}\text { MARA+Edge } \\
\text { Alone }\end{array}$ & $\begin{array}{c}\text { Total } \\
\text { Sample }\end{array}$ \\
\hline Mean & 18.39 & 17.73 & 18.05 \\
Standard deviation & 2.03 & 2.63 & 2.36 \\
Standard error of mean & 0.36 & 0.46 & 0.30 \\
Upper 95\% Mean & 19.13 & 18.66 & 18.64 \\
Lower 95\% Mean & 17.64 & 16.80 & 17.46 \\
Sample size & 31 & 33 & 64 \\
Sample variance & 4.10 & 6.94 & 5.59 \\
Skewness (g1) & -0.07 & -0.17 & -0.24 \\
Kurtosis (g2) & -0.64 & 0.14 & 0.11 \\
Coef Variation & 11.02 & 14.85 & 13.10 \\
Number missing & 0 & 0 & 0 \\
Median & 18.0 & 17.9 & 18.0 \\
Mode & 17.5 & 16.0 & 17.5 \\
\hline
\end{tabular}


Table A-24. Sample statistics for Change in Axial ML Left, showing Edgewise sample alone $(n=31)$, MARA + Edgewise alone $(n=33)$ and the total sample $(n=$ 64).

\begin{tabular}{|c|c|c|c|}
\hline Statistic & $\begin{array}{c}\text { Edgewise } \\
\text { Alone }\end{array}$ & $\begin{array}{c}\text { MARA+Edge } \\
\text { Alone } \\
\end{array}$ & $\begin{array}{c}\text { Total } \\
\text { Sample }\end{array}$ \\
\hline Mean & 0.95 & 0.67 & 0.81 \\
\hline Standard deviation & 0.87 & 0.89 & 0.89 \\
\hline Standard error of mean & 0.16 & 0.15 & 0.11 \\
\hline Upper 95\% Mean & 1.28 & 0.98 & 1.03 \\
\hline Lower 95\% Mean & 0.63 & 0.35 & 0.58 \\
\hline Sample size & 31 & 33 & 64 \\
\hline Sample variance & 0.76 & 0.79 & 0.79 \\
\hline Skewness (g1) & 0.77 & 0.55 & 0.61 \\
\hline Kurtosis (g2) & 0.15 & -0.64 & -0.27 \\
\hline Coef Variation & 91.57 & 133.36 & 110.01 \\
\hline Number missing & 0 & 0 & 0 \\
\hline Median & 0.9 & 0.4 & 0.7 \\
\hline \multirow[t]{3}{*}{ Mode } & 0.2 & -0.2 & 0.2 \\
\hline & & t-test & 7.27 \\
\hline & & P-value (two tail) & $<0.0001$ \\
\hline
\end{tabular}


Table A-25. Sample statistics for Axial ML Right Initial, showing Edgewise sample alone $(n=31)$, MARA + Edgewise alone $(n=33)$ and the total sample $(n=$ 64).

\begin{tabular}{lccc}
\hline \multicolumn{1}{c}{ Statistic } & $\begin{array}{c}\text { Edgewise } \\
\text { Alone }\end{array}$ & $\begin{array}{c}\text { MARA+Edge } \\
\text { Alone }\end{array}$ & $\begin{array}{c}\text { Total } \\
\text { Sample }\end{array}$ \\
\hline Mean & 17.65 & 17.09 & 17.36 \\
Standard deviation & 1.78 & 2.32 & 2.08 \\
Standard error of mean & 0.32 & 0.40 & 0.26 \\
Upper 95\% Mean & 18.30 & 17.92 & 17.88 \\
Lower 95\% Mean & 17.00 & 16.27 & 16.84 \\
Sample size & 31 & 33 & 64 \\
Sample variance & 3.17 & 5.38 & 4.32 \\
Skewness (g1) & 0.75 & 0.08 & 0.16 \\
Kurtosis (g2) & 0.09 & 0.08 & 0.28 \\
Coef Variation & 10.08 & 13.57 & 11.97 \\
Number missing & 0 & 0 & 0 \\
Median & 17.3 & 16.9 & 17.2 \\
Mode & 17.3 & 15.1 & 18.8 \\
\hline
\end{tabular}


Table A-26. Sample statistics for Axial ML Right Final, showing Edgewise sample alone $(n=31)$, MARA+Edgewise alone $(n=33)$ and the total sample $(n=64)$.

\begin{tabular}{lccc}
\hline \multicolumn{1}{c}{ Statistic } & $\begin{array}{c}\text { Edgewise } \\
\text { Alone }\end{array}$ & $\begin{array}{c}\text { MARA+Edge } \\
\text { Alone }\end{array}$ & $\begin{array}{c}\text { Total } \\
\text { Sample }\end{array}$ \\
\hline Mean & 18.44 & 17.95 & 18.19 \\
Standard deviation & 1.64 & 2.48 & 2.12 \\
Standard error of mean & 0.29 & 0.43 & 0.26 \\
Upper 95\% Mean & 19.04 & 18.83 & 18.72 \\
Lower 95\% Mean & 17.84 & 17.07 & 17.66 \\
Sample size & 31 & 33 & 64 \\
Sample variance & 2.70 & 6.16 & 4.47 \\
Skewness (g1) & 0.17 & 0.14 & 0.01 \\
Kurtosis (g2) & -0.29 & 0.10 & 0.36 \\
Coef Variation & 8.91 & 13.83 & 11.63 \\
Number missing & 0 & 0 & 0 \\
Median & 18.3 & 17.9 & 18.2 \\
Mode & 17.6 & 16.4 & 16.5 \\
\hline
\end{tabular}


Table A-27. Sample statistics for Change in Axial ML R, showing Edgewise sample alone $(n=31)$, MARA + Edgewise alone $(n=33)$ and the total sample $(n=$ 64).

\begin{tabular}{|c|c|c|c|}
\hline Statistic & $\begin{array}{c}\text { Edgewise } \\
\text { Alone }\end{array}$ & $\begin{array}{c}\text { MARA+Edge } \\
\text { Alone }\end{array}$ & $\begin{array}{c}\text { Total } \\
\text { Sample } \\
\end{array}$ \\
\hline Mean & 0.79 & 0.85 & 0.82 \\
\hline Standard deviation & 1.16 & 0.79 & 0.98 \\
\hline Standard error of mean & 0.21 & 0.14 & 0.12 \\
\hline Upper 95\% Mean & 1.22 & 1.13 & 1.07 \\
\hline Lower 95\% Mean & 0.36 & 0.58 & 0.58 \\
\hline Sample size & 31 & 33 & 64 \\
\hline Sample variance & 1.35 & 0.62 & 0.96 \\
\hline Skewness (g1) & 0.02 & 0.34 & 0.07 \\
\hline Kurtosis (g2) & 0.80 & 0.11 & 1.03 \\
\hline Coef Variation & 146.77 & 92.01 & 118.73 \\
\hline Number missing & 0 & 0 & 0 \\
\hline Median & 0.5 & 0.9 & 0.7 \\
\hline \multirow[t]{3}{*}{ Mode } & 0.4 & 0.3 & 0.3 \\
\hline & & t-test & 6.74 \\
\hline & & P-value (two tail) & $<0.0001$ \\
\hline
\end{tabular}

The $t$ test assessed whether the average in-treatment change differed systematically from zero (two tail). 
Table A-28. Sample statistics for Axial AP Left Initial, showing Edgewise sample alone $(n=31)$, MARA+Edgewise alone $(n=33)$ and the total sample $(n=64)$.

\begin{tabular}{lccc}
\hline \multicolumn{1}{c}{ Statistic } & $\begin{array}{c}\text { Edgewise } \\
\text { Alone }\end{array}$ & $\begin{array}{c}\text { MARA+Edge } \\
\text { Alone }\end{array}$ & $\begin{array}{c}\text { Total } \\
\text { Sample }\end{array}$ \\
\hline Mean & 6.67 & 7.03 & 6.86 \\
Standard deviation & 1.22 & 1.18 & 1.20 \\
Standard error of mean & 0.22 & 0.21 & 0.15 \\
Upper 95\% Mean & 7.12 & 7.45 & 7.16 \\
Lower 95\% Mean & 6.23 & 6.61 & 6.56 \\
Sample size & 31 & 33 & 64 \\
Sample variance & 1.49 & 1.39 & 1.45 \\
Skewness (g1) & -0.07 & 0.19 & 0.04 \\
Kurtosis (g2) & -0.27 & 0.20 & -0.04 \\
Coef Variation & 18.30 & 16.77 & 17.55 \\
Number missing & 0 & 0 & 0 \\
Median & 6.7 & 6.8 & 6.8 \\
Mode & 6.1 & 5.9 & 6.1 \\
\hline
\end{tabular}


Table A-29. Sample statistics for Axial AP Left Final, showing Edgewise sample alone $(n=31)$, MARA+Edgewise alone $(n=33)$ and the total sample $(n=64)$.

\begin{tabular}{lccc}
\hline \multicolumn{1}{c}{ Statistic } & $\begin{array}{c}\text { Edgewise } \\
\text { Alone }\end{array}$ & $\begin{array}{c}\text { MARA+Edge } \\
\text { Alone }\end{array}$ & $\begin{array}{c}\text { Total } \\
\text { Sample }\end{array}$ \\
\hline Mean & 6.67 & 6.75 & 6.71 \\
Standard deviation & 1.30 & 1.07 & 1.18 \\
Standard error of mean & 0.23 & 0.19 & 0.15 \\
Upper 95\% Mean & 7.15 & 7.13 & 7.01 \\
Lower 95\% Mean & 6.20 & 6.37 & 6.42 \\
Sample size & 31 & 33 & 64 \\
Sample variance & 1.69 & 1.15 & 1.39 \\
Skewness (g1) & 0.33 & 0.05 & 0.20 \\
Kurtosis (g2) & -0.08 & 0.15 & -0.02 \\
Coef Variation & 19.48 & 15.92 & 17.58 \\
Number missing & 0 & 0 & 0 \\
Median & 6.6 & 6.7 & 6.7 \\
Mode & 5.0 & 6.7 & 6.7 \\
\hline
\end{tabular}


Table A-30. Sample statistics for Change in Axial AP L, showing Edgewise sample alone $(n=31)$, MARA+Edgewise alone $(n=33)$ and the total sample $(n=64)$.

\begin{tabular}{|c|c|c|c|}
\hline Statistic & $\begin{array}{c}\text { Edgewise } \\
\text { Alone }\end{array}$ & $\begin{array}{c}\text { MARA+Edge } \\
\text { Alone }\end{array}$ & $\begin{array}{c}\text { Total } \\
\text { Sample }\end{array}$ \\
\hline Mean & 0.00 & -0.28 & -0.15 \\
\hline Standard deviation & 0.59 & 0.92 & 0.78 \\
\hline Standard error of mean & 0.11 & 0.16 & 0.10 \\
\hline Upper 95\% Mean & 0.22 & 0.04 & 0.05 \\
\hline Lower 95\% Mean & -0.22 & -0.61 & -0.34 \\
\hline Sample size & 31 & 33 & 64 \\
\hline Sample variance & 0.35 & 0.84 & 0.62 \\
\hline Skewness (g1) & 0.35 & -0.88 & -0.84 \\
\hline Kurtosis (g2) & -0.99 & 1.23 & 1.87 \\
\hline Coef Variation & . & -325.27 & -539.77 \\
\hline Number missing & 0 & 0 & 0 \\
\hline Median & -0.2 & -0.2 & -0.2 \\
\hline \multirow[t]{2}{*}{ Mode } & -0.5 & $\begin{array}{c}-0.2 \\
\text { One-sample t-test }\end{array}$ & $\begin{array}{l}-0.5 \\
1.48\end{array}$ \\
\hline & & P-value (two tail) & 0.1433 \\
\hline
\end{tabular}


Table A-31. Sample statistics for Axial AP Right Initial, showing Edgewise sample alone $(n=31)$, MARA+Edgewise alone $(n=33)$ and the total sample $(n=64)$.

\begin{tabular}{lccc}
\hline \multicolumn{1}{c}{ Statistic } & $\begin{array}{c}\text { Edgewise } \\
\text { Alone }\end{array}$ & $\begin{array}{c}\text { MARA+Edge } \\
\text { Alone }\end{array}$ & $\begin{array}{c}\text { Total } \\
\text { Sample }\end{array}$ \\
\hline Mean & 6.77 & 7.13 & 6.95 \\
Standard deviation & 1.19 & 1.06 & 1.13 \\
Standard error of mean & 0.21 & 0.18 & 0.14 \\
Upper 95\% Mean & 7.20 & 7.51 & 7.24 \\
Lower 95\% Mean & 6.33 & 6.76 & 6.67 \\
Sample size & 31 & 33 & 64 \\
Sample variance & 1.41 & 1.12 & 1.27 \\
Skewness (g1) & 0.04 & 1.03 & 0.38 \\
Kurtosis (g2) & -0.43 & 1.41 & 0.49 \\
Coef Variation & 17.53 & 14.83 & 16.21 \\
Number missing & 0 & 0 & 0 \\
Median & 6.9 & 6.9 & 6.9 \\
Mode & 6.9 & 6.5 & 6.9 \\
\hline
\end{tabular}


Table A-32. Sample statistics for Axial AP Right Final, showing Edgewise sample alone $(n=31)$, MARA+Edgewise alone $(n=33)$ and the total sample $(n=64)$.

\begin{tabular}{lccc}
\hline \multicolumn{1}{c}{ Statistic } & $\begin{array}{c}\text { Edgewise } \\
\text { Alone }\end{array}$ & $\begin{array}{c}\text { MARA+Edge } \\
\text { Alone }\end{array}$ & $\begin{array}{c}\text { Total } \\
\text { Sample }\end{array}$ \\
\hline Mean & 6.73 & 6.72 & 6.72 \\
Standard deviation & 1.11 & 1.19 & 1.14 \\
Standard error of mean & 0.20 & 0.21 & 0.14 \\
Upper 95\% Mean & 7.14 & 7.14 & 7.01 \\
Lower 95\% Mean & 6.32 & 6.29 & 6.44 \\
Sample size & 31 & 33 & 64 \\
Sample variance & 1.24 & 1.41 & 1.31 \\
Skewness (g1) & -0.17 & -0.19 & -0.18 \\
Kurtosis (g2) & -0.92 & 0.17 & -0.35 \\
Coef Variation & 16.56 & 17.69 & 17.01 \\
Number missing & 0 & & 0 \\
Median & 6.8 & 6.8 & 6.8 \\
Mode & 6.8 & 6.8 & 6.8 \\
\hline
\end{tabular}


Table A-33. Sample statistics for Change in Axial AP R, showing Edgewise sample alone $(n=31)$, MARA+Edgewise alone $(n=33)$ and the total sample $(n=64)$.

\begin{tabular}{lccc}
\hline \multicolumn{1}{c}{ Statistic } & $\begin{array}{c}\text { Edgewise } \\
\text { Alone }\end{array}$ & $\begin{array}{c}\text { MARA+Edge } \\
\text { Alone }\end{array}$ & $\begin{array}{c}\text { Total } \\
\text { Sample }\end{array}$ \\
\hline Mean & -0.04 & -0.42 & -0.23 \\
Standard deviation & 0.80 & 0.89 & 0.86 \\
Standard error of mean & 0.14 & 0.16 & 0.11 \\
Upper 95\% Mean & 0.26 & -0.10 & -0.02 \\
Lower 95\% Mean & -0.33 & -0.73 & -0.45 \\
Sample size & 31 & 33 & 64 \\
Sample variance & 0.64 & 0.80 & 0.75 \\
Skewness (g1) & -0.69 & -0.98 & -0.85 \\
Kurtosis (g2) & 0.76 & 1.01 & 0.95 \\
Coef Variation & $-2,071.70$ & -214.87 & -371.08 \\
Number missing & 0 & 0 & 0 \\
Median & 0.0 & -0.2 & -0.1 \\
Mode & -0.1 & -0.2 & -0.1 \\
& & One-value (two tail) & 0.0349 \\
& & & 2.16 \\
& & &
\end{tabular}


Table A-34. Sample statistics for Axial MLCP Left Initial, showing Edgewise sample alone $(n=31)$, MARA + Edgewise alone $(n=33)$ and the total sample $(n=$ 64).

\begin{tabular}{lccc}
\hline \multicolumn{1}{c}{ Statistic } & $\begin{array}{c}\text { Edgewise } \\
\text { Alone }\end{array}$ & $\begin{array}{c}\text { MARA+Edge } \\
\text { Alone }\end{array}$ & $\begin{array}{c}\text { Total } \\
\text { Sample }\end{array}$ \\
\hline Mean & 46.16 & 46.15 & 46.16 \\
Standard deviation & 2.90 & 2.29 & 2.58 \\
Standard error of mean & 0.52 & 0.40 & 0.32 \\
Upper 95\% Mean & 47.23 & 46.96 & 46.80 \\
Lower 95\% Mean & 45.10 & 45.34 & 45.51 \\
Sample size & 31 & 33 & 64 \\
Sample variance & 8.39 & 5.24 & 6.66 \\
Skewness (g1) & -0.13 & 0.26 & 0.01 \\
Kurtosis (g2) & -0.07 & 0.12 & 0.05 \\
Coef Variation & 6.27 & 4.96 & 5.59 \\
Number missing & 0 & 0 & 0 \\
Median & 46.8 & 45.6 & 46.4 \\
Mode & 46.7 & 45.3 & 45.3 \\
\hline
\end{tabular}


Table A-35. Sample statistics for Axial MLCP Left Final, showing Edgewise sample alone $(n=31)$, MARA+Edgewise alone $(n=33)$ and the total sample $(n=$ 64).

\begin{tabular}{lccc}
\hline \multicolumn{1}{c}{ Statistic } & $\begin{array}{c}\text { Edgewise } \\
\text { Alone }\end{array}$ & $\begin{array}{c}\text { MARA+Edge } \\
\text { Alone }\end{array}$ & $\begin{array}{c}\text { Total } \\
\text { Sample }\end{array}$ \\
\hline Mean & 47.46 & 47.64 & 47.55 \\
Standard deviation & 3.12 & 2.51 & 2.80 \\
Standard error of mean & 0.56 & 0.44 & 0.35 \\
Upper 95\% Mean & 48.61 & 48.53 & 48.25 \\
Lower 95\% Mean & 46.32 & 46.75 & 46.85 \\
Sample size & 31 & 33 & 64 \\
Sample variance & 9.76 & 6.29 & 7.85 \\
Skewness (g1) & -0.22 & 0.38 & -0.03 \\
Kurtosis (g2) & 0.05 & 0.91 & 0.38 \\
Coef Variation & 6.58 & 5.27 & 5.89 \\
Number missing & 0 & 0 & 0 \\
Median & 47.6 & 47.3 & 47.5 \\
Mode & 47.1 & 45.3 & 47.1 \\
\hline
\end{tabular}


Table A-36. Sample statistics for Ch Axial MLCP L, showing Edgewise sample alone $(n=31)$, MARA+Edgewise alone $(n=33)$ and the total sample $(n=64)$.

\begin{tabular}{lccc}
\hline \multicolumn{1}{c}{ Statistic } & $\begin{array}{c}\text { Edgewise } \\
\text { Alone }\end{array}$ & $\begin{array}{c}\text { MARA+Edge } \\
\text { Alone }\end{array}$ & $\begin{array}{c}\text { Total } \\
\text { Sample }\end{array}$ \\
\hline Mean & 1.30 & 1.49 & 1.40 \\
Standard deviation & 1.13 & 1.16 & 1.14 \\
Standard error of mean & 0.20 & 0.20 & 0.14 \\
Upper 95\% Mean & 1.71 & 1.90 & 1.68 \\
Lower 95\% Mean & 0.88 & 1.08 & 1.11 \\
Sample size & 31 & 33 & 64 \\
Sample variance & 1.28 & 1.34 & 1.30 \\
Skewness (g1) & 0.50 & 0.48 & 0.48 \\
Kurtosis (g2) & 0.39 & -0.75 & -0.30 \\
Coef Variation & 87.23 & 77.65 & 81.61 \\
Number missing & 0 & 0 & 0 \\
Median & 1.1 & 1.3 & 1.2 \\
Mode & 0.9 & 2.3 & 0.7 \\
& & One-sample t-test & 9.80 \\
& & & $<0.0001$ \\
\hline
\end{tabular}


Table A-37. Sample statistics for Axial MLCP Right Initial, showing Edgewise sample alone $(n=31)$, MARA+Edgewise alone $(n=33)$ and the total sample $(n=$ 64).

\begin{tabular}{lccc}
\hline \multicolumn{1}{c}{ Statistic } & $\begin{array}{c}\text { Edgewise } \\
\text { Alone }\end{array}$ & $\begin{array}{c}\text { MARA+Edge } \\
\text { Alone }\end{array}$ & $\begin{array}{c}\text { Total } \\
\text { Sample }\end{array}$ \\
\hline Mean & 46.62 & 46.15 & 46.38 \\
Standard deviation & 2.99 & 2.61 & 2.79 \\
Standard error of mean & 0.54 & 0.45 & 0.35 \\
Upper 95\% Mean & 47.72 & 47.08 & 47.08 \\
Lower 95\% Mean & 45.53 & 45.23 & 45.68 \\
Sample size & 31 & 33 & 64 \\
Sample variance & 8.95 & 6.80 & 7.77 \\
Skewness (g1) & -0.01 & 0.47 & 0.22 \\
Kurtosis (g2) & -0.19 & 0.20 & -0.15 \\
Coef Variation & 6.42 & 5.65 & 6.01 \\
Number missing & 0 & & 0 \\
Median & 47.0 & 46.0 & 46.3 \\
Mode & 47.0 & 45.4 & 47.0 \\
\hline
\end{tabular}


Table A-38. Sample statistics for Axial MLCP Right Final, showing Edgewise sample alone $(n=31)$, MARA+Edgewise alone $(n=33)$ and the total sample $(n=$ 64).

\begin{tabular}{lccc}
\hline \multicolumn{1}{c}{ Statistic } & $\begin{array}{c}\text { Edgewise } \\
\text { Alone }\end{array}$ & $\begin{array}{c}\text { MARA+Edge } \\
\text { Alone }\end{array}$ & $\begin{array}{c}\text { Total } \\
\text { Sample }\end{array}$ \\
\hline Mean & 47.94 & 47.62 & 47.78 \\
Standard deviation & 2.95 & 2.80 & 2.86 \\
Standard error of mean & 0.53 & 0.49 & 0.36 \\
Upper 95\% Mean & 49.02 & 48.62 & 48.49 \\
Lower 95\% Mean & 46.86 & 46.63 & 47.06 \\
Sample size & 31 & 33 & 64 \\
Sample variance & 8.73 & 7.85 & 8.17 \\
Skewness (g1) & 0.04 & 1.28 & 0.62 \\
Kurtosis (g2) & -0.05 & 2.18 & 0.69 \\
Coef Variation & 6.16 & 5.88 & 5.98 \\
Number missing & 0 & 0 & 0 \\
Median & 48.1 & 47.3 & 47.4 \\
Mode & 48.9 & 45.6 & 45.9 \\
\hline
\end{tabular}


Table A-39. Sample statistics for Change in Axial MLCP R, showing Edgewise sample alone $(n=31)$, MARA + Edgewise alone $(n=33)$ and the total sample $(n=$ 64).

\begin{tabular}{lccc}
\hline \multicolumn{1}{c}{ Statistic } & $\begin{array}{c}\text { Edgewise } \\
\text { Alone }\end{array}$ & $\begin{array}{c}\text { MARA+Edge } \\
\text { Alone }\end{array}$ & $\begin{array}{c}\text { Total } \\
\text { Sample }\end{array}$ \\
\hline Mean & 1.32 & 1.47 & 1.40 \\
Standard deviation & 1.18 & 1.11 & 1.14 \\
Standard error of mean & 0.21 & 0.19 & 0.14 \\
Upper 95\% Mean & 1.75 & 1.86 & 1.68 \\
Lower 95\% Mean & 0.88 & 1.08 & 1.11 \\
Sample size & 31 & 33 & 64 \\
Sample variance & 1.40 & 1.22 & 1.29 \\
Skewness (g1) & 0.94 & 0.17 & 0.55 \\
Kurtosis (g2) & 0.33 & -0.36 & -0.19 \\
Coef Variation & 89.90 & 75.10 & 81.44 \\
Number missing & 0 & 0 & 0 \\
Median & 1.0 & 1.6 & 1.3 \\
Mode & 1.3 & 1.0 & 1.3 \\
& & One-sample t-test & 9.82 \\
& & & $<0.0001$ \\
\hline
\end{tabular}


Table A-40. Sample statistics for Axial APCD Initial, showing Edgewise sample alone $(n=31)$, MARA+Edgewise alone $(n=33)$ and the total sample $(n=64)$.

\begin{tabular}{lccc}
\hline \multicolumn{1}{c}{ Statistic } & $\begin{array}{c}\text { Edgewise } \\
\text { Alone }\end{array}$ & $\begin{array}{c}\text { MARA+Edge } \\
\text { Alone }\end{array}$ & $\begin{array}{c}\text { Total } \\
\text { Sample }\end{array}$ \\
\hline Mean & 0.31 & 0.09 & 0.20 \\
Standard deviation & 1.62 & 0.84 & 1.27 \\
Standard error of mean & 0.29 & 0.15 & 0.16 \\
Upper 95\% Mean & 0.90 & 0.39 & 0.51 \\
Lower 95\% Mean & -0.29 & -0.21 & -0.12 \\
Sample size & 31 & 33 & 64 \\
Sample variance & 2.61 & 0.71 & 1.62 \\
Skewness (g1) & 0.15 & -0.03 & 0.28 \\
Kurtosis (g2) & -0.96 & 0.21 & -0.09 \\
Coef Variation & 527.38 & 899.32 & 646.05 \\
Number missing & 0 & 0 & 0 \\
Median & 0.0 & 0.0 & 0.0 \\
Mode & 0.0 & 0.0 & 0.0 \\
\hline
\end{tabular}


Table A-41. Sample statistics for Axial APCD Final, showing Edgewise sample alone $(n=31)$, MARA+Edgewise alone $(n=33)$ and the total sample $(n=64)$.

\begin{tabular}{lccc}
\hline \multicolumn{1}{c}{ Statistic } & $\begin{array}{c}\text { Edgewise } \\
\text { Alone }\end{array}$ & $\begin{array}{c}\text { MARA+Edge } \\
\text { Alone }\end{array}$ & $\begin{array}{c}\text { Total } \\
\text { Sample }\end{array}$ \\
\hline Mean & 0.01 & -0.14 & -0.06 \\
Standard deviation & 1.29 & 1.01 & 1.14 \\
Standard error of mean & 0.23 & 0.18 & 0.14 \\
Upper 95\% Mean & 0.48 & 0.22 & 0.22 \\
Lower 95\% Mean & -0.46 & -0.49 & -0.35 \\
Sample size & 31 & 33 & 64 \\
Sample variance & 1.65 & 1.02 & 1.31 \\
Skewness (g1) & -0.47 & -0.57 & -0.45 \\
Kurtosis (g2) & 0.16 & 0.70 & 0.35 \\
Coef Variation & $9,966.60$ & -739.45 & $-1,786.37$ \\
Number missing & 0 & 0 & 0 \\
Median & 0.0 & 0.0 & 0.0 \\
Mode & 0.0 & 0.0 & 0.0 \\
\hline
\end{tabular}


Table A-42. Sample statistics for Change in Axial APCD, showing Edgewise sample alone $(n=31)$, MARA + Edgewise alone $(n=33)$ and the total sample $(n=$ 64).

\begin{tabular}{lccc}
\hline \multicolumn{1}{c}{ Statistic } & $\begin{array}{c}\text { Edgewise } \\
\text { Alone }\end{array}$ & $\begin{array}{c}\text { MARA+Edge } \\
\text { Alone }\end{array}$ & $\begin{array}{c}\text { Total } \\
\text { Sample }\end{array}$ \\
\hline Mean & -0.29 & -0.23 & -0.26 \\
Standard deviation & 1.28 & 0.99 & 1.13 \\
Standard error of mean & 0.23 & 0.17 & 0.14 \\
Upper 95\% Mean & 0.18 & 0.12 & 0.02 \\
Lower 95\% Mean & -0.76 & -0.58 & -0.54 \\
Sample size & 31 & 33 & 64 \\
Sample variance & 1.64 & 0.98 & 1.28 \\
Skewness (g1) & -0.78 & -0.33 & -0.65 \\
Kurtosis (g2) & 0.24 & 0.10 & 0.34 \\
Coef Variation & -436.61 & -430.32 & -433.93 \\
Number missing & 0 & 0 & 0 \\
Median & 0.0 & 0.0 & 0.0 \\
Mode & 0.0 & 0.0 & 0.0 \\
& & One-sample t-test & 1.84 \\
& & & 0.0699 \\
\hline
\end{tabular}


Table A-43. Sample statistics for Axial CA Left Initial, showing Edgewise sample alone $(n=31)$, MARA+Edgewise alone $(n=33)$ and the total sample $(n=64)$.

\begin{tabular}{lccc}
\hline \multicolumn{1}{c}{ Statistic } & $\begin{array}{c}\text { Edgewise } \\
\text { Alone }\end{array}$ & $\begin{array}{c}\text { MARA+Edge } \\
\text { Alone }\end{array}$ & $\begin{array}{c}\text { Total } \\
\text { Sample }\end{array}$ \\
\hline Mean & 63.41 & 61.65 & 62.50 \\
Standard deviation & 8.32 & 5.69 & 7.08 \\
Standard error of mean & 1.49 & 0.99 & 0.89 \\
Upper 95\% Mean & 66.46 & 63.67 & 64.27 \\
Lower 95\% Mean & 60.36 & 59.64 & 60.74 \\
Sample size & 31 & 33 & 64 \\
Sample variance & 69.15 & 32.33 & 50.13 \\
Skewness (g1) & 0.78 & -0.62 & 0.56 \\
Kurtosis (g2) & 2.45 & 0.42 & 2.78 \\
Coef Variation & 13.11 & 9.22 & 11.33 \\
Number missing & 0 & 0 & 0 \\
Median & 62.4 & 62.7 & 62.6 \\
Mode & 61.6 & 65.9 & 61.6 \\
\hline
\end{tabular}


Table A-44. Sample statistics for Axial CA Left Final, showing Edgewise sample alone $(n=31)$, MARA+Edgewise alone $(n=33)$ and the total sample $(n=64)$.

\begin{tabular}{lccc}
\hline \multicolumn{1}{c}{ Statistic } & $\begin{array}{c}\text { Edgewise } \\
\text { Alone }\end{array}$ & $\begin{array}{c}\text { MARA+Edge } \\
\text { Alone }\end{array}$ & $\begin{array}{c}\text { Total } \\
\text { Sample }\end{array}$ \\
\hline Mean & 64.45 & 62.91 & 63.65 \\
Standard deviation & 8.02 & 5.55 & 6.85 \\
Standard error of mean & 1.44 & 0.97 & 0.86 \\
Upper 95\% Mean & 67.39 & 64.87 & 65.36 \\
Lower 95\% Mean & 61.51 & 60.94 & 61.94 \\
Sample size & 31 & 33 & 64 \\
Sample variance & 64.35 & 30.75 & 46.87 \\
Skewness (g1) & 0.75 & -0.86 & 0.45 \\
Kurtosis (g2) & 2.36 & 0.04 & 2.57 \\
Coef Variation & 12.45 & 8.82 & 10.76 \\
Number missing & 0 & 0 & 0 \\
Median & 65.3 & 63.9 & 65.0 \\
Mode & 59.5 & 63.4 & 63.4 \\
\hline
\end{tabular}


Table A-45. Sample statistics for Change in Axial CA $L$, showing Edgewise sample alone $(n=31)$, MARA+Edgewise alone $(n=33)$ and the total sample $(n=64)$.

\begin{tabular}{lccc}
\hline \multicolumn{1}{c}{ Statistic } & $\begin{array}{c}\text { Edgewise } \\
\text { Alone }\end{array}$ & $\begin{array}{c}\text { MARA+Edge } \\
\text { Alone }\end{array}$ & $\begin{array}{c}\text { Total } \\
\text { Sample }\end{array}$ \\
\hline Mean & 1.04 & 1.25 & 1.15 \\
Standard deviation & 4.52 & 4.20 & 4.32 \\
Standard error of mean & 0.81 & 0.73 & 0.54 \\
Upper 95\% Mean & 2.69 & 2.74 & 2.23 \\
Lower 95\% Mean & -0.62 & -0.24 & 0.07 \\
Sample size & 31 & 33 & 64 \\
Sample variance & 20.39 & 17.61 & 18.66 \\
Skewness (g1) & -0.33 & -0.84 & -0.56 \\
Kurtosis (g2) & -0.23 & 2.56 & 0.83 \\
Coef Variation & 434.72 & 335.27 & 376.17 \\
Number missing & 0 & 0 & 0 \\
Median & 1.6 & 1.9 & 1.7 \\
Mode &. & 1.2 & 3.6 \\
& & One-sample t-test & 2.13 \\
& & & 0.0374 \\
\hline
\end{tabular}


Table A-46. Sample statistics for Axial CA Right Initial, showing Edgewise sample alone $(n=31)$, MARA+Edgewise alone $(n=33)$ and the total sample $(n=64)$.

\begin{tabular}{lccc}
\hline \multicolumn{1}{c}{ Statistic } & $\begin{array}{c}\text { Edgewise } \\
\text { Alone }\end{array}$ & $\begin{array}{c}\text { MARA+Edge } \\
\text { Alone }\end{array}$ & $\begin{array}{c}\text { Total } \\
\text { Sample }\end{array}$ \\
\hline Mean & 63.18 & 62.03 & 62.59 \\
Standard deviation & 6.77 & 6.75 & 6.73 \\
Standard error of mean & 1.22 & 1.18 & 0.84 \\
Upper 95\% Mean & 65.66 & 64.43 & 64.27 \\
Lower 95\% Mean & 60.70 & 59.64 & 60.91 \\
Sample size & 31 & 33 & 64 \\
Sample variance & 45.81 & 45.61 & 45.32 \\
Skewness (g1) & 0.25 & -0.78 & -0.27 \\
Kurtosis (g2) & 0.08 & 1.70 & 0.92 \\
Coef Variation & 10.71 & 10.89 & 10.76 \\
Number missing & 0 & 0 & 0 \\
Median & 63.1 & 62.3 & 62.7 \\
Mode & 54.3 & 55.1 & 55.1 \\
\hline
\end{tabular}


Table A-47. Sample statistics for Axial CA Right Final, showing Edgewise sample alone $(n=31)$, MARA+Edgewise alone $(n=33)$ and the total sample $(n=64)$.

\begin{tabular}{lccc}
\hline \multicolumn{1}{c}{ Statistic } & $\begin{array}{c}\text { Edgewise } \\
\text { Alone }\end{array}$ & $\begin{array}{c}\text { MARA+Edge } \\
\text { Alone }\end{array}$ & $\begin{array}{c}\text { Total } \\
\text { Sample }\end{array}$ \\
\hline Mean & 63.40 & 62.63 & 63.00 \\
Standard deviation & 5.43 & 6.70 & 6.08 \\
Standard error of mean & 0.98 & 1.17 & 0.76 \\
Upper 95\% Mean & 65.39 & 65.01 & 64.52 \\
Lower 95\% Mean & 61.41 & 60.26 & 61.49 \\
Sample size & 31 & 33 & 64 \\
Sample variance & 29.49 & 44.92 & 37.01 \\
Skewness (g1) & -0.20 & -0.02 & -0.12 \\
Kurtosis (g2) & 1.14 & 0.08 & 0.39 \\
Coef Variation & 8.57 & 10.70 & 9.66 \\
Number missing & 0 & & 0 \\
Median & 63.3 & 61.7 & 62.9 \\
Mode & 62.7 & 59.9 & 62.7 \\
\hline
\end{tabular}


Table A-48. Sample statistics for Change in Axial CA R, showing Edgewise sample alone $(n=31)$, MARA+Edgewise alone $(n=33)$ and the total sample $(n=64)$.

\begin{tabular}{lccc}
\hline \multicolumn{1}{c}{ Statistic } & $\begin{array}{c}\text { Edgewise } \\
\text { Alone }\end{array}$ & $\begin{array}{c}\text { MARA+Edge } \\
\text { Alone }\end{array}$ & $\begin{array}{c}\text { Total } \\
\text { Sample }\end{array}$ \\
\hline Mean & 0.22 & 0.60 & 0.42 \\
Standard deviation & 4.23 & 4.93 & 4.57 \\
Standard error of mean & 0.76 & 0.86 & 0.57 \\
Upper 95\% Mean & 1.77 & 2.35 & 1.56 \\
Lower 95\% Mean & -1.33 & -1.15 & -0.73 \\
Sample size & 31 & 33 & 64 \\
Sample variance & 17.85 & 24.31 & 20.88 \\
Skewness (g1) & -0.31 & -1.31 & -0.90 \\
Kurtosis (g2) & -0.53 & 2.45 & 1.23 \\
Coef Variation & $1,926.12$ & 821.70 & $1,099.51$ \\
Number missing & 0 & 0 & 0 \\
Median & 0.7 & 1.9 & 1.3 \\
Mode & 2.2 & -1.4 & 2.2 \\
& & One-value (two tail) & 0.4696 \\
& & & 0.73 \\
& & &
\end{tabular}


Table A-49. Sample statistics for Axial LACP Left Initial, showing Edgewise sample alone $(n=31)$, MARA+Edgewise alone $(n=33)$ and the total sample $(n=$ 64).

\begin{tabular}{lccc}
\hline \multicolumn{1}{c}{ Statistic } & $\begin{array}{c}\text { Edgewise } \\
\text { Alone }\end{array}$ & $\begin{array}{c}\text { MARA+Edge } \\
\text { Alone }\end{array}$ & $\begin{array}{c}\text { Total } \\
\text { Sample }\end{array}$ \\
\hline Mean & 100.63 & 101.49 & 101.07 \\
Standard deviation & 17.53 & 21.53 & 19.54 \\
Standard error of mean & 3.15 & 3.75 & 2.44 \\
Upper 95\% Mean & 107.06 & 109.12 & 105.95 \\
Lower 95\% Mean & 94.20 & 93.85 & 96.19 \\
Sample size & 31 & 33 & 64 \\
Sample variance & 307.27 & 463.39 & 381.88 \\
Skewness (g1) & -0.29 & 0.21 & 0.06 \\
Kurtosis (g2) & -0.14 & -0.99 & -0.66 \\
Coef Variation & 17.42 & 21.21 & 19.33 \\
Number missing & 0 & 0 & 0 \\
Median & 101.0 & 99.5 & 100.1 \\
Mode &. & 99.5 & 99.5 \\
\hline
\end{tabular}


Table A-50. Sample statistics for Axial LACP Left Final, showing Edgewise sample alone $(n=31)$, MARA + Edgewise alone $(n=33)$ and the total sample $(n=$ 64).

\begin{tabular}{lccc}
\hline \multicolumn{1}{c}{ Statistic } & $\begin{array}{c}\text { Edgewise } \\
\text { Alone }\end{array}$ & $\begin{array}{c}\text { MARA+Edge } \\
\text { Alone }\end{array}$ & $\begin{array}{c}\text { Total } \\
\text { Sample }\end{array}$ \\
\hline Mean & 107.17 & 105.42 & 106.27 \\
Standard deviation & 16.25 & 23.35 & 20.08 \\
Standard error of mean & 2.92 & 4.06 & 2.51 \\
Upper 95\% Mean & 113.13 & 113.70 & 111.29 \\
Lower 95\% Mean & 101.21 & 97.15 & 101.25 \\
Sample size & 31 & 33 & 64 \\
Sample variance & 264.04 & 545.01 & 403.33 \\
Skewness (g1) & -0.03 & -0.22 & -0.22 \\
Kurtosis (g2) & -0.45 & -0.92 & -0.57 \\
Coef Variation & 15.16 & 22.14 & 18.90 \\
Number missing & 0 & 0 & 0 \\
Median & 105.3 & 107.5 & 106.8 \\
Mode & 100.3 & 75.5 & 100.3 \\
\hline
\end{tabular}


Table A-51. Sample statistics for Change in Axial LACP L, showing Edgewise sample alone $(n=31)$, MARA + Edgewise alone $(n=33)$ and the total sample $(n=$ 64).

\begin{tabular}{lccc}
\hline \multicolumn{1}{c}{ Statistic } & $\begin{array}{c}\text { Edgewise } \\
\text { Alone }\end{array}$ & $\begin{array}{c}\text { MARA+Edge } \\
\text { Alone }\end{array}$ & $\begin{array}{c}\text { Total } \\
\text { Sample }\end{array}$ \\
Mean & & & \\
Standard deviation & 13.46 & 3.94 & 5.20 \\
Standard error of mean & 2.42 & 11.65 & 12.53 \\
Upper 95\% Mean & 11.47 & 2.03 & 1.57 \\
Lower 95\% Mean & 1.60 & 8.07 & 8.32 \\
Sample size & 31.00 & -0.19 & 2.07 \\
Sample variance & 181.30 & 33.00 & 64.00 \\
Skewness (g1) & 0.02 & 135.63 & 156.94 \\
Kurtosis (g2) & -0.52 & 0.17 & 0.12 \\
Coef Variation & 206.03 & -0.08 & -0.38 \\
Number missing & 0.00 & 295.85 & 241.13 \\
Median & 7.20 & 0.00 & 0.00 \\
Mode &. & 4.70 & 5.50 \\
& & & -0.50 \\
& & One-sample t-test & 3.32 \\
& & & 0.0015 \\
\hline
\end{tabular}


Table A-52. Sample statistics for Axial LACP Right Initial, showing Edgewise sample alone $(n=31)$, MARA + Edgewise alone $(n=33)$ and the total sample $(n=$ 64).

\begin{tabular}{lccc}
\hline \multicolumn{1}{c}{ Statistic } & $\begin{array}{c}\text { Edgewise } \\
\text { Alone }\end{array}$ & $\begin{array}{c}\text { MARA+Edge } \\
\text { Alone }\end{array}$ & $\begin{array}{c}\text { Total } \\
\text { Sample }\end{array}$ \\
\hline Mean & 103.63 & 104.49 & 104.08 \\
Standard deviation & 16.76 & 24.65 & 21.04 \\
Standard error of mean & 3.01 & 4.29 & 2.63 \\
Upper 95\% Mean & 109.78 & 113.23 & 109.33 \\
Lower 95\% Mean & 97.48 & 95.76 & 98.82 \\
Sample size & 31 & 33 & 64 \\
Sample variance & 280.98 & 607.40 & 442.51 \\
Skewness (g1) & 0.30 & 1.04 & 0.92 \\
Kurtosis (g2) & 0.40 & 1.37 & 1.64 \\
Coef Variation & 16.18 & 23.59 & 20.21 \\
Number missing & 0 & 0 & 0 \\
Median & 105.7 & 100.8 & 102.7 \\
Mode &. & 100.8 & 95.1 \\
\hline
\end{tabular}


Table A-53. Sample statistics for Axial LACP Right Final, showing Edgewise sample alone $(n=31)$, MARA + Edgewise alone $(n=33)$ and the total sample $(n=$ 64).

\begin{tabular}{lccc}
\hline \multicolumn{1}{c}{ Statistic } & $\begin{array}{c}\text { Edgewise } \\
\text { Alone }\end{array}$ & $\begin{array}{c}\text { MARA+Edge } \\
\text { Alone }\end{array}$ & $\begin{array}{c}\text { Total } \\
\text { Sample }\end{array}$ \\
\hline Mean & 106.80 & 109.88 & 108.39 \\
Standard deviation & 16.67 & 26.03 & 21.89 \\
Standard error of mean & 2.99 & 4.53 & 2.74 \\
Upper 95\% Mean & 112.91 & 119.11 & 113.85 \\
Lower 95\% Mean & 100.69 & 100.64 & 102.92 \\
Sample size & 31 & 33 & 64 \\
Sample variance & 277.91 & 677.72 & 478.98 \\
Skewness (g1) & -0.21 & -0.47 & -0.34 \\
Kurtosis (g2) & -0.52 & -0.64 & -0.34 \\
Coef Variation & 15.61 & 23.69 & 20.19 \\
Number missing & 0 & 0 & 0 \\
Median & 107.6 & 112.9 & 110.1 \\
Mode &. &. &. \\
\hline
\end{tabular}


Table A-54. Sample statistics for Change in Axial LACP R, showing Edgewise sample alone $(n=31)$, MARA + Edgewise alone $(n=33)$ and the total sample $(n=$ 64).

\begin{tabular}{lccc}
\hline \multicolumn{1}{c}{ Statistic } & $\begin{array}{c}\text { Edgewise } \\
\text { Alone }\end{array}$ & $\begin{array}{c}\text { MARA+Edge } \\
\text { Alone }\end{array}$ & $\begin{array}{c}\text { Total } \\
\text { Sample }\end{array}$ \\
\hline Mean & 3.17 & 5.38 & 4.31 \\
Standard deviation & 14.03 & 16.18 & 15.10 \\
Standard error of mean & 2.52 & 2.82 & 1.89 \\
Upper 95\% Mean & 8.32 & 11.12 & 8.08 \\
Lower 95\% Mean & -1.98 & -0.35 & 0.54 \\
Sample size & 31 & 33 & 64 \\
Sample variance & 196.96 & 261.76 & 227.99 \\
Skewness (g1) & -0.20 & -0.63 & -0.42 \\
Kurtosis (g2) & 0.21 & 0.29 & 0.12 \\
Coef Variation & 443.04 & 300.62 & 350.38 \\
Number missing & 0 & 0 & 0 \\
Median & 2.2 & 5.8 & 3.4 \\
Mode & & & -32.7 \\
& & One-sample t-test & 2.28 \\
& & & 0.0258 \\
\hline
\end{tabular}


Table A-55. Sample statistics for Sagittal AJS Left Initial, showing Edgewise sample alone $(n=31)$, MARA + Edgewise alone $(n=33)$ and the total sample $(n=$ 64).

\begin{tabular}{lccc}
\hline \multicolumn{1}{c}{ Statistic } & $\begin{array}{c}\text { Edgewise } \\
\text { Alone }\end{array}$ & $\begin{array}{c}\text { MARA+Edge } \\
\text { Alone }\end{array}$ & $\begin{array}{c}\text { Total } \\
\text { Sample }\end{array}$ \\
\hline Mean & 1.26 & 1.42 & 1.35 \\
Standard deviation & 0.51 & 0.71 & 0.62 \\
Standard error of mean & 0.09 & 0.12 & 0.08 \\
Upper 95\% Mean & 1.45 & 1.68 & 1.50 \\
Lower 95\% Mean & 1.07 & 1.17 & 1.19 \\
Sample size & 31 & 33 & 64 \\
Sample variance & 0.26 & 0.50 & 0.39 \\
Skewness (g1) & 0.77 & 1.22 & 1.21 \\
Kurtosis (g2) & 0.23 & 1.73 & 1.94 \\
Coef Variation & 40.77 & 49.74 & 46.28 \\
Number missing & 0 & 0 & 0 \\
Median & 1.2 & 1.3 & 1.3 \\
Mode & 1.0 & 1.3 & 1.0 \\
\hline
\end{tabular}


Table A-56. Sample statistics for Sagittal AJS Left Final, showing Edgewise sample alone $(n=31)$, MARA + Edgewise alone $(n=33)$ and the total sample $(n=$ 64).

\begin{tabular}{lccc}
\hline \multicolumn{1}{c}{ Statistic } & $\begin{array}{c}\text { Edgewise } \\
\text { Alone }\end{array}$ & $\begin{array}{c}\text { MARA+Edge } \\
\text { Alone }\end{array}$ & $\begin{array}{c}\text { Total } \\
\text { Sample }\end{array}$ \\
\hline Mean & 1.19 & 1.29 & 1.24 \\
Standard deviation & 0.44 & 0.56 & 0.50 \\
Standard error of mean & 0.08 & 0.10 & 0.06 \\
Upper 95\% Mean & 1.35 & 1.49 & 1.37 \\
Lower 95\% Mean & 1.03 & 1.09 & 1.12 \\
Sample size & 31 & 33 & 64 \\
Sample variance & 0.19 & 0.32 & 0.25 \\
Skewness (g1) & 1.09 & 0.56 & 0.78 \\
Kurtosis (g2) & 2.35 & -0.52 & 0.30 \\
Coef Variation & 36.58 & 43.66 & 40.59 \\
Number missing & 0 & 0 & 0 \\
Median & 1.2 & 1.1 & 1.2 \\
Mode & 1.2 & 1.1 & 0.8 \\
\hline
\end{tabular}


Table A-57. Sample statistics for Change in Sagittal AJS L, showing Edgewise sample alone $(n=31)$, MARA + Edgewise alone $(n=33)$ and the total sample $(n=$ 64).

\begin{tabular}{lccc}
\hline \multicolumn{1}{c}{ Statistic } & $\begin{array}{c}\text { Edgewise } \\
\text { Alone }\end{array}$ & $\begin{array}{c}\text { MARA+Edge } \\
\text { Alone }\end{array}$ & $\begin{array}{c}\text { Total } \\
\text { Sample }\end{array}$ \\
\hline Mean & -0.07 & -0.13 & -0.10 \\
Standard deviation & 0.56 & 0.59 & 0.57 \\
Standard error of mean & 0.10 & 0.10 & 0.07 \\
Upper 95\% Mean & 0.14 & 0.08 & 0.04 \\
Lower 95\% Mean & -0.28 & -0.34 & -0.25 \\
Sample size & 31 & 33 & 64 \\
Sample variance & 0.32 & 0.35 & 0.33 \\
Skewness (g1) & -0.44 & 0.23 & -0.08 \\
Kurtosis (g2) & -0.08 & -0.38 & -0.39 \\
Coef Variation & -795.60 & -442.38 & -556.64 \\
Number missing & 0 & 0 & 0 \\
Median & -0.1 & -0.2 & -0.1 \\
Mode & -0.1 & -0.5 & -0.2 \\
& & One-sample t-test & 1.44 \\
& & & 0.1556 \\
\hline
\end{tabular}


Table A-58. Sample statistics for Sagittal MJS Left Initial, showing Edgewise sample alone $(n=31)$, MARA+Edgewise alone $(n=33)$ and the total sample $(n=$ 64).

\begin{tabular}{lccc}
\hline \multicolumn{1}{c}{ Statistic } & $\begin{array}{c}\text { Edgewise } \\
\text { Alone }\end{array}$ & $\begin{array}{c}\text { MARA+Edge } \\
\text { Alone }\end{array}$ & $\begin{array}{c}\text { Total } \\
\text { Sample }\end{array}$ \\
\hline Mean & 2.00 & 2.63 & 2.33 \\
Standard deviation & 0.64 & 0.74 & 0.76 \\
Standard error of mean & 0.11 & 0.13 & 0.09 \\
Upper 95\% Mean & 2.23 & 2.89 & 2.51 \\
Lower 95\% Mean & 1.77 & 2.37 & 2.14 \\
Sample size & 31 & 33 & 64 \\
Sample variance & 0.40 & 0.55 & 0.57 \\
Skewness (g1) & 0.25 & 0.53 & 0.47 \\
Kurtosis (g2) & 0.33 & 0.05 & 0.33 \\
Coef Variation & 31.75 & 28.24 & 32.56 \\
Number missing & 0 & 0 & 0 \\
Median & 2.0 & 2.6 & 2.2 \\
Mode & 2.0 & 3.1 & 2.0 \\
\hline
\end{tabular}


Table A-59. Sample statistics for Sagittal MJS Left Final, showing Edgewise sample alone $(n=31)$, MARA+Edgewise alone $(n=33)$ and the total sample $(n=$ 64).

\begin{tabular}{lccc}
\hline \multicolumn{1}{c}{ Statistic } & $\begin{array}{c}\text { Edgewise } \\
\text { Alone }\end{array}$ & $\begin{array}{c}\text { MARA+Edge } \\
\text { Alone }\end{array}$ & $\begin{array}{c}\text { Total } \\
\text { Sample }\end{array}$ \\
\hline Mean & 2.24 & 2.50 & 2.37 \\
Standard deviation & 0.75 & 0.86 & 0.81 \\
Standard error of mean & 0.13 & 0.15 & 0.10 \\
Upper 95\% Mean & 2.51 & 2.81 & 2.58 \\
Lower 95\% Mean & 1.96 & 2.20 & 2.17 \\
Sample size & 31 & 33 & 64 \\
Sample variance & 0.56 & 0.74 & 0.66 \\
Skewness (g1) & 0.39 & -0.08 & 0.16 \\
Kurtosis (g2) & 0.36 & 0.36 & 0.16 \\
Coef Variation & 33.35 & 34.37 & 34.20 \\
Number missing & 0 & 0 & 0 \\
Median & 2.1 & 2.7 & 2.5 \\
Mode & 2.0 & 2.7 & 2.0 \\
\hline
\end{tabular}


Table A-60. Sample statistics for Change in Sagittal MJS L, showing Edgewise sample alone $(n=31)$, MARA + Edgewise alone $(n=33)$ and the total sample $(n=$ 64).

\begin{tabular}{lccc}
\hline \multicolumn{1}{c}{ Statistic } & $\begin{array}{c}\text { Edgewise } \\
\text { Alone }\end{array}$ & $\begin{array}{c}\text { MARA+Edge } \\
\text { Alone }\end{array}$ & $\begin{array}{c}\text { Total } \\
\text { Sample }\end{array}$ \\
\hline Mean & 0.24 & -0.13 & 0.05 \\
Standard deviation & 0.71 & 0.72 & 0.73 \\
Standard error of mean & 0.13 & 0.12 & 0.09 \\
Upper 95\% Mean & 0.50 & 0.13 & 0.23 \\
Lower 95\% Mean & -0.02 & -0.38 & -0.13 \\
Sample size & 31 & 33 & 64 \\
Sample variance & 0.50 & 0.51 & 0.53 \\
Skewness (g1) & 0.32 & 0.20 & 0.22 \\
Kurtosis (g2) & 1.14 & 0.12 & 0.45 \\
Coef Variation & 300.99 & -563.26 & $1,508.15$ \\
Number missing & 0 & 0 & 0 \\
Median & 0.2 & 0.0 & 0.0 \\
Mode & -0.2 & 0.0 & -0.2 \\
& & P-value (two tail) & 0.5977 \\
\hline
\end{tabular}


Table A-61. Sample statistics for Sagittal PJS Left Final, showing Edgewise sample alone $(n=31)$, MARA + Edgewise alone $(n=33)$ and the total sample $(n=$ 64).

\begin{tabular}{lccc}
\hline \multicolumn{1}{c}{ Statistic } & $\begin{array}{c}\text { Edgewise } \\
\text { Alone }\end{array}$ & $\begin{array}{c}\text { MARA+Edge } \\
\text { Alone }\end{array}$ & $\begin{array}{c}\text { Total } \\
\text { Sample }\end{array}$ \\
\hline Mean & 2.77 & 2.85 & 2.81 \\
Standard deviation & 1.23 & 0.87 & 1.05 \\
Standard error of mean & 0.22 & 0.15 & 0.13 \\
Upper 95\% Mean & 3.22 & 3.16 & 3.07 \\
Lower 95\% Mean & 2.32 & 2.54 & 2.55 \\
Sample size & 31 & 33 & 64 \\
Sample variance & 1.50 & 0.75 & 1.10 \\
Skewness (g1) & 0.82 & 0.33 & 0.66 \\
Kurtosis (g2) & 0.79 & -0.15 & 0.75 \\
Coef Variation & 44.21 & 30.38 & 37.25 \\
Number missing & 0 & 0 & 0 \\
Median & 2.7 & 2.8 & 2.8 \\
Mode & 2.4 & 2.5 & 3.0 \\
\hline
\end{tabular}


Table A-62. Sample statistics for Sagittal PJS Left Final 2, showing Edgewise sample alone $(n=31)$, MARA+Edgewise alone $(n=33)$ and the total sample $(n=$ 64).

\begin{tabular}{lccc}
\hline \multicolumn{1}{c}{ Statistic } & $\begin{array}{c}\text { Edgewise } \\
\text { Alone }\end{array}$ & $\begin{array}{c}\text { MARA+Edge } \\
\text { Alone }\end{array}$ & $\begin{array}{c}\text { Total } \\
\text { Sample }\end{array}$ \\
\hline Mean & 2.72 & 2.67 & 2.69 \\
Standard deviation & 1.36 & 0.98 & 1.17 \\
Standard error of mean & 0.24 & 0.17 & 0.15 \\
Upper 95\% Mean & 3.21 & 3.02 & 2.99 \\
Lower 95\% Mean & 2.22 & 2.32 & 2.40 \\
Sample size & 31 & 33 & 64 \\
Sample variance & 1.84 & 0.96 & 1.37 \\
Skewness (g1) & 0.47 & 0.42 & 0.48 \\
Kurtosis (g2) & -0.90 & -0.80 & -0.66 \\
Coef Variation & 49.99 & 36.73 & 43.42 \\
Number missing & 0 & 0 & 0 \\
Median & 2.4 & 2.5 & 2.5 \\
Mode & 1.1 & 1.4 & 1.4 \\
\hline
\end{tabular}


Table A-63. Sample statistics for Change in Sagittal PJS L, showing Edgewise sample alone $(n=31)$, MARA+Edgewise alone $(n=33)$ and the total sample $(n=$ 64).

\begin{tabular}{lccc}
\hline \multicolumn{1}{c}{ Statistic } & $\begin{array}{c}\text { Edgewise } \\
\text { Alone }\end{array}$ & $\begin{array}{c}\text { MARA+Edge } \\
\text { Alone }\end{array}$ & $\begin{array}{c}\text { Total } \\
\text { Sample }\end{array}$ \\
\hline Mean & -0.05 & -0.18 & -0.12 \\
Standard deviation & 0.78 & 0.81 & 0.79 \\
Standard error of mean & 0.14 & 0.14 & 0.10 \\
Upper 95\% Mean & 0.23 & 0.11 & 0.08 \\
Lower 95\% Mean & -0.34 & -0.47 & -0.32 \\
Sample size & 31 & 33 & 64 \\
Sample variance & 0.61 & 0.66 & 0.63 \\
Skewness (g1) & -0.23 & 0.47 & 0.14 \\
Kurtosis (g2) & -0.80 & 2.89 & 0.95 \\
Coef Variation & $-1,427.21$ & -455.20 & -669.47 \\
Number missing & 0 & 0 & 0 \\
Median & 0.0 & -0.3 & -0.2 \\
Mode & -0.5 & -0.3 & -0.3 \\
& & One-sample t-test & 1.20 \\
& & & 0.2366 \\
\hline
\end{tabular}


Table A-64. Sample statistics for Sagittal AJS Right Initial, showing Edgewise sample alone $(n=31)$, MARA + Edgewise alone $(n=33)$ and the total sample $(n=$ 64).

\begin{tabular}{lccc}
\hline \multicolumn{1}{c}{ Statistic } & $\begin{array}{c}\text { Edgewise } \\
\text { Alone }\end{array}$ & $\begin{array}{c}\text { MARA+Edge } \\
\text { Alone }\end{array}$ & $\begin{array}{c}\text { Total } \\
\text { Sample }\end{array}$ \\
\hline Mean & 1.35 & 1.46 & 1.41 \\
Standard deviation & 0.36 & 0.61 & 0.50 \\
Standard error of mean & 0.07 & 0.11 & 0.06 \\
Upper 95\% Mean & 1.49 & 1.68 & 1.53 \\
Lower 95\% Mean & 1.22 & 1.24 & 1.28 \\
Sample size & 31 & 33 & 64 \\
Sample variance & 0.13 & 0.37 & 0.25 \\
Skewness (g1) & -0.28 & 0.07 & 0.16 \\
Kurtosis (g2) & 0.30 & -1.07 & -0.41 \\
Coef Variation & 26.94 & 41.64 & 35.80 \\
Number missing & 0 & 0 & 0 \\
Median & 1.3 & 1.5 & 1.4 \\
Mode & 1.1 & 0.6 & 1.5 \\
\hline
\end{tabular}


Table A-65. Sample statistics for Sagittal AJS Right Final, showing Edgewise sample alone $(n=31)$, MARA+Edgewise alone $(n=33)$ and the total sample $(n=$ 64).

\begin{tabular}{lccc}
\hline \multicolumn{1}{c}{ Statistic } & $\begin{array}{c}\text { Edgewise } \\
\text { Alone }\end{array}$ & $\begin{array}{c}\text { MARA+Edge } \\
\text { Alone }\end{array}$ & $\begin{array}{c}\text { Total } \\
\text { Sample }\end{array}$ \\
\hline Mean & 1.35 & 1.51 & 1.43 \\
Standard deviation & 0.44 & 0.66 & 0.57 \\
Standard error of mean & 0.08 & 0.12 & 0.07 \\
Upper 95\% Mean & 1.52 & 1.74 & 1.58 \\
Lower 95\% Mean & 1.19 & 1.27 & 1.29 \\
Sample size & 31 & 33 & 64 \\
Sample variance & 0.19 & 0.44 & 0.32 \\
Skewness (g1) & 1.00 & 0.35 & 0.65 \\
Kurtosis (g2) & 2.48 & 0.01 & 0.76 \\
Coef Variation & 32.39 & 43.81 & 39.42 \\
Number missing & 0 & 0 & 0 \\
Median & 1.3 & 1.7 & 1.5 \\
Mode & 1.5 & 0.8 & 0.8 \\
\hline
\end{tabular}


Table A-66. Sample statistics for Change in Sagittal AJS R, showing Edgewise sample alone $(n=31)$, MARA+Edgewise alone $(n=33)$ and the total sample $(n=$ 64).

\begin{tabular}{lccc}
\hline \multicolumn{1}{c}{ Statistic } & $\begin{array}{c}\text { Edgewise } \\
\text { Alone }\end{array}$ & $\begin{array}{c}\text { MARA+Edge } \\
\text { Alone }\end{array}$ & $\begin{array}{c}\text { Total } \\
\text { Sample }\end{array}$ \\
Mean & 0.00 & 0.05 & 0.03 \\
Standard deviation & 0.49 & 0.55 & 0.51 \\
Standard error of mean & 0.09 & 0.10 & 0.06 \\
Upper 95\% Mean & 0.18 & 0.24 & 0.16 \\
Lower 95\% Mean & -0.18 & -0.15 & -0.10 \\
Sample size & 31 & 33 & 64 \\
Sample variance & 0.24 & 0.30 & 0.26 \\
Skewness (g1) & 0.33 & -0.23 & 0.01 \\
Kurtosis (g2) & 0.98 & 0.03 & 0.25 \\
Coef Variation & $15,070.04$ & $1,127.46$ & $1,937.41$ \\
Number missing & 0 & 0 & 0 \\
Median & -0.10 & 0.10 & 0.10 \\
Mode & -0.20 & 0.20 & 0.20 \\
& & Pne-sample t-test & 0.41 \\
& & & 0.6811 \\
\hline
\end{tabular}


Table A-67. Sample statistics for Sagittal MJS Right Initial, showing Edgewise sample alone $(n=31)$, MARA + Edgewise alone $(n=33)$ and the total sample $(n=$ 64).

\begin{tabular}{lccc}
\hline \multicolumn{1}{c}{ Statistic } & $\begin{array}{c}\text { Edgewise } \\
\text { Alone }\end{array}$ & $\begin{array}{c}\text { MARA+Edge } \\
\text { Alone }\end{array}$ & $\begin{array}{c}\text { Total } \\
\text { Sample }\end{array}$ \\
\hline Mean & 2.19 & 2.73 & 2.47 \\
Standard deviation & 0.43 & 0.67 & 0.62 \\
Standard error of mean & 0.08 & 0.12 & 0.08 \\
Upper 95\% Mean & 2.35 & 2.97 & 2.62 \\
Lower 95\% Mean & 2.03 & 2.49 & 2.31 \\
Sample size & 31 & 33 & 64 \\
Sample variance & 0.19 & 0.44 & 0.39 \\
Skewness (g1) & 0.49 & -0.04 & 0.48 \\
Kurtosis (g2) & 0.99 & -1.19 & -0.61 \\
Coef Variation & 19.89 & 24.37 & 25.31 \\
Number missing & 0 & 0 & 0 \\
Median & 2.1 & 2.7 & 2.4 \\
Mode & 2.4 & 2.6 & 1.8 \\
\hline
\end{tabular}


Table A-68. Sample statistics for Sagittal MJS Right Final, showing Edgewise sample alone $(n=31)$, MARA+Edgewise alone $(n=33)$ and the total sample $(n=$ 64).

\begin{tabular}{lccc}
\hline \multicolumn{1}{c}{ Statistic } & $\begin{array}{c}\text { Edgewise } \\
\text { Alone }\end{array}$ & $\begin{array}{c}\text { MARA+Edge } \\
\text { Alone }\end{array}$ & $\begin{array}{c}\text { Total } \\
\text { Sample }\end{array}$ \\
\hline Mean & 2.48 & 2.70 & 2.60 \\
Standard deviation & 0.65 & 0.86 & 0.77 \\
Standard error of mean & 0.12 & 0.15 & 0.10 \\
Upper 95\% Mean & 2.72 & 3.00 & 2.79 \\
Lower 95\% Mean & 2.25 & 2.40 & 2.40 \\
Sample size & 31 & 33 & 64 \\
Sample variance & 0.42 & 0.74 & 0.59 \\
Skewness (g1) & 0.89 & -0.20 & 0.23 \\
Kurtosis (g2) & 1.33 & 0.30 & 0.40 \\
Coef Variation & 26.20 & 31.79 & 29.54 \\
Number missing & 0 & 0 & 0 \\
Median & 2.4 & 2.8 & 2.5 \\
Mode & 2.1 & 2.4 & 2.4 \\
\hline
\end{tabular}


Table A-69. Sample statistics for Change in Sagittal MJS R, showing Edgewise sample alone $(n=31)$, MARA + Edgewise alone $(n=33)$ and the total sample $(n=$ 64).

\begin{tabular}{lccc}
\hline \multicolumn{1}{c}{ Statistic } & $\begin{array}{c}\text { Edgewise } \\
\text { Alone }\end{array}$ & $\begin{array}{c}\text { MARA+Edge } \\
\text { Alone }\end{array}$ & $\begin{array}{c}\text { Total } \\
\text { Sample }\end{array}$ \\
\hline Mean & 0.30 & -0.03 & 0.13 \\
Standard deviation & 0.60 & 0.80 & 0.72 \\
Standard error of mean & 0.11 & 0.14 & 0.09 \\
Upper 95\% Mean & 0.52 & 0.25 & 0.31 \\
Lower 95\% Mean & 0.08 & -0.31 & -0.05 \\
Sample size & 31 & 33 & 64 \\
Sample variance & 0.36 & 0.64 & 0.52 \\
Skewness (g1) & 0.55 & 1.05 & 0.66 \\
Kurtosis (g2) & 0.92 & 2.25 & 1.35 \\
Coef Variation & 203.20 & $-2,638.05$ & 565.47 \\
Number missing & 0 & 0 & 0 \\
Median & 0.30 & -0.10 & 0.10 \\
Mode & 0.10 & -0.80 & 0.10 \\
& & One-sample t-test & 1.41 \\
& & & 0.1621 \\
\hline
\end{tabular}


Table A-70. Sample statistics for Sagittal PJS Right Initial, showing Edgewise sample alone $(n=31)$, MARA+Edgewise alone $(n=33)$ and the total sample $(n=$ 64).

\begin{tabular}{lccc}
\hline \multicolumn{1}{c}{ Statistic } & $\begin{array}{c}\text { Edgewise } \\
\text { Alone }\end{array}$ & $\begin{array}{c}\text { MARA+Edge } \\
\text { Alone }\end{array}$ & $\begin{array}{c}\text { Total } \\
\text { Sample }\end{array}$ \\
\hline Mean & 2.84 & 2.74 & 2.79 \\
Standard deviation & 1.30 & 1.06 & 1.17 \\
Standard error of mean & 0.23 & 0.18 & 0.15 \\
Upper 95\% Mean & 3.32 & 3.11 & 3.08 \\
Lower 95\% Mean & 2.36 & 2.36 & 2.49 \\
Sample size & 31 & 33 & 64 \\
Sample variance & 1.70 & 1.12 & 1.38 \\
Skewness (g1) & 1.33 & 1.15 & 1.28 \\
Kurtosis (g2) & 0.82 & 1.93 & 1.23 \\
Coef Variation & 45.90 & 38.59 & 42.12 \\
Number missing & 0 & 0 & 0 \\
Median & 2.4 & 2.5 & 2.5 \\
Mode & 1.9 & 2.1 & 2.7 \\
\hline
\end{tabular}


Table A-71. Sample statistics for Sagittal PJS Right Final, showing Edgewise sample alone $(n=31)$, MARA + Edgewise alone $(n=33)$ and the total sample $(n=$ 64).

\begin{tabular}{lccc}
\hline \multicolumn{1}{c}{ Statistic } & $\begin{array}{c}\text { Edgewise } \\
\text { Alone }\end{array}$ & $\begin{array}{c}\text { MARA+Edge } \\
\text { Alone }\end{array}$ & $\begin{array}{c}\text { Total } \\
\text { Sample }\end{array}$ \\
\hline Mean & 2.93 & 2.42 & 2.67 \\
Standard deviation & 1.41 & 0.75 & 1.14 \\
Standard error of mean & 0.25 & 0.13 & 0.14 \\
Upper 95\% Mean & 3.45 & 2.69 & 2.95 \\
Lower 95\% Mean & 2.41 & 2.15 & 2.38 \\
Sample size & 31 & 33 & 64 \\
Sample variance & 2.00 & 0.57 & 1.31 \\
Skewness (g1) & 1.22 & 0.73 & 1.54 \\
Kurtosis (g2) & 0.85 & -0.03 & 2.61 \\
Coef Variation & 48.29 & 31.08 & 42.84 \\
Number missing & 0 & 0 & 0 \\
Median & 2.5 & 2.3 & 2.3 \\
Mode & 2.0 & 2.1 & 2.0 \\
\hline
\end{tabular}


Table A-72. Sample statistics for Change in Sagittal PJS R, showing Edgewise sample alone $(n=31)$, MARA + Edgewise alone $(n=33)$ and the total sample $(n=$ 64).

\begin{tabular}{lccc}
\hline \multicolumn{1}{c}{ Statistic } & $\begin{array}{c}\text { Edgewise } \\
\text { Alone }\end{array}$ & $\begin{array}{c}\text { MARA+Edge } \\
\text { Alone }\end{array}$ & $\begin{array}{c}\text { Total } \\
\text { Sample }\end{array}$ \\
\hline Mean & 0.09 & -0.32 & -0.12 \\
Standard deviation & 0.80 & 1.11 & 0.99 \\
Standard error of mean & 0.14 & 0.19 & 0.12 \\
Upper 95\% Mean & 0.39 & 0.08 & 0.13 \\
Lower 95\% Mean & -0.20 & -0.71 & -0.37 \\
Sample size & 31 & 33 & 64 \\
Sample variance & 0.65 & 1.23 & 0.98 \\
Skewness (g1) & 0.85 & -1.31 & -0.89 \\
Kurtosis (g2) & 0.68 & 6.85 & 6.34 \\
Coef Variation & 890.94 & -349.22 & -821.91 \\
Number missing & 0 & 0 & 0 \\
Median & -0.1 & -0.4 & -0.2 \\
Mode & -0.5 & -0.4 & -0.4 \\
& & One-sample t-test & 0.97 \\
& & & 0.3341 \\
\hline
\end{tabular}


Table A-73. Sample statistics for Sagittal FW Left Initial, showing Edgewise sample alone $(n=31)$, MARA + Edgewise alone $(n=33)$ and the total sample $(n=$ 64).

\begin{tabular}{lccc}
\hline \multicolumn{1}{c}{ Statistic } & $\begin{array}{c}\text { Edgewise } \\
\text { Alone }\end{array}$ & $\begin{array}{c}\text { MARA+Edge } \\
\text { Alone }\end{array}$ & $\begin{array}{c}\text { Total } \\
\text { Sample }\end{array}$ \\
\hline Mean & 17.48 & 17.20 & 17.33 \\
Standard deviation & 1.78 & 2.03 & 1.91 \\
Standard error of mean & 0.32 & 0.35 & 0.24 \\
Upper 95\% Mean & 18.13 & 17.92 & 17.81 \\
Lower 95\% Mean & 16.82 & 16.48 & 16.86 \\
Sample size & 31 & 33 & 64 \\
Sample variance & 3.17 & 4.14 & 3.63 \\
Skewness (g1) & 0.43 & 1.46 & 1.01 \\
Kurtosis (g2) & -0.62 & 3.55 & 1.68 \\
Coef Variation & 10.19 & 11.82 & 10.99 \\
Number missing & 0 & 0 & 0 \\
Median & 17.1 & 16.7 & 16.8 \\
Mode & 15.7 & 16.6 & 15.7 \\
\hline
\end{tabular}


Table A-74. Sample statistics for Sagittal FW Left Final, showing Edgewise sample alone $(n=31)$, MARA+Edgewise alone $(n=33)$ and the total sample $(n=64)$.

\begin{tabular}{lccc}
\hline \multicolumn{1}{c}{ Statistic } & $\begin{array}{c}\text { Edgewise } \\
\text { Alone }\end{array}$ & $\begin{array}{c}\text { MARA+Edge } \\
\text { Alone }\end{array}$ & $\begin{array}{c}\text { Total } \\
\text { Sample }\end{array}$ \\
\hline Mean & 17.61 & 17.55 & 17.58 \\
Standard deviation & 1.66 & 1.77 & 1.70 \\
Standard error of mean & 0.30 & 0.31 & 0.21 \\
Upper 95\% Mean & 18.22 & 18.18 & 18.01 \\
Lower 95\% Mean & 17.00 & 16.93 & 17.16 \\
Sample size & 31 & 33 & 64 \\
Sample variance & 2.75 & 3.14 & 2.90 \\
Skewness (g1) & 0.15 & 0.33 & 0.25 \\
Kurtosis (g2) & -0.39 & 0.11 & -0.18 \\
Coef Variation & 9.41 & 10.10 & 9.69 \\
Number missing & 0 & 0 & 0 \\
Median & 17.5 & 17.3 & 17.4 \\
Mode & 17.5 & 17.7 & 17.7 \\
\hline
\end{tabular}


Table A-75. Sample statistics for Change in Sagittal FW L, showing Edgewise sample alone $(n=31)$, MARA+Edgewise alone $(n=33)$ and the total sample $(n=$ 64).

\begin{tabular}{lccc}
\hline \multicolumn{1}{c}{ Statistic } & $\begin{array}{c}\text { Edgewise } \\
\text { Alone }\end{array}$ & $\begin{array}{c}\text { MARA+Edge } \\
\text { Alone }\end{array}$ & $\begin{array}{c}\text { Total } \\
\text { Sample }\end{array}$ \\
\hline Mean & 0.13 & 0.35 & 0.25 \\
Standard deviation & 1.28 & 1.45 & 1.36 \\
Standard error of mean & 0.23 & 0.25 & 0.17 \\
Upper 95\% Mean & 0.60 & 0.87 & 0.59 \\
Lower 95\% Mean & -0.34 & -0.16 & -0.09 \\
Sample size & 31 & 33 & 64 \\
Sample variance & 1.63 & 2.09 & 1.85 \\
Skewness (g1) & 0.70 & -2.51 & -1.18 \\
Kurtosis (g2) & 3.47 & 10.46 & 6.69 \\
Coef Variation & 965.20 & 407.70 & 550.92 \\
Number missing & 0 & 0 & 0 \\
Median & 0.2 & 0.5 & 0.4 \\
Mode & -0.2 & 1.1 & -0.2 \\
& & One-sample t-test & 1.45 \\
& & & 0.1514 \\
\hline
\end{tabular}


Table A-76. Sample statistics for Sagittal FD Left Initial, showing Edgewise sample alone $(n=31)$, MARA + Edgewise alone $(n=33)$ and the total sample $(n=$ 64).

\begin{tabular}{lccc}
\hline \multicolumn{1}{c}{ Statistic } & $\begin{array}{c}\text { Edgewise } \\
\text { Alone }\end{array}$ & $\begin{array}{c}\text { MARA+Edge } \\
\text { Alone }\end{array}$ & $\begin{array}{c}\text { Total } \\
\text { Sample }\end{array}$ \\
\hline Mean & 7.06 & 7.87 & 7.48 \\
Standard deviation & 1.01 & 1.15 & 1.15 \\
Standard error of mean & 0.18 & 0.20 & 0.14 \\
Upper 95\% Mean & 7.43 & 8.28 & 7.77 \\
Lower 95\% Mean & 6.69 & 7.46 & 7.19 \\
Sample size & 31 & 33 & 64 \\
Sample variance & 1.02 & 1.33 & 1.32 \\
Skewness (g1) & -0.88 & -0.24 & -0.27 \\
Kurtosis (g2) & 1.24 & -0.73 & 0.17 \\
Coef Variation & 14.27 & 14.63 & 15.38 \\
Number missing & 0 & 0 & 0 \\
Median & 7.2 & 7.9 & 7.5 \\
Mode & 7.2 & 8.3 & 7.2 \\
\hline
\end{tabular}


Table A-77. Sample statistics for Sagittal FD Left Final, showing Edgewise sample alone $(n=31)$, MARA+Edgewise alone $(n=33)$ and the total sample $(n=64)$.

\begin{tabular}{lccc}
\hline \multicolumn{1}{c}{ Statistic } & $\begin{array}{c}\text { Edgewise } \\
\text { Alone }\end{array}$ & $\begin{array}{c}\text { MARA+Edge } \\
\text { Alone }\end{array}$ & $\begin{array}{c}\text { Total } \\
\text { Sample }\end{array}$ \\
\hline Mean & 7.50 & 8.02 & 7.77 \\
Standard deviation & 1.11 & 1.12 & 1.13 \\
Standard error of mean & 0.20 & 0.19 & 0.14 \\
Upper 95\% Mean & 7.91 & 8.41 & 8.05 \\
Lower 95\% Mean & 7.09 & 7.62 & 7.48 \\
Sample size & 31 & 33 & 64 \\
Sample variance & 1.23 & 1.25 & 1.29 \\
Skewness (g1) & -0.98 & 0.25 & -0.30 \\
Kurtosis (g2) & 2.15 & -0.35 & 1.14 \\
Coef Variation & 14.80 & 13.93 & 14.61 \\
Number missing & 0 & 0 & 0 \\
Median & 7.3 & 7.9 & 7.8 \\
Mode & 8.5 & 6.8 & 8.5 \\
\hline
\end{tabular}


Table A-78. Sample statistics for Change in Sagittal FD L, showing Edgewise sample alone $(n=31)$, MARA+Edgewise alone $(n=33)$ and the total sample $(n=$ 64).

\begin{tabular}{lccc}
\hline \multicolumn{1}{c}{ Statistic } & $\begin{array}{c}\text { Edgewise } \\
\text { Alone }\end{array}$ & $\begin{array}{c}\text { MARA+Edge } \\
\text { Alone }\end{array}$ & $\begin{array}{c}\text { Total } \\
\text { Sample }\end{array}$ \\
\hline Mean & 0.44 & 0.15 & 0.29 \\
Standard deviation & 1.01 & 0.56 & 0.82 \\
Standard error of mean & 0.18 & 0.10 & 0.10 \\
Upper 95\% Mean & 0.81 & 0.35 & 0.49 \\
Lower 95\% Mean & 0.07 & -0.05 & 0.09 \\
Sample size & 31 & 33 & 64 \\
Sample variance & 1.02 & 0.32 & 0.67 \\
Skewness (g1) & 1.96 & -0.35 & 1.92 \\
Kurtosis (g2) & 7.27 & 1.48 & 9.51 \\
Coef Variation & 229.81 & 378.78 & 282.34 \\
Number missing & 0 & 0 & 0 \\
Median & 0.2 & 0.2 & 0.2 \\
Mode & 0.2 & 0.2 & 0.2 \\
& & P-value (two tail) & 0.0062 \\
\hline
\end{tabular}


Table A-79. Sample statistics for Sagittal FW Right Initial, showing Edgewise sample alone $(n=31)$, MARA+Edgewise alone $(n=33)$ and the total sample $(n=$ 64).

\begin{tabular}{lccc}
\hline \multicolumn{1}{c}{ Statistic } & $\begin{array}{c}\text { Edgewise } \\
\text { Alone }\end{array}$ & $\begin{array}{c}\text { MARA+Edge } \\
\text { Alone }\end{array}$ & $\begin{array}{c}\text { Total } \\
\text { Sample }\end{array}$ \\
\hline Mean & 18.07 & 16.73 & 17.38 \\
Standard deviation & 2.06 & 1.86 & 2.05 \\
Standard error of mean & 0.37 & 0.32 & 0.26 \\
Upper 95\% Mean & 18.82 & 17.39 & 17.89 \\
Lower 95\% Mean & 17.31 & 16.07 & 16.87 \\
Sample size & 31 & 33 & 64 \\
Sample variance & 4.24 & 3.45 & 4.22 \\
Skewness (g1) & 0.52 & 0.37 & 0.46 \\
Kurtosis (g2) & 0.21 & -0.47 & 0.05 \\
Coef Variation & 11.39 & 11.10 & 11.82 \\
Number missing & 0 & 0 & 0 \\
Median & 18.0 & 16.6 & 17.5 \\
Mode & 18.5 & 18.0 & 18.0 \\
\hline
\end{tabular}


Table A-80. Sample statistics for Sagittal FW Right Final, showing Edgewise sample alone $(n=31)$, MARA + Edgewise alone $(n=33)$ and the total sample $(n=$ 64).

\begin{tabular}{lccc}
\hline \multicolumn{1}{c}{ Statistic } & $\begin{array}{c}\text { Edgewise } \\
\text { Alone }\end{array}$ & $\begin{array}{c}\text { MARA+Edge } \\
\text { Alone }\end{array}$ & $\begin{array}{c}\text { Total } \\
\text { Sample }\end{array}$ \\
\hline Mean & 18.44 & 17.02 & 17.70 \\
Standard deviation & 1.91 & 1.68 & 1.92 \\
Standard error of mean & 0.34 & 0.29 & 0.24 \\
Upper 95\% Mean & 19.14 & 17.61 & 18.18 \\
Lower 95\% Mean & 17.73 & 16.42 & 17.22 \\
Sample size & 31 & 33 & 64 \\
Sample variance & 3.65 & 2.82 & 3.68 \\
Skewness (g1) & 0.08 & 0.13 & 0.21 \\
Kurtosis (g2) & -0.36 & -0.62 & -0.37 \\
Coef Variation & 10.36 & 9.88 & 10.84 \\
Number missing & 0 & 0 & 0 \\
Median & 18.5 & 16.6 & 17.7 \\
Mode & 19.1 & 16.0 & 16.5 \\
\hline
\end{tabular}


Table A-81. Sample statistics for Change in Sagittal FW R, showing Edgewise sample alone $(n=31)$, MARA + Edgewise alone $(n=33)$ and the total sample $(n=$ 64).

\begin{tabular}{lccc}
\hline \multicolumn{1}{c}{ Statistic } & $\begin{array}{c}\text { Edgewise } \\
\text { Alone }\end{array}$ & $\begin{array}{c}\text { MARA+Edge } \\
\text { Alone }\end{array}$ & $\begin{array}{c}\text { Total } \\
\text { Sample }\end{array}$ \\
\hline Mean & 0.37 & 0.28 & 0.32 \\
Standard deviation & 1.31 & 1.39 & 1.34 \\
Standard error of mean & 0.24 & 0.24 & 0.17 \\
Upper 95\% Mean & 0.85 & 0.78 & 0.66 \\
Lower 95\% Mean & -0.11 & -0.21 & -0.01 \\
Sample size & 31 & 33 & 64 \\
Sample variance & 1.71 & 1.93 & 1.80 \\
Skewness (g1) & -0.10 & -1.27 & -0.74 \\
Kurtosis (g2) & 0.54 & 3.93 & 2.32 \\
Coef Variation & 355.83 & 493.55 & 414.80 \\
Number missing & 0 & 0 & 0 \\
Median & 0.5 & 0.3 & 0.4 \\
Mode & -1.0 & 0.6 & 0.2 \\
& & P-value (two tail) & 0.0583 \\
\hline
\end{tabular}


Table A-82. Sample statistics for Sagittal FD Right Initial, showing Edgewise sample alone $(n=31)$, MARA + Edgewise alone $(n=33)$ and the total sample $(n=$ 64).

\begin{tabular}{lccc}
\hline \multicolumn{1}{c}{ Statistic } & $\begin{array}{c}\text { Edgewise } \\
\text { Alone }\end{array}$ & $\begin{array}{c}\text { MARA+Edge } \\
\text { Alone }\end{array}$ & $\begin{array}{c}\text { Total } \\
\text { Sample }\end{array}$ \\
\hline Mean & 7.37 & 7.85 & 7.62 \\
Standard deviation & 1.12 & 0.84 & 1.01 \\
Standard error of mean & 0.20 & 0.15 & 0.13 \\
Upper 95\% Mean & 7.78 & 8.14 & 7.87 \\
Lower 95\% Mean & 6.96 & 7.55 & 7.36 \\
Sample size & 31 & 33 & 64 \\
Sample variance & 1.26 & 0.71 & 1.02 \\
Skewness (g1) & 0.00 & 0.57 & -0.03 \\
Kurtosis (g2) & -0.67 & 0.29 & -0.08 \\
Coef Variation & 15.24 & 10.74 & 13.25 \\
Number missing & 0 & 0 & 0 \\
Median & 7.4 & 7.8 & 7.6 \\
Mode & 6.1 & 7.6 & 7.6 \\
\hline
\end{tabular}


Table A-83. Sample statistics for Sagittal FD Right Final, showing Edgewise sample alone $(n=31)$, MARA + Edgewise alone $(n=33)$ and the total sample $(n=$ 64).

\begin{tabular}{lccc}
\hline \multicolumn{1}{c}{ Statistic } & $\begin{array}{c}\text { Edgewise } \\
\text { Alone }\end{array}$ & $\begin{array}{c}\text { MARA+Edge } \\
\text { Alone }\end{array}$ & $\begin{array}{c}\text { Total } \\
\text { Sample }\end{array}$ \\
\hline Mean & 7.71 & 7.98 & 7.85 \\
Standard deviation & 1.02 & 1.07 & 1.05 \\
Standard error of mean & 0.18 & 0.19 & 0.13 \\
Upper 95\% Mean & 8.09 & 8.36 & 8.11 \\
Lower 95\% Mean & 7.33 & 7.61 & 7.59 \\
Sample size & 31 & 33 & 64 \\
Sample variance & 1.05 & 1.14 & 1.10 \\
Skewness (g1) & -0.01 & 0.03 & 0.03 \\
Kurtosis (g2) & -0.94 & 0.10 & -0.39 \\
Coef Variation & 13.28 & 13.37 & 13.34 \\
Number missing & 0 & 0 & 0 \\
Median & 7.8 & 8.0 & 7.9 \\
Mode & 6.2 & 7.3 & 7.8 \\
\hline
\end{tabular}


Table A-84. Sample statistics for Change in Sagittal FD R, showing Edgewise sample alone $(n=31)$, MARA+Edgewise alone $(n=33)$ and the total sample $(n=$ 64).

\begin{tabular}{lccc}
\hline \multicolumn{1}{c}{ Statistic } & $\begin{array}{c}\text { Edgewise } \\
\text { Alone }\end{array}$ & $\begin{array}{c}\text { MARA+Edge } \\
\text { Alone }\end{array}$ & $\begin{array}{c}\text { Total } \\
\text { Sample }\end{array}$ \\
\hline Mean & 0.34 & 0.14 & 0.24 \\
Standard deviation & 0.66 & 0.68 & 0.67 \\
Standard error of mean & 0.12 & 0.12 & 0.08 \\
Upper 95\% Mean & 0.58 & 0.38 & 0.40 \\
Lower 95\% Mean & 0.10 & -0.10 & 0.07 \\
Sample size & 31 & 33 & 64 \\
Sample variance & 0.44 & 0.46 & 0.45 \\
Skewness (g1) & -0.41 & -0.32 & -0.35 \\
Kurtosis (g2) & -0.76 & -0.16 & -0.49 \\
Coef Variation & 195.20 & 486.54 & 284.91 \\
Number missing & 0 & 0 & 0 \\
Median & 0.5 & 0.1 & 0.3 \\
Mode & 0.3 & 0.1 & 0.3 \\
& & P-value (two tail) & 0.0066 \\
\hline
\end{tabular}


Table A-85. Sample statistics for Sagittal CHW Left Initial, showing Edgewise sample alone $(n=31)$, MARA + Edgewise alone $(n=33)$ and the total sample $(n=$ 64).

\begin{tabular}{lccc}
\hline \multicolumn{1}{c}{ Statistic } & $\begin{array}{c}\text { Edgewise } \\
\text { Alone }\end{array}$ & $\begin{array}{c}\text { MARA+Edge } \\
\text { Alone }\end{array}$ & $\begin{array}{c}\text { Total } \\
\text { Sample }\end{array}$ \\
\hline Mean & 8.82 & 8.90 & 8.86 \\
Standard deviation & 1.25 & 1.26 & 1.24 \\
Standard error of mean & 0.22 & 0.22 & 0.16 \\
Upper 95\% Mean & 9.28 & 9.34 & 9.17 \\
Lower 95\% Mean & 8.36 & 8.45 & 8.55 \\
Sample size & 31 & 33 & 64 \\
Sample variance & 1.56 & 1.58 & 1.55 \\
Skewness (g1) & -0.66 & -0.23 & -0.42 \\
Kurtosis (g2) & 0.39 & -0.56 & -0.17 \\
Coef Variation & 14.15 & 14.12 & 14.03 \\
Number missing & 0 & 0 & 0 \\
Median & 8.9 & 8.9 & 8.9 \\
Mode & 8.9 & 8.9 & 8.9 \\
\hline
\end{tabular}


Table A-86. Sample statistics for Sagittal CHW Left Final, showing Edgewise sample alone $(n=31)$, MARA + Edgewise alone $(n=33)$ and the total sample $(n=$ 64).

\begin{tabular}{lccc}
\hline \multicolumn{1}{c}{ Statistic } & $\begin{array}{c}\text { Edgewise } \\
\text { Alone }\end{array}$ & $\begin{array}{c}\text { MARA+Edge } \\
\text { Alone }\end{array}$ & $\begin{array}{c}\text { Total } \\
\text { Sample }\end{array}$ \\
\hline Mean & 8.77 & 8.89 & 8.83 \\
Standard deviation & 1.50 & 1.14 & 1.32 \\
Standard error of mean & 0.27 & 0.20 & 0.17 \\
Upper 95\% Mean & 9.32 & 9.30 & 9.16 \\
Lower 95\% Mean & 8.22 & 8.49 & 8.50 \\
Sample size & 31 & 33 & 64 \\
Sample variance & 2.26 & 1.31 & 1.75 \\
Skewness (g1) & -0.59 & 0.18 & -0.38 \\
Kurtosis (g2) & -0.37 & 1.34 & 0.29 \\
Coef Variation & 17.14 & 12.87 & 14.95 \\
Number missing & 0 & 0 & 0 \\
Median & 8.9 & 8.9 & 8.9 \\
Mode & 8.6 & 9.3 & 9.3 \\
\hline
\end{tabular}


Table A-87. Sample statistics for Change in Sagittal CHW L, showing Edgewise sample alone $(n=31)$, MARA+Edgewise alone $(n=33)$ and the total sample $(n=$ 64).

\begin{tabular}{lccc}
\hline \multicolumn{1}{c}{ Statistic } & $\begin{array}{c}\text { Edgewise } \\
\text { Alone }\end{array}$ & $\begin{array}{c}\text { MARA+Edge } \\
\text { Alone }\end{array}$ & $\begin{array}{c}\text { Total } \\
\text { Sample }\end{array}$ \\
\hline Mean & -0.05 & 0.00 & -0.03 \\
Standard deviation & 0.85 & 0.86 & 0.85 \\
Standard error of mean & 0.15 & 0.15 & 0.11 \\
Upper 95\% Mean & 0.26 & 0.30 & 0.19 \\
Lower 95\% Mean & -0.36 & -0.31 & -0.24 \\
Sample size & 31 & 33 & 64 \\
Sample variance & 0.72 & 0.75 & 0.72 \\
Skewness (g1) & -0.68 & 0.65 & 0.03 \\
Kurtosis (g2) & 0.95 & 1.55 & 1.15 \\
Coef Variation & $-1,751.90$ & $-28,536.95$ & $-3,400.09$ \\
Number missing & 0 & 0 & 0 \\
Median & 0.0 & -0.1 & 0.0 \\
Mode & 0.0 & 0.1 & 0.1 \\
& & One-sample t-test & -0.24 \\
& & & 0.8147 \\
\hline
\end{tabular}


Table A-88. Sample statistics for Sagittal CHH Left Initial, showing Edgewise sample alone $(n=31)$, MARA + Edgewise alone $(n=33)$ and the total sample $(n=$ 64).

\begin{tabular}{lccc}
\hline \multicolumn{1}{c}{ Statistic } & $\begin{array}{c}\text { Edgewise } \\
\text { Alone }\end{array}$ & $\begin{array}{c}\text { MARA+Edge } \\
\text { Alone }\end{array}$ & $\begin{array}{c}\text { Total } \\
\text { Sample }\end{array}$ \\
\hline Mean & 6.56 & 6.43 & 6.49 \\
Standard deviation & 1.02 & 0.99 & 1.00 \\
Standard error of mean & 0.18 & 0.17 & 0.12 \\
Upper 95\% Mean & 6.94 & 6.78 & 6.74 \\
Lower 95\% Mean & 6.19 & 6.07 & 6.24 \\
Sample size & 31 & 33 & 64 \\
Sample variance & 1.04 & 0.99 & 1.00 \\
Skewness (g1) & 0.99 & 0.68 & 0.81 \\
Kurtosis (g2) & 0.46 & 0.53 & 0.41 \\
Coef Variation & 15.50 & 15.46 & 15.39 \\
Number missing & 0 & 0 & 0 \\
Median & 6.2 & 6.3 & 6.3 \\
Mode & 6.2 & 6.1 & 6.2 \\
\hline
\end{tabular}


Table A-89. Sample statistics for Sagittal CHH Left Final, showing Edgewise sample alone $(n=31)$, MARA + Edgewise alone $(n=33)$ and the total sample $(n=$ 64).

\begin{tabular}{lccc}
\hline \multicolumn{1}{c}{ Statistic } & $\begin{array}{c}\text { Edgewise } \\
\text { Alone }\end{array}$ & $\begin{array}{c}\text { MARA+Edge } \\
\text { Alone }\end{array}$ & $\begin{array}{c}\text { Total } \\
\text { Sample }\end{array}$ \\
\hline Mean & 6.61 & 6.59 & 6.60 \\
Standard deviation & 0.92 & 1.02 & 0.97 \\
Standard error of mean & 0.17 & 0.18 & 0.12 \\
Upper 95\% Mean & 6.95 & 6.95 & 6.84 \\
Lower 95\% Mean & 6.28 & 6.23 & 6.36 \\
Sample size & 31 & 33 & 64 \\
Sample variance & 0.85 & 1.04 & 0.93 \\
Skewness (g1) & 0.00 & 0.43 & 0.25 \\
Kurtosis (g2) & -1.28 & 0.39 & -0.31 \\
Coef Variation & 13.92 & 15.47 & 14.62 \\
Number missing & 0 & 0 & 0 \\
Median & 6.5 & 6.5 & 6.5 \\
Mode & 7.5 & 6.0 & 6.0 \\
\hline
\end{tabular}


Table A-90. Sample statistics for Change in Sagittal CHH L, showing Edgewise sample alone $(n=31)$, MARA+Edgewise alone $(n=33)$ and the total sample $(n=$ 64).

\begin{tabular}{lccc}
\hline \multicolumn{1}{c}{ Statistic } & $\begin{array}{c}\text { Edgewise } \\
\text { Alone }\end{array}$ & $\begin{array}{c}\text { MARA+Edge } \\
\text { Alone }\end{array}$ & $\begin{array}{c}\text { Total } \\
\text { Sample }\end{array}$ \\
\hline Mean & 0.05 & 0.16 & 0.11 \\
Standard deviation & 0.90 & 0.72 & 0.81 \\
Standard error of mean & 0.16 & 0.13 & 0.10 \\
Upper 95\% Mean & 0.38 & 0.42 & 0.31 \\
Lower 95\% Mean & -0.28 & -0.09 & -0.10 \\
Sample size & 31 & 33 & 64 \\
Sample variance & 0.81 & 0.52 & 0.65 \\
Skewness (g1) & -0.54 & 0.36 & -0.26 \\
Kurtosis (g2) & 1.08 & -0.58 & 0.74 \\
Coef Variation & $1,864.49$ & 447.35 & 760.54 \\
Number missing & 0 & 0 & 0 \\
Median & 0.2 & 0.1 & 0.1 \\
Mode & -0.4 & -0.5 & 0.4 \\
& & One-sample t-test & 1.05 \\
& & & 0.2969 \\
\hline
\end{tabular}


Table A-91. Sample statistics for Sagittal CH Left Initial, showing Edgewise sample alone $(n=31)$, MARA+Edgewise alone $(n=33)$ and the total sample $(n=$ 64).

\begin{tabular}{lccc}
\hline \multicolumn{1}{c}{ Statistic } & $\begin{array}{c}\text { Edgewise } \\
\text { Alone }\end{array}$ & $\begin{array}{c}\text { MARA+Edge } \\
\text { Alone }\end{array}$ & $\begin{array}{c}\text { Total } \\
\text { Sample }\end{array}$ \\
\hline Mean & 16.40 & 14.42 & 15.38 \\
Standard deviation & 2.47 & 2.24 & 2.54 \\
Standard error of mean & 0.44 & 0.39 & 0.32 \\
Upper 95\% Mean & 17.31 & 15.21 & 16.01 \\
Lower 95\% Mean & 15.49 & 13.62 & 14.74 \\
Sample size & 31 & 33 & 64 \\
Sample variance & 6.12 & 5.04 & 6.47 \\
Skewness (g1) & 0.04 & 0.64 & 0.34 \\
Kurtosis (g2) & 0.17 & -0.44 & -0.44 \\
Coef Variation & 15.09 & 15.57 & 16.54 \\
Number missing & 0 & 0 & 0 \\
Median & 16.4 & 13.7 & 15.3 \\
Mode & 16.5 & 13.0 & 13.1 \\
\hline
\end{tabular}


Table A-92. Sample statistics for Sagittal CH Left Final, showing Edgewise sample alone $(n=31)$, MARA+Edgewise alone $(n=33)$ and the total sample $(n=64)$.

\begin{tabular}{lccc}
\hline \multicolumn{1}{c}{ Statistic } & $\begin{array}{c}\text { Edgewise } \\
\text { Alone }\end{array}$ & $\begin{array}{c}\text { MARA+Edge } \\
\text { Alone }\end{array}$ & $\begin{array}{c}\text { Total } \\
\text { Sample }\end{array}$ \\
\hline Mean & 16.44 & 15.81 & 16.11 \\
Standard deviation & 3.05 & 2.49 & 2.77 \\
Standard error of mean & 0.55 & 0.43 & 0.35 \\
Upper 95\% Mean & 17.55 & 16.69 & 16.81 \\
Lower 95\% Mean & 15.32 & 14.93 & 15.42 \\
Sample size & 31 & 33 & 64 \\
Sample variance & 9.29 & 6.18 & 7.66 \\
Skewness (g1) & 0.68 & 0.65 & 0.71 \\
Kurtosis (g2) & 0.09 & 0.11 & 0.21 \\
Coef Variation & 18.54 & 15.72 & 17.18 \\
Number missing & 0 & 0 & 0 \\
Median & 15.9 & 15.3 & 15.5 \\
Mode & 14.8 & 17.9 & 15.4 \\
\hline
\end{tabular}


Table A-93. Sample statistics for Change in Sagittal CH L, showing Edgewise sample alone $(n=31)$, MARA + Edgewise alone $(n=33)$ and the total sample $(n=$ 64).

\begin{tabular}{lccc}
\hline \multicolumn{1}{c}{ Statistic } & $\begin{array}{c}\text { Edgewise } \\
\text { Alone }\end{array}$ & $\begin{array}{c}\text { MARA+Edge } \\
\text { Alone }\end{array}$ & $\begin{array}{c}\text { Total } \\
\text { Sample }\end{array}$ \\
\hline Mean & 0.04 & 1.39 & 0.74 \\
Standard deviation & 1.98 & 1.48 & 1.85 \\
Standard error of mean & 0.36 & 0.26 & 0.23 \\
Upper 95\% Mean & 0.76 & 1.92 & 1.20 \\
Lower 95\% Mean & -0.69 & 0.87 & 0.27 \\
Sample size & 31 & 33 & 64 \\
Sample variance & 3.91 & 2.18 & 3.44 \\
Skewness (g1) & -0.61 & 0.08 & -0.60 \\
Kurtosis (g2) & -0.22 & -0.94 & 0.40 \\
Coef Variation & $5,573.80$ & 105.86 & 251.91 \\
Number missing & 0 & 0 & 0 \\
Median & 0.2 & 1.4 & 0.8 \\
Mode & 0.2 & 0.0 & 0.0 \\
& & One-sample t-test & 3.18 \\
& & & 0.0023 \\
\hline
\end{tabular}


Table A-94. Sample statistics for Sagittal CHW Right Initial, showing Edgewise sample alone $(n=31)$, MARA+Edgewise alone $(n=33)$ and the total sample $(n=$ 64).

\begin{tabular}{lccc}
\hline \multicolumn{1}{c}{ Statistic } & $\begin{array}{c}\text { Edgewise } \\
\text { Alone }\end{array}$ & $\begin{array}{c}\text { MARA+Edge } \\
\text { Alone }\end{array}$ & $\begin{array}{c}\text { Total } \\
\text { Sample }\end{array}$ \\
\hline Mean & 9.36 & 8.87 & 9.11 \\
Standard deviation & 0.99 & 1.35 & 1.21 \\
Standard error of mean & 0.18 & 0.24 & 0.15 \\
Upper 95\% Mean & 9.73 & 9.35 & 9.41 \\
Lower 95\% Mean & 9.00 & 8.39 & 8.81 \\
Sample size & 31 & 33 & 64 \\
Sample variance & 0.99 & 1.84 & 1.46 \\
Skewness (g1) & -0.50 & 0.17 & -0.19 \\
Kurtosis (g2) & -0.03 & -0.59 & -0.48 \\
Coef Variation & 10.61 & 15.27 & 13.28 \\
Number missing & 0 & 0 & 0 \\
Median & 9.4 & 8.7 & 9.3 \\
Mode & 10.6 & 8.0 & 8.0 \\
\hline
\end{tabular}


Table A-95. Sample statistics for Sagittal CHW Right Final, showing Edgewise sample alone $(n=31)$, MARA + Edgewise alone $(n=33)$ and the total sample $(n=$ 64).

\begin{tabular}{lccc}
\hline \multicolumn{1}{c}{ Statistic } & $\begin{array}{c}\text { Edgewise } \\
\text { Alone }\end{array}$ & $\begin{array}{c}\text { MARA+Edge } \\
\text { Alone }\end{array}$ & $\begin{array}{c}\text { Total } \\
\text { Sample }\end{array}$ \\
\hline Mean & 9.35 & 8.72 & 9.02 \\
Standard deviation & 1.11 & 1.37 & 1.28 \\
Standard error of mean & 0.20 & 0.24 & 0.16 \\
Upper 95\% Mean & 9.76 & 9.20 & 9.34 \\
Lower 95\% Mean & 8.94 & 8.23 & 8.70 \\
Sample size & 31 & 33 & 64 \\
Sample variance & 1.24 & 1.88 & 1.65 \\
Skewness (g1) & -0.22 & 0.67 & 0.19 \\
Kurtosis (g2) & -0.60 & 0.25 & -0.45 \\
Coef Variation & 11.92 & 15.74 & 14.24 \\
Number missing & 0 & & 0 \\
Median & 9.5 & 8.8 & 8.9 \\
Mode & 8.9 & 7.2 & 8.9 \\
\hline
\end{tabular}


Table A-96. Sample statistics for Change in Sagittal CHW R, showing Edgewise sample alone $(n=31)$, MARA+Edgewise alone $(n=33)$ and the total sample $(n=$ 64).

\begin{tabular}{lccc}
\hline \multicolumn{1}{c}{ Statistic } & $\begin{array}{c}\text { Edgewise } \\
\text { Alone }\end{array}$ & $\begin{array}{c}\text { MARA+Edge } \\
\text { Alone }\end{array}$ & $\begin{array}{c}\text { Total } \\
\text { Sample }\end{array}$ \\
\hline Mean & & & \\
Standard deviation & -0.01 & -0.16 & -0.09 \\
Standard error of mean & 0.80 & 0.87 & 0.83 \\
Upper 95\% Mean & 0.14 & 0.15 & 0.10 \\
Lower 95\% Mean & 0.28 & 0.15 & 0.12 \\
Sample size & -0.31 & -0.47 & -0.30 \\
Sample variance & 31 & 33 & 64 \\
Skewness (g1) & 0.65 & 0.75 & 0.70 \\
Kurtosis (g2) & -0.47 & -0.25 & -0.35 \\
Coef Variation & 0.34 & 0.39 & 0.24 \\
Number missing & $-6,234.59$ & -551.20 & -953.90 \\
Median & 0 & 0 & 0 \\
Mode & 0.1 & -0.2 & -0.1 \\
& -0.1 & -0.7 & -0.4 \\
& & One-sample t-test & -0.84 \\
& & & 0.4048 \\
\hline
\end{tabular}


Table A-97. Sample statistics for Sagittal CHH Right Initial, showing Edgewise sample alone $(n=31)$, MARA+Edgewise alone $(n=33)$ and the total sample $(n=$ 64).

\begin{tabular}{lccc}
\hline \multicolumn{1}{c}{ Statistic } & $\begin{array}{c}\text { Edgewise } \\
\text { Alone }\end{array}$ & $\begin{array}{c}\text { MARA+Edge } \\
\text { Alone }\end{array}$ & $\begin{array}{c}\text { Total } \\
\text { Sample }\end{array}$ \\
\hline Mean & 6.69 & 6.33 & 6.51 \\
Standard deviation & 1.00 & 0.79 & 0.91 \\
Standard error of mean & 0.18 & 0.14 & 0.11 \\
Upper 95\% Mean & 7.06 & 6.61 & 6.74 \\
Lower 95\% Mean & 6.33 & 6.05 & 6.28 \\
Sample size & 31 & 33 & 64 \\
Sample variance & 1.01 & 0.63 & 0.83 \\
Skewness (g1) & -0.18 & -0.21 & -0.04 \\
Kurtosis (g2) & -0.51 & 0.87 & -0.07 \\
Coef Variation & 15.00 & 12.51 & 14.01 \\
Number missing & 0 & 0 & 0 \\
Median & 6.5 & 6.3 & 6.4 \\
Mode & 6.4 & 5.8 & 6.4 \\
\hline
\end{tabular}


Table A-98. Sample statistics for Sagittal CHH Right Final, showing Edgewise sample alone $(n=31)$, MARA+Edgewise alone $(n=33)$ and the total sample $(n=$ 64).

\begin{tabular}{lccc}
\hline \multicolumn{1}{c}{ Statistic } & $\begin{array}{c}\text { Edgewise } \\
\text { Alone }\end{array}$ & $\begin{array}{c}\text { MARA+Edge } \\
\text { Alone }\end{array}$ & $\begin{array}{c}\text { Total } \\
\text { Sample }\end{array}$ \\
\hline Mean & 7.15 & 6.52 & 6.82 \\
Standard deviation & 1.22 & 0.95 & 1.12 \\
Standard error of mean & 0.22 & 0.16 & 0.14 \\
Upper 95\% Mean & 7.59 & 6.85 & 7.10 \\
Lower 95\% Mean & 6.70 & 6.18 & 6.54 \\
Sample size & 31 & 33 & 64 \\
Sample variance & 1.49 & 0.89 & 1.26 \\
Skewness (g1) & 0.87 & 0.31 & 0.80 \\
Kurtosis (g2) & -0.09 & -0.27 & 0.49 \\
Coef Variation & 17.06 & 14.51 & 16.46 \\
Number missing & 0 & 0 & 0 \\
Median & 6.5 & 6.4 & 6.5 \\
Mode & 6.2 & 6.1 & 6.2 \\
\hline
\end{tabular}


Table A-99. Sample statistics for Change in Sagittal CHH R, showing Edgewise sample alone $(n=31)$, MARA+Edgewise alone $(n=33)$ and the total sample $(n=$ 64).

\begin{tabular}{lccc}
\hline \multicolumn{1}{c}{ Statistic } & $\begin{array}{c}\text { Edgewise } \\
\text { Alone }\end{array}$ & $\begin{array}{c}\text { MARA+Edge } \\
\text { Alone }\end{array}$ & $\begin{array}{c}\text { Total } \\
\text { Sample }\end{array}$ \\
\hline Mean & 0.45 & 0.18 & 0.31 \\
Standard deviation & 1.06 & 0.91 & 0.99 \\
Standard error of mean & 0.19 & 0.16 & 0.12 \\
Upper 95\% Mean & 0.84 & 0.51 & 0.56 \\
Lower 95\% Mean & 0.06 & -0.14 & 0.07 \\
Sample size & 31 & 33 & 64 \\
Sample variance & 1.13 & 0.82 & 0.97 \\
Skewness (g1) & 0.58 & -0.18 & 0.33 \\
Kurtosis (g2) & 1.06 & -0.30 & 0.74 \\
Coef Variation & 235.65 & 489.72 & 314.18 \\
Number missing & 0 & 0 & 0 \\
Median & 0.4 & 0.2 & 0.3 \\
Mode & 0.0 & 0.6 & 0.6 \\
& & One-sample t-test & 2.55 \\
& & & 0.0133 \\
\hline
\end{tabular}


Table A-100. Sample statistics for Sagittal CH Right Initial, showing Edgewise sample alone $(n=31)$, MARA+Edgewise alone $(n=33)$ and the total sample $(n=$ 64).

\begin{tabular}{lccc}
\hline \multicolumn{1}{c}{ Statistic } & $\begin{array}{c}\text { Edgewise } \\
\text { Alone }\end{array}$ & $\begin{array}{c}\text { MARA+Edge } \\
\text { Alone }\end{array}$ & $\begin{array}{c}\text { Total } \\
\text { Sample }\end{array}$ \\
\hline Mean & 16.35 & 14.22 & 15.25 \\
Standard deviation & 2.42 & 2.57 & 2.70 \\
Standard error of mean & 0.43 & 0.45 & 0.34 \\
Upper 95\% Mean & 17.23 & 15.14 & 15.93 \\
Lower 95\% Mean & 15.46 & 13.31 & 14.58 \\
Sample size & 31 & 33 & 64 \\
Sample variance & 5.84 & 6.60 & 7.28 \\
Skewness (g1) & -0.24 & 0.88 & 0.23 \\
Kurtosis (g2) & 0.19 & 0.10 & -0.74 \\
Coef Variation & 14.79 & 18.06 & 17.69 \\
Number missing & 0 & 0 & 0 \\
Median & 16.3 & 13.3 & 15.1 \\
Mode & 15.9 & 12.1 & 16.8 \\
\hline
\end{tabular}


Table A-101. Sample statistics for Sagittal CH Right Final, showing Edgewise sample alone $(n=31)$, MARA+Edgewise alone $(n=33)$ and the total sample $(n=$ 64).

\begin{tabular}{lccc}
\hline \multicolumn{1}{c}{ Statistic } & $\begin{array}{c}\text { Edgewise } \\
\text { Alone }\end{array}$ & $\begin{array}{c}\text { MARA+Edge } \\
\text { Alone }\end{array}$ & $\begin{array}{c}\text { Total } \\
\text { Sample }\end{array}$ \\
\hline Mean & 16.56 & 15.73 & 16.13 \\
Standard deviation & 2.84 & 2.54 & 2.70 \\
Standard error of mean & 0.51 & 0.44 & 0.34 \\
Upper 95\% Mean & 17.61 & 16.63 & 16.81 \\
Lower 95\% Mean & 15.52 & 14.83 & 15.46 \\
Sample size & 31 & 33 & 64 \\
Sample variance & 8.09 & 6.46 & 7.31 \\
Skewness (g1) & 0.14 & 0.37 & 0.28 \\
Kurtosis (g2) & -0.25 & -0.24 & -0.32 \\
Coef Variation & 17.17 & 16.16 & 16.76 \\
Number missing & 0 & 0 & 0 \\
Median & 16.5 & 15.5 & 15.8 \\
Mode & 14.3 & 14.1 & 15.5 \\
\hline
\end{tabular}


Table A-102. Sample statistics for Change in Sagittal CH Right, showing Edgewise sample alone $(n=31)$, MARA+Edgewise alone $(n=33)$ and the total sample $(n=64)$.

\begin{tabular}{lccc}
\hline \multicolumn{1}{c}{ Statistic } & $\begin{array}{c}\text { Edgewise } \\
\text { Alone }\end{array}$ & $\begin{array}{c}\text { MARA+Edge } \\
\text { Alone }\end{array}$ & $\begin{array}{c}\text { Total } \\
\text { Sample }\end{array}$ \\
\hline Mean & 0.22 & 1.50 & 0.88 \\
Standard deviation & 1.55 & 1.51 & 1.65 \\
Standard error of mean & 0.28 & 0.26 & 0.21 \\
Upper 95\% Mean & 0.79 & 2.04 & 1.29 \\
Lower 95\% Mean & -0.35 & 0.97 & 0.47 \\
Sample size & 31 & 33 & 64 \\
Sample variance & 2.39 & 2.28 & 2.71 \\
Skewness (g1) & -0.76 & -0.84 & -0.63 \\
Kurtosis (g2) & 0.69 & 0.46 & 0.27 \\
Coef Variation & 704.52 & 100.43 & 186.90 \\
Number missing & 0 & 0 & 0 \\
Median & 0.60 & 1.80 & 1.05 \\
Mode & 0.90 & 1.40 & 0.90 \\
& & One-sample t-test & 4.28 \\
& & & 0.0001 \\
\hline
\end{tabular}


Table A-103. Sample statistics for Sagittal EAM-AE Left Initial, showing Edgewise sample alone $(n=31)$, MARA+Edgewise alone $(n=33)$ and the total sample $(n=64)$.

\begin{tabular}{lccc}
\hline \multicolumn{1}{c}{ Statistic } & $\begin{array}{c}\text { Edgewise } \\
\text { Alone }\end{array}$ & $\begin{array}{c}\text { MARA+Edge } \\
\text { Alone }\end{array}$ & $\begin{array}{c}\text { Total } \\
\text { Sample }\end{array}$ \\
\hline Mean & 23.06 & 22.85 & 22.95 \\
Standard deviation & 1.99 & 1.97 & 1.97 \\
Standard error of mean & 0.36 & 0.34 & 0.25 \\
Upper 95\% Mean & 23.79 & 23.55 & 23.44 \\
Lower 95\% Mean & 22.33 & 22.15 & 22.46 \\
Sample size & 31 & 33 & 64 \\
Sample variance & 3.96 & 3.89 & 3.88 \\
Skewness (g1) & -0.34 & -0.15 & -0.24 \\
Kurtosis (g2) & -1.10 & -0.32 & -0.76 \\
Coef Variation & 8.63 & 8.63 & 8.58 \\
Number missing & 0 & 0 & 0 \\
Median & 23.5 & 23.0 & 23.1 \\
Mode & 19.8 & 19.5 & 23.0 \\
\hline
\end{tabular}


Table A-104. Sample statistics for Sagittal EAM-AE Left Final, showing Edgewise sample alone $(n=31)$, MARA + Edgewise alone $(n=33)$ and the total sample $(n=$ 64).

\begin{tabular}{lccc}
\hline \multicolumn{1}{c}{ Statistic } & $\begin{array}{c}\text { Edgewise } \\
\text { Alone }\end{array}$ & $\begin{array}{c}\text { MARA+Edge } \\
\text { Alone }\end{array}$ & $\begin{array}{c}\text { Total } \\
\text { Sample }\end{array}$ \\
\hline Mean & 23.96 & 23.78 & 23.87 \\
Standard deviation & 1.88 & 2.25 & 2.06 \\
Standard error of mean & 0.34 & 0.39 & 0.26 \\
Upper 95\% Mean & 24.66 & 24.58 & 24.39 \\
Lower 95\% Mean & 23.27 & 22.99 & 23.36 \\
Sample size & 31 & 33 & 64 \\
Sample variance & 3.55 & 5.05 & 4.26 \\
Skewness (g1) & -0.67 & 0.03 & -0.24 \\
Kurtosis (g2) & 0.01 & -0.18 & -0.17 \\
Coef Variation & 7.86 & 9.45 & 8.65 \\
Number missing & 0 & 0 & 0 \\
Median & 24.1 & 24.2 & 24.2 \\
Mode & 24.1 & 22.6 & 24.5 \\
\hline
\end{tabular}


Table A-105. Sample statistics for Change in Sagittal EAM-AE L, showing Edgewise sample alone $(n=31)$, MARA+Edgewise alone $(n=33)$ and the total sample $(n=64)$.

\begin{tabular}{lccc}
\hline \multicolumn{1}{c}{ Statistic } & $\begin{array}{c}\text { Edgewise } \\
\text { Alone }\end{array}$ & $\begin{array}{c}\text { MARA+Edge } \\
\text { Alone }\end{array}$ & $\begin{array}{c}\text { Total } \\
\text { Sample }\end{array}$ \\
\hline Mean & 0.90 & 0.94 & 0.92 \\
Standard deviation & 1.18 & 0.99 & 1.08 \\
Standard error of mean & 0.21 & 0.17 & 0.13 \\
Upper 95\% Mean & 1.33 & 1.29 & 1.19 \\
Lower 95\% Mean & 0.47 & 0.58 & 0.65 \\
Sample size & 31 & 33 & 64 \\
Sample variance & 1.39 & 0.99 & 1.17 \\
Skewness (g1) & 1.40 & 0.67 & 1.09 \\
Kurtosis (g2) & 5.10 & 0.60 & 3.27 \\
Coef Variation & 131.16 & 106.12 & 117.50 \\
Number missing & 0 & 0 & 0 \\
Median & 0.7 & 0.8 & 0.7 \\
Mode & 0.5 & 1.3 & 1.3 \\
& & One-sample t-test & 6.81 \\
& & & $<0.0001$ \\
\hline
\end{tabular}


Table A-106. Sample statistics for Sagittal EAM-C Left Initial, showing Edgewise sample alone $(n=31)$, MARA+Edgewise alone $(n=33)$ and the total sample $(n=$ 64).

\begin{tabular}{lccc}
\hline \multicolumn{1}{c}{ Statistic } & $\begin{array}{c}\text { Edgewise } \\
\text { Alone }\end{array}$ & $\begin{array}{c}\text { MARA+Edge } \\
\text { Alone }\end{array}$ & $\begin{array}{c}\text { Total } \\
\text { Sample }\end{array}$ \\
\hline Mean & 12.74 & 13.22 & 12.99 \\
Standard deviation & 1.45 & 1.48 & 1.47 \\
Standard error of mean & 0.26 & 0.26 & 0.18 \\
Upper 95\% Mean & 13.27 & 13.75 & 13.36 \\
Lower 95\% Mean & 12.21 & 12.70 & 12.62 \\
Sample size & 31 & 33 & 64 \\
Sample variance & 2.11 & 2.19 & 2.18 \\
Skewness (g1) & 0.07 & 0.31 & 0.20 \\
Kurtosis (g2) & -0.39 & -0.33 & -0.32 \\
Coef Variation & 11.40 & 11.19 & 11.35 \\
Number missing & 0 & 0 & 0 \\
Median & 12.7 & 13.2 & 13.1 \\
Mode & 11.6 & 11.3 & 12.0 \\
\hline
\end{tabular}


Table A-107. Sample statistics for Sagittal EAM-C Left Final, showing Edgewise sample alone $(n=31)$, MARA+Edgewise alone $(n=33)$ and the total sample $(n=$ 64).

\begin{tabular}{lccc}
\hline \multicolumn{1}{c}{ Statistic } & $\begin{array}{c}\text { Edgewise } \\
\text { Alone }\end{array}$ & $\begin{array}{c}\text { MARA+Edge } \\
\text { Alone }\end{array}$ & $\begin{array}{c}\text { Total } \\
\text { Sample }\end{array}$ \\
\hline Mean & 13.73 & 13.38 & 13.55 \\
Standard deviation & 2.08 & 1.50 & 1.80 \\
Standard error of mean & 0.37 & 0.26 & 0.22 \\
Upper 95\% Mean & 14.49 & 13.92 & 14.00 \\
Lower 95\% Mean & 12.96 & 12.85 & 13.10 \\
Sample size & 31 & 33 & 64 \\
Sample variance & 4.31 & 2.26 & 3.23 \\
Skewness (g1) & 1.23 & -0.09 & 0.94 \\
Kurtosis (g2) & 4.42 & -0.61 & 3.82 \\
Coef Variation & 15.12 & 11.22 & 13.26 \\
Number missing & 0 & 0 & 0 \\
Median & 13.5 & 13.5 & 13.5 \\
Mode & 13.3 & 11.9 & 13.5 \\
\hline
\end{tabular}


Table A-108. Sample statistics for Change in Sagittal EAM-C L, showing Edgewise sample alone $(n=31)$, MARA+Edgewise alone $(n=33)$ and the total sample $(n=64)$.

\begin{tabular}{lccc}
\hline \multicolumn{1}{c}{ Statistic } & $\begin{array}{c}\text { Edgewise } \\
\text { Alone }\end{array}$ & $\begin{array}{c}\text { MARA+Edge } \\
\text { Alone }\end{array}$ & $\begin{array}{c}\text { Total } \\
\text { Sample }\end{array}$ \\
\hline Mean & 0.98 & 0.16 & 0.56 \\
Standard deviation & 1.84 & 1.02 & 1.52 \\
Standard error of mean & 0.33 & 0.18 & 0.19 \\
Upper 95\% Mean & 1.66 & 0.53 & 0.94 \\
Lower 95\% Mean & 0.31 & -0.20 & 0.18 \\
Sample size & 31 & 33 & 64 \\
Sample variance & 3.37 & 1.05 & 2.31 \\
Skewness (g1) & 4.13 & 0.42 & 3.83 \\
Kurtosis (g2) & 20.50 & 0.97 & 23.43 \\
Coef Variation & 186.59 & 625.05 & 270.77 \\
Number missing & 0 & 0 & 0 \\
Median & 0.7 & 0.1 & 0.4 \\
Mode & 0.6 & -0.6 & 0.6 \\
& & One-sample t-test & 2.95 \\
& & & 0.0044 \\
\hline
\end{tabular}


Table A-109. Sample statistics for Sagittal EAM-P Left Initial, showing Edgewise sample alone $(n=31)$, MARA+Edgewise alone $(n=33)$ and the total sample $(n=$ 64).

\begin{tabular}{lccc}
\hline \multicolumn{1}{c}{ Statistic } & $\begin{array}{c}\text { Edgewise } \\
\text { Alone }\end{array}$ & $\begin{array}{c}\text { MARA+Edge } \\
\text { Alone }\end{array}$ & $\begin{array}{c}\text { Total } \\
\text { Sample }\end{array}$ \\
\hline Mean & 5.45 & 5.82 & 5.64 \\
Standard deviation & 1.13 & 0.97 & 1.06 \\
Standard error of mean & 0.20 & 0.17 & 0.13 \\
Upper 95\% Mean & 5.86 & 6.17 & 5.91 \\
Lower 95\% Mean & 5.03 & 5.48 & 5.38 \\
Sample size & 31 & 33 & 64 \\
Sample variance & 1.27 & 0.95 & 1.12 \\
Skewness (g1) & 0.14 & -0.07 & -0.03 \\
Kurtosis (g2) & -0.67 & -0.48 & -0.63 \\
Coef Variation & 20.69 & 16.73 & 18.78 \\
Number missing & 0 & 0 & 0 \\
Median & 5.4 & 5.8 & 5.7 \\
Mode & 4.0 & 5.5 & 5.5 \\
\hline
\end{tabular}


Table A-110. Sample statistics for Sagittal EAM-P Left Final, showing Edgewise sample alone $(n=31)$, MARA + Edgewise alone $(n=33)$ and the total sample $(n=$ 64).

\begin{tabular}{lccc}
\hline \multicolumn{1}{c}{ Statistic } & $\begin{array}{c}\text { Edgewise } \\
\text { Alone }\end{array}$ & $\begin{array}{c}\text { MARA+Edge } \\
\text { Alone }\end{array}$ & $\begin{array}{c}\text { Total } \\
\text { Sample }\end{array}$ \\
\hline Mean & 5.94 & 6.16 & 6.05 \\
Standard deviation & 1.16 & 1.27 & 1.22 \\
Standard error of mean & 0.21 & 0.22 & 0.15 \\
Upper 95\% Mean & 6.36 & 6.61 & 6.36 \\
Lower 95\% Mean & 5.51 & 5.71 & 5.75 \\
Sample size & 31 & 33 & 64 \\
Sample variance & 1.35 & 1.62 & 1.48 \\
Skewness (g1) & 0.00 & 0.71 & 0.43 \\
Kurtosis (g2) & -0.34 & 1.08 & 0.57 \\
Coef Variation & 19.54 & 20.66 & 20.08 \\
Number missing & 0 & 0 & 0 \\
Median & 6.0 & 6.0 & 6.0 \\
Mode & 6.0 & 7.2 & 5.1 \\
\hline
\end{tabular}


Table A-111. Sample statistics for Change in Sagittal EAM-P L, showing Edgewise sample alone $(n=31)$, MARA+Edgewise alone $(n=33)$ and the total sample $(n=64)$.

\begin{tabular}{lccc}
\hline \multicolumn{1}{c}{ Statistic } & $\begin{array}{c}\text { Edgewise } \\
\text { Alone }\end{array}$ & $\begin{array}{c}\text { MARA+Edge } \\
\text { Alone }\end{array}$ & $\begin{array}{c}\text { Total } \\
\text { Sample }\end{array}$ \\
\hline Mean & 0.49 & 0.34 & 0.41 \\
Standard deviation & 0.72 & 0.83 & 0.78 \\
Standard error of mean & 0.13 & 0.14 & 0.10 \\
Upper 95\% Mean & 0.75 & 0.63 & 0.61 \\
Lower 95\% Mean & 0.22 & 0.05 & 0.22 \\
Sample size & 31 & 33 & 64 \\
Sample variance & 0.53 & 0.69 & 0.60 \\
Skewness (g1) & 0.84 & 1.03 & 0.89 \\
Kurtosis (g2) & 0.30 & 4.48 & 2.63 \\
Coef Variation & 148.78 & 244.19 & 189.20 \\
Number missing & 0 & 0 & 0 \\
Median & 0.2 & 0.3 & 0.3 \\
Mode & 0.1 & -0.1 & 0.1 \\
& & One-sample t-test & 4.23 \\
& & & $<0.0001$ \\
\hline
\end{tabular}


Table A-112. Sample statistics for Sagittal EAM-AE Right Initial, showing Edgewise sample alone $(n=31)$, MARA+Edgewise alone $(n=33)$ and the total sample $(n=64)$.

\begin{tabular}{lccc}
\hline \multicolumn{1}{c}{ Statistic } & $\begin{array}{c}\text { Edgewise } \\
\text { Alone }\end{array}$ & $\begin{array}{c}\text { MARA+Edge } \\
\text { Alone }\end{array}$ & $\begin{array}{c}\text { Total } \\
\text { Sample }\end{array}$ \\
\hline Mean & 24.50 & 22.62 & 23.53 \\
Standard deviation & 2.02 & 2.16 & 2.28 \\
Standard error of mean & 0.36 & 0.38 & 0.29 \\
Upper 95\% Mean & 25.24 & 23.39 & 24.10 \\
Lower 95\% Mean & 23.76 & 21.86 & 22.96 \\
Sample size & 31 & 33 & 64 \\
Sample variance & 4.08 & 4.65 & 5.20 \\
Skewness (g1) & 0.45 & -0.04 & 0.06 \\
Kurtosis (g2) & 0.24 & -0.48 & 0.08 \\
Coef Variation & 8.25 & 9.54 & 9.69 \\
Number missing & 0 & 0 & 0 \\
Median & 24.2 & 23.2 & 23.7 \\
Mode & 23.1 & 24.1 & 24.1 \\
\hline
\end{tabular}


Table A-113. Sample statistics for Sagittal EAM-AE Right Final, showing Edgewise sample alone $(n=31)$, MARA+Edgewise alone $(n=33)$ and the total sample $(n=64)$.

\begin{tabular}{lccc}
\hline \multicolumn{1}{c}{ Statistic } & $\begin{array}{c}\text { Edgewise } \\
\text { Alone }\end{array}$ & $\begin{array}{c}\text { MARA+Edge } \\
\text { Alone }\end{array}$ & $\begin{array}{c}\text { Total } \\
\text { Sample }\end{array}$ \\
\hline Mean & 24.92 & 23.23 & 24.05 \\
Standard deviation & 2.17 & 1.97 & 2.22 \\
Standard error of mean & 0.39 & 0.34 & 0.28 \\
Upper 95\% Mean & 25.71 & 23.93 & 24.60 \\
Lower 95\% Mean & 24.12 & 22.53 & 23.49 \\
Sample size & 31 & 33 & 64 \\
Sample variance & 4.72 & 3.89 & 4.94 \\
Skewness (g1) & 0.00 & -0.47 & -0.06 \\
Kurtosis (g2) & -0.30 & -0.33 & -0.02 \\
Coef Variation & 8.72 & 8.49 & 9.24 \\
Number missing & 0 & 0 & 0 \\
Median & 24.8 & 23.5 & 24.3 \\
Mode & 24.4 & 22.6 & 22.6 \\
\hline
\end{tabular}


Table A-114. Sample statistics for Change in Sagittal EAM-AE R, showing Edgewise sample alone $(n=31)$, MARA+Edgewise alone $(n=33)$ and the total sample $(n=64)$.

\begin{tabular}{lccc}
\hline \multicolumn{1}{c}{ Statistic } & $\begin{array}{c}\text { Edgewise } \\
\text { Alone }\end{array}$ & $\begin{array}{c}\text { MARA+Edge } \\
\text { Alone }\end{array}$ & $\begin{array}{c}\text { Total } \\
\text { Sample }\end{array}$ \\
\hline Mean & 0.42 & 0.61 & 0.52 \\
Standard deviation & 1.19 & 1.34 & 1.26 \\
Standard error of mean & 0.21 & 0.23 & 0.16 \\
Upper 95\% Mean & 0.85 & 1.09 & 0.83 \\
Lower 95\% Mean & -0.02 & 0.14 & 0.20 \\
Sample size & 31 & 33 & 64 \\
Sample variance & 1.41 & 1.80 & 1.60 \\
Skewness (g1) & 0.37 & -0.14 & 0.09 \\
Kurtosis (g2) & 2.36 & -0.36 & 0.45 \\
Coef Variation & 285.43 & 219.40 & 244.39 \\
Number missing & 0 & 0 & 0 \\
Median & 0.2 & 0.5 & 0.4 \\
Mode & 0.2 & -0.5 & 0.0 \\
& & One-sample t-test & 3.27 \\
& & & 0.0017 \\
\hline
\end{tabular}


Table A-115. Sample statistics for Sagittal EAM-C Right Initial, showing Edgewise sample alone $(n=31)$, MARA+Edgewise alone $(n=33)$ and the total sample $(n=64)$.

\begin{tabular}{lccc}
\hline \multicolumn{1}{c}{ Statistic } & $\begin{array}{c}\text { Edgewise } \\
\text { Alone }\end{array}$ & $\begin{array}{c}\text { MARA+Edge } \\
\text { Alone }\end{array}$ & $\begin{array}{c}\text { Total } \\
\text { Sample }\end{array}$ \\
\hline Mean & 13.72 & 12.98 & 13.34 \\
Standard deviation & 1.34 & 1.49 & 1.46 \\
Standard error of mean & 0.24 & 0.26 & 0.18 \\
Upper 95\% Mean & 14.21 & 13.51 & 13.70 \\
Lower 95\% Mean & 13.23 & 12.45 & 12.98 \\
Sample size & 31 & 33 & 64 \\
Sample variance & 1.79 & 2.23 & 2.12 \\
Skewness (g1) & 0.98 & -0.07 & 0.23 \\
Kurtosis (g2) & 0.94 & -0.51 & 0.40 \\
Coef Variation & 9.75 & 11.50 & 10.92 \\
Number missing & 0 & 0 & 0 \\
Median & 13.4 & 13.0 & 13.2 \\
Mode & 12.7 & 11.6 & 13.0 \\
\hline
\end{tabular}


Table A-116. Sample statistics for Sagittal EAM-C Right Final, showing Edgewise sample alone $(n=31)$, MARA+Edgewise alone $(n=33)$ and the total sample $(n=$ 64).

\begin{tabular}{lccc}
\hline \multicolumn{1}{c}{ Statistic } & $\begin{array}{c}\text { Edgewise } \\
\text { Alone }\end{array}$ & $\begin{array}{c}\text { MARA+Edge } \\
\text { Alone }\end{array}$ & $\begin{array}{c}\text { Total } \\
\text { Sample }\end{array}$ \\
\hline Mean & 14.00 & 13.11 & 13.54 \\
Standard deviation & 1.51 & 1.43 & 1.52 \\
Standard error of mean & 0.27 & 0.25 & 0.19 \\
Upper 95\% Mean & 14.56 & 13.62 & 13.92 \\
Lower 95\% Mean & 13.45 & 12.60 & 13.16 \\
Sample size & 31 & 33 & 64 \\
Sample variance & 2.27 & 2.05 & 2.32 \\
Skewness (g1) & 0.24 & -0.52 & -0.06 \\
Kurtosis (g2) & 0.93 & 0.46 & 0.87 \\
Coef Variation & 10.76 & 10.92 & 11.25 \\
Number missing & 0 & 0 & 0 \\
Median & 13.7 & 13.2 & 13.6 \\
Mode & 13.0 & 13.6 & 13.0 \\
\hline
\end{tabular}


Table A-117. Sample statistics for Change in Sagittal EAM-C R, showing Edgewise sample alone $(n=31)$, MARA+Edgewise alone $(n=33)$ and the total sample $(n=64)$.

\begin{tabular}{lccc}
\hline \multicolumn{1}{c}{ Statistic } & $\begin{array}{c}\text { Edgewise } \\
\text { Alone }\end{array}$ & $\begin{array}{c}\text { MARA+Edge } \\
\text { Alone }\end{array}$ & $\begin{array}{c}\text { Total } \\
\text { Sample }\end{array}$ \\
\hline Mean & 0.28 & 0.13 & 0.20 \\
Standard deviation & 0.87 & 1.08 & 0.98 \\
Standard error of mean & 0.16 & 0.19 & 0.12 \\
Upper 95\% Mean & 0.60 & 0.51 & 0.45 \\
Lower 95\% Mean & -0.04 & -0.25 & -0.04 \\
Sample size & 31 & 33 & 64 \\
Sample variance & 0.76 & 1.17 & 0.96 \\
Skewness (g1) & 0.71 & 0.94 & 0.80 \\
Kurtosis (g2) & 2.98 & 2.58 & 2.49 \\
Coef Variation & 306.31 & 829.78 & 478.63 \\
Number missing & 0 & 0 & 0 \\
Median & 0.3 & -0.1 & 0.3 \\
Mode & 0.3 & -0.2 & 0.3 \\
& & One-sample t-test & 1.67 \\
& & & 0.0996 \\
& & &
\end{tabular}


Table A-118. Sample statistics for Sagittal EAM-P Right Initial, showing Edgewise sample alone $(n=31)$, MARA+Edgewise alone $(n=33)$ and the total sample $(n=64)$.

\begin{tabular}{lccc}
\hline \multicolumn{1}{c}{ Statistic } & $\begin{array}{c}\text { Edgewise } \\
\text { Alone }\end{array}$ & $\begin{array}{c}\text { MARA+Edge } \\
\text { Alone }\end{array}$ & $\begin{array}{c}\text { Total } \\
\text { Sample }\end{array}$ \\
\hline Mean & 6.10 & 5.85 & 5.97 \\
Standard deviation & 1.28 & 0.94 & 1.11 \\
Standard error of mean & 0.23 & 0.16 & 0.14 \\
Upper 95\% Mean & 6.57 & 6.18 & 6.25 \\
Lower 95\% Mean & 5.63 & 5.52 & 5.69 \\
Sample size & 31 & 33 & 64 \\
Sample variance & 1.64 & 0.88 & 1.24 \\
Skewness (g1) & 1.03 & 0.56 & 0.99 \\
Kurtosis (g2) & 1.42 & 1.11 & 1.78 \\
Coef Variation & 20.98 & 16.00 & 18.65 \\
Number missing & 0 & 0 & 0 \\
Median & 6.1 & 5.9 & 6.0 \\
Mode & 5.3 & 6.0 & 5.5 \\
\hline
\end{tabular}


Table A-119. Sample statistics for Sagittal EAM-P Right Final, showing Edgewise sample alone $(n=31)$, MARA + Edgewise alone $(n=33)$ and the total sample $(n=$ 64).

\begin{tabular}{lccc}
\hline \multicolumn{1}{c}{ Statistic } & $\begin{array}{c}\text { Edgewise } \\
\text { Alone }\end{array}$ & $\begin{array}{c}\text { MARA+Edge } \\
\text { Alone }\end{array}$ & $\begin{array}{c}\text { Total } \\
\text { Sample }\end{array}$ \\
\hline Mean & 6.11 & 6.04 & 6.08 \\
Standard deviation & 1.27 & 0.85 & 1.07 \\
Standard error of mean & 0.23 & 0.15 & 0.13 \\
Upper 95\% Mean & 6.58 & 6.34 & 6.34 \\
Lower 95\% Mean & 5.64 & 5.74 & 5.81 \\
Sample size & 31 & 33 & 64 \\
Sample variance & 1.62 & 0.73 & 1.14 \\
Skewness (g1) & 0.53 & -0.15 & 0.41 \\
Kurtosis (g2) & 0.24 & 0.09 & 0.63 \\
Coef Variation & 20.82 & 14.10 & 17.58 \\
Number missing & 0 & 0 & 0 \\
Median & 6.0 & 6.1 & 6.1 \\
Mode & 4.3 & 5.6 & 6.2 \\
\hline
\end{tabular}


Table A-120. Sample statistics for Change in EAM P-R, showing Edgewise sample alone $(n=31)$, MARA+Edgewise alone $(n=33)$ and the total sample $(n=64)$.

\begin{tabular}{lccc}
\hline \multicolumn{1}{c}{ Statistic } & $\begin{array}{c}\text { Edgewise } \\
\text { Alone }\end{array}$ & $\begin{array}{c}\text { MARA+Edge } \\
\text { Alone }\end{array}$ & $\begin{array}{c}\text { Total } \\
\text { Sample }\end{array}$ \\
\hline Mean & 0.01 & 0.19 & 0.10 \\
Standard deviation & 0.64 & 0.62 & 0.63 \\
Standard error of mean & 0.11 & 0.11 & 0.08 \\
Upper 95\% Mean & 0.24 & 0.41 & 0.26 \\
Lower 95\% Mean & -0.22 & -0.03 & -0.05 \\
Sample size & 31 & 33 & 64 \\
Sample variance & 0.40 & 0.38 & 0.39 \\
Skewness (g1) & -1.98 & -0.01 & -0.96 \\
Kurtosis (g2) & 7.21 & 0.43 & 3.95 \\
Coef Variation & $6,564.47$ & 322.99 & 608.40 \\
Number missing & 0 & 0 & 0 \\
Median & 0.0 & 0.2 & 0.2 \\
Mode & -0.1 & 0.2 & 0.0 \\
& & One-sample t-test & 1.31 \\
& & & 0.1933 \\
\hline
\end{tabular}


Table A-121. Sample statistics for Sagittal ATA Left Initial, showing Edgewise sample alone $(n=31)$, MARA+Edgewise alone $(n=33)$ and the total sample $(n=$ 64).

\begin{tabular}{lccc}
\hline \multicolumn{1}{c}{ Statistic } & $\begin{array}{c}\text { Edgewise } \\
\text { Alone }\end{array}$ & $\begin{array}{c}\text { MARA+Edge } \\
\text { Alone }\end{array}$ & $\begin{array}{c}\text { Total } \\
\text { Sample }\end{array}$ \\
\hline Mean & 36.76 & 43.95 & 40.47 \\
Standard deviation & 5.64 & 7.71 & 7.64 \\
Standard error of mean & 1.01 & 1.34 & 0.96 \\
Upper 95\% Mean & 38.83 & 46.68 & 42.38 \\
Lower 95\% Mean & 34.69 & 41.22 & 38.56 \\
Sample size & 31 & 33 & 64 \\
Sample variance & 31.81 & 59.38 & 58.43 \\
Skewness (g1) & -0.62 & 0.20 & 0.32 \\
Kurtosis (g2) & 0.03 & -0.02 & 0.45 \\
Coef Variation & 15.34 & 17.53 & 18.89 \\
Number missing & 0 & 0 & 0 \\
Median & 38.3 & 44.3 & 40.3 \\
Mode & 26.6 & 44.3 & 39.7 \\
\hline
\end{tabular}


Table A-122. Sample statistics for Sagittal ATA Left Final, showing Edgewise sample alone $(n=31)$, MARA+Edgewise alone $(n=33)$ and the total sample $(n=$ 64).

\begin{tabular}{lccc}
\hline \multicolumn{1}{c}{ Statistic } & $\begin{array}{c}\text { Edgewise } \\
\text { Alone }\end{array}$ & $\begin{array}{c}\text { MARA+Edge } \\
\text { Alone }\end{array}$ & $\begin{array}{c}\text { Total } \\
\text { Sample }\end{array}$ \\
\hline Mean & 37.92 & 41.83 & 39.94 \\
Standard deviation & 6.51 & 7.84 & 7.43 \\
Standard error of mean & 1.17 & 1.36 & 0.93 \\
Upper 95\% Mean & 40.30 & 44.61 & 41.79 \\
Lower 95\% Mean & 35.53 & 39.05 & 38.08 \\
Sample size & 31 & 33 & 64 \\
Sample variance & 42.35 & 61.40 & 55.25 \\
Skewness (g1) & 0.05 & 0.88 & 0.66 \\
Kurtosis (g2) & 0.38 & 2.18 & 1.88 \\
Coef Variation & 17.16 & 18.73 & 18.61 \\
Number missing & 0 & 0 & 0 \\
Median & 37.5 & 41.5 & 39.7 \\
Mode & 37.1 & 37.9 & 37.1 \\
\hline
\end{tabular}


Table A-123. Sample statistics for Change in Sagittal ATA L, showing Edgewise sample alone $(n=31)$, MARA + Edgewise alone $(n=33)$ and the total sample $(n=$ 64).

\begin{tabular}{lccc}
\hline \multicolumn{1}{c}{ Statistic } & $\begin{array}{c}\text { Edgewise } \\
\text { Alone }\end{array}$ & $\begin{array}{c}\text { MARA+Edge } \\
\text { Alone }\end{array}$ & $\begin{array}{c}\text { Total } \\
\text { Sample }\end{array}$ \\
\hline Mean & 1.15 & -2.12 & -0.53 \\
Standard deviation & 4.34 & 4.32 & 4.60 \\
Standard error of mean & 0.78 & 0.75 & 0.57 \\
Upper 95\% Mean & 2.75 & -0.59 & 0.62 \\
Lower 95\% Mean & -0.44 & -3.65 & -1.68 \\
Sample size & 31 & 33 & 64 \\
Sample variance & 18.82 & 18.66 & 21.16 \\
Skewness (g1) & -0.75 & -0.02 & -0.29 \\
Kurtosis (g2) & 0.55 & -0.47 & -0.51 \\
Coef Variation & 375.63 & -203.94 & -863.29 \\
Number missing & 0 & 0 & 0 \\
Median & 1.4 & -1.4 & -0.1 \\
Mode & 3.7 & -6.8 & -5.9 \\
& & P-value (two tail) & 0.3576 \\
\hline
\end{tabular}


Table A-124. Sample statistics for Sagittal CHA Left Initial, showing Edgewise sample alone $(n=31)$, MARA+Edgewise alone $(n=33)$ and the total sample $(n=$ 64).

\begin{tabular}{lccc}
\hline \multicolumn{1}{c}{ Statistic } & $\begin{array}{c}\text { Edgewise } \\
\text { Alone }\end{array}$ & $\begin{array}{c}\text { MARA+Edge } \\
\text { Alone }\end{array}$ & $\begin{array}{c}\text { Total } \\
\text { Sample }\end{array}$ \\
\hline Mean & 141.59 & 141.18 & 141.38 \\
Standard deviation & 8.76 & 8.43 & 8.52 \\
Standard error of mean & 1.57 & 1.47 & 1.07 \\
Upper 95\% Mean & 144.80 & 144.17 & 143.51 \\
Lower 95\% Mean & 138.38 & 138.20 & 139.25 \\
Sample size & 31 & 33 & 64 \\
Sample variance & 76.69 & 71.03 & 72.64 \\
Skewness (g1) & -0.48 & 0.17 & -0.16 \\
Kurtosis (g2) & 1.31 & 0.06 & 0.52 \\
Coef Variation & 6.18 & 5.97 & 6.03 \\
Number missing & 0 & 0 & 0 \\
Median & 142.4 & 141.9 & 142.2 \\
Mode & 135.8 & 134.1 & 135.8 \\
\hline
\end{tabular}


Table A-125. Sample statistics for Sagittal CHA Left Final, showing Edgewise sample alone $(n=31)$, MARA + Edgewise alone $(n=33)$ and the total sample $(n=$ 64).

\begin{tabular}{lccc}
\hline \multicolumn{1}{c}{ Statistic } & $\begin{array}{c}\text { Edgewise } \\
\text { Alone }\end{array}$ & $\begin{array}{c}\text { MARA+Edge } \\
\text { Alone }\end{array}$ & $\begin{array}{c}\text { Total } \\
\text { Sample }\end{array}$ \\
\hline Mean & 135.11 & 133.47 & 134.27 \\
Standard deviation & 9.86 & 13.19 & 11.64 \\
Standard error of mean & 1.77 & 2.30 & 1.45 \\
Upper 95\% Mean & 138.73 & 138.15 & 137.17 \\
Lower 95\% Mean & 131.50 & 128.80 & 131.36 \\
Sample size & 31 & 33 & 64 \\
Sample variance & 97.28 & 173.98 & 135.38 \\
Skewness (g1) & 0.08 & -0.45 & -0.36 \\
Kurtosis (g2) & -0.44 & -0.46 & -0.20 \\
Coef Variation & 7.30 & 9.88 & 8.67 \\
Number missing & 0 & 0 & 0 \\
Median & 135.8 & 135.6 & 135.7 \\
Mode & 122.4 &. & 128.9 \\
\hline
\end{tabular}


Table A-126. Sample statistics for Change in Sagittal CHA L, showing Edgewise sample alone $(n=31)$, MARA + Edgewise alone $(n=33)$ and the total sample $(n=$ 64).

\begin{tabular}{lccc}
\hline \multicolumn{1}{c}{ Statistic } & $\begin{array}{c}\text { Edgewise } \\
\text { Alone }\end{array}$ & $\begin{array}{c}\text { MARA+Edge } \\
\text { Alone }\end{array}$ & $\begin{array}{c}\text { Total } \\
\text { Sample }\end{array}$ \\
\hline Mean & & & \\
Standard deviation & -6.48 & -7.71 & -7.11 \\
Standard error of mean & 8.39 & 12.08 & 10.39 \\
Upper 95\% Mean & 1.51 & 2.10 & 1.30 \\
Lower 95\% Mean & -3.40 & -3.43 & -4.52 \\
Sample size & -9.56 & -12.00 & -9.71 \\
Sample variance & 31 & 33 & 64 \\
Skewness (g1) & 70.42 & 145.94 & 108.04 \\
Kurtosis (g2) & -0.86 & -0.83 & -0.92 \\
Coef Variation & 1.75 & 1.35 & 1.83 \\
Number missing & -129.55 & -156.64 & -146.11 \\
Median & 0 & 0 & 0 \\
Mode & -6.4 & -6.5 & -6.5 \\
& -0.8 & P-value (two tail) & $<0.0001$ \\
\hline
\end{tabular}


Table A-127. Sample statistics for Sagittal ATA Right Initial, showing Edgewise sample alone $(n=31)$, MARA+Edgewise alone $(n=33)$ and the total sample $(n=$ 64).

\begin{tabular}{lccc}
\hline \multicolumn{1}{c}{ Statistic } & $\begin{array}{c}\text { Edgewise } \\
\text { Alone }\end{array}$ & $\begin{array}{c}\text { MARA+Edge } \\
\text { Alone }\end{array}$ & $\begin{array}{c}\text { Total } \\
\text { Sample }\end{array}$ \\
\hline Mean & 36.71 & 44.79 & 40.88 \\
Standard deviation & 5.83 & 6.85 & 7.52 \\
Standard error of mean & 1.05 & 1.19 & 0.94 \\
Upper 95\% Mean & 38.85 & 47.22 & 42.76 \\
Lower 95\% Mean & 34.57 & 42.36 & 39.00 \\
Sample size & 31 & 33 & 64 \\
Sample variance & 33.98 & 46.96 & 56.62 \\
Skewness (g1) & -0.27 & -0.16 & 0.06 \\
Kurtosis (g2) & 0.60 & -0.79 & -0.31 \\
Coef Variation & 15.88 & 15.30 & 18.4 \\
Number missing & 0 & 0 & 0 \\
Median & 36.7 & 45.5 & 40.4 \\
Mode & 37.8 & 45.5 & 36.6 \\
\hline
\end{tabular}


Table A-128. Sample statistics for Sagittal ATA Right Final, showing Edgewise sample alone $(n=31)$, MARA+Edgewise alone $(n=33)$ and the total sample $(n=$ 64).

\begin{tabular}{lccc}
\hline \multicolumn{1}{c}{ Statistic } & $\begin{array}{c}\text { Edgewise } \\
\text { Alone }\end{array}$ & $\begin{array}{c}\text { MARA+Edge } \\
\text { Alone }\end{array}$ & $\begin{array}{c}\text { Total } \\
\text { Sample }\end{array}$ \\
\hline Mean & 38.24 & 42.79 & 40.59 \\
Standard deviation & 5.46 & 6.94 & 6.62 \\
Standard error of mean & 0.98 & 1.21 & 0.83 \\
Upper 95\% Mean & 40.24 & 45.25 & 42.24 \\
Lower 95\% Mean & 36.24 & 40.33 & 38.93 \\
Sample size & 31 & 33 & 64 \\
Sample variance & 29.80 & 48.12 & 43.88 \\
Skewness (g1) & -0.32 & 0.01 & 0.14 \\
Kurtosis (g2) & -0.30 & -0.89 & -0.39 \\
Coef Variation & 14.28 & 16.21 & 16.32 \\
Number missing & 0 & 0 & 0 \\
Median & 38.6 & 43.1 & 40.2 \\
Mode & 38.6 & 50.7 & 35.4 \\
\hline
\end{tabular}


Table A-129. Sample statistics for Change in Sagittal ATA R, showing Edgewise sample alone $(n=31)$, MARA + Edgewise alone $(n=33)$ and the total sample $(n=$ 64).

\begin{tabular}{lccc}
\hline \multicolumn{1}{c}{ Statistic } & $\begin{array}{c}\text { Edgewise } \\
\text { Alone }\end{array}$ & $\begin{array}{c}\text { MARA+Edge } \\
\text { Alone }\end{array}$ & $\begin{array}{c}\text { Total } \\
\text { Sample }\end{array}$ \\
Mean & 1.53 & -2.01 & -0.29 \\
Standard deviation & 4.00 & 4.13 & 4.41 \\
Standard error of mean & 0.72 & 0.72 & 0.55 \\
Upper 95\% Mean & 3.00 & -0.54 & 0.81 \\
Lower 95\% Mean & 0.06 & -3.47 & -1.39 \\
Sample size & 31 & 33 & 64 \\
Sample variance & 16.01 & 17.07 & 19.47 \\
Skewness (g1) & -0.26 & -0.53 & -0.33 \\
Kurtosis (g2) & 0.34 & 2.17 & 0.97 \\
Coef Variation & 261.10 & -205.97 & $-1,510.15$ \\
Number missing & 0 & 0 & 0 \\
Median & 1.8 & -1.9 & -0.1 \\
Mode & -2.4 & -6.0 & 1.8 \\
& & P-value (two tail) & 0.5981 \\
\hline
\end{tabular}


Table A-130. Sample statistics for Sagittal CHA Right Initial, showing Edgewise sample alone $(n=31)$, MARA + Edgewise alone $(n=33)$ and the total sample $(n=$ 64).

\begin{tabular}{lccc}
\hline \multicolumn{1}{c}{ Statistic } & $\begin{array}{c}\text { Edgewise } \\
\text { Alone }\end{array}$ & $\begin{array}{c}\text { MARA+Edge } \\
\text { Alone }\end{array}$ & $\begin{array}{c}\text { Total } \\
\text { Sample }\end{array}$ \\
\hline Mean & 143.99 & 137.92 & 140.86 \\
Standard deviation & 6.68 & 9.03 & 8.49 \\
Standard error of mean & 1.20 & 1.57 & 1.06 \\
Upper 95\% Mean & 146.44 & 141.12 & 142.98 \\
Lower 95\% Mean & 141.54 & 134.71 & 138.74 \\
Sample size & 31 & 33 & 64 \\
Sample variance & 44.58 & 81.63 & 72.06 \\
Skewness (g1) & -0.45 & -0.42 & -0.63 \\
Kurtosis (g2) & 0.08 & -0.46 & 0.05 \\
Coef Variation & 4.64 & 6.55 & 6.03 \\
Number missing & 0 & 0 & 0 \\
Median & 143.6 & 139.5 & 142.2 \\
Mode & 143.6 & 134.6 & 139.5 \\
\hline
\end{tabular}


Table A-131. Sample statistics for Sagittal CHA Right Final, showing Edgewise sample alone $(n=31)$, MARA+Edgewise alone $(n=33)$ and the total sample $(n=$ 64).

\begin{tabular}{lccc}
\hline \multicolumn{1}{c}{ Statistic } & $\begin{array}{c}\text { Edgewise } \\
\text { Alone }\end{array}$ & $\begin{array}{c}\text { MARA+Edge } \\
\text { Alone }\end{array}$ & $\begin{array}{c}\text { Total } \\
\text { Sample }\end{array}$ \\
\hline Mean & 138.69 & 131.83 & 135.15 \\
Standard deviation & 8.58 & 12.17 & 11.06 \\
Standard error of mean & 1.54 & 2.12 & 1.38 \\
Upper 95\% Mean & 141.84 & 136.14 & 137.92 \\
Lower 95\% Mean & 135.55 & 127.51 & 132.39 \\
Sample size & 31 & 33 & 64 \\
Sample variance & 73.60 & 148.20 & 122.29 \\
Skewness (g1) & -0.26 & -0.20 & -0.47 \\
Kurtosis (g2) & 1.62 & -0.98 & -0.14 \\
Coef Variation & 6.19 & 9.23 & 8.18 \\
Number missing & 0 & 0 & 0 \\
Median & 140.5 & 134.5 & 138.1 \\
Mode & 143.8 & 120.2 & 134.5 \\
\hline
\end{tabular}


Table A-132. Sample statistics for Change in Sagittal CHA R, showing Edgewise sample alone $(n=31)$, MARA + Edgewise alone $(n=33)$ and the total sample $(n=$ 64).

\begin{tabular}{lccc}
\hline \multicolumn{1}{c}{ Statistic } & $\begin{array}{c}\text { Edgewise } \\
\text { Alone }\end{array}$ & $\begin{array}{c}\text { MARA+Edge } \\
\text { Alone }\end{array}$ & $\begin{array}{c}\text { Total } \\
\text { Sample }\end{array}$ \\
\hline Mean & -5.30 & -6.09 & -5.71 \\
Standard deviation & 6.12 & 10.65 & 8.69 \\
Standard error of mean & 1.10 & 1.85 & 1.09 \\
Upper 95\% Mean & -3.06 & -2.32 & -3.54 \\
Lower 95\% Mean & -7.54 & -9.87 & -7.88 \\
Sample size & 31 & 33 & 64 \\
Sample variance & 37.45 & 113.40 & 75.59 \\
Skewness (g1) & -0.22 & -0.33 & -0.41 \\
Kurtosis (g2) & -0.27 & 0.81 & 1.52 \\
Coef Variation & -115.47 & -174.83 & -152.32 \\
Number missing & 0 & 0 & 0 \\
Median & -4.2 & -6.4 & -5.3 \\
Mode & -4.2 & -7.5 & -4.2 \\
& & One-sample t-test & 5.25 \\
& & & $<0.0001$ \\
\hline
\end{tabular}


Table A-133. Sample statistics for Sagittal ACP Left Initial, showing Edgewise sample alone $(n=31)$, MARA+Edgewise alone $(n=33)$ and the total sample $(n=$ 64).

\begin{tabular}{lccc}
\hline \multicolumn{1}{c}{ Statistic } & $\begin{array}{c}\text { Edgewise } \\
\text { Alone }\end{array}$ & $\begin{array}{c}\text { MARA+Edge } \\
\text { Alone }\end{array}$ & $\begin{array}{c}\text { Total } \\
\text { Sample }\end{array}$ \\
\hline Mean & 48.65 & 42.69 & 45.58 \\
Standard deviation & 12.35 & 9.57 & 11.32 \\
Standard error of mean & 2.22 & 1.67 & 1.42 \\
Upper 95\% Mean & 53.19 & 46.08 & 48.41 \\
Lower 95\% Mean & 44.12 & 39.30 & 42.75 \\
Sample size & 31 & 33 & 64 \\
Sample variance & 152.59 & 91.57 & 128.20 \\
Skewness (g1) & 0.70 & 0.27 & 0.69 \\
Kurtosis (g2) & 0.00 & -0.05 & 0.47 \\
Coef Variation & 25.39 & 22.42 & 24.84 \\
Number missing & 0 & & 0 \\
Median & 45.8 & 43.1 & 43.6 \\
Mode & 36.9 & 35.6 & 46.7 \\
\hline
\end{tabular}


Table A-134. Sample statistics for Sagittal ACP Left Final, showing Edgewise sample alone $(n=31)$, MARA + Edgewise alone $(n=33)$ and the total sample $(n=$ 64).

\begin{tabular}{lccc}
\hline \multicolumn{1}{c}{ Statistic } & $\begin{array}{c}\text { Edgewise } \\
\text { Alone }\end{array}$ & $\begin{array}{c}\text { MARA+Edge } \\
\text { Alone }\end{array}$ & $\begin{array}{c}\text { Total } \\
\text { Sample }\end{array}$ \\
\hline Mean & 50.20 & 42.11 & 46.03 \\
Standard deviation & 11.71 & 9.55 & 11.32 \\
Standard error of mean & 2.10 & 1.66 & 1.41 \\
Upper 95\% Mean & 54.49 & 45.49 & 48.85 \\
Lower 95\% Mean & 45.90 & 38.72 & 43.20 \\
Sample size & 31 & 33 & 64 \\
Sample variance & 137.01 & 91.12 & 128.13 \\
Skewness (g1) & 0.40 & 1.26 & 0.77 \\
Kurtosis (g2) & -0.28 & 1.74 & 0.06 \\
Coef Variation & 23.32 & 22.67 & 24.59 \\
Number missing & 0 & 0 & 0 \\
Median & 49.5 & 39.8 & 44.7 \\
Mode &. & 33.8 & 33.8 \\
\hline
\end{tabular}


Table A-135. Sample statistics for Change in ACP L, showing Edgewise sample alone $(n=31)$, MARA+Edgewise alone $(n=33)$ and the total sample $(n=64)$.

\begin{tabular}{lccc}
\hline \multicolumn{1}{c}{ Statistic } & $\begin{array}{c}\text { Edgewise } \\
\text { Alone }\end{array}$ & $\begin{array}{c}\text { MARA+Edge } \\
\text { Alone }\end{array}$ & $\begin{array}{c}\text { Total } \\
\text { Sample }\end{array}$ \\
\hline Mean & 1.54 & -0.58 & 0.45 \\
Standard deviation & 8.98 & 6.00 & 7.60 \\
Standard error of mean & 1.61 & 1.04 & 0.95 \\
Upper 95\% Mean & 4.83 & 1.54 & 2.34 \\
Lower 95\% Mean & -1.75 & -2.71 & -1.45 \\
Sample size & 31 & 33 & 64 \\
Sample variance & 80.57 & 36.03 & 57.82 \\
Skewness (g1) & -1.18 & 0.01 & -0.73 \\
Kurtosis (g2) & 2.63 & -0.50 & 1.89 \\
Coef Variation & 582.13 & $-1,026.35$ & $1,707.49$ \\
Number missing & 0 & 0 & 0 \\
Median & 1.1 & 0.3 & 0.8 \\
Mode & -0.9 & -9.1 & -0.9 \\
& & One-sample t-test & 0.47 \\
& & & 0.6410 \\
\hline
\end{tabular}


Table A-136. Sample statistics for Sagittal AAE Left Initial, showing Edgewise sample alone $(n=31)$, MARA+Edgewise alone $(n=33)$ and the total sample $(n=$ 64).

\begin{tabular}{lccc}
\hline \multicolumn{1}{c}{ Statistic } & $\begin{array}{c}\text { Edgewise } \\
\text { Alone }\end{array}$ & $\begin{array}{c}\text { MARA+Edge } \\
\text { Alone }\end{array}$ & $\begin{array}{c}\text { Total } \\
\text { Sample }\end{array}$ \\
\hline Mean & 86.10 & 89.88 & 88.05 \\
Standard deviation & 16.34 & 19.02 & 17.74 \\
Standard error of mean & 2.94 & 3.31 & 2.22 \\
Upper 95\% Mean & 92.10 & 96.63 & 92.48 \\
Lower 95\% Mean & 80.11 & 83.14 & 83.62 \\
Sample size & 31 & 33 & 64 \\
Sample variance & 267.11 & 361.77 & 314.58 \\
Skewness (g1) & 0.30 & 0.06 & 0.19 \\
Kurtosis (g2) & -0.59 & 0.30 & -0.06 \\
Coef Variation & 18.98 & 21.16 & 20.14 \\
Number missing & 0 & 0 & 0 \\
Median & 84.5 & 88.2 & 86.0 \\
Mode &. &. & 80.9 \\
\hline
\end{tabular}


Table A-137. Sample statistics for Sagittal AAE Left Final, showing Edgewise sample alone $(n=31)$, MARA+Edgewise alone $(n=33)$ and the total sample $(n=$ 64).

\begin{tabular}{lccc}
\hline \multicolumn{1}{c}{ Statistic } & $\begin{array}{c}\text { Edgewise } \\
\text { Alone }\end{array}$ & $\begin{array}{c}\text { MARA+Edge } \\
\text { Alone }\end{array}$ & $\begin{array}{c}\text { Total } \\
\text { Sample }\end{array}$ \\
\hline Mean & 97.19 & 99.73 & 98.50 \\
Standard deviation & 19.30 & 27.06 & 23.47 \\
Standard error of mean & 3.47 & 4.71 & 2.93 \\
Upper 95\% Mean & 104.27 & 109.33 & 104.37 \\
Lower 95\% Mean & 90.12 & 90.13 & 92.64 \\
Sample size & 31 & 33 & 64 \\
Sample variance & 372.37 & 732.51 & 551.02 \\
Skewness (g1) & 0.35 & 0.92 & 0.84 \\
Kurtosis (g2) & 0.20 & 1.26 & 1.43 \\
Coef Variation & 19.85 & 27.14 & 23.83 \\
Number missing & 0 & 0 & 0 \\
Median & 97.1 & 95.3 & 96.2 \\
Mode & 112.9 &. & 81.2 \\
\hline
\end{tabular}


Table A-138. Sample statistics for Change in Sagittal AAE L, showing Edgewise sample alone $(n=31)$, MARA + Edgewise alone $(n=33)$ and the total sample $(n=$ 64).

\begin{tabular}{lccc}
\hline \multicolumn{1}{c}{ Statistic } & $\begin{array}{c}\text { Edgewise } \\
\text { Alone }\end{array}$ & $\begin{array}{c}\text { MARA+Edge } \\
\text { Alone }\end{array}$ & $\begin{array}{c}\text { Total } \\
\text { Sample }\end{array}$ \\
\hline Mean & 11.09 & & \\
Standard deviation & 11.93 & 16.14 & 10.45 \\
Standard error of mean & 2.14 & 2.81 & 14.16 \\
Upper 95\% Mean & 15.46 & 15.57 & 1.77 \\
Lower 95\% Mean & 6.72 & 4.12 & 13.98 \\
Sample size & 31 & 33 & 6.91 \\
Sample variance & 142.22 & 260.48 & 64 \\
Skewness (g1) & -0.16 & 0.20 & 200.43 \\
Kurtosis (g2) & -0.27 & -0.59 & 0.06 \\
Coef Variation & 107.53 & 163.93 & -0.39 \\
Number missing & 0 & 0 & 135.50 \\
Median & 11.8 & 8.4 & 0 \\
Mode & 2.8 & P-value (two tail) & $<0.0001$ \\
& & & 9.2 \\
& & & 2.8 \\
& & & 5.90 \\
& & &
\end{tabular}


Table A-139. Sample statistics for Sagittal AGF Left Initial, showing Edgewise sample alone $(n=31)$, MARA+Edgewise alone $(n=33)$ and the total sample $(n=$ 64).

\begin{tabular}{lccc}
\hline \multicolumn{1}{c}{ Statistic } & $\begin{array}{c}\text { Edgewise } \\
\text { Alone }\end{array}$ & $\begin{array}{c}\text { MARA+Edge } \\
\text { Alone }\end{array}$ & $\begin{array}{c}\text { Total } \\
\text { Sample }\end{array}$ \\
\hline Mean & 69.95 & 82.42 & 76.38 \\
Standard deviation & 14.31 & 15.78 & 16.23 \\
Standard error of mean & 2.57 & 2.75 & 2.03 \\
Upper 95\% Mean & 75.19 & 88.02 & 80.43 \\
Lower 95\% Mean & 64.70 & 76.83 & 72.32 \\
Sample size & 31 & 33 & 64 \\
Sample variance & 204.71 & 248.89 & 263.40 \\
Skewness (g1) & -0.67 & 0.30 & 0.02 \\
Kurtosis (g2) & 0.47 & -0.25 & 0.51 \\
Coef Variation & 20.46 & 19.14 & 21.25 \\
Number missing & 0 & 0 & 0 \\
Median & 72.9 & 82.1 & 76.2 \\
Mode & 69.2 & 70.4 & 69.2 \\
\hline
\end{tabular}


Table A-140. Sample statistics for Sagittal AGF Left Final, showing Edgewise sample alone $(n=31)$, MARA+Edgewise alone $(n=33)$ and the total sample $(n=$ 64).

\begin{tabular}{lccc}
\hline \multicolumn{1}{c}{ Statistic } & $\begin{array}{c}\text { Edgewise } \\
\text { Alone }\end{array}$ & $\begin{array}{c}\text { MARA+Edge } \\
\text { Alone }\end{array}$ & $\begin{array}{c}\text { Total } \\
\text { Sample }\end{array}$ \\
\hline Mean & 77.87 & 81.86 & 79.93 \\
Standard deviation & 17.03 & 17.37 & 17.19 \\
Standard error of mean & 3.06 & 3.02 & 2.15 \\
Upper 95\% Mean & 84.12 & 88.02 & 84.22 \\
Lower 95\% Mean & 71.62 & 75.70 & 75.64 \\
Sample size & 31 & 33 & 64 \\
Sample variance & 290.12 & 301.88 & 295.53 \\
Skewness (g1) & -0.90 & 0.65 & -0.06 \\
Kurtosis (g2) & 0.56 & 0.37 & 0.68 \\
Coef Variation & 21.87 & 21.22 & 21.51 \\
Number missing & 0 & 0 & 0 \\
Median & 81.2 & 79.2 & 80.2 \\
Mode & 96.7 & 115.7 & 75.8 \\
\hline
\end{tabular}


Table A-141. Sample statistics for Change in Sagittal AGF L, showing Edgewise sample alone $(n=31)$, MARA + Edgewise alone $(n=33)$ and the total sample $(n=$ 64).

\begin{tabular}{lccc}
\hline \multicolumn{1}{c}{ Statistic } & $\begin{array}{c}\text { Edgewise } \\
\text { Alone }\end{array}$ & $\begin{array}{c}\text { MARA+Edge } \\
\text { Alone }\end{array}$ & $\begin{array}{c}\text { Total } \\
\text { Sample }\end{array}$ \\
\hline Mean & 7.93 & -0.56 & 3.55 \\
Standard deviation & 12.49 & 7.42 & 10.98 \\
Standard error of mean & 2.24 & 1.29 & 1.37 \\
Upper 95\% Mean & 12.51 & 2.07 & 6.29 \\
Lower 95\% Mean & 3.34 & -3.19 & 0.81 \\
Sample size & 31 & 33 & 64 \\
Sample variance & 156.00 & 55.02 & 120.49 \\
Skewness (g1) & 0.97 & 0.28 & 1.20 \\
Kurtosis (g2) & 2.70 & 0.23 & 3.46 \\
Coef Variation & 157.59 & $-1,330.28$ & 309.07 \\
Number missing & 0 & 0 & 0 \\
Median & 7.8 & -1.3 & 3.6 \\
Mode & 8.0 & -2.3 & 0.4 \\
& & One-sample t-test & 2.59 \\
& & & 0.0120 \\
\hline
\end{tabular}


Table A-142. Sample statistics for Sagittal ACP Right Initial, showing Edgewise sample alone $(n=31)$, MARA+Edgewise alone $(n=33)$ and the total sample $(n=$ 64).

\begin{tabular}{lccc}
\hline \multicolumn{1}{c}{ Statistic } & $\begin{array}{c}\text { Edgewise } \\
\text { Alone }\end{array}$ & $\begin{array}{c}\text { MARA+Edge } \\
\text { Alone }\end{array}$ & $\begin{array}{c}\text { Total } \\
\text { Sample }\end{array}$ \\
\hline Mean & 49.29 & 42.02 & 45.54 \\
Standard deviation & 9.19 & 9.01 & 9.74 \\
Standard error of mean & 1.65 & 1.57 & 1.22 \\
Upper 95\% Mean & 52.66 & 45.21 & 47.97 \\
Lower 95\% Mean & 45.92 & 38.82 & 43.11 \\
Sample size & 31 & 33 & 64 \\
Sample variance & 84.38 & 81.24 & 94.85 \\
Skewness (g1) & 0.18 & 0.78 & 0.40 \\
Kurtosis (g2) & 0.62 & -0.09 & -0.26 \\
Coef Variation & 18.64 & 21.45 & 21.39 \\
Number missing & 0 & 0 & 0 \\
Median & 49.4 & 39.7 & 44.5 \\
Mode &. & 39.7 & 39.7 \\
\hline
\end{tabular}


Table A-143. Sample statistics for Sagittal ACP Right Final, showing Edgewise sample alone $(n=31)$, MARA + Edgewise alone $(n=33)$ and the total sample $(n=$ 64).

\begin{tabular}{lccc}
\hline \multicolumn{1}{c}{ Statistic } & $\begin{array}{c}\text { Edgewise } \\
\text { Alone }\end{array}$ & $\begin{array}{c}\text { MARA+Edge } \\
\text { Alone }\end{array}$ & $\begin{array}{c}\text { Total } \\
\text { Sample }\end{array}$ \\
\hline Mean & 50.06 & 42.25 & 46.03 \\
Standard deviation & 12.23 & 9.31 & 11.43 \\
Standard error of mean & 2.20 & 1.62 & 1.43 \\
Upper 95\% Mean & 54.54 & 45.55 & 48.89 \\
Lower 95\% Mean & 45.57 & 38.95 & 43.17 \\
Sample size & 31 & 33 & 64 \\
Sample variance & 149.63 & 86.60 & 130.72 \\
Skewness (g1) & 0.55 & 0.46 & 0.69 \\
Kurtosis (g2) & -0.31 & -0.33 & 0.15 \\
Coef Variation & 24.44 & 22.03 & 24.84 \\
Number missing & 0 & 0 & 0 \\
Median & 48.1 & 40.5 & 44.1 \\
Mode &. & 35.6 & 35.6 \\
\hline
\end{tabular}


Table A-144. Sample statistics for Change in Sagittal ACP R, showing Edgewise sample alone $(n=31)$, MARA + Edgewise alone $(n=33)$ and the total sample $(n=$ 64).

\begin{tabular}{|c|c|c|c|}
\hline Statistic & $\begin{array}{c}\text { Edgewise } \\
\text { Alone } \\
\end{array}$ & $\begin{array}{c}\text { MARA+Edge } \\
\text { Alone }\end{array}$ & $\begin{array}{c}\text { Total } \\
\text { Sample }\end{array}$ \\
\hline Mean & 0.77 & 0.23 & 0.49 \\
\hline Standard deviation & 8.33 & 7.04 & 7.63 \\
\hline Standard error of mean & 1.50 & 1.22 & 0.95 \\
\hline Upper 95\% Mean & 3.83 & 2.72 & 2.40 \\
\hline Lower 95\% Mean & -2.28 & -2.27 & -1.42 \\
\hline Sample size & 31 & 33 & 64 \\
\hline Sample variance & 69.37 & 49.52 & 58.26 \\
\hline Skewness (g1) & 0.70 & 0.27 & 0.54 \\
\hline Kurtosis (g2) & 1.10 & 0.54 & 0.89 \\
\hline Coef Variation & $1,080.33$ & $3,096.19$ & $1,555.74$ \\
\hline Number missing & 0 & 0 & 0 \\
\hline Median & 0.0 & 0.9 & 0.4 \\
\hline \multirow[t]{3}{*}{ Mode } & -8.8 & . & -5.2 \\
\hline & & One-sample t-test & 0.51 \\
\hline & & P-value (two tail) & 0.6089 \\
\hline
\end{tabular}


Table A-145. Sample statistics for Sagittal AAE Right Initial, showing Edgewise sample alone $(n=31)$, MARA+Edgewise alone $(n=33)$ and the total sample $(n=$ 64).

\begin{tabular}{lccc}
\hline \multicolumn{1}{c}{ Statistic } & $\begin{array}{c}\text { Edgewise } \\
\text { Alone }\end{array}$ & $\begin{array}{c}\text { MARA+Edge } \\
\text { Alone }\end{array}$ & $\begin{array}{c}\text { Total } \\
\text { Sample }\end{array}$ \\
\hline Mean & 86.49 & 96.43 & 91.62 \\
Standard deviation & 21.03 & 23.52 & 22.73 \\
Standard error of mean & 3.78 & 4.09 & 2.84 \\
Upper 95\% Mean & 94.20 & 104.77 & 97.29 \\
Lower 95\% Mean & 78.77 & 88.09 & 85.94 \\
Sample size & 31 & 33 & 64 \\
Sample variance & 442.19 & 553.17 & 516.64 \\
Skewness (g1) & 0.14 & 0.04 & 0.15 \\
Kurtosis (g2) & -0.88 & 0.40 & -0.14 \\
Coef Variation & 24.31 & 24.39 & 24.81 \\
Number missing & 0 & 0 & 0 \\
Median & 84.6 & 97.1 & 92.8 \\
Mode & 49.7 & 68.8 & 94.2 \\
\hline
\end{tabular}


Table A-146. Sample statistics for Sagittal AAE Right Final, showing Edgewise sample alone $(n=31)$, MARA+Edgewise alone $(n=33)$ and the total sample $(n=$ 64).

\begin{tabular}{lccc}
\hline \multicolumn{1}{c}{ Statistic } & $\begin{array}{c}\text { Edgewise } \\
\text { Alone }\end{array}$ & $\begin{array}{c}\text { MARA+Edge } \\
\text { Alone }\end{array}$ & $\begin{array}{c}\text { Total } \\
\text { Sample }\end{array}$ \\
\hline Mean & 97.32 & 102.21 & 99.84 \\
Standard deviation & 23.29 & 25.81 & 24.55 \\
Standard error of mean & 4.18 & 4.49 & 3.07 \\
Upper 95\% Mean & 105.87 & 111.36 & 105.97 \\
Lower 95\% Mean & 88.78 & 93.06 & 93.71 \\
Sample size & 31 & 33 & 64 \\
Sample variance & 542.53 & 666.01 & 602.69 \\
Skewness (g1) & 0.27 & 0.32 & 0.32 \\
Kurtosis (g2) & -0.85 & -0.11 & -0.39 \\
Coef Variation & 23.93 & 25.25 & 24.59 \\
Number missing & 0 & 0 & 0 \\
Median & 103.1 & 98.8 & 99.2 \\
Mode & 103.1 & 120.6 & 70.9 \\
\hline
\end{tabular}


Table A-147. Sample statistics for Change in Sagittal AAE R, showing Edgewise sample alone $(n=31)$, MARA + Edgewise alone $(n=33)$ and the total sample $(n=$ 64).

\begin{tabular}{|c|c|c|c|}
\hline Statistic & $\begin{array}{c}\text { Edgewise } \\
\text { Alone } \\
\end{array}$ & $\begin{array}{c}\text { MARA+Edge } \\
\text { Alone }\end{array}$ & $\begin{array}{c}\text { Total } \\
\text { Sample }\end{array}$ \\
\hline Mean & 10.84 & 5.77 & 8.23 \\
\hline Standard deviation & 16.63 & 14.37 & 15.59 \\
\hline Standard error of mean & 2.99 & 2.50 & 1.95 \\
\hline Upper 95\% Mean & 16.94 & 10.87 & 12.12 \\
\hline Lower 95\% Mean & 4.74 & 0.68 & 4.33 \\
\hline Sample size & 31 & 33 & 64 \\
\hline Sample variance & 276.58 & 206.45 & 243.07 \\
\hline Skewness (g1) & 0.53 & 0.67 & 0.62 \\
\hline Kurtosis (g2) & 0.59 & 0.69 & 0.57 \\
\hline Coef Variation & 153.48 & 248.90 & 189.55 \\
\hline Number missing & 0 & 0 & 0 \\
\hline Median & 9.5 & 3.1 & 6.3 \\
\hline \multirow[t]{3}{*}{ Mode } & 6.1 & . & 9.5 \\
\hline & & One-sample t-test & 4.22 \\
\hline & & P-value (two tail) & $<0.0001$ \\
\hline
\end{tabular}


Table A-148. Sample statistics for Sagittal AGF Right Initial, showing Edgewise sample alone $(n=31)$, MARA+Edgewise alone $(n=33)$ and the total sample $(n=$ 64).

\begin{tabular}{lccc}
\hline \multicolumn{1}{c}{ Statistic } & $\begin{array}{c}\text { Edgewise } \\
\text { Alone }\end{array}$ & $\begin{array}{c}\text { MARA+Edge } \\
\text { Alone }\end{array}$ & $\begin{array}{c}\text { Total } \\
\text { Sample }\end{array}$ \\
\hline Mean & 78.26 & 82.48 & 80.44 \\
Standard deviation & 15.31 & 15.74 & 15.56 \\
Standard error of mean & 2.75 & 2.74 & 1.94 \\
Upper 95\% Mean & 83.88 & 88.06 & 84.32 \\
Lower 95\% Mean & 72.65 & 76.90 & 76.55 \\
Sample size & 31 & 33 & 64 \\
Sample variance & 234.28 & 247.90 & 242.00 \\
Skewness (g1) & -0.27 & 0.97 & 0.39 \\
Kurtosis (g2) & 0.44 & 1.84 & 1.33 \\
Coef Variation & 19.56 & 19.09 & 19.34 \\
Number missing & 0 & 0 & 0 \\
Median & 79.6 & 78.8 & 79.1 \\
Mode &. & 69.2 & 69.2 \\
\hline
\end{tabular}


Table A-149. Sample statistics for Sagittal AGF Right Final, showing Edgewise sample alone $(n=31)$, MARA+Edgewise alone $(n=33)$ and the total sample $(n=$ 64).

\begin{tabular}{lccc}
\hline \multicolumn{1}{c}{ Statistic } & $\begin{array}{c}\text { Edgewise } \\
\text { Alone }\end{array}$ & $\begin{array}{c}\text { MARA+Edge } \\
\text { Alone }\end{array}$ & $\begin{array}{c}\text { Total } \\
\text { Sample }\end{array}$ \\
\hline Mean & 84.98 & 84.04 & \\
Standard deviation & 14.11 & 16.28 & 15.16 \\
Standard error of mean & 2.54 & 2.83 & 1.89 \\
Upper 95\% Mean & 90.15 & 89.81 & 88.28 \\
Lower 95\% Mean & 79.80 & 78.26 & 80.71 \\
Sample size & 31 & 33 & 64 \\
Sample variance & 199.22 & 265.11 & 229.75 \\
Skewness (g1) & -0.22 & 0.47 & 0.19 \\
Kurtosis (g2) & -0.57 & 1.84 & 0.87 \\
Coef Variation & 16.61 & 19.38 & 17.94 \\
Number missing & 0 & 0 & 0 \\
Median & 84.8 & 82.7 & 83.8 \\
Mode &. &. &. \\
\hline
\end{tabular}


Table A-150. Sample statistics for Change in Sagittal AGF R, showing Edgewise sample alone $(n=31)$, MARA + Edgewise alone $(n=33)$ and the total sample $(n=$ 64).

\begin{tabular}{|c|c|c|c|}
\hline Statistic & $\begin{array}{c}\text { Edgewise } \\
\text { Alone }\end{array}$ & $\begin{array}{c}\text { MARA+Edge } \\
\text { Alone }\end{array}$ & $\begin{array}{c}\text { Total } \\
\text { Sample }\end{array}$ \\
\hline Mean & 6.71 & 1.55 & 4.05 \\
\hline Standard deviation & 13.00 & 7.97 & 10.93 \\
\hline Standard error of mean & 2.33 & 1.39 & 1.37 \\
\hline Upper 95\% Mean & 11.48 & 4.38 & 6.78 \\
\hline Lower 95\% Mean & 1.95 & -1.27 & 1.32 \\
\hline Sample size & 31 & 33 & 64 \\
\hline Sample variance & 168.90 & 63.52 & 119.45 \\
\hline Skewness (g1) & 0.40 & 0.34 & 0.69 \\
\hline Kurtosis (g2) & -0.03 & -0.23 & 0.70 \\
\hline Coef Variation & 193.60 & 512.69 & 269.65 \\
\hline Number missing & 0 & 0 & 0 \\
\hline Median & 6.0 & 0.7 & 3.1 \\
\hline \multirow[t]{3}{*}{ Mode } & . & -0.7 & -0.7 \\
\hline & & One-sample t-test & 2.97 \\
\hline & & P-value (two tail) & 0.0042 \\
\hline
\end{tabular}


Table A-151. Sample statistics for Coronal MJS Left Initial, showing Edgewise sample alone $(n=31)$, MARA+Edgewise alone $(n=33)$ and the total sample $(n=$ 64).

\begin{tabular}{lccc}
\hline \multicolumn{1}{c}{ Statistic } & $\begin{array}{c}\text { Edgewise } \\
\text { Alone }\end{array}$ & $\begin{array}{c}\text { MARA+Edge } \\
\text { Alone }\end{array}$ & $\begin{array}{c}\text { Total } \\
\text { Sample }\end{array}$ \\
\hline Mean & 1.86 & 1.87 & 1.87 \\
Standard deviation & 0.64 & 0.74 & 0.68 \\
Standard error of mean & 0.11 & 0.13 & 0.09 \\
Upper 95\% Mean & 2.09 & 2.13 & 2.04 \\
Lower 95\% Mean & 1.62 & 1.61 & 1.69 \\
Sample size & 31 & 33 & 64 \\
Sample variance & 0.40 & 0.54 & 0.47 \\
Skewness (g1) & 0.40 & 0.71 & 0.59 \\
Kurtosis (g2) & -0.25 & 0.41 & 0.15 \\
Coef Variation & 34.20 & 39.31 & 36.66 \\
Number missing & 0 & 0 & 0 \\
Median & 1.9 & 1.8 & 1.9 \\
Mode & 1.1 & 1.3 & 1.1 \\
\hline
\end{tabular}


Table A-152. Sample statistics for Coronal MJS Left Final, showing Edgewise sample alone $(n=31)$, MARA + Edgewise alone $(n=33)$ and the total sample $(n=$ 64).

\begin{tabular}{lccc}
\hline \multicolumn{1}{c}{ Statistic } & $\begin{array}{c}\text { Edgewise } \\
\text { Alone }\end{array}$ & $\begin{array}{c}\text { MARA+Edge } \\
\text { Alone }\end{array}$ & $\begin{array}{c}\text { Total } \\
\text { Sample }\end{array}$ \\
\hline Mean & 1.80 & 2.16 & 1.99 \\
Standard deviation & 0.60 & 1.08 & 0.89 \\
Standard error of mean & 0.11 & 0.19 & 0.11 \\
Upper 95\% Mean & 2.02 & 2.55 & 2.21 \\
Lower 95\% Mean & 1.58 & 1.78 & 1.77 \\
Sample size & 31 & 33 & 64 \\
Sample variance & 0.36 & 1.17 & 0.80 \\
Skewness (g1) & -0.49 & 1.06 & 1.16 \\
Kurtosis (g2) & 0.29 & 0.93 & 2.44 \\
Coef Variation & 33.38 & 49.95 & 44.93 \\
Number missing & 0 & 0 & 0 \\
Median & 1.8 & 2.0 & 1.9 \\
Mode & 1.6 & 1.7 & 1.6 \\
\hline
\end{tabular}


Table A-153. Sample statistics for Change in Coronal MJS L, showing Edgewise sample alone $(n=31)$, MARA + Edgewise alone $(n=33)$ and the total sample $(n=$ 64).

\begin{tabular}{lccc}
\hline \multicolumn{1}{c}{ Statistic } & $\begin{array}{c}\text { Edgewise } \\
\text { Alone }\end{array}$ & $\begin{array}{c}\text { MARA+Edge } \\
\text { Alone }\end{array}$ & $\begin{array}{c}\text { Total } \\
\text { Sample }\end{array}$ \\
\hline Mean & -0.05 & 0.29 & 0.12 \\
Standard deviation & 0.62 & 1.06 & 0.89 \\
Standard error of mean & 0.11 & 0.19 & 0.11 \\
Upper 95\% Mean & 0.17 & 0.67 & 0.35 \\
Lower 95\% Mean & -0.28 & -0.09 & -0.10 \\
Sample size & 31 & 33 & 64 \\
Sample variance & 0.38 & 1.13 & 0.79 \\
Skewness (g1) & -0.52 & 1.35 & 1.34 \\
Kurtosis (g2) & 4.16 & 2.83 & 4.69 \\
Coef Variation & $-1,129.84$ & 365.45 & 719 \\
Number missing & 0 & 0 & 0 \\
Median & -0.1 & 0.2 & 0.0 \\
Mode & -0.3 & -0.4 & -0.4 \\
& & P-value (two tail) & 0.2699 \\
\hline & & &
\end{tabular}


Table A-154. Sample statistics for Coronal SJS Left Initial, showing Edgewise sample alone $(n=31)$, MARA+Edgewise alone $(n=33)$ and the total sample $(n=$ 64).

\begin{tabular}{lccc}
\hline \multicolumn{1}{c}{ Statistic } & $\begin{array}{c}\text { Edgewise } \\
\text { Alone }\end{array}$ & $\begin{array}{c}\text { MARA+Edge } \\
\text { Alone }\end{array}$ & $\begin{array}{c}\text { Total } \\
\text { Sample }\end{array}$ \\
\hline Mean & 1.98 & 1.78 & 1.87 \\
Standard deviation & 0.83 & 0.61 & 0.72 \\
Standard error of mean & 0.15 & 0.11 & 0.09 \\
Upper 95\% Mean & 2.28 & 1.99 & 2.05 \\
Lower 95\% Mean & 1.67 & 1.56 & 1.69 \\
Sample size & 31 & 33 & 64 \\
Sample variance & 0.68 & 0.37 & 0.52 \\
Skewness (g1) & 0.97 & -0.36 & 0.68 \\
Kurtosis (g2) & 0.68 & 0.23 & 1.18 \\
Coef Variation & 41.84 & 34.31 & 38.67 \\
Number missing & 0 & 0 & 0 \\
Median & 1.6 & 1.8 & 1.8 \\
Mode & 1.4 & 1.4 & 1.4 \\
\hline
\end{tabular}


Table A-155. Sample statistics for Coronal SJS Left Final, showing Edgewise sample alone $(n=31)$, MARA+Edgewise alone $(n=33)$ and the total sample $(n=$ 64).

\begin{tabular}{lccc}
\hline \multicolumn{1}{c}{ Statistic } & $\begin{array}{c}\text { Edgewise } \\
\text { Alone }\end{array}$ & $\begin{array}{c}\text { MARA+Edge } \\
\text { Alone }\end{array}$ & $\begin{array}{c}\text { Total } \\
\text { Sample }\end{array}$ \\
\hline Mean & 1.97 & 1.83 & 1.90 \\
Standard deviation & 0.69 & 0.79 & 0.74 \\
Standard error of mean & 0.12 & 0.14 & 0.09 \\
Upper 95\% Mean & 2.23 & 2.11 & 2.09 \\
Lower 95\% Mean & 1.72 & 1.55 & 1.72 \\
Sample size & 31 & 33 & 64 \\
Sample variance & 0.47 & 0.63 & 0.55 \\
Skewness (g1) & 0.22 & 0.44 & 0.31 \\
Kurtosis (g2) & -0.24 & 0.15 & -0.08 \\
Coef Variation & 34.82 & 43.31 & 39.01 \\
Number missing & 0 & 0 & 0 \\
Median & 2.0 & 1.9 & 1.9 \\
Mode & 1.1 & 1.9 & 1.9 \\
\hline
\end{tabular}


Table A-156. Sample statistics for Change in Coronal SJS L, showing Edgewise sample alone $(n=31)$, MARA+Edgewise alone $(n=33)$ and the total sample $(n=$ 64).

\begin{tabular}{lccc}
\hline \multicolumn{1}{c}{ Statistic } & $\begin{array}{c}\text { Edgewise } \\
\text { Alone }\end{array}$ & $\begin{array}{c}\text { MARA+Edge } \\
\text { Alone }\end{array}$ & $\begin{array}{c}\text { Total } \\
\text { Sample }\end{array}$ \\
Mean & 0.00 & 0.06 & 0.03 \\
Standard deviation & 0.72 & 0.89 & 0.81 \\
Standard error of mean & 0.13 & 0.16 & 0.10 \\
Upper 95\% Mean & 0.26 & 0.37 & 0.23 \\
Lower 95\% Mean & -0.27 & -0.26 & -0.17 \\
Sample size & 31 & 33 & 64 \\
Sample variance & 0.52 & 0.80 & 0.65 \\
Skewness (g1) & -0.68 & 0.45 & 0.10 \\
Kurtosis (g2) & 0.56 & 1.80 & 1.52 \\
Coef Variation & $-22,303.98$ & $1,549.85$ & $2,870.76$ \\
Number missing & 0 & 0 & 0 \\
Median & 0.1 & 0.1 & 0.1 \\
Mode & 0.0 & -0.2 & 0.0 \\
& & One-sample t-test & 0.28 \\
& & & 0.7814 \\
\hline
\end{tabular}


Table A-157. Sample statistics for Coronal LJS Left Initial, showing Edgewise sample alone $(n=31)$, MARA + Edgewise alone $(n=33)$ and the total sample $(n=$ 64).

\begin{tabular}{lccc}
\hline \multicolumn{1}{c}{ Statistic } & $\begin{array}{c}\text { Edgewise } \\
\text { Alone }\end{array}$ & $\begin{array}{c}\text { MARA+Edge } \\
\text { Alone }\end{array}$ & $\begin{array}{c}\text { Total } \\
\text { Sample }\end{array}$ \\
\hline Mean & 2.65 & 2.30 & 2.47 \\
Standard deviation & 0.65 & 0.79 & 0.74 \\
Standard error of mean & 0.12 & 0.14 & 0.09 \\
Upper 95\% Mean & 2.89 & 2.58 & 2.66 \\
Lower 95\% Mean & 2.42 & 2.02 & 2.28 \\
Sample size & 31 & 33 & 64 \\
Sample variance & 0.43 & 0.63 & 0.55 \\
Skewness (g1) & -0.44 & 0.72 & 0.16 \\
Kurtosis (g2) & 1.56 & 1.41 & 0.68 \\
Coef Variation & 24.56 & 34.48 & 30.12 \\
Number missing & 0 & 0 & 0 \\
Median & 2.7 & 2.4 & 2.5 \\
Mode & 3.0 & 2.4 & 2.5 \\
\hline
\end{tabular}


Table A-158. Sample statistics for Coronal LJS Left Final, showing Edgewise sample alone $(n=31)$, MARA + Edgewise alone $(n=33)$ and the total sample $(n=$ 64).

\begin{tabular}{lccc}
\hline \multicolumn{1}{c}{ Statistic } & $\begin{array}{c}\text { Edgewise } \\
\text { Alone }\end{array}$ & $\begin{array}{c}\text { MARA+Edge } \\
\text { Alone }\end{array}$ & $\begin{array}{c}\text { Total } \\
\text { Sample }\end{array}$ \\
\hline Mean & 2.53 & 2.37 & 2.45 \\
Standard deviation & 0.70 & 0.72 & 0.71 \\
Standard error of mean & 0.13 & 0.13 & 0.09 \\
Upper 95\% Mean & 2.79 & 2.62 & 2.62 \\
Lower 95\% Mean & 2.27 & 2.11 & 2.27 \\
Sample size & 31 & 33 & 64 \\
Sample variance & 0.49 & 0.53 & 0.51 \\
Skewness (g1) & 0.51 & -0.13 & 0.15 \\
Kurtosis (g2) & -0.89 & -0.56 & -0.58 \\
Coef Variation & 27.81 & 30.63 & 29.18 \\
Number missing & 0 & 0 & 0 \\
Median & 2.4 & 2.5 & 2.5 \\
Mode & 1.9 & 1.4 & 2.5 \\
\hline
\end{tabular}


Table A-159. Sample statistics for Change in Coronal LJS L, showing Edgewise sample alone $(n=31)$, MARA+Edgewise alone $(n=33)$ and the total sample $(n=$ 64).

\begin{tabular}{lccc}
\hline \multicolumn{1}{c}{ Statistic } & $\begin{array}{c}\text { Edgewise } \\
\text { Alone }\end{array}$ & $\begin{array}{c}\text { MARA+Edge } \\
\text { Alone }\end{array}$ & $\begin{array}{c}\text { Total } \\
\text { Sample }\end{array}$ \\
\hline Mean & -0.13 & 0.07 & -0.03 \\
Standard deviation & 0.69 & 0.65 & 0.67 \\
Standard error of mean & 0.12 & 0.11 & 0.08 \\
Upper 95\% Mean & 0.13 & 0.30 & 0.14 \\
Lower 95\% Mean & -0.38 & -0.16 & -0.19 \\
Sample size & 31 & 33 & 64 \\
Sample variance & 0.48 & 0.43 & 0.45 \\
Skewness (g1) & 0.41 & -0.03 & 0.16 \\
Kurtosis (g2) & 0.20 & -0.47 & -0.31 \\
Coef Variation & -549.54 & 937.76 & $-2,696.03$ \\
Number missing & 0 & 0 & 0 \\
Median & -0.1 & 0.1 & -0.1 \\
Mode & -0.1 & 0.1 & -0.1 \\
& & P-value (two tail) & 0.7676 \\
\hline
\end{tabular}


Table A-160. Sample statistics for Coronal MJS Right Initial, showing Edgewise sample alone $(n=31)$, MARA + Edgewise alone $(n=33)$ and the total sample $(n=$ 64).

\begin{tabular}{lccc}
\hline \multicolumn{1}{c}{ Statistic } & $\begin{array}{c}\text { Edgewise } \\
\text { Alone }\end{array}$ & $\begin{array}{c}\text { MARA+Edge } \\
\text { Alone }\end{array}$ & $\begin{array}{c}\text { Total } \\
\text { Sample }\end{array}$ \\
\hline Mean & 1.76 & 1.82 & 1.79 \\
Standard deviation & 0.80 & 0.83 & 0.81 \\
Standard error of mean & 0.14 & 0.14 & 0.10 \\
Upper 95\% Mean & 2.06 & 2.11 & 2.00 \\
Lower 95\% Mean & 1.47 & 1.53 & 1.59 \\
Sample size & 31 & 33 & 64 \\
Sample variance & 0.65 & 0.68 & 0.66 \\
Skewness (g1) & 0.56 & 0.69 & 0.62 \\
Kurtosis (g2) & -0.50 & -0.07 & -0.33 \\
Coef Variation & 45.56 & 45.36 & 45.13 \\
Number missing & 0 & 0 & 0 \\
Median & 1.7 & 1.8 & 1.8 \\
Mode & 0.7 & 2.0 & 1.4 \\
\hline
\end{tabular}


Table A-161. Sample statistics for Coronal MJS Right Final, showing Edgewise sample alone $(n=31)$, MARA+Edgewise alone $(n=33)$ and the total sample $(n=$ 64).

\begin{tabular}{lccc}
\hline \multicolumn{1}{c}{ Statistic } & $\begin{array}{c}\text { Edgewise } \\
\text { Alone }\end{array}$ & $\begin{array}{c}\text { MARA+Edge } \\
\text { Alone }\end{array}$ & $\begin{array}{c}\text { Total } \\
\text { Sample }\end{array}$ \\
\hline Mean & 1.93 & 1.63 & 1.78 \\
Standard deviation & 0.69 & 0.81 & 0.76 \\
Standard error of mean & 0.12 & 0.14 & 0.10 \\
Upper 95\% Mean & 2.19 & 1.92 & 1.97 \\
Lower 95\% Mean & 1.68 & 1.35 & 1.59 \\
Sample size & 31 & 33 & 64 \\
Sample variance & 0.48 & 0.65 & 0.58 \\
Skewness (g1) & 0.88 & 0.78 & 0.67 \\
Kurtosis (g2) & 0.40 & 0.19 & 0.09 \\
Coef Variation & 35.91 & 49.47 & 42.96 \\
Number missing & 0 & 0 & 0 \\
Median & 1.9 & 1.5 & 1.7 \\
Mode & 1.4 & 0.7 & 0.7 \\
\hline
\end{tabular}


Table A-162. Sample statistics for Change in Coronal MJS R, showing Edgewise sample alone $(n=31)$, MARA + Edgewise alone $(n=33)$ and the total sample $(n=$ 64).

\begin{tabular}{lccc}
\hline \multicolumn{1}{c}{ Statistic } & $\begin{array}{c}\text { Edgewise } \\
\text { Alone }\end{array}$ & $\begin{array}{c}\text { MARA+Edge } \\
\text { Alone }\end{array}$ & $\begin{array}{c}\text { Total } \\
\text { Sample }\end{array}$ \\
\hline Mean & 0.17 & -0.19 & -0.02 \\
Standard deviation & 0.61 & 0.79 & 0.73 \\
Standard error of mean & 0.11 & 0.14 & 0.09 \\
Upper 95\% Mean & 0.39 & 0.09 & 0.17 \\
Lower 95\% Mean & -0.05 & -0.47 & -0.20 \\
Sample size & 31 & 33 & 64 \\
Sample variance & 0.37 & 0.63 & 0.53 \\
Skewness (g1) & 0.72 & -0.39 & -0.25 \\
Kurtosis (g2) & 0.72 & 2.05 & 2.07 \\
Coef Variation & 360.79 & -422.21 & $-4,642.10$ \\
Number missing & 0 & 0 & 0 \\
Median & 0.1 & -0.1 & 0.0 \\
Mode & 0.3 & 0.0 & 0.0 \\
& & One-sample t-test & 0.17 \\
& & & 0.8637 \\
\hline
\end{tabular}


Table A-163. Sample statistics for Coronal SJS Right Initial, showing Edgewise sample alone $(n=31)$, MARA+Edgewise alone $(n=33)$ and the total sample $(n=$ 64).

\begin{tabular}{lccc}
\hline \multicolumn{1}{c}{ Statistic } & $\begin{array}{c}\text { Edgewise } \\
\text { Alone }\end{array}$ & $\begin{array}{c}\text { MARA+Edge } \\
\text { Alone }\end{array}$ & $\begin{array}{c}\text { Total } \\
\text { Sample }\end{array}$ \\
\hline Mean & 1.85 & 1.95 & 1.90 \\
Standard deviation & 0.57 & 0.58 & 0.57 \\
Standard error of mean & 0.10 & 0.10 & 0.07 \\
Upper 95\% Mean & 2.06 & 2.15 & 2.04 \\
Lower 95\% Mean & 1.65 & 1.74 & 1.76 \\
Sample size & 31 & 33 & 64 \\
Sample variance & 0.33 & 0.34 & 0.33 \\
Skewness (g1) & 0.20 & -0.56 & -0.19 \\
Kurtosis (g2) & -0.92 & -0.22 & -0.71 \\
Coef Variation & 30.78 & 29.92 & 30.18 \\
Number missing & 0 & 0 & 0 \\
Median & 1.9 & 2.1 & 2.0 \\
Mode & 1.1 & 2.1 & 2.3 \\
\hline
\end{tabular}


Table A-164. Sample statistics for Coronal SJS Right Final, showing Edgewise sample alone $(n=31)$, MARA+Edgewise alone $(n=33)$ and the total sample $(n=$ 64).

\begin{tabular}{lccc}
\hline \multicolumn{1}{c}{ Statistic } & $\begin{array}{c}\text { Edgewise } \\
\text { Alone }\end{array}$ & $\begin{array}{c}\text { MARA+Edge } \\
\text { Alone }\end{array}$ & $\begin{array}{c}\text { Total } \\
\text { Sample }\end{array}$ \\
\hline Mean & 1.92 & 1.68 & 1.80 \\
Standard deviation & 0.61 & 0.79 & 0.71 \\
Standard error of mean & 0.11 & 0.14 & 0.09 \\
Upper 95\% Mean & 2.14 & 1.97 & 1.98 \\
Lower 95\% Mean & 1.70 & 1.40 & 1.62 \\
Sample size & 31 & 33 & 64 \\
Sample variance & 0.37 & 0.63 & 0.51 \\
Skewness (g1) & 0.05 & 1.99 & 1.24 \\
Kurtosis (g2) & -0.82 & 6.33 & 3.49 \\
Coef Variation & 31.52 & 47.07 & 39.62 \\
Number missing & 0 & 0 & 0 \\
Median & 2.1 & 1.5 & 1.7 \\
Mode & 2.3 & 1.5 & 1.4 \\
\hline
\end{tabular}


Table A-165. Sample statistics for Change in Coronal SJS R, showing Edgewise sample alone $(n=31)$, MARA+Edgewise alone $(n=33)$ and the total sample $(n=$ 64).

\begin{tabular}{lccc}
\hline \multicolumn{1}{c}{ Statistic } & $\begin{array}{c}\text { Edgewise } \\
\text { Alone }\end{array}$ & $\begin{array}{c}\text { MARA+Edge } \\
\text { Alone }\end{array}$ & $\begin{array}{c}\text { Total } \\
\text { Sample }\end{array}$ \\
\hline Mean & 0.07 & -0.26 & -0.10 \\
Standard deviation & 0.65 & 0.93 & 0.82 \\
Standard error of mean & 0.12 & 0.16 & 0.10 \\
Upper 95\% Mean & 0.31 & 0.07 & 0.10 \\
Lower 95\% Mean & -0.17 & -0.59 & -0.31 \\
Sample size & 31 & 33 & 64 \\
Sample variance & 0.43 & 0.86 & 0.67 \\
Skewness (g1) & 0.89 & 1.89 & 1.37 \\
Kurtosis (g2) & 0.91 & 6.45 & 4.40 \\
Coef Variation & 964.54 & -355.71 & -804.22 \\
Number missing & 0 & 0 & 0 \\
Median & -0.1 & -0.4 & -0.2 \\
Mode & -0.4 & -0.9 & -0.4 \\
& & One-sample t-test & -0.99 \\
& & & 0.3237 \\
\hline
\end{tabular}


Table A-166. Sample statistics for Coronal LJS Right Initial, showing Edgewise sample alone $(n=31)$, MARA+Edgewise alone $(n=33)$ and the total sample $(n=$ 64).

\begin{tabular}{lccc}
\hline \multicolumn{1}{c}{ Statistic } & $\begin{array}{c}\text { Edgewise } \\
\text { Alone }\end{array}$ & $\begin{array}{c}\text { MARA+Edge } \\
\text { Alone }\end{array}$ & $\begin{array}{c}\text { Total } \\
\text { Sample }\end{array}$ \\
\hline Mean & 2.47 & 2.26 & 2.36 \\
Standard deviation & 0.61 & 0.60 & 0.61 \\
Standard error of mean & 0.11 & 0.10 & 0.08 \\
Upper 95\% Mean & 2.69 & 2.48 & 2.52 \\
Lower 95\% Mean & 2.25 & 2.05 & 2.21 \\
Sample size & 31 & 33 & 64 \\
Sample variance & 0.37 & 0.36 & 0.37 \\
Skewness (g1) & 0.07 & 0.26 & 0.16 \\
Kurtosis (g2) & -0.24 & -0.17 & -0.32 \\
Coef Variation & 24.53 & 26.41 & 25.64 \\
Number missing & 0 & 0 & 0 \\
Median & 2.4 & 2.1 & 2.3 \\
Mode & 1.9 & 2.1 & 2.1 \\
\hline
\end{tabular}


Table A-167. Sample statistics for Coronal LJS Right Final, showing Edgewise sample alone $(n=31)$, MARA+Edgewise alone $(n=33)$ and the total sample $(n=$ 64).

\begin{tabular}{lccc}
\hline \multicolumn{1}{c}{ Statistic } & $\begin{array}{c}\text { Edgewise } \\
\text { Alone }\end{array}$ & $\begin{array}{c}\text { MARA+Edge } \\
\text { Alone }\end{array}$ & $\begin{array}{c}\text { Total } \\
\text { Sample }\end{array}$ \\
\hline Mean & 2.55 & 2.32 & 2.43 \\
Standard deviation & 0.68 & 0.71 & 0.70 \\
Standard error of mean & 0.12 & 0.12 & 0.09 \\
Upper 95\% Mean & 2.80 & 2.57 & 2.60 \\
Lower 95\% Mean & 2.30 & 2.06 & 2.25 \\
Sample size & 31 & 33 & 64 \\
Sample variance & 0.47 & 0.50 & 0.49 \\
Skewness (g1) & 0.86 & 0.32 & 0.52 \\
Kurtosis (g2) & 0.33 & -0.39 & 0.02 \\
Coef Variation & 26.84 & 30.54 & 28.85 \\
Number missing & 0 & 0 & 0 \\
Median & 2.4 & 2.4 & 2.4 \\
Mode & 2.0 & 2.5 & 2.5 \\
\hline
\end{tabular}


Table A-168. Sample statistics for Change in Coronal LJS R, showing Edgewise sample alone $(n=31)$, MARA+Edgewise alone $(n=33)$ and the total sample $(n=$ 64).

\begin{tabular}{lccc}
\hline \multicolumn{1}{c}{ Statistic } & $\begin{array}{c}\text { Edgewise } \\
\text { Alone }\end{array}$ & $\begin{array}{c}\text { MARA+Edge } \\
\text { Alone }\end{array}$ & $\begin{array}{c}\text { Total } \\
\text { Sample }\end{array}$ \\
\hline Mean & 0.08 & 0.05 & 0.06 \\
Standard deviation & 0.73 & 0.62 & 0.67 \\
Standard error of mean & 0.13 & 0.11 & 0.08 \\
Upper 95\% Mean & 0.35 & 0.27 & 0.23 \\
Lower 95\% Mean & -0.19 & -0.17 & -0.10 \\
Sample size & 31 & 33 & 64 \\
Sample variance & 0.53 & 0.39 & 0.45 \\
Skewness (g1) & 0.52 & 0.41 & 0.48 \\
Kurtosis (g2) & 0.53 & 0.40 & 0.44 \\
Coef Variation & 942.54 & $1,211.41$ & $1,048.93$ \\
Number missing & 0 & 0 & 0 \\
Median & 0.00 & 0.00 & 0.00 \\
Mode & 0.50 & -0.10 & 0.50 \\
& & One-sample t-test & 0.76 \\
& & & 0.4485 \\
\hline
\end{tabular}




\section{APPENDIX B. RESULTS OF ANALYSIS OF VARIANCE FOR TREATMENT OR SEX DIFFERENCES IN THE UNILATERAL (MIDLINE) DIMENSIONS AND TREATMENT, SEX, AND SIDE (LEFT, RIGHT) IN THE BILATERAL DIMENSIONS}

Table B-1. Results of ANOVA testing for treatment (Edgewise with or without MARA) and sex (male, female) differences for SNA at the start of treatment.

\begin{tabular}{lcccc}
\hline \multicolumn{1}{c}{ Source } & df & SSQ & F ratio & P value \\
\hline Treatment & 1 & 0.03 & 0.00 & 0.9618 \\
Sex & 1 & 2.27 & 0.21 & 0.6489 \\
Treatment-by-Sex & 1 & 7.68 & 0.71 & 0.4028 \\
\hline
\end{tabular}

Table B-2. Results of ANOVA testing for treatment (Edgewise with or without MARA) and sex (male, female) differences for SNA at the end of treatment.

\begin{tabular}{llrll}
\hline \multicolumn{1}{c}{ Source } & df & SSQ & F ratio & P value \\
\hline Treatment & 1 & 31.83 & 3.18 & 0.0797 \\
Sex & 1 & 0.01 & 0.00 & 0.9763 \\
Treatment-by-Sex & 1 & 4.22 & 0.42 & 0.5188 \\
\hline
\end{tabular}

Table B-3. Results of ANOVA testing for treatment (Edgewise with or without MARA) and sex (male, female) differences for the treatment change in SNA.

\begin{tabular}{lrrrr}
\hline \multicolumn{1}{c}{ Source } & df & SSQ & F ratio & P value \\
\hline & & & & \\
Treatment & 1 & 30.07 & 18.98 & $<.0001$ \\
Sex & 1 & 1.99 & 1.26 & 0.2666 \\
Treatment-by-Sex & 1 & 0.52 & 0.33 & 0.5704 \\
\hline
\end{tabular}


Table B-4. Results of ANOVA testing for treatment (Edgewise with or without MARA) and sex (male, female) differences for SNB Initial.

\begin{tabular}{lllll}
\hline \multicolumn{1}{c}{ Source } & df & SSQ & F ratio & P value \\
\hline Treatment & 1 & 0.08 & 0.01 & 0.9249 \\
Sex & 1 & 0.16 & 0.02 & 0.8938 \\
Treatment-by-Sex & 1 & 2.33 & 0.26 & 0.6093 \\
\hline
\end{tabular}

Table B-5. Results of ANOVA testing for treatment (Edgewise with or without MARA) and sex (male, female) differences for SNB Final.

\begin{tabular}{lrrrr}
\hline \multicolumn{1}{c}{ Source } & df & SSQ & F ratio & P value \\
\hline & & & & \\
Treatment & 1 & 10.83 & 1.23 & 0.2715 \\
Sex & 1 & 2.38 & 0.27 & 0.6049 \\
Treatment-by-Sex & 1 & 0.86 & 0.10 & 0.7554 \\
\hline
\end{tabular}

Table B-6. Results of ANOVA testing for treatment (Edgewise with or without MARA) and sex (male, female) differences for Change in SNB.

\begin{tabular}{llrll}
\hline \multicolumn{1}{c}{ Source } & df & SSQ & F ratio & P value \\
\hline Treatment & 1 & 12.76 & 9.47 & 0.0031 \\
Sex & 1 & 3.77 & 2.80 & 0.0997 \\
Treatment-by-Sex & 1 & 0.36 & 0.27 & 0.6078 \\
\hline
\end{tabular}


Table B-7. Results of ANOVA testing for treatment (Edgewise with or without MARA) and sex (male, female) differences for ANB Initial.

\begin{tabular}{lcccc}
\hline \multicolumn{1}{c}{ Source } & df & SSQ & F ratio & P value \\
\hline Treatment & 1 & 0.23 & & \\
Sex & 1 & 1.21 & 0.10 & 0.7588 \\
Treatment-by-Sex & 1 & 1.57 & 0.51 & 0.4772 \\
\end{tabular}

Table B-8. Results of ANOVA testing for treatment (Edgewise with or without MARA) and sex (male, female) differences for ANB Final.

\begin{tabular}{lcccc}
\hline \multicolumn{1}{c}{ Source } & df & SSQ & F ratio & P value \\
\hline Treatment & 1 & 5.36 & 1.99 & 0.1631 \\
Sex & 1 & 2.43 & 0.90 & 0.3455 \\
Treatment-by-Sex & 1 & 1.45 & 0.54 & 0.4661 \\
\hline
\end{tabular}

Table B-9. Results of ANOVA testing for treatment (Edgewise with or without MARA) and sex (male, female) differences for Change in ANB.

\begin{tabular}{lllll}
\hline \multicolumn{1}{c}{ Source } & df & SSQ & F ratio & P value \\
\hline Treatment & 1 & 3.39 & 2.60 & 0.1120 \\
Sex & 1 & 0.21 & 0.16 & 0.6891 \\
Treatment-by-Sex & 1 & 0.00 & 0.00 & 0.9660 \\
\hline
\end{tabular}


Table B-10. Results of ANOVA testing for treatment (Edgewise with or without MARA) and sex (male, female) differences for Overbite Initial.

\begin{tabular}{lcccc}
\hline \multicolumn{1}{c}{ Source } & df & SSQ & F ratio & P value \\
\hline Treatment & 1 & 0.74 & 0.20 & 0.6559 \\
Sex & 1 & 0.12 & 0.03 & 0.8572 \\
Treatment-by-Sex & 1 & 0.12 & 0.03 & 0.8558 \\
\hline
\end{tabular}

Table B-11. Results of ANOVA testing for treatment (Edgewise with or without MARA) and sex (male, female) differences for Overbite Final.

\begin{tabular}{lcccc}
\hline \multicolumn{1}{c}{ Source } & df & SSQ & F ratio & P value \\
\hline Treatment & 1 & 0.08 & 0.07 & 0.7887 \\
Sex & 1 & 0.03 & 0.03 & 0.8628 \\
Treatment-by-Sex & 1 & 1.20 & 1.10 & 0.2977 \\
\hline
\end{tabular}

Table B-12. Results of ANOVA testing for treatment (Edgewise with or without MARA) and sex (male, female) differences for Change in Overbite.

\begin{tabular}{lllll}
\hline \multicolumn{1}{c}{ Source } & df & SSQ & F ratio & P value \\
\hline Treatment & 1 & 1.31 & 0.41 & 0.5262 \\
Sex & 1 & 0.28 & 0.09 & 0.7690 \\
Treatment-by-Sex & 1 & 2.09 & 0.65 & 0.4228 \\
\hline
\end{tabular}


Table B-13. Results of ANOVA testing for treatment (Edgewise with or without MARA) and sex (male, female) differences for Overjet Initial.

\begin{tabular}{lllll}
\hline \multicolumn{1}{c}{ Source } & df & SSQ & F ratio & P value \\
\hline Treatment & 1 & 1.62 & & \\
Sex & 1 & 0.00 & 0.44 & 0.5114 \\
Treatment-by-Sex & 1 & 3.90 & 0.00 & 0.9792 \\
\end{tabular}

Table B-14. Results of ANOVA testing for treatment (Edgewise with or without MARA) and sex (male, female) differences for Overjet Final.

\begin{tabular}{lllll}
\hline \multicolumn{1}{c}{ Source } & df & SSQ & F ratio & P value \\
\hline Treatment & 1 & 0.08 & 0.07 & 0.7887 \\
Sex & 1 & 0.03 & 0.03 & 0.8628 \\
Treatment-by-Sex & 1 & 1.20 & 1.10 & 0.2977 \\
\hline
\end{tabular}

Table B-15. Results of ANOVA testing for treatment (Edgewise with or without MARA) and sex (male, female) differences for Change in Overjet.

\begin{tabular}{lllll}
\hline \multicolumn{1}{c}{ Source } & df & SSQ & F ratio & P value \\
\hline Treatment & 1 & 1.31 & 0.41 & 0.5262 \\
Sex & 1 & 0.28 & 0.09 & 0.7690 \\
Treatment-by-Sex & 1 & 2.09 & 0.65 & 0.4228 \\
\hline
\end{tabular}


Table B-16. Results of ANOVA testing for treatment (Edgewise with or without MARA) and sex (male, female) differences for Mesial Molar Relation Initial.

\begin{tabular}{lcccc}
\hline \multicolumn{1}{c}{ Source } & df & SSQ & F ratio & P value \\
\hline Treatment & 1 & 0.86 & 0.55 & 0.4619 \\
Sex & 1 & 4.59 & 2.92 & 0.0928 \\
Treatment-by-Sex & 1 & 0.01 & 0.00 & 0.9528 \\
\hline
\end{tabular}

Table B-17. Results of ANOVA testing for treatment (Edgewise with or without MARA) and sex (male, female) differences for Mesial Molar Relation Final.

\begin{tabular}{lrrrr}
\hline \multicolumn{1}{c}{ Source } & df & SSQ & F ratio & P value \\
\hline Treatment & 1 & 13.12 & 12.25 & 0.0009 \\
Sex & 1 & 3.01 & 2.81 & 0.0987 \\
Treatment-by-Sex & 1 & 0.00 & 0.00 & 0.9471 \\
\hline
\end{tabular}

Table B-18. Results of ANOVA testing for treatment (Edgewise with or without MARA) and sex (male, female) differences for Change in Molar Relation.

\begin{tabular}{lllll}
\hline \multicolumn{1}{c}{ Source } & df & SSQ & F ratio & P value \\
\hline Treatment & 1 & 7.26 & 3.31 & 0.0737 \\
Sex & 1 & 0.16 & 0.08 & 0.7850 \\
Treatment-by-Sex & 1 & 0.00 & 0.00 & 0.9970 \\
\hline
\end{tabular}


Table B-19. Results of ANOVA testing for treatment (Edgewise with or without MARA) and sex (male, female) differences for Sella-Vella-to-M Initial.

\begin{tabular}{llrll}
\hline \multicolumn{1}{c}{ Source } & df & SSQ & F ratio & P value \\
\hline Treatment & 1 & 14.67 & & \\
Sex & 1 & 4.19 & 0.75 & 0.3900 \\
Treatment-by-Sex & 1 & 28.52 & 0.21 & 0.6450 \\
\end{tabular}

Table B-20. Results of ANOVA testing for treatment (Edgewise with or without MARA) and sex (male, female) differences for Sella-Vella-to-M Final.

\begin{tabular}{lrrrr}
\hline \multicolumn{1}{c}{ Source } & df & SSQ & F ratio & P value \\
\hline & 1 & 351.43 & 12.46 & \\
Treatment & 1 & 81.64 & 2.89 & 0.0008 \\
Sex & 1 & 13.08 & 0.46 & 0.0941 \\
Treatment-by-Sex & & & & 0.4985 \\
\hline
\end{tabular}

Table B-21. Results of ANOVA testing for treatment (Edgewise with or without MARA) and sex (male, female) differences for Change in Sella-Vertical-to-M.

\begin{tabular}{lrrrr}
\hline \multicolumn{1}{c}{ Source } & df & SSQ & F ratio & P value \\
\hline & 1 & 222.52 & 25.20 & $<0.0001$ \\
Treatment & 1 & 48.82 & 5.53 & 0.0220 \\
Sex & 1 & 2.97 & 0.34 & 0.5641 \\
Treatment-by-Sex & & & & \\
\hline
\end{tabular}


Table B-22. Results of ANOVA testing for treatment (Edgewise with or without MARA) and sex (male, female) differences for Axial APCD Initial.

\begin{tabular}{lllll}
\hline \multicolumn{1}{c}{ Source } & df & SSQ & F ratio & P value \\
\hline Treatment & & & & \\
Sex & 0.3236 & 0.22 & 1,60 & 0.6436 \\
Treatment-by-Sex & 2.6703 & 1.79 & 1,60 & 0.1866 \\
& 9.0793 & 6.07 & 1,60 & 0.0166 \\
\hline
\end{tabular}

Table B-23. Results of ANOVA testing for treatment (Edgewise with or without MARA) and sex (male, female) differences for Axial APCD Final.

\begin{tabular}{lrrrr}
\hline \multicolumn{1}{c}{ Source } & df & SSQ & F ratio & P value \\
\hline Treatment & 0.0506 & 0.05 & 1,60 & 0.8319 \\
Sex & 4.9615 & 4.46 & 1,60 & 0.0389 \\
Treatment-by-Sex & 10.8948 & 9.79 & 1,60 & 0.0027 \\
\hline
\end{tabular}

Table B-24. Results of ANOVA testing for treatment (Edgewise with or without MARA) and sex (male, female) differences for Change in Axial APCD.

\begin{tabular}{lllll}
\hline \multicolumn{1}{c}{ Source } & df & SSQ & F ratio & P value \\
\hline Treatment & 0.1183 & 0.09 & 1,60 & \\
Sex & 0.3520 & 0.26 & 1,60 & 0.7672 \\
Treatment-by-Sex & 0.0827 & 0.06 & 1,60 & 0.6099 \\
\hline
\end{tabular}


Table B-25. Results of mixed-model ANOVA testing for treatment (Edgewise with or without MARA) sex (male, female), and side (left, right TMJ) differences for Axial ML L Initial and Axial ML R Initial.

\begin{tabular}{lcccc}
\hline \multicolumn{1}{c}{ Source } & SSQ & F ratio & df & P value \\
\hline & Between Subjects & & & \\
All Between & 0.1228 & 2.46 & 3,60 & 0.0717 \\
Treatment & 0.0276 & 1.66 & 1,60 & 0.2030 \\
Sex & 0.0781 & 4.69 & 1,60 & 0.0343 \\
Treatment-by-Sex & 0.0260 & 1.56 & 1,60 & 0.2164 \\
& & & & \\
All Within Interactions & Within Subjects & & & \\
Side & 0.0141 & 0.28 & 3,60 & 0.8375 \\
Side-by-Treatment & 0.0086 & 0.52 & 1,60 & 0.4742 \\
Side-by-Sex & 0.0074 & 0.44 & 1,60 & 0.5082 \\
Side-by-Treatment-by-Sex & 0.0023 & 0.14 & 1,60 & 0.7134 \\
& 0.0053 & 0.32 & 1,60 & 0.5760 \\
\hline
\end{tabular}

Table B-26. Results of mixed-model ANOVA testing for treatment (Edgewise with or without MARA) sex (male, female), and side (left, right TMJ) differences for Axial ML L Final and Axial ML R Final.

\begin{tabular}{lcccc}
\hline \multicolumn{1}{c}{ Source } & SSQ & F ratio & df & P value \\
\hline & Between Subjects & & & \\
All Between & 0.1792 & 3.58 & 3,60 & 0.0188 \\
Treatment & 0.0388 & 2.33 & 1,60 & 0.1325 \\
Sex & 0.1121 & 6.72 & 1,60 & 0.0119 \\
Treatment-by-Sex & 0.0408 & 2.45 & 1,60 & 0.1229 \\
& & & & \\
All Within Interactions & Within Subjects & & & \\
Side & 0.0556 & 1.11 & 3,60 & 0.3518 \\
Side-by-Treatment & 0.0059 & 0.36 & 1,60 & 0.5535 \\
Side-by-Sex & 0.0079 & 0.47 & 1,60 & 0.4947 \\
Side-by-Treatment-by-Sex & 0.0301 & 1.80 & 1,60 & 0.1842 \\
& 0.0234 & 1.40 & 1,60 & 0.2411 \\
\hline
\end{tabular}


Table B-27. Results of mixed-model ANOVA testing for treatment (Edgewise with or without MARA) sex (male, female), and side (left, right TMJ) differences for Change in Axial ML $L$ and Change in Axial ML R.

\begin{tabular}{lcccc}
\hline \multicolumn{1}{c}{ Source } & SSQ & F ratio & df & P value \\
\hline & & & & \\
All Between & Between Subjects & & & \\
Treatment & 0.0605 & 1.21 & 3,60 & 0.3138 \\
Sex & 0.0112 & 0.67 & 1,60 & 0.4159 \\
Treatment-by-Sex & 0.0352 & 2.11 & 1,60 & 0.1513 \\
& 0.0175 & 1.05 & 1,60 & 0.3093 \\
All Within Interactions & Within Subjects & & & \\
Side & 0.1108 & 2.22 & 3,60 & 0.0955 \\
Side-by-Treatment & 0.0001 & 0.00 & 1,60 & 0.9529 \\
Side-by-Sex & 0.0428 & 2.57 & 1,60 & 0.1143 \\
Side-by-Treatment-by-Sex & 0.0729 & 4.38 & 1,60 & 0.0407 \\
& 0.0121 & 0.73 & 1,60 & 0.3974 \\
\hline
\end{tabular}

Table B-28. Results of mixed-model ANOVA testing for treatment (Edgewise with or without MARA) sex (male, female), and side (left, right TMJ) differences for Axial AP L Initial and Axial AP R Initial.

\begin{tabular}{lcccc}
\hline \multicolumn{1}{c}{ Source } & SSQ & F ratio & df & P value \\
\hline & & & & \\
All Between & Between Subjects & & & \\
Treatment & 0.1355 & 2.71 & 3,60 & 0.0529 \\
Sex & 0.0169 & 1.01 & 1,60 & 0.3185 \\
Treatment-by-Sex & 0.0944 & 5.66 & 1,60 & 0.0205 \\
& 0.0105 & 0.63 & 1,60 & 0.4307 \\
All Within Interactions & & & & \\
Side & Within Subjects & & & \\
Side-by-Treatment & 0.0736 & 1.47 & 3,60 & 0.2314 \\
Side-by-Sex & 0.0047 & 0.28 & 1,60 & 0.5955 \\
Side-by-Treatment-by-Sex & 0.0002 & 0.01 & 1,60 & 0.9222 \\
& 0.0124 & 0.74 & 1,60 & 0.3923 \\
& 0.0593 & 3.56 & 1,60 & 0.0641 \\
\hline
\end{tabular}


Table B-29. Results of mixed-model ANOVA testing for treatment (Edgewise with or without MARA) sex (male, female), and side (left, right TMJ) differences for Axial AP L F and Axial AP R Final.

\begin{tabular}{lcccc}
\hline \multicolumn{1}{c}{ Source } & SSQ & F ratio & df & P value \\
\hline & Between Subjects & & & \\
All Between & & & \\
Treatment & 0.1053 & 2.11 & 3,60 & 0.1090 \\
Sex & 0.0013 & 0.08 & 1,60 & 0.7850 \\
Treatment-by-Sex & 0.1010 & 6.06 & 1,60 & 0.0167 \\
& 0.0054 & 0.33 & 1,60 & 0.5698 \\
All Within Interactions & Within Subjects & & & \\
Side & 0.0286 & 0.57 & 3,60 & 0.6357 \\
Side-by-Treatment & 0.0001 & 0.01 & 1,60 & 0.9352 \\
Side-by-Sex & 0.0014 & 0.09 & 1,60 & 0.7711 \\
Side-by-Treatment-by-Sex & 0.0060 & 0.36 & 1,60 & 0.5509 \\
& 0.0207 & 1.24 & 1,60 & 0.2697 \\
\hline
\end{tabular}

Table B-30. Results of mixed-model ANOVA testing for treatment (Edgewise with or without MARA) sex (male, female), and side (left, right TMJ) differences for Change in Axial AP L and Change in Axial AP R.

\begin{tabular}{lcccc}
\hline \multicolumn{1}{c}{ Source } & SSQ & F ratio & df & P value \\
\hline & & & & \\
All Between & Between Subjects & & & \\
Treatment & 0.0731 & 1.46 & 3,60 & 0.2342 \\
Sex & 0.0708 & 4.25 & 1,60 & 0.0437 \\
Treatment-by-Sex & 0.0008 & 0.05 & 1,60 & 0.8304 \\
& 0.0019 & 0.11 & 1,60 & 0.7378 \\
All Within Interactions & & & & \\
Side & Within Subjects & & & \\
Side-by-Treatment & 0.0405 & 0.81 & 3,60 & 0.4938 \\
Side-by-Sex & 0.0051 & 0.31 & 1,60 & 0.5828 \\
Side-by-Treatment-by-Sex & 0.0005 & 0.03 & 1,60 & 0.8699 \\
& 0.0280 & 1.68 & 1,60 & 0.1997 \\
& 0.0091 & 0.54 & 1,60 & 0.4633 \\
\hline
\end{tabular}


Table B-31. Results of mixed-model ANOVA testing for treatment (Edgewise with or without MARA) sex (male, female), and side (left, right TMJ) differences for Axial MLCP L Initial and Axial MLCP R Initial.

\begin{tabular}{lcccc}
\hline \multicolumn{1}{c}{ Source } & SSQ & F ratio & df & P value \\
\hline & Between Subjects & & & \\
All Between & 0.0584 & 1.17 & 3,60 & 0.3293 \\
Treatment & 0.0065 & 0.39 & 1,60 & 0.5346 \\
Sex & 0.0442 & 2.65 & 1,60 & 0.1086 \\
Treatment-by-Sex & 0.0104 & 0.62 & 1,60 & 0.4335 \\
& & & & \\
All Within Interactions & Within Subjects & & & \\
Side & 0.0818 & 1.64 & 3,60 & 0.1905 \\
Side-by-Treatment & 0.0267 & 1.60 & 1,60 & 0.2103 \\
Side-by-Treatment-by-Sex & 0.0552 & 3.31 & 1,60 & 0.0738 \\
\hline
\end{tabular}

Table B-32. Results of mixed-model ANOVA testing for treatment (Edgewise with or without MARA) sex (male, female), and side (left, right TMJ) differences for Axial MLCP L Final and Axial MLCP R Final.

\begin{tabular}{lcccc}
\hline \multicolumn{1}{c}{ Source } & SSQ & F ratio & df & P value \\
\hline & Between Subjects & & & \\
All Between & 0.0714 & 1.43 & 3,60 & 0.2437 \\
Treatment & 0.0027 & 0.16 & 1,60 & 0.6897 \\
Sex & 0.0628 & 3.77 & 1,60 & 0.0570 \\
Treatment-by-Sex & 0.0070 & 0.42 & 1,60 & 0.5202 \\
& & & & \\
All Within Interactions & Within Subjects & & & \\
Side & 0.0932 & 1.86 & 3,60 & 0.1452 \\
Side-by-Treatment & 0.0241 & 1.44 & 1,60 & 0.2342 \\
Side-by-Sex & 0.0356 & 2.14 & 1,60 & 0.1492 \\
Side-by-Treatment-by-Sex & 0.0636 & 3.82 & 1,60 & 0.0554 \\
& 0.0084 & 0.50 & 1,60 & 0.4809 \\
\hline
\end{tabular}


Table B-33. Results of mixed-model ANOVA testing for treatment (Edgewise with or without MARA) sex (male, female), and side (left, right TMJ) differences for Change in Axial MLCP L and Change in Axial MLCP R.

\begin{tabular}{lcccc}
\hline \multicolumn{1}{c}{ Source } & SSQ & F ratio & df & P value \\
\hline & & & & \\
All Between & Between Subjects & & & \\
Treatment & 0.0284 & 0.57 & 3,60 & 0.6378 \\
Sex & 0.0053 & 0.32 & 1,60 & 0.5742 \\
Treatment-by-Sex & 0.0180 & 1.08 & 1,60 & 0.3029 \\
& 0.0017 & 0.10 & 1,60 & 0.7506 \\
All Within Interactions & Within Subjects & & & \\
Side & 0.0191 & 0.38 & 3,60 & 0.7666 \\
Side-by-Treatment & 0.0004 & 0.02 & 1,60 & 0.8779 \\
Side-by-Sex & 0.0007 & 0.04 & 1,60 & 0.8432 \\
Side-by-Treatment-by-Sex & 0.0044 & 0.26 & 1,60 & 0.6089 \\
& 0.0150 & 0.90 & 1,60 & 0.3471 \\
\hline
\end{tabular}

Table B-34. Results of mixed-model ANOVA testing for treatment (Edgewise with or without MARA) sex (male, female), and side (left, right TMJ) differences for Axial CA L Initial and Axial CA R Initial.

\begin{tabular}{lcccc}
\hline \multicolumn{1}{c}{ Source } & SSQ & F ratio & df & P value \\
\hline & Between Subjects & & & \\
All Between & 0.0212 & 0.43 & 3,60 & 0.7358 \\
Treatment & 0.0157 & 0.94 & 1,60 & 0.3357 \\
Sex & 0.0051 & 0.31 & 1,60 & 0.5820 \\
Treatment-by-Sex & 0.0025 & 0.15 & 1,60 & 0.7004 \\
& & & & \\
All Within Interactions & Within Subjects & & & \\
Side & 0.0738 & 1.48 & 3,60 & 0.2299 \\
Side-by-Treatment & 0.0000 & 0.00 & 1,60 & 0.9784 \\
Side-by-Sex & 0.0006 & 0.03 & 1,60 & 0.8554 \\
Side-by-Treatment-by-Sex & 0.0532 & 3.19 & 1,60 & 0.0789 \\
& 0.0151 & 0.90 & 1,60 & 0.3453 \\
\hline
\end{tabular}


Table B-35. Results of mixed-model ANOVA testing for treatment (Edgewise with or without MARA) sex (male, female), and side (left, right TMJ) differences for Axial CA L Final and Axial CA R Final.

\begin{tabular}{lcccc}
\hline \multicolumn{1}{c}{ Source } & SSQ & F ratio & df & P value \\
\hline & Between Subjects & & & \\
All Between & 0.0586 & 1.17 & 3,60 & 0.3278 \\
Treatment & 0.0160 & 0.96 & 1,60 & 0.3317 \\
Sex & 0.0235 & 1.41 & 1,60 & 0.2397 \\
Treatment-by-Sex & 0.0227 & 1.36 & 1,60 & 0.2483 \\
& & & & \\
All Within Interactions & Within Subjects & & & \\
Side & 0.0342 & 0.68 & 3,60 & 0.5649 \\
Side-by-Treatment & 0.0177 & 1.06 & 1,60 & 0.3063 \\
Side-by-Sex & 0.0027 & 0.16 & 1,60 & 0.6889 \\
Side-by-Treatment-by-Sex & 0.0079 & 0.47 & 1,60 & 0.4938 \\
& 0.0211 & 1.27 & 1,60 & 0.2649 \\
\hline
\end{tabular}

Table B-36. Results of mixed-model ANOVA testing for treatment (Edgewise with or without MARA) sex (male, female), and side (left, right TMJ) differences for Change in Axial CA $L$ and Change in Axial CA R.

\begin{tabular}{lcccc}
\hline \multicolumn{1}{c}{ Source } & SSQ & F ratio & df & P value \\
\hline & Between Subjects & & & \\
All Between & 0.0330 & 0.66 & 3,60 & 0.5793 \\
Treatment & 0.0005 & 0.03 & 1,60 & 0.8590 \\
Sex & 0.0113 & 0.68 & 1,60 & 0.4126 \\
Treatment-by-Sex & 0.0191 & 1.15 & 1,60 & 0.2882 \\
& & & & \\
All Within Interactions & Within Subjects & & & \\
Side & 0.0246 & 0.49 & 3,60 & 0.6889 \\
Side-by-Treatment & 0.0306 & 1.83 & 1,60 & 0.1807 \\
Side-by-Sex & 0.0018 & 0.11 & 1,60 & 0.7440 \\
Side-by-Treatment-by-Sex & 0.0220 & 1.32 & 1,60 & 0.2556 \\
& 0.0028 & 0.17 & 1,60 & 0.6843 \\
\hline
\end{tabular}


Table B-37. Results of mixed-model ANOVA testing for treatment (Edgewise with or without MARA) sex (male, female), and side (left, right TMJ) differences for Axial LACP L Initial and Axial LACP R Initial.

\begin{tabular}{lcccc}
\hline \multicolumn{1}{c}{ Source } & SSQ & F ratio & df & P value \\
\hline & Between Subjects & & & \\
All Between & 0.1637 & 3.27 & 3,60 & 0.0271 \\
Treatment & 0.0010 & 0.06 & 1,60 & 0.8054 \\
Sex & 0.1309 & 7.85 & 1,60 & 0.0068 \\
Treatment-by-Sex & 0.0281 & 1.69 & 1,60 & 0.1991 \\
& Within Subjects & & & \\
All Within Interactions & 0.0265 & 0.53 & 3,60 & 0.6640 \\
Side & 0.0493 & 2.96 & 1,60 & 0.0906 \\
Side-by-Treatment & 0.0000 & 0.00 & 1,60 & 0.9568 \\
Side-by-Sex & 0.0026 & 0.16 & 1,60 & 0.6926 \\
Side-by-Treatment-by-Sex & 0.0233 & 1.40 & 1,60 & 0.2418 \\
\hline
\end{tabular}

Table B-38. Results of mixed-model ANOVA testing for treatment (Edgewise with or without MARA) sex (male, female), and side (left, right TMJ) differences for Axial LACP L Final and Axial LACP R Final.

\begin{tabular}{lcccc}
\hline \multicolumn{1}{c}{ Source } & SSQ & F ratio & df & P value \\
\hline & Between Subjects & & & \\
All Between & 0.0731 & 1.46 & 3,60 & 0.2342 \\
Treatment & 0.0708 & 4.25 & 1,60 & 0.0437 \\
Sex & 0.0008 & 0.05 & 1,60 & 0.8304 \\
Treatment-by-Sex & 0.0019 & 0.11 & 1,60 & 0.7378 \\
& & & & \\
All Within Interactions & Within Subjects & & & \\
Side & 0.0405 & 0.81 & 3,60 & 0.4938 \\
Side-by-Treatment & 0.0051 & 0.31 & 1,60 & 0.5828 \\
Side-by-Sex & 0.0005 & 0.03 & 1,60 & 0.8699 \\
Side-by-Treatment-by-Sex & 0.0280 & 1.68 & 1,60 & 0.1997 \\
& 0.0091 & 0.54 & 1,60 & 0.4633 \\
\hline
\end{tabular}


Table B-39. Results of mixed-model ANOVA testing for treatment (Edgewise with or without MARA) sex (male, female), and side (left, right TMJ) differences for Change in Axial LACP L and Change in Axial LACP R.

\begin{tabular}{lcccc}
\hline \multicolumn{1}{c}{ Source } & SSQ & F ratio & df & P value \\
\hline & Between Subjects & & & \\
All Between & 0.0731 & 1.46 & 3,60 & 0.2342 \\
Treatment & 0.0708 & 4.25 & 1,60 & 0.0437 \\
Sex & 0.0008 & 0.05 & 1,60 & 0.8304 \\
Treatment-by-Sex & 0.0019 & 0.11 & 1,60 & 0.7378 \\
& Within Subjects & & & \\
All Within Interactions & 0.0405 & 0.81 & 3,60 & 0.4938 \\
Side & 0.0051 & 0.31 & 1,60 & 0.5828 \\
Side-by-Treatment & 0.0005 & 0.03 & 1,60 & 0.8699 \\
Side-by-Sex & 0.0280 & 1.68 & 1,60 & 0.1997 \\
Side-by-Treatment-by-Sex & 0.0091 & 0.54 & 1,60 & 0.4633 \\
\hline
\end{tabular}

Table B-40. Results of mixed-model ANOVA testing for treatment (Edgewise with or without MARA) sex (male, female), and side (left, right TMJ) differences for Sagittal AJS L Initial and Sagittal AJS R Initial.

\begin{tabular}{lcccc}
\hline \multicolumn{1}{c}{ Source } & SSQ & F ratio & df & P value \\
\hline & Between Subjects & & & \\
All Between & 0.0228 & 0.4568 & 3,60 & 0.7135 \\
Treatment & 0.0178 & 1.0679 & 1,60 & 0.3056 \\
Sex & 0.0015 & 0.0905 & 1,60 & 0.7646 \\
Treatment-by-Sex & 0.0013 & 0.0806 & 1,60 & 0.7775 \\
& & & & \\
All Within Interactions & Within Subjects & & & \\
Side & 0.0102 & 0.2045 & 3,60 & 0.8929 \\
Side-by-Treatment & 0.0103 & 0.6163 & 1,60 & 0.4355 \\
Side-by-Sex & 0.0031 & 0.1841 & 1,60 & 0.6694 \\
Side-by-Treatment-by-Sex & 0.0026 & 0.1544 & 1,60 & 0.6958 \\
& 0.0051 & 0.3039 & 1,60 & 0.5835 \\
\hline
\end{tabular}


Table B-41. Results of mixed-model ANOVA testing for treatment (Edgewise with or without MARA) sex (male, female), and side (left, right TMJ) differences for Sagittal AJS L Final and Sagittal AJS R Final.

\begin{tabular}{lcccc}
\hline \multicolumn{1}{c}{ Source } & SSQ & F ratio & df & P value \\
\hline & Between Subjects & & & \\
All Between & 0.0466 & 0.9311 & 3,60 & 0.4313 \\
Treatment & 0.0166 & 0.9936 & 1,60 & 0.3229 \\
Sex & 0.0121 & 0.7286 & 1,60 & 0.3967 \\
Treatment-by-Sex & 0.0119 & 0.7157 & 1,60 & 0.4009 \\
& & & & \\
All Within Interactions & Within Subjects & & & \\
Side & 0.0385 & 0.7705 & 3,60 & 0.5150 \\
Side-by-Treatment & 0.0893 & 5.3587 & 1,60 & 0.0241 \\
Side-by-Sex & 0.0034 & 0.2062 & 1,60 & 0.6514 \\
Side-by-Treatment-by-Sex & 0.0058 & 0.3475 & 1,60 & 0.5577 \\
& 0.0314 & 1.8855 & 1,60 & 0.1748 \\
\hline
\end{tabular}

Table B-42. Results of mixed-model ANOVA testing for treatment (Edgewise with or without MARA) sex (male, female), and side (left, right TMJ) differences for Change in Sagittal AJS L and Change in Sagittal AJS R.

\begin{tabular}{lcccc}
\hline \multicolumn{1}{c}{ Source } & SSQ & F ratio & df & P value \\
\hline & Between Subjects & & & \\
All Between & 0.0102 & 0.204 & 3,60 & 0.8932 \\
Treatment & 0.0004 & 0.0253 & 1,60 & 0.8741 \\
Sex & 0.0048 & 0.2866 & 1,60 & 0.5944 \\
Treatment-by-Sex & 0.0050 & 0.2992 & 1,60 & 0.5864 \\
& & & & \\
All Within Interactions & Within Subjects & & & \\
Side & 0.0262 & 0.5238 & 3,60 & 0.6676 \\
Side-by-Treatment & 0.0301 & 1.8038 & 1,60 & 0.1843 \\
Side-by-Sex & 0.0093 & 0.5597 & 1,60 & 0.4573 \\
Side-by-Treatment-by-Sex & 0.0116 & 0.6971 & 1,60 & 0.4071 \\
& 0.0088 & 0.5264 & 1,60 & 0.4710 \\
\hline
\end{tabular}


Table B-43. Results of mixed-model ANOVA testing for treatment (Edgewise with or without MARA) sex (male, female), and side (left, right TMJ) differences for Sagittal MJS L Initial and Sagittal MJS R Initial.

\begin{tabular}{lcccc}
\hline \multicolumn{1}{c}{ Source } & SSQ & F ratio & df & P value \\
\hline & Between Subjects & & & \\
All Between & 0.3361 & 6.72 & 3,60 & 0.0006 \\
Treatment & 0.2766 & 16.59 & 1,60 & 0.0001 \\
Sex & 0.0219 & 1.31 & 1,60 & 0.2562 \\
Treatment-by-Sex & 0.0048 & 0.29 & 1,60 & 0.5926 \\
& & & & \\
All Within Interactions & Within Subjects & & & \\
Side & 0.0057 & 0.11 & 3,60 & 0.9518 \\
Side-by-Treatment & 0.0475 & 2.85 & 1,60 & 0.0967 \\
Side-by-Sex & 0.0045 & 0.27 & 1,60 & 0.6055 \\
Side-by-Treatment-by-Sex & 0.0000 & 0.00 & 1,60 & 0.9838 \\
& 0.0010 & 0.06 & 1,60 & 0.8079 \\
\hline
\end{tabular}

Table B-44. Results of mixed-model ANOVA testing for treatment (Edgewise with or without MARA) sex (male, female), and side (left, right TMJ) differences for Sagittal MJS L Final and Sagittal AJS R Final.

\begin{tabular}{lcccc}
\hline \multicolumn{1}{c}{ Source } & SSQ & F ratio & df & P value \\
\hline & & & & \\
All Between & Between Subjects & & & \\
Treatment & 0.1112 & 2.22 & 3,60 & 0.0945 \\
Sex & 0.0176 & 1.05 & 1,60 & 0.3088 \\
Treatment-by-Sex & 0.0689 & 4.13 & 1,60 & 0.0464 \\
& 0.0098 & 0.59 & 1,60 & 0.4461 \\
All Within Interactions & & & & \\
Side & Within Subjects & & & \\
Side-by-Treatment & 0.0308 & 0.62 & 3,60 & 0.6076 \\
Side-by-Sex & 0.1511 & 9.07 & 1,60 & 0.0038 \\
Side-by-Treatment-by-Sex & 0.0004 & 0.02 & 1,60 & 0.8833 \\
& 0.0263 & 1.58 & 1,60 & 0.2143 \\
& 0.0020 & 0.12 & 1,60 & 0.7290 \\
\hline
\end{tabular}


Table B-45. Results of mixed-model ANOVA testing for treatment (Edgewise with or without MARA) sex (male, female), and side (left, right TMJ) differences for Change in Sagittal MJS L and Change in Sagittal MJS R.

\begin{tabular}{lcccc}
\hline \multicolumn{1}{c}{ Source } & SSQ & F ratio & df & P value \\
\hline & Between Subjects & & & \\
All Between & 0.1289 & 2.58 & 3,60 & 0.0620 \\
Treatment & 0.1076 & 6.45 & 1,60 & 0.0137 \\
Sex & 0.0329 & 1.97 & 1,60 & 0.1654 \\
Treatment-by-Sex & 0.0031 & 0.19 & 1,60 & 0.6677 \\
& & & & \\
All Within Interactions & Within Subjects & & & \\
Side & 0.0179 & 0.36 & 3,60 & 0.7832 \\
Side-by-Treatment & 0.0117 & 0.70 & 1,60 & 0.4064 \\
Side-by-Sex & 0.0016 & 0.10 & 1,60 & 0.7559 \\
Side-by-Treatment-by-Sex & 0.0135 & 0.81 & 1,60 & 0.3711 \\
& 0.0034 & 0.20 & 1,60 & 0.6528 \\
\hline
\end{tabular}

Table B-46. Results of mixed-model ANOVA testing for treatment (Edgewise with or without MARA) sex (male, female), and side (left, right TMJ) differences for Sagittal PJS L Initial and Sagittal PJS R Initial.

\begin{tabular}{lcccc}
\hline \multicolumn{1}{c}{ Source } & SSQ & F ratio & df & P value \\
\hline & Between Subjects & & & \\
All Between & 0.0209 & 0.42 & 3,60 & 0.7402 \\
Treatment & 0.0001 & 0.01 & 1,60 & 0.9357 \\
Sex & 0.0099 & 0.59 & 1,60 & 0.4445 \\
Treatment-by-Sex & 0.0104 & 0.62 & 1,60 & 0.4336 \\
& & & & \\
All Within Interactions & Within Subjects & & & \\
Side & 0.0234 & 0.47 & 3,60 & 0.7052 \\
Side-by-Treatment & 0.0005 & 0.03 & 1,60 & 0.8693 \\
Side-by-Sex & 0.0081 & 0.49 & 1,60 & 0.4882 \\
Side-by-Treatment-by-Sex & 0.0105 & 0.63 & 1,60 & 0.4296 \\
\hline
\end{tabular}


Table B-47. Results of mixed-model ANOVA testing for treatment (Edgewise with or without MARA) sex (male, female), and side (left, right TMJ) differences for Sagittal PJS L Final and Sagittal PJS R Final.

\begin{tabular}{lcccc}
\hline \multicolumn{1}{c}{ Source } & SSQ & F ratio & df & P value \\
\hline & Between Subjects & & & \\
All Between & 0.0287 & 0.57 & 3,60 & 0.6338 \\
Treatment & 0.0196 & 1.18 & 1,60 & 0.2826 \\
Sex & 0.0032 & 0.19 & 1,60 & 0.6627 \\
Treatment-by-Sex & 0.0084 & 0.50 & 1,60 & 0.4816 \\
& & & & \\
All Within Interactions & Within Subjects & & & \\
Side & 0.0737 & 1.47 & 3,60 & 0.2308 \\
All Within Interactions & 0.0003 & 0.02 & 1,60 & 0.8894 \\
Side & 0.0676 & 4.06 & 1,60 & 0.0485 \\
Side-by-Treatment & 0.0010 & 0.06 & 1,60 & 0.8074 \\
& 0.0007 & 0.04 & 1,60 & 0.8381 \\
\hline
\end{tabular}

Table B-48. Results of mixed-model ANOVA testing for treatment (Edgewise with or without MARA) sex (male, female), and side (left, right TMJ) differences for Change in Sagittal PJS L and Change in Sagittal PJS R.

\begin{tabular}{lcccc}
\hline \multicolumn{1}{c}{ Source } & SSQ & F ratio & df & P value \\
\hline & Between Subjects & & & \\
All Between & 0.0330 & 0.66 & 3,60 & 0.5793 \\
Treatment & 0.0005 & 0.03 & 1,60 & 0.8590 \\
Sex & 0.0113 & 0.68 & 1,60 & 0.4126 \\
Treatment-by-Sex & 0.0191 & 1.15 & 1,60 & 0.2882 \\
& & & & \\
All Within Interactions & Within Subjects & & & \\
Side & 0.0246 & 0.49 & 3,60 & 0.6889 \\
Side-by-Treatment & 0.0306 & 1.83 & 1,60 & 0.1807 \\
Side-by-Sex & 0.0018 & 0.11 & 1,60 & 0.7440 \\
Side-by-Treatment-by-Sex & 0.0220 & 1.32 & 1,60 & 0.2556 \\
\hline
\end{tabular}


Table B-49. Results of mixed-model ANOVA testing for treatment (Edgewise with or without MARA) sex (male, female), and side (left, right TMJ) differences for Sagittal FW L Initial and Sagittal FW R Initial.

\begin{tabular}{lcccc}
\hline \multicolumn{1}{c}{ Source } & SSQ & F ratio & df & P value \\
\hline & Between Subjects & & & \\
All Between & 0.0627 & 1.25 & 3,60 & 0.2986 \\
Treatment & 0.0580 & 3.48 & 1,60 & 0.0670 \\
Sex & 0.0004 & 0.02 & 1,60 & 0.8834 \\
Treatment-by-Sex & 0.0045 & 0.27 & 1,60 & 0.6048 \\
& Within Subjects & & & \\
All Within Interactions & 0.0869 & 1.74 & 3,60 & 0.1690 \\
Side & 0.0016 & 0.09 & 1,60 & 0.7610 \\
Side-by-Treatment & 0.0837 & 5.02 & 1,60 & 0.0288 \\
Side-by-Sex & 0.0000 & 0.00 & 1,60 & 0.9935 \\
Side-by-Treatment-by-Sex & 0.0013 & 0.08 & 1,60 & 0.7808 \\
\hline
\end{tabular}

Table B-50. Results of mixed-model ANOVA testing for treatment (Edgewise with or without MARA) sex (male, female), and side (left, right TMJ) differences for Sagittal FW L Final and Sagittal FW R Final.

\begin{tabular}{lcccc}
\hline \multicolumn{1}{c}{ Source } & SSQ & F ratio & df & P value \\
\hline & & & & \\
All Between & Between Subjects & & & \\
Treatment & 0.1135 & 2.27 & 3,60 & 0.0896 \\
Sex & 0.0759 & 4.56 & 1,60 & 0.0369 \\
Treatment-by-Sex & 0.0515 & 3.09 & 1,60 & 0.0839 \\
& 0.0021 & 0.12 & 1,60 & 0.7265 \\
All Within Interactions & & & & \\
Side & Within Subjects & & & \\
Side-by-Treatment & 0.2212 & 4.42 & 3,60 & 0.0071 \\
Side-by-Sex & 0.0106 & 0.64 & 1,60 & 0.4278 \\
Side-by-Treatment-by-Sex & 0.2179 & 13.07 & 1,60 & 0.0006 \\
& 0.0116 & 0.69 & 1,60 & 0.4083 \\
& 0.0027 & 0.16 & 1,60 & 0.6863 \\
\hline
\end{tabular}


Table B-51. Results of mixed-model ANOVA testing for treatment (Edgewise with or without MARA) sex (male, female), and side (left, right TMJ) differences for Change in Sagittal FW L and Change in Sagittal FW R.

\begin{tabular}{lcccc}
\hline \multicolumn{1}{c}{ Source } & SSQ & F ratio & df & P value \\
\hline & Between Subjects & & & \\
All Between & 0.1406 & 2.81 & 3,60 & 0.0469 \\
Treatment & 0.0002 & 0.01 & 1,60 & 0.9107 \\
Sex & 0.1012 & 6.07 & 1,60 & 0.0166 \\
Treatment-by-Sex & 0.0342 & 2.05 & 1,60 & 0.1575 \\
& & & & \\
All Within Interactions & Within Subjects & & & \\
Side & 0.0179 & 0.36 & 3,60 & 0.7839 \\
Side-by-Treatment & 0.0025 & 0.15 & 1,60 & 0.6989 \\
Side-by-Sex & 0.0114 & 0.68 & 1,60 & 0.4113 \\
Side-by-Treatment-by-Sex & 0.0092 & 0.55 & 1,60 & 0.4600 \\
& 0.0001 & 0.00 & 1,60 & 0.9505 \\
\hline
\end{tabular}

Table B-52. Results of mixed-model ANOVA testing for treatment (Edgewise with or without MARA) sex (male, female), and side (left, right TMJ) differences for Sagittal FD L Initial and Sagittal FD R Initial.

\begin{tabular}{lcccc}
\hline \multicolumn{1}{c}{ Source } & SSQ & F ratio & df & P value \\
\hline & Between Subjects & & & \\
All Between & 0.1379 & 2.76 & 3,60 & 0.0501 \\
Treatment & 0.1104 & 6.62 & 1,60 & 0.0126 \\
Sex & 0.0093 & 0.56 & 1,60 & 0.4582 \\
Treatment-by-Sex & 0.0047 & 0.28 & 1,60 & 0.5980 \\
& & & & \\
All Within Interactions & Within Subjects & & & \\
Side & 0.0439 & 0.88 & 3,60 & 0.4578 \\
Side-by-Treatment & 0.0265 & 1.59 & 1,60 & 0.2126 \\
Side-by-Sex & 0.0298 & 1.79 & 1,60 & 0.1866 \\
Side-by-Treatment-by-Sex & 0.0076 & 0.46 & 1,60 & 0.5025 \\
& 0.0007 & 0.04 & 1,60 & 0.8400 \\
\hline
\end{tabular}


Table B-53. Results of mixed-model ANOVA testing for treatment (Edgewise with or without MARA) sex (male, female), and side (left, right TMJ) differences for Sagittal FD L Final and Sagittal FD R Final.

\begin{tabular}{lcccc}
\hline \multicolumn{1}{c}{ Source } & SSQ & F ratio & df & P value \\
\hline & Between Subjects & & & \\
All Between & 0.0523 & 1.05 & 3,60 & 0.3791 \\
Treatment & 0.0400 & 2.40 & 1,60 & 0.1267 \\
Sex & 0.0020 & 0.12 & 1,60 & 0.7324 \\
Treatment-by-Sex & 0.0064 & 0.38 & 1,60 & 0.5392 \\
& & & & \\
All Within Interactions & Within Subjects & & & \\
Side & 0.0206 & 0.41 & 3,60 & 0.7456 \\
Side-by-Treatment & 0.0086 & 0.52 & 1,60 & 0.4755 \\
Side-by-Sex & 0.0137 & 0.82 & 1,60 & 0.3682 \\
Side-by-Treatment-by-Sex & 0.0040 & 0.24 & 1,60 & 0.6267 \\
& 0.0000 & 0.00 & 1,60 & 0.9632 \\
\hline
\end{tabular}

Table B-54. Results of mixed-model ANOVA testing for treatment (Edgewise with or without MARA) sex (male, female), and side (left, right TMJ) differences for Change in Sagittal FD L and Change in Sagittal FD R.

\begin{tabular}{lcccc}
\hline \multicolumn{1}{c}{ Source } & SSQ & F ratio & df & P value \\
\hline & & & & \\
All Between & Between Subjects & & & \\
Treatment & 0.0596 & 1.19 & 3,60 & 0.3207 \\
Sex & 0.0447 & 2.68 & 1,60 & 0.1069 \\
Treatment-by-Sex & 0.0073 & 0.44 & 1,60 & 0.5107 \\
& 0.0006 & 0.04 & 1,60 & 0.8503 \\
All Within Interactions & Within Subjects & & & \\
Side & 0.0028 & 0.06 & 1,60 & 0.9827 \\
Side-by-Treatment & 0.0033 & 0.20 & 1,60 & 0.6590 \\
Side-by-Sex & 0.0018 & 0.11 & 1,60 & 0.7416 \\
Side-by-Treatment-by-Sex & 0.0003 & 0.02 & 1,60 & 0.8910 \\
& 0.0003 & 0.02 & 1,60 & 0.8910 \\
\hline
\end{tabular}


Table B-55. Results of mixed-model ANOVA testing for treatment (Edgewise with or without MARA) sex (male, female), and side (left, right TMJ) differences for Sagittal CHW L Initial and Sagittal CHW R Initial.

\begin{tabular}{lcccc}
\hline \multicolumn{1}{c}{ Source } & SSQ & F ratio & df & P value \\
\hline & Between Subjects & & & \\
All Between & 0.1111 & 2.22 & 3,60 & 0.0947 \\
Treatment & 0.0196 & 1.18 & 1,60 & 0.2822 \\
Sex & 0.0650 & 3.90 & 1,60 & 0.0528 \\
Treatment-by-Sex & 0.0325 & 1.95 & 1,60 & 0.1676 \\
& & & & \\
All Within Interactions & Within Subjects & & & \\
Side & 0.1758 & 3.52 & 3,60 & 0.0204 \\
Side-by-Treatment & 0.0396 & 2.38 & 1,60 & 0.1283 \\
Side-by-Sex & 0.0491 & 2.95 & 1,60 & 0.0911 \\
Side-by-Treatment-by-Sex & 0.0544 & 3.26 & 1,60 & 0.0759 \\
& 0.0560 & 3.36 & 1,60 & 0.0718 \\
\hline
\end{tabular}

Table B-56. Results of mixed-model ANOVA testing for treatment (Edgewise with or without MARA) sex (male, female), and side (left, right TMJ) differences for Sagittal CHW L Final and Sagittal CHW R Final.

\begin{tabular}{lcccc}
\hline \multicolumn{1}{c}{ Source } & SSQ & F ratio & df & P value \\
\hline & Between Subjects & & & \\
All Between & 0.0498 & 1.00 & 3,60 & 0.4014 \\
Treatment & 0.0195 & 1.17 & 1,60 & 0.2832 \\
Sex & 0.0318 & 1.91 & 1,60 & 0.1723 \\
Treatment-by-Sex & 0.0061 & 0.36 & 1,60 & 0.5484 \\
& & & & \\
All Within Interactions & Within Subjects & & & \\
Side & 0.1577 & 3.15 & 3,60 & 0.0313 \\
Side-by-Treatment & 0.0318 & 1.91 & 1,60 & 0.1723 \\
Side-by-Sex & 0.1127 & 6.76 & 1,60 & 0.0117 \\
Side-by-Treatment-by-Sex & 0.0214 & 1.28 & 1,60 & 0.2621 \\
& 0.0049 & 0.29 & 1,60 & 0.5910 \\
\hline
\end{tabular}


Table B-57. Results of mixed-model ANOVA testing for treatment (Edgewise with or without MARA) sex (male, female), and side (left, right TMJ) differences for Change in Sagittal CHW L and Change in Sagittal CHW R.

\begin{tabular}{lcccc}
\hline \multicolumn{1}{c}{ Source } & SSQ & F ratio & df & P value \\
\hline & Between Subjects & & & \\
All Between & 0.2176 & 4.35 & 3,60 & 0.0077 \\
Treatment & 0.0008 & 0.05 & 1,60 & 0.8247 \\
Sex & 0.0085 & 0.51 & 1,60 & 0.4783 \\
Treatment-by-Sex & 0.2044 & 12.27 & 1,60 & 0.0009 \\
& & & & \\
All Within Interactions & Within Subjects & & & \\
Side & 0.0519 & 1.04 & 3,60 & 0.3827 \\
Side-by-Treatment & 0.0009 & 0.05 & 1,60 & 0.8192 \\
Side-by-Sex & 0.0122 & 0.73 & 1,60 & 0.3949 \\
Side-by-Treatment-by-Sex & 0.0101 & 0.60 & 1,60 & 0.4400 \\
& 0.0339 & 2.03 & 1,60 & 0.1591 \\
\hline
\end{tabular}

Table B-58. Results of mixed-model ANOVA testing for treatment (Edgewise with or without MARA) sex (male, female), and side (left, right TMJ) differences for Sagittal CHH L Initial and Sagittal CHH R Initial.

\begin{tabular}{lcccc}
\hline \multicolumn{1}{c}{ Source } & SSQ & F ratio & df & P value \\
\hline & & & & \\
All Between & Between Subjects & & & \\
Treatment & 0.1222 & 2.44 & 3,60 & 0.0727 \\
Sex & 0.0393 & 2.36 & 1,60 & 0.1299 \\
Treatment-by-Sex & 0.0958 & 5.75 & 1,60 & 0.0197 \\
& 0.0023 & 0.14 & 1,60 & 0.7120 \\
All Within Interactions & & & & \\
Side & Within Subjects & & & \\
Side-by-Treatment & 0.0542 & 1.08 & 3,60 & 0.3629 \\
Side-by-Sex & 0.0021 & 0.13 & 1,60 & 0.7237 \\
Side-by-Treatment-by-Sex & 0.0173 & 1.04 & 1,60 & 0.3118 \\
& 0.0091 & 0.55 & 1,60 & 0.4629 \\
& 0.0223 & 1.34 & 1,60 & 0.2519 \\
\hline
\end{tabular}


Table B-59. Results of mixed-model ANOVA testing for treatment (Edgewise with or without MARA) sex (male, female), and side (left, right TMJ) differences for Sagittal CHH L Final and Sagittal CHH R Final.

\begin{tabular}{lcccc}
\hline \multicolumn{1}{c}{ Source } & SSQ & F ratio & df & P value \\
\hline & & & & \\
All Between & Between Subjects & & & \\
Treatment & 0.1426 & 2.85 & 3,60 & 0.0447 \\
Sex & 0.0634 & 3.81 & 1,60 & 0.0558 \\
Treatment-by-Sex & 0.1001 & 6.01 & 1,60 & 0.0172 \\
& 0.0001 & 0.01 & 1,60 & 0.9367 \\
All Within Interactions & Within Subjects & & & \\
Side & 0.0794 & 1.59 & 3,60 & 0.2015 \\
Side-by-Treatment & 0.0399 & 2.40 & 1,60 & 0.1269 \\
Side-by-Sex & 0.0586 & 3.52 & 1,60 & 0.0656 \\
Side-by-Treatment-by-Sex & 0.0108 & 0.65 & 1,60 & 0.4239 \\
& 0.0001 & 0.01 & 1,60 & 0.9270 \\
\hline
\end{tabular}

Table B-60. Results of mixed-model ANOVA testing for treatment (Edgewise with or without MARA) sex (male, female), and side (left, right TMJ) differences for Change in Sagittal CHH L and Change in Sagittal CHH R.

\begin{tabular}{lcccc}
\hline \multicolumn{1}{c}{ Source } & SSQ & F ratio & df & P value \\
\hline & & & & \\
All Between & Between Subjects & & & \\
Treatment & 0.0065 & 0.13 & 3,60 & 0.9415 \\
Sex & 0.0038 & 0.23 & 1,60 & 0.6327 \\
Treatment-by-Sex & 0.0000 & + & 1,60 & 0.9843 \\
& 0.0026 & 0.15 & 1,60 & 0.6970 \\
All Within Interactions & & & & \\
Side & Within Subjects & & & \\
Side-by-Treatment & 0.0291 & 0.58 & 3,60 & 0.6295 \\
Side-by-Sex & 0.0233 & 1.40 & 1,60 & 0.2421 \\
Side-by-Treatment-by-Sex & 0.0196 & 1.17 & 1,60 & 0.2828 \\
& 0.0014 & 0.08 & 1,60 & 0.7743 \\
& 0.0060 & 0.36 & 1,60 & 0.5514 \\
\hline
\end{tabular}


Table B-61. Results of mixed-model ANOVA testing for treatment (Edgewise with or without MARA) sex (male, female), and side (left, right TMJ) differences for Sagittal CH L Initial and Sagittal CH R Initial.

\begin{tabular}{lcccc}
\hline \multicolumn{1}{c}{ Source } & SSQ & F ratio & df & P value \\
\hline & Between Subjects & & & \\
All Between & 0.2124 & 4.25 & 3,60 & 0.0087 \\
Treatment & 0.1780 & 10.68 & 1,60 & 0.0018 \\
Sex & 0.1780 & 10.68 & 1,60 & 0.0018 \\
Treatment-by-Sex & 0.0000 & 0.00 & 1,60 & 0.9772 \\
& & & & \\
All Within Interactions & Within Subjects & & & \\
Side & 0.0044 & 0.09 & & 0.9668 \\
Side-by-Treatment & 0.0101 & 0.61 & 3,60 & 0.4384 \\
Side-by-Sex & 0.0029 & 0.18 & 1,60 & 0.6755 \\
Side-by-Treatment-by-Sex & 0.0005 & 0.03 & 1,60 & 0.8565 \\
& 0.0003 & 0.02 & 1,60 & 0.8857 \\
\hline
\end{tabular}

Table B-62. Results of mixed-model ANOVA testing for treatment (Edgewise with or without MARA) sex (male, female), and side (left, right TMJ) differences for Sagittal CH L Final and Sagittal CH R Final.

\begin{tabular}{lcccc}
\hline \multicolumn{1}{c}{ Source } & SSQ & F ratio & df & P value \\
\hline & Between Subjects & & & \\
All Between & 0.0257 & 0.51 & 3,60 & 0.6743 \\
Treatment & 0.0171 & 1.03 & 1,60 & 0.3149 \\
Sex & 0.0026 & 0.16 & 1,60 & 0.6923 \\
Treatment-by-Sex & 0.0032 & 0.19 & 1,60 & 0.6640 \\
& & & & \\
All Within Interactions & Within Subjects & & & \\
Side & 0.0182 & 0.36 & & 0.7792 \\
Side-by-Treatment & 0.0002 & 0.01 & 3,60 & 0.9077 \\
Side-by-Sex & 0.0042 & 0.25 & 1,60 & 0.6182 \\
Side-by-Treatment-by-Sex & 0.0114 & 0.68 & 1,60 & 0.4117 \\
\hline
\end{tabular}


Table B-63. Results of mixed-model ANOVA testing for treatment (Edgewise with or without MARA) sex (male, female), and side (left, right TMJ) differences for Change in Sagittal CH L and Change in Sagittal CH R.

\begin{tabular}{lcccc}
\hline \multicolumn{1}{c}{ Source } & SSQ & F ratio & df & P value \\
\hline & Between Subjects & & & \\
All Between & 0.2086 & 4.17 & 3,60 & 0.0095 \\
Treatment & 0.1737 & 10.42 & 1,60 & 0.0020 \\
Sex & 0.0080 & 0.48 & 1,60 & 0.4904 \\
Treatment-by-Sex & 0.0105 & 0.63 & 1,60 & 0.4299 \\
& Within Subjects & & & \\
All Within Interactions & 0.0139 & 0.28 & 3,60 & 0.8411 \\
Side & 0.0185 & 1.11 & 1,60 & 0.2957 \\
Side-by-Treatment & 0.0004 & 0.02 & 1,60 & 0.8831 \\
Side-by-Sex & 0.0117 & 0.70 & 1,60 & 0.4045 \\
Side-by-Treatment-by-Sex & 0.0010 & 0.06 & 1,60 & 0.8051 \\
\hline
\end{tabular}

Table B-64. Results of mixed-model ANOVA testing for treatment (Edgewise with or without MARA) sex (male, female), and side (left, right TMJ) differences for Sagittal EAM-AE L Initial and Sagittal EAM-AE R Initial.

\begin{tabular}{lcccc}
\hline \multicolumn{1}{c}{ Source } & SSQ & F ratio & df & P value \\
\hline & & & & \\
All Between & Between Subjects & & & \\
Treatment & 0.1104 & 2.21 & 3,60 & 0.0965 \\
Sex & 0.0901 & 5.40 & 1,60 & 0.0235 \\
Treatment-by-Sex & 0.0099 & 0.59 & 1,60 & 0.4442 \\
& 0.0187 & 1.12 & 1,60 & 0.2934 \\
All Within Interactions & & & & \\
Side & Within Subjects & & & \\
Side-by-Treatment & 0.2882 & 5.76 & 3,60 & 0.0016 \\
Side-by-Sex & 0.1493 & 8.96 & 1,60 & 0.0040 \\
Side-by-Treatment-by-Sex & 0.2874 & 17.24 & 1,60 & 0.0001 \\
& 0.0045 & 0.27 & 1,60 & 0.6066 \\
& 0.0007 & 0.04 & 1,60 & 0.8356 \\
\hline
\end{tabular}


Table B-65. Results of mixed-model ANOVA testing for treatment (Edgewise with or without MARA) sex (male, female), and side (left, right TMJ) differences for Sagittal EAM-AE L Final and Sagittal EAM-AE R Final.

\begin{tabular}{lcccr}
\hline \multicolumn{1}{c}{ Source } & SSQ & F ratio & df & P value \\
\hline & Between Subjects & & & \\
All Between & 0.0809 & 1.62 & 3,60 & 0.1945 \\
Treatment & 0.0693 & 4.16 & 1,60 & 0.0459 \\
Sex & 0.0182 & 1.09 & 1,60 & 0.2998 \\
Treatment-by-Sex & 0.0032 & 0.19 & 1,60 & 0.6615 \\
& & & & \\
All Within Interactions & Within Subjects & & & \\
Side & 0.3346 & 6.69 & 3,60 & 0.0006 \\
Side-by-Treatment & 0.0266 & 1.59 & 1,60 & 0.2116 \\
Side-by-Sex & 0.3199 & 19.19 & 1,60 & $<0.0001$ \\
Side-by-Treatment-by-Sex & 0.0210 & 1.26 & 1,60 & 0.2660 \\
& 0.0131 & 0.79 & 1,60 & 0.3784 \\
\hline
\end{tabular}

Table B-66. Results of mixed-model ANOVA testing for treatment (Edgewise with or without MARA) sex (male, female), and side (left, right TMJ) differences for Change in Sagittal EAM AE $L$ and Change in Sagittal EAM AE R.

\begin{tabular}{lcccc}
\hline \multicolumn{1}{c}{ Source } & SSQ & F ratio & df & P value \\
\hline & Between Subjects & & & \\
All Between & 0.0386 & 0.77 & 3,60 & 0.5147 \\
Treatment & 0.0028 & 0.17 & 1,60 & 0.6850 \\
Sex & 0.0074 & 0.44 & 1,60 & 0.5080 \\
Treatment-by-Sex & 0.0258 & 1.55 & 1,60 & 0.2178 \\
& & & & \\
All Within Interactions & Within Subjects & & & \\
Side & 0.0125 & 0.25 & 3,60 & 0.8606 \\
Side-by-Treatment & 0.0656 & 3.94 & 1,60 & 0.0519 \\
Side-by-Sex & 0.0018 & 0.11 & 1,60 & 0.7416 \\
Side-by-Treatment-by-Sex & 0.0040 & 0.24 & 1,60 & 0.6261 \\
& 0.0060 & 0.36 & 1,60 & 0.5521 \\
\hline
\end{tabular}


Table B-67. Results of mixed-model ANOVA testing for treatment (Edgewise with or without MARA) sex (male, female), and side (left, right TMJ) differences for Sagittal EAM-C L Initial and Sagittal EAM-C R Initial.

\begin{tabular}{lcccr}
\hline \multicolumn{1}{c}{ Source } & SSQ & F ratio & df & P value \\
\hline & & & & \\
All Between & Between Subjects & & & \\
Treatment & 0.0331 & 0.66 & 3,60 & 0.5783 \\
Sex & 0.0028 & 0.17 & 1,60 & 0.6828 \\
Treatment-by-Sex & 0.0006 & 0.04 & 1,60 & 0.8515 \\
& 0.0304 & 1.82 & 1,60 & 0.1820 \\
All Within Interactions & Within Subjects & & & \\
Side & 0.4099 & 8.20 & 3,60 & 0.0001 \\
Side-by-Treatment & 0.1383 & 8.30 & 1,60 & 0.0055 \\
Side-by-Sex & 0.3929 & 23.57 & 1,60 & $<0.0001$ \\
Side-by-Treatment-by-Sex & 0.0002 & 0.01 & 1,60 & 0.9147 \\
& 0.0038 & 0.23 & 1,60 & 0.6333 \\
\hline
\end{tabular}

Table B-68. Results of mixed-model ANOVA testing for treatment (Edgewise with or without MARA) sex (male, female), and side (left, right TMJ) differences for Sagittal EAM-C L Final and Sagittal EAM-C R Final.

\begin{tabular}{lcccc}
\hline \multicolumn{1}{c}{ Source } & SSQ & F ratio & df & P value \\
\hline & & & & \\
All Between & Between Subjects & & & \\
Treatment & 0.0603 & 1.21 & 3,60 & 0.3150 \\
Sex & 0.0446 & 2.68 & 1,60 & 0.1070 \\
Treatment-by-Sex & 0.0005 & 0.03 & 1,60 & 0.8644 \\
& 0.0127 & 0.76 & 1,60 & 0.3871 \\
All Within Interactions & Within Subjects & & & \\
Side & 0.1012 & 2.02 & 3,60 & 0.1202 \\
Side-by-Treatment & 0.0004 & 0.02 & 1,60 & 0.8766 \\
Side-by-Sex & 0.0493 & 2.96 & 1,60 & 0.0905 \\
Side-by-Treatment-by-Sex & 0.0555 & 3.33 & 1,60 & 0.0731 \\
& 0.0124 & 0.74 & 1,60 & 0.3922 \\
\hline
\end{tabular}


Table B-69. Results of mixed-model ANOVA testing for treatment (Edgewise with or without MARA) sex (male, female), and side (left, right TMJ) differences for Change in Sagittal EAM C L and Change in Sagittal EAM C R.

\begin{tabular}{lcccc}
\hline \multicolumn{1}{c}{ Source } & SSQ & F ratio & df & P value \\
\hline & & & & \\
All Between & Between Subjects & & & \\
Treatment & 0.0751 & 1.50 & 3,60 & 0.2230 \\
Sex & 0.0586 & 3.52 & 1,60 & 0.0656 \\
Treatment-by-Sex & 0.0045 & 0.27 & 1,60 & 0.6071 \\
& 0.0054 & 0.33 & 1,60 & 0.5704 \\
All Within Interactions & Within Subjects & & & \\
Side & 0.1258 & 2.52 & 3,60 & 0.0668 \\
Side-by-Treatment & 0.0468 & 2.81 & 1,60 & 0.0991 \\
Side-by-Sex & 0.0326 & 1.96 & 1,60 & 0.1669 \\
Side-by-Treatment-by-Sex & 0.0571 & 3.42 & 1,60 & 0.0692 \\
& 0.0219 & 1.31 & 1,60 & 0.2563 \\
\hline
\end{tabular}

Table B-70. Results of mixed-model ANOVA testing for treatment (Edgewise with or without MARA) sex (male, female), and side (left, right TMJ) differences for Sagittal EAM-P L Initial and Sagittal EAM-P R Initial.

\begin{tabular}{lcccc}
\hline \multicolumn{1}{c}{ Source } & SSQ & F ratio & df & P value \\
\hline & Between Subjects & & & \\
All Between & 0.0115 & 0.23 & 3,60 & 0.8748 \\
Treatment & 0.0011 & 0.06 & 1,60 & 0.8020 \\
Sex & 0.0000 & 0.00 & 1,60 & 0.9883 \\
Treatment-by-Sex & 0.0104 & 0.62 & 1,60 & 0.4327 \\
& & & & \\
All Within Interactions & Within Subjects & & & \\
Side & 0.1421 & 2.84 & 3,60 & 0.0452 \\
Side-by-Treatment & 0.1328 & 7.97 & 1,60 & 0.0064 \\
Side-by-Sex & 0.1155 & 6.93 & 1,60 & 0.0108 \\
Side-by-Treatment-by-Sex & 0.0270 & 1.62 & 1,60 & 0.2082 \\
& 0.0157 & 0.94 & 1,60 & 0.3362 \\
\hline
\end{tabular}


Table B-71. Results of mixed-model ANOVA testing for treatment (Edgewise with or without MARA) sex (male, female), and side (left, right TMJ) differences for Sagittal EAM-P L Final and Sagittal EAM-P R Final.

\begin{tabular}{lcccc}
\hline \multicolumn{1}{c}{ Source } & SSQ & F ratio & df & P value \\
\hline & Between Subjects & & & \\
All Between & 0.0124 & 0.25 & 3,60 & 0.8626 \\
Treatment & 0.0022 & 0.13 & 1,60 & 0.7153 \\
Sex & 0.0029 & 0.17 & 1,60 & 0.6788 \\
Treatment-by-Sex & 0.0076 & 0.46 & 1,60 & 0.5022 \\
& & & & \\
All Within Interactions & Within Subjects & & & \\
Side & 0.0739 & 1.48 & 3,60 & 0.2294 \\
Side-by-Treatment & 0.0010 & 0.06 & 1,60 & 0.8080 \\
Side-by-Sex & 0.0304 & 1.82 & 1,60 & 0.1821 \\
Side-by-Treatment-by-Sex & 0.0542 & 3.25 & 1,60 & 0.0765 \\
& 0.0006 & 0.04 & 1,60 & 0.8444 \\
\hline
\end{tabular}

Table B-72. Results of mixed-model ANOVA testing for treatment (Edgewise with or without MARA) sex (male, female), and side (left, right TMJ) differences for Change in Sagittal EAM P L and Change in EAM P R.

\begin{tabular}{lcccc}
\hline \multicolumn{1}{c}{ Source } & SSQ & F ratio & df & P value \\
\hline & Between Subjects & & & \\
All Between & 0.0121 & 0.24 & 3,60 & 0.8672 \\
Treatment & 0.0012 & 0.07 & 1,60 & 0.7924 \\
Sex & 0.0115 & 0.69 & 1,60 & 0.4094 \\
Treatment-by-Sex & 0.0004 & 0.02 & 1,60 & 0.8809 \\
& & & & \\
All Within Interactions & Within Subjects & & & \\
Side & 0.0441 & 0.88 & 3,60 & 0.4560 \\
Side-by-Treatment & 0.1057 & 6.34 & 1,60 & 0.0145 \\
Side-by-Sex & 0.0227 & 1.36 & 1,60 & 0.2482 \\
Side-by-Treatment-by-Sex & 0.0070 & 0.42 & 1,60 & 0.5202 \\
& 0.0093 & 0.56 & 1,60 & 0.4590 \\
\hline
\end{tabular}


Table B-73. Results of mixed-model ANOVA testing for treatment (Edgewise with or without MARA) sex (male, female), and side (left, right TMJ) differences for Sagittal ATA L Initial and Sagittal ATA R Initial.

\begin{tabular}{lcrrr}
\hline \multicolumn{1}{c}{ Source } & SSQ & F ratio & df & P value \\
\hline & Between Subjects & & & \\
All Between & 0.4216 & 8.43 & 3,60 & $<0.0001$ \\
Treatment & 0.4170 & 25.02 & 1,60 & $<0.0001$ \\
Sex & 0.0012 & 0.07 & 1,60 & 0.7899 \\
Treatment-by-Sex & 0.0000 & 0.00 & 1,60 & 0.9898 \\
& & & & \\
All Within Interactions & Within Subjects & & & \\
Side & 0.0244 & 0.49 & 3,60 & 0.6917 \\
Side-by-Treatment & 0.0043 & 0.26 & 1,60 & 0.6117 \\
Side-by-Sex & 0.0105 & 0.63 & 1,60 & 0.4309 \\
Side-by-Treatment-by-Sex & 0.0162 & 0.97 & 1,60 & 0.3286 \\
& 0.0016 & 0.10 & 1,60 & 0.7561 \\
\hline
\end{tabular}

Table B-74. Results of mixed-model ANOVA testing for treatment (Edgewise with or without MA0052A) sex (male, female), and side (left, right TMJ) differences for Sagittal ATA L Final and Sagittal ATA R Final.

\begin{tabular}{lcccc}
\hline \multicolumn{1}{c}{ Source } & SSQ & F ratio & df & P value \\
\hline & Between Subjects & & & \\
All Between & 0.1483 & 2.97 & 3,60 & 0.0390 \\
Treatment & 0.1374 & 8.24 & 1,60 & 0.0056 \\
Sex & 0.0252 & 1.51 & 1,60 & 0.2233 \\
Treatment-by-Sex & 0.0002 & 0.01 & 1,60 & 0.9074 \\
& & & & \\
All Within Interactions & Within Subjects & & & \\
Side & 0.0215 & 0.43 & 3,60 & 0.7324 \\
Side-by-Treatment & 0.0107 & 0.64 & 1,60 & 0.4269 \\
Side-by-Sex & 0.0051 & 0.31 & 1,60 & 0.5804 \\
Side-by-Treatment-by-Sex & 0.0055 & 0.33 & 1,60 & 0.5685 \\
& 0.0129 & 0.77 & 1,60 & 0.3825 \\
\hline
\end{tabular}


Table B-75. Results of mixed-model ANOVA testing for treatment (Edgewise with or without MARA) sex (male, female), and side (left, right TMJ) differences for Change in Sagittal ATA $L$ and Change in Sagittal ATA R.

\begin{tabular}{lcrrr}
\hline \multicolumn{1}{c}{ Source } & SSQ & F ratio & df & P value \\
\hline & Between Subjects & & & \\
All Between & 0.3251 & 6.50 & 3,60 & 0.0007 \\
Treatment & 0.2283 & 13.70 & 1,60 & 0.0005 \\
Sex & 0.0534 & 3.20 & 1,60 & 0.0786 \\
Treatment-by-Sex & 0.0006 & 0.04 & 1,60 & 0.8481 \\
& & & & \\
All Within Interactions & Within Subjects & & & \\
Side & 0.0110 & 0.22 & 3,60 & 0.8816 \\
Side-by-Treatment & 0.0015 & 0.09 & 1,60 & 0.7669 \\
Side-by-Sex & 0.0013 & 0.08 & 1,60 & 0.7778 \\
Side-by-Treatment-by-Sex & 0.0038 & 0.23 & 1,60 & 0.6339 \\
& 0.0061 & 0.37 & 1,60 & 0.5467 \\
\hline
\end{tabular}

Table B-76. Results of mixed-model ANOVA testing for treatment (Edgewise with or without MARA) sex (male, female), and side (left, right TMJ) differences for Sagittal CHA L Initial and Sagittal CHA R Initial.

\begin{tabular}{lcccc}
\hline \multicolumn{1}{c}{ Source } & SSQ & F ratio & df & P value \\
\hline & Between Subjects & & & \\
All Between & 0.0755 & 1.51 & 3,60 & 0.2213 \\
Treatment & 0.0548 & 3.29 & 1,60 & 0.0748 \\
Sex & 0.0000 & 0.00 & 1,60 & 0.9665 \\
Treatment-by-Sex & 0.0200 & 1.20 & 1,60 & 0.2775 \\
& & & & \\
All Within Interactions & Within Subjects & & & \\
Side & 0.1278 & 2.56 & 3,60 & 0.0636 \\
Side-by-Treatment & 0.0049 & 0.30 & 1,60 & 0.5880 \\
Side-by-Sex & 0.1068 & 6.41 & 1,60 & 0.0140 \\
Side-by-Treatment-by-Sex & 0.0002 & 0.01 & 1,60 & 0.9221 \\
& 0.0165 & 0.99 & 1,60 & 0.3236 \\
\hline
\end{tabular}


Table B-77. Results of mixed-model ANOVA testing for treatment (Edgewise with or without MARA) sex (male, female), and side (left, right TMJ) differences for Sagittal CHA L Final and Sagittal CHA R Final.

\begin{tabular}{lcccc}
\hline \multicolumn{1}{c}{ Source } & SSQ & F ratio & df & P value \\
\hline & Between Subjects & & & \\
All Between & 0.1245 & 2.49 & 3,60 & 0.0689 \\
Treatment & 0.0501 & 3.00 & 1,60 & 0.0882 \\
Sex & 0.0003 & 0.02 & 1,60 & 0.9010 \\
Treatment-by-Sex & 0.0749 & 4.49 & 1,60 & 0.0382 \\
& & & & \\
All Within Interactions & Within Subjects & & & \\
Side & 0.0922 & 1.84 & 3,60 & 0.1490 \\
Side-by-Treatment & 0.0080 & 0.48 & 1,60 & 0.4916 \\
Side-by-Sex & 0.0660 & 3.96 & 1,60 & 0.0512 \\
Side-by-Treatment-by-Sex & 0.0107 & 0.64 & 1,60 & 0.4257 \\
& 0.0053 & 0.32 & 1,60 & 0.5749 \\
\hline
\end{tabular}

Table B-78. Results of mixed-model ANOVA testing for treatment (Edgewise with or without MARA) sex (male, female), and side (left, right TMJ) differences for Change in Sagittal CHA $L$ and Change in Sagittal CHA R.

\begin{tabular}{lcccc}
\hline \multicolumn{1}{c}{ Source } & SSQ & F ratio & df & P value \\
\hline & Between Subjects & & & \\
All Between & 0.0512 & 1.02 & 3,60 & 0.3887 \\
Treatment & 0.0048 & 0.29 & 1,60 & 0.5933 \\
Sex & 0.0002 & 0.01 & 1,60 & 0.9058 \\
Treatment-by-Sex & 0.0467 & 2.80 & 1,60 & 0.0995 \\
& & & & \\
All Within Interactions & Within Subjects & & & \\
Side & 0.0084 & 0.17 & 3,60 & 0.9177 \\
Side-by-Treatment & 0.0175 & 1.05 & 1,60 & 0.3091 \\
Side-by-Sex & 0.0011 & 0.06 & 1,60 & 0.8024 \\
Side-by-Treatment-by-Sex & 0.0064 & 0.38 & 1,60 & 0.5382 \\
& 0.0014 & 0.08 & 1,60 & 0.7743 \\
\hline
\end{tabular}


Table B-79. Results of mixed-model ANOVA testing for treatment (Edgewise with or without MARA) sex (male, female), and side (left, right TMJ) differences for Sagittal ACP L Initial and Sagittal ACP R Initial.

\begin{tabular}{lcccc}
\hline \multicolumn{1}{c}{ Source } & SSQ & F ratio & df & P value \\
\hline & Between Subjects & & & \\
All Between & 0.1822 & 3.64 & 3,60 & 0.0175 \\
Treatment & 0.1480 & 8.88 & 1,60 & 0.0042 \\
Sex & 0.0171 & 1.03 & 1,60 & 0.3148 \\
Treatment-by-Sex & 0.0270 & 1.62 & 1,60 & 0.2081 \\
& & & & \\
All Within Interactions & Within Subjects & & & \\
Side & 0.0146 & 0.29 & 3,60 & 0.8315 \\
Side-by-Treatment & 0.0000 & 0.00 & 1,60 & 0.9741 \\
Side-by-Sex & 0.0049 & 0.29 & 1,60 & 0.5911 \\
Side-by-Treatment-by-Sex & 0.0074 & 0.45 & 1,60 & 0.5067 \\
& 0.0001 & 0.00 & 1,60 & 0.9524 \\
\hline
\end{tabular}

Table B-80. Results of mixed-model ANOVA testing for treatment (Edgewise with or without MARA) sex (male, female), and side (left, right TMJ) differences for Sagittal ACP L Final and Sagittal ACP R Final.

\begin{tabular}{lcrcc}
\hline \multicolumn{1}{c}{ Source } & SSQ & F ratio & df & P value \\
\hline & Between Subjects & & & \\
All Between & 0.2929 & 5.86 & 3,60 & 0.0014 \\
Treatment & 0.2346 & 14.08 & 1,60 & 0.0004 \\
Sex & 0.0958 & 5.75 & 1,60 & 0.0197 \\
Treatment-by-Sex & 0.0013 & 0.08 & 1,60 & 0.7796 \\
& & & & \\
All Within Interactions & Within Subjects & & & \\
Side & 0.0217 & 0.43 & 3,60 & 0.7290 \\
Side-by-Treatment & 0.0005 & 0.03 & 1,60 & 0.8611 \\
Side-by-Sex & 0.0003 & 0.02 & 1,60 & 0.9001 \\
Side-by-Treatment-by-Sex & 0.0002 & 0.01 & 1,60 & 0.9048 \\
& 0.0211 & 1.27 & 1,60 & 0.2650 \\
\hline
\end{tabular}


Table B-81. Results of mixed-model ANOVA testing for treatment (Edgewise with or without MARA) sex (male, female), and side (left, right TMJ) differences for Change in Sagittal ACP L and Change in Sagittal ACP R.

\begin{tabular}{lcccc}
\hline \multicolumn{1}{c}{ Source } & SSQ & F ratio & df & P value \\
\hline & Between Subjects & & & \\
All Between & 0.1336 & 2.67 & 3,60 & 0.0555 \\
Treatment & 0.0265 & 1.59 & 1,60 & 0.2123 \\
Sex & 0.0821 & 4.92 & 1,60 & 0.0303 \\
Treatment-by-Sex & 0.0411 & 2.47 & 1,60 & 0.1214 \\
& & & & \\
All Within Interactions & Within Subjects & & & \\
Side & 0.0354 & 0.71 & 3,60 & 0.5507 \\
Side-by-Treatment & 0.0007 & 0.04 & 1,60 & 0.8335 \\
Side-by-Sex & 0.0059 & 0.35 & 1,60 & 0.5548 \\
Side-by-Treatment-by-Sex & 0.0033 & 0.20 & 1,60 & 0.6568 \\
& 0.0250 & 1.50 & 1,60 & 0.2254 \\
\hline
\end{tabular}

Table B-82. Results of mixed-model ANOVA testing for treatment (Edgewise with or without MARA) sex (male, female), and side (left, right TMJ) differences for Sagittal AAE L Initial and Sagittal AAE R Initial.

\begin{tabular}{lcccc}
\hline \multicolumn{1}{c}{ Source } & SSQ & F ratio & df & P value \\
\hline & Between Subjects & & & \\
All Between & 0.0962 & 1.92 & 3,60 & 0.1352 \\
Treatment & 0.0248 & 1.49 & 1,60 & 0.2270 \\
Sex & 0.0481 & 2.89 & 1,60 & 0.0946 \\
Treatment-by-Sex & 0.0117 & 0.70 & 1,60 & 0.4057 \\
& & & & \\
All Within Interactions & Within Subjects & & & \\
Side & 0.0718 & 1.44 & 3,60 & 0.2413 \\
Side-by-Treatment & 0.0583 & 3.50 & 1,60 & 0.0664 \\
Side-by-Sex & 0.0324 & 1.94 & 1,60 & 0.1686 \\
Side-by-Treatment-by-Sex & 0.0029 & 0.18 & 1,60 & 0.6755 \\
& 0.0327 & 1.96 & 1,60 & 0.1664 \\
\hline
\end{tabular}


Table B-83. Results of mixed-model ANOVA testing for treatment (Edgewise with or without MARA) sex (male, female), and side (left, right TMJ) differences for Sagittal AAE L Final and Sagittal AAE R Final.

\begin{tabular}{lcccc}
\hline \multicolumn{1}{c}{ Source } & SSQ & F ratio & df & P value \\
\hline & Between Subjects & & & \\
All Between & 0.0320 & 0.64 & 3,60 & 0.5927 \\
Treatment & 0.0040 & 0.24 & 1,60 & 0.6263 \\
Sex & 0.0223 & 1.34 & 1,60 & 0.2521 \\
Treatment-by-Sex & 0.0024 & 0.15 & 1,60 & 0.7047 \\
& & & & \\
All Within Interactions & Within Subjects & & & \\
Side & 0.0436 & 0.87 & 3,60 & 0.4605 \\
Side-by-Treatment & 0.0088 & 0.53 & 1,60 & 0.4704 \\
Side-by-Sex & 0.0037 & 0.22 & 1,60 & 0.6386 \\
Side-by-Treatment-by-Sex & 0.0010 & 0.06 & 1,60 & 0.8038 \\
& 0.0388 & 2.33 & 1,60 & 0.1323 \\
\hline
\end{tabular}

Table B-84. Results of mixed-model ANOVA testing for treatment (Edgewise with or without MARA) sex (male, female), and side (left, right TMJ) differences for Change in Sagittal AAE $L$ and Change in Sagittal AAE R.

\begin{tabular}{lcccc}
\hline \multicolumn{1}{c}{ Source } & SSQ & F ratio & df & P value \\
\hline & Between Subjects & & & \\
All Between & 0.0255 & 0.51 & 3,60 & 0.6762 \\
Treatment & 0.0143 & 0.86 & 1,60 & 0.3573 \\
Sex & 0.0035 & 0.21 & 1,60 & 0.6488 \\
Treatment-by-Sex & 0.0052 & 0.31 & 1,60 & 0.5794 \\
& & & & \\
All Within Interactions & Within Subjects & & & \\
Side & 0.0269 & 0.54 & 3,60 & 0.6577 \\
Side-by-Treatment & 0.0143 & 0.86 & 1,60 & 0.3581 \\
Side-by-Sex & 0.0100 & 0.60 & 1,60 & 0.4409 \\
Side-by-Treatment-by-Sex & 0.0086 & 0.52 & 1,60 & 0.4751 \\
& 0.0045 & 0.27 & 1,60 & 0.6055 \\
\hline
\end{tabular}


Table B-85. Results of mixed-model ANOVA testing for treatment (Edgewise with or without MARA) sex (male, female), and side (left, right TMJ) differences for Sagittal AGF L Initial and Sagittal AGF R Initial.

\begin{tabular}{lcccc}
\hline \multicolumn{1}{c}{ Source } & SSQ & F ratio & df & P value \\
\hline & Between Subjects & & & \\
All Between & 0.0902 & 1.80 & 3,60 & 0.1561 \\
Treatment & 0.0858 & 5.15 & 1,60 & 0.0268 \\
Sex & 0.0001 & 0.01 & 1,60 & 0.9426 \\
Treatment-by-Sex & 0.0013 & 0.08 & 1,60 & 0.7812 \\
& & & & \\
All Within Interactions & Within Subjects & & & \\
Side & 0.1659 & 3.32 & 3,60 & 0.0258 \\
Side-by-Treatment & 0.1580 & 9.48 & 1,60 & 0.0031 \\
Side-by-Sex & 0.1297 & 7.78 & 1,60 & 0.0071 \\
Side-by-Treatment-by-Sex & 0.0060 & 0.36 & 1,60 & 0.5520 \\
& 0.0177 & 1.06 & 1,60 & 0.3073 \\
\hline
\end{tabular}

Table B-86. Results of mixed-model ANOVA testing for treatment (Edgewise with or without MARA) sex (male, female), and side (left, right TMJ) differences for Sagittal AGF L Final and Sagittal AGF R Final.

\begin{tabular}{lcccc}
\hline \multicolumn{1}{c}{ Source } & SSQ & F ratio & df & P value \\
\hline & Between Subjects & & & \\
All Between & 0.0115 & 0.23 & 3,60 & 0.8757 \\
Treatment & 0.0027 & 0.16 & 1,60 & 0.6874 \\
Sex & 0.0000 & 0.00 & 1,60 & 0.9902 \\
Treatment-by-Sex & 0.0087 & 0.52 & 1,60 & 0.4718 \\
& & & & \\
All Within Interactions & Within Subjects & & & \\
Side & & & & \\
Side-by-Treatment & 0.1402 & 8.41 & 3,60 & 0.0052 \\
Side-by-Sex & 0.0305 & 1.83 & 1,60 & 0.1813 \\
Side-by-Treatment-by-Sex & 0.0143 & 0.86 & 1,60 & 0.3579 \\
& 0.0060 & 0.36 & 1,60 & 0.5493 \\
\hline
\end{tabular}


Table B-87. Results of mixed-model ANOVA testing for treatment (Edgewise with or without MARA) sex (male, female), and side (left, right TMJ) differences for Change in Sagittal AGF L and Change in Sagittal AGF R.

\begin{tabular}{lcccc}
\hline \multicolumn{1}{c}{ Source } & SSQ & F ratio & df & P value \\
\hline & Between Subjects & & & \\
All Between & 0.1710 & 3.42 & 3,60 & 0.0228 \\
Treatment & 0.1539 & 9.24 & 1,60 & 0.0035 \\
Sex & 0.0003 & 0.02 & 1,60 & 0.8890 \\
Treatment-by-Sex & 0.0103 & 0.62 & 1,60 & 0.4352 \\
& & & & \\
All Within Interactions & Within Subjects & & & \\
Side & 0.0263 & 0.53 & 3,60 & 0.6668 \\
Side-by-Treatment & 0.0010 & 0.06 & 1,60 & 0.8044 \\
Side-by-Sex & 0.0235 & 1.41 & 1,60 & 0.2396 \\
Side-by-Treatment-by-Sex & 0.0034 & 0.20 & 1,60 & 0.6539 \\
& 0.0018 & 0.11 & 1,60 & 0.7468 \\
\hline
\end{tabular}

Table B-88. Results of mixed-model ANOVA testing for treatment (Edgewise with or without MARA) sex (male, female), and side (left, right TMJ) differences for Coronal MJS L Initial and Coronal MJS R Initial.

\begin{tabular}{lcccc}
\hline \multicolumn{1}{c}{ Source } & SSQ & F ratio & df & P value \\
\hline & Between Subjects & & & \\
All Between & 0.0074 & 0.15 & 3,60 & 0.9301 \\
Treatment & 0.0017 & 0.10 & 1,60 & 0.7508 \\
Sex & 0.0055 & 0.33 & 1,60 & 0.5665 \\
Treatment-by-Sex & 0.0012 & 0.07 & 1,60 & 0.7912 \\
& & & & \\
All Within Interactions & Within Subjects & & & \\
Side & 0.0035 & 0.07 & 3,60 & 0.9755 \\
Side-by-Treatment & 0.0067 & 0.40 & 1,60 & 0.5281 \\
Side-by-Sex & 0.0002 & 0.01 & 1,60 & 0.9059 \\
Side-by-Treatment-by-Sex & 0.0029 & 0.18 & 1,60 & 0.6761 \\
& 0.0000 & 0.00 & 1,60 & 0.9970 \\
\hline
\end{tabular}


Table B-89. Results of mixed-model ANOVA testing for treatment (Edgewise with or without MARA) sex (male, female), and side (left, right TMJ) differences for Coronal MJS L Final and Coronal MJS R Final.

\begin{tabular}{lcccc}
\hline \multicolumn{1}{c}{ Source } & SSQ & F ratio & df & P value \\
\hline & Between Subjects & & & \\
All Between & 0.0183 & 0.37 & 3,60 & 0.7771 \\
Treatment & 0.0000 & 0.00 & 1,60 & 0.9805 \\
Sex & 0.0146 & 0.88 & 1,60 & 0.3533 \\
Treatment-by-Sex & 0.0028 & 0.17 & 1,60 & 0.6816 \\
& & & & \\
All Within Interactions & Within Subjects & & & \\
Side & 0.1782 & 3.56 & 3,60 & 0.0193 \\
Side-by-Treatment & 0.0684 & 4.10 & 1,60 & 0.0472 \\
Side-by-Sex & 0.1681 & 10.08 & 1,60 & 0.0024 \\
Side-by-Treatment-by-Sex & 0.0001 & 0.01 & 1,60 & 0.9435 \\
& 0.0066 & 0.40 & 1,60 & 0.5314 \\
\hline
\end{tabular}

Table B-90. Results of mixed-model ANOVA testing for treatment (Edgewise with or without MARA) sex (male, female), and side (left, right TMJ) differences for Change in Coronal MJS L and Change in Coronal MJS R.

\begin{tabular}{lcccc}
\hline \multicolumn{1}{c}{ Source } & SSQ & F ratio & df & P value \\
\hline & Between Subjects & & & \\
All Between & 0.0432 & 0.86 & 3,60 & 0.4647 \\
Treatment & 0.0013 & 0.08 & 1,60 & 0.7822 \\
Sex & 0.0421 & 2.53 & 1,60 & 0.1172 \\
Treatment-by-Sex & 0.0007 & 0.04 & 1,60 & 0.8393 \\
& & & & \\
All Within Interactions & Within Subjects & & & \\
Side & 0.1575 & 3.15 & 3,60 & 0.0314 \\
Side-by-Treatment & 0.0229 & 1.38 & 1,60 & 0.2456 \\
Side-by-Sex & 0.1414 & 8.49 & 1,60 & 0.0050 \\
Side-by-Treatment-by-Sex & 0.0020 & 0.12 & 1,60 & 0.7331 \\
\hline
\end{tabular}


Table B-91. Results of mixed-model ANOVA testing for treatment (Edgewise with or without MARA) sex (male, female), and side (left, right TMJ) differences for Coronal SJS L Initial and Coronal SJS R Initial.

\begin{tabular}{lcccc}
\hline \multicolumn{1}{c}{ Source } & SSQ & F ratio & df & P value \\
\hline & Between Subjects & & & \\
All Between & 0.0042 & 0.08 & 3,60 & 0.9690 \\
Treatment & 0.0025 & 0.15 & 1,60 & 0.7022 \\
Sex & 0.0004 & 0.02 & 1,60 & 0.8808 \\
Treatment-by-Sex & 0.0016 & 0.10 & 1,60 & 0.7559 \\
& & & & \\
All Within Interactions & Within Subjects & & & \\
Side & 0.0857 & 1.71 & 3,60 & 0.1736 \\
Side-by-Treatment & 0.0015 & 0.09 & 1,60 & 0.7672 \\
Side-by-Sex & 0.0805 & 4.83 & 1,60 & 0.0318 \\
Side-by-Treatment-by-Sex & 0.0001 & 0.00 & 1,60 & 0.9448 \\
& 0.0024 & 0.14 & 1,60 & 0.7081 \\
\hline
\end{tabular}

Table B-92. Results of mixed-model ANOVA testing for treatment (Edgewise with or without MARA) sex (male, female), and side (left, right TMJ) differences for Coronal SJS L F and Coronal SJS R Final.

\begin{tabular}{lcccc}
\hline \multicolumn{1}{c}{ Source } & SSQ & F ratio & df & P value \\
\hline & Between Subjects & & & \\
All Between & 0.0541 & 1.08 & 3,60 & 0.3639 \\
Treatment & 0.0273 & 1.64 & 1,60 & 0.2057 \\
Sex & 0.0144 & 0.87 & 1,60 & 0.3560 \\
Treatment-by-Sex & 0.0164 & 0.99 & 1,60 & 0.3246 \\
& & & & \\
All Within Interactions & Within Subjects & & & \\
Side & 0.0471 & 0.94 & 3,60 & 0.4257 \\
Side-by-Treatment & 0.0183 & 1.10 & 1,60 & 0.2989 \\
Side-by-Sex & 0.0046 & 0.28 & 1,60 & 0.6011 \\
Side-by-Treatment-by-Sex & 0.0061 & 0.37 & 1,60 & 0.5470 \\
& 0.0336 & 2.02 & 1,60 & 0.1607 \\
\hline
\end{tabular}


Table B-93. Results of mixed-model ANOVA testing for treatment (Edgewise with or without MARA) sex (male, female), and side (left, right TMJ) differences for Change in Coronal SJS L and Change in Coronal SJS R.

\begin{tabular}{lcccc}
\hline \multicolumn{1}{c}{ Source } & SSQ & F ratio & df & P value \\
\hline & Between Subjects & & & \\
All Between & 0.0441 & 0.88 & 3,60 & 0.4556 \\
Treatment & 0.0125 & 0.75 & 1,60 & 0.3903 \\
Sex & 0.0092 & 0.55 & 1,60 & 0.4613 \\
Treatment-by-Sex & 0.0242 & 1.45 & 1,60 & 0.2331 \\
& & & & \\
All Within Interactions & Within Subjects & & & \\
Side & 0.0953 & 1.91 & 3,60 & 0.1381 \\
Side-by-Treatment & 0.0164 & 0.99 & 1,60 & 0.3246 \\
Side-by-Sex & 0.0548 & 3.29 & 1,60 & 0.0747 \\
Side-by-Treatment-by-Sex & 0.0043 & 0.26 & 1,60 & 0.6130 \\
& 0.0294 & 1.77 & 1,60 & 0.1889 \\
\hline
\end{tabular}

Table B-94. Results of mixed-model ANOVA testing for treatment (Edgewise with or without MARA) sex (male, female), and side (left, right TMJ) differences for Coronal LJS L Initial and Coronal LJS R Initial.

\begin{tabular}{lcccc}
\hline \multicolumn{1}{c}{ Source } & SSQ & F ratio & df & P value \\
\hline & Between Subjects & & & \\
All Between & 0.0688 & 1.38 & 3,60 & 0.2590 \\
Treatment & 0.0604 & 3.63 & 1,60 & 0.0617 \\
Sex & 0.0027 & 0.16 & 1,60 & 0.6910 \\
Treatment-by-Sex & 0.0000 & 0.00 & 1,60 & 0.9747 \\
& & & & \\
All Within Interactions & Within Subjects & & & \\
Side & 0.0207 & 0.41 & 3,60 & 0.7440 \\
Side-by-Treatment & 0.0189 & 1.13 & 1,60 & 0.2912 \\
Side-by-Sex & 0.0125 & 0.75 & 1,60 & 0.3899 \\
Side-by-Treatment-by-Sex & 0.0029 & 0.17 & 1,60 & 0.6787 \\
& 0.0064 & 0.39 & 1,60 & 0.5365 \\
\hline
\end{tabular}


Table B-95. Results of mixed-model ANOVA testing for treatment (Edgewise with or without MARA) sex (male, female), and side (left, right TMJ) differences for Coronal LJS L Final and Coronal LJS R Final.

\begin{tabular}{lcccc}
\hline \multicolumn{1}{c}{ Source } & SSQ & F ratio & df & P value \\
\hline & Between Subjects & & & \\
All Between & 0.0448 & 0.90 & 3,60 & 0.4482 \\
Treatment & 0.0332 & 1.99 & 1,60 & 0.1633 \\
Sex & 0.0181 & 1.09 & 1,60 & 0.3014 \\
Treatment-by-Sex & 0.0003 & 0.02 & 1,60 & 0.9011 \\
& & & & \\
All Within Interactions & Within Subjects & & & \\
Side & 0.0847 & 1.69 & 3,60 & 0.1778 \\
Side-by-Treatment & 0.0002 & 0.01 & 1,60 & 0.9186 \\
Side-by-Sex & 0.0013 & 0.08 & 1,60 & 0.7803 \\
Side-by-Treatment-by-Sex & 0.0171 & 1.02 & 1,60 & 0.3156 \\
& 0.0623 & 3.74 & 1,60 & 0.0580 \\
\hline
\end{tabular}

Table B-96. Results of mixed-model ANOVA testing for treatment (Edgewise with or without MARA) sex (male, female), and side (left, right TMJ) differences for Change in Coronal LJS L and Change in Coronal LJS R.

\begin{tabular}{lcccc}
\hline \multicolumn{1}{c}{ Source } & SSQ & F ratio & df & P value \\
\hline & Between Subjects & & & \\
All Between & 0.0468 & 0.94 & 3,60 & 0.4292 \\
Treatment & 0.0020 & 0.12 & 1,60 & 0.7282 \\
Sex & 0.0405 & 2.43 & 1,60 & 0.1242 \\
Treatment-by-Sex & 0.0005 & 0.03 & 1,60 & 0.8652 \\
& & & & \\
All Within Interactions & Within Subjects & & & \\
Side & 0.0480 & 0.96 & 3,60 & 0.4174 \\
Side-by-Treatment & 0.0223 & 1.34 & 1,60 & 0.2516 \\
Side-by-Sex & 0.0207 & 1.24 & 1,60 & 0.2691 \\
Side-by-Treatment-by-Sex & 0.0038 & 0.23 & 1,60 & 0.6348 \\
& 0.0196 & 1.18 & 1,60 & 0.2820 \\
\hline
\end{tabular}


APPENDIX C. LEAST-SQUARES MEANS FOR THE VARIABLES, PARTITIONED BY TREATMENT, SEX, AND TREATMENT-BY-SEX

Table C-1. Descriptive statistics (least-square means) of the sample partitioned by treatment and sex for the variable Axial ML L Initial and Axial ML R Initial.

\begin{tabular}{|c|c|c|c|c|c|}
\hline \multirow[b]{3}{*}{ Group } & \multicolumn{2}{|c|}{ Treatment-by-Sex } & & \multicolumn{2}{|c|}{ Sexes Combined } \\
\hline & Left & Right & & Left & Right \\
\hline & Initial & Initial & & Initial & Initial \\
\hline Edgewise, Female & 17.233 & 17.478 & Edgewise & 17.471 & 17.685 \\
\hline Edgewise, Male & 17.708 & 17.892 & MARA+Edgewise & 16.949 & 16.958 \\
\hline MARA+Edgewise, Female & 16.193 & 16.057 & & & \\
\hline MARA+Edgewise, Male & 17.705 & 17.858 & & & \\
\hline
\end{tabular}

Table C-2. Descriptive statistics (least-square means) of the sample partitioned by treatment and sex for the variable Axial ML L Final and Axial ML R Final.

\begin{tabular}{lccccc}
\hline & \multicolumn{2}{c}{ Treatment-by-Sex } & & \multicolumn{2}{c}{ Sexes Combined } \\
\multicolumn{1}{c}{ Group } & $\begin{array}{c}\text { Left } \\
\text { Final }\end{array}$ & $\begin{array}{c}\text { Right } \\
\text { Final }\end{array}$ & Final & $\begin{array}{c}\text { Right } \\
\text { Final }\end{array}$ \\
\hline Edgewise, Female & 17.983 & 18.406 & Edgewise & 18.465 & 18.449 \\
Edgewise, Male & 18.946 & 18.492 & MARA+Edgewise & 17.567 & 17.790 \\
MARA+Edgewise, Female & 16.493 & 16.743 & & \\
MARA+Edgewise, Male & 18.642 & 18.837 & & \\
\hline
\end{tabular}


Table C-3. Descriptive statistics (least-square means) of the sample partitioned by treatment and sex for the variable Change in Axial ML $L$ and Change in Axial ML R.

\begin{tabular}{|c|c|c|c|c|c|}
\hline \multirow[b]{2}{*}{ Group } & \multicolumn{2}{|c|}{ Treatment-by-Sex } & & \multicolumn{2}{|c|}{$\underline{\text { Sexes Combined }}$} \\
\hline & $\begin{array}{c}\text { Left } \\
\text { Change }\end{array}$ & $\begin{array}{c}\text { Right } \\
\text { Change }\end{array}$ & & $\begin{array}{c}\text { Left } \\
\text { Change }\end{array}$ & $\begin{array}{c}\text { Right } \\
\text { Change }\end{array}$ \\
\hline Edgewise, Female & 0.750 & 0.928 & Edgewise & 0.994 & 0.764 \\
\hline Edgewise, Male & 1.238 & 0.600 & MARA+Edgewise & 0.618 & 0.832 \\
\hline MARA+Edgewise, Female & 0.300 & 0.686 & & & \\
\hline MARA+Edgewise, Male & 0.937 & 0.979 & & & \\
\hline
\end{tabular}

Table C-4. Descriptive statistics (least-square means) of the sample partitioned by treatment and sex for the variable Axial AP L Initial and Axial AP R Initial.

\begin{tabular}{|c|c|c|c|c|c|}
\hline \multirow[b]{2}{*}{ Group } & \multicolumn{2}{|c|}{ Treatment-by-Sex } & & \multicolumn{2}{|c|}{ Sexes Combined } \\
\hline & $\begin{array}{c}\text { Left } \\
\text { Initial }\end{array}$ & $\begin{array}{l}\text { Right } \\
\text { Initial }\end{array}$ & & $\begin{array}{c}\text { Left } \\
\text { Initial }\end{array}$ & $\begin{array}{l}\text { Right } \\
\text { Initial }\end{array}$ \\
\hline Edgewise, Female & 6.278 & 6.472 & Edgewise & 6.750 & 6.825 \\
\hline Edgewise, Male & 7.223 & 7.177 & MARA+Edgewise & 7.023 & 7.075 \\
\hline MARA+Edgewise, Female & 6.979 & 6.707 & & & \\
\hline MARA+Edgewise, Male & 7.068 & 7.442 & & & \\
\hline
\end{tabular}


Table C-5. Descriptive statistics (least-square means) of the sample partitioned by treatment and sex for the variable Axial AP L Final and Axial AP R Final.

\begin{tabular}{lccccc}
\hline & \multicolumn{2}{c}{ Treatment-by-Sex } & & \multicolumn{2}{c}{ Sexes Combined } \\
\multicolumn{1}{c}{ Left } & Right & & Right \\
Left & Final & Final & & Final & Final \\
\hline Edgewise, Female & 6.256 & 6.472 & Edgewise & 6.755 & 6.778 \\
Edgewise, Male & 7.254 & 7.085 & MARA+Edgewise & 6.715 & 6.673 \\
MARA+Edgewise, Female & 6.493 & 6.393 & & \\
MARA+Edgewise, Male & 6.937 & 6.953 & & \\
\hline
\end{tabular}

Table C-6. Descriptive statistics (least-square means) of the sample partitioned by treatment and sex for the variable Change in Axial AP $L$ and Change in Axial AP R.

\begin{tabular}{|c|c|c|c|c|c|}
\hline \multirow[b]{2}{*}{ Group } & \multicolumn{2}{|c|}{ Treatment-by-Sex } & & \multicolumn{2}{|c|}{ Sexes Combined } \\
\hline & $\begin{array}{c}\text { Left } \\
\text { Change }\end{array}$ & $\begin{array}{c}\text { Right } \\
\text { Change }\end{array}$ & & $\begin{array}{c}\text { Left } \\
\text { Change }\end{array}$ & $\begin{array}{l}\text { Right } \\
\text { Change }\end{array}$ \\
\hline Edgewise, Female & -0.022 & 0.000 & Edgewise & 0.004 & -0.046 \\
\hline Edgewise, Male & 0.031 & -0.092 & MARA+Edgewise & -0.309 & -0.402 \\
\hline MARA+Edgewise, Female & -0.486 & -0.314 & & & \\
\hline MARA+Edgewise, Male & -0.132 & -0.489 & & & \\
\hline
\end{tabular}


Table C-7. Descriptive statistics (least-square means) of the sample partitioned by treatment and sex for the variable Axial MLCP L Initial and Axial MLCP R Initial.

\begin{tabular}{|c|c|c|c|c|c|}
\hline \multirow[b]{2}{*}{ Group } & \multicolumn{2}{|c|}{ Treatment-by-Sex } & & \multicolumn{2}{|c|}{ Sexes Combined } \\
\hline & $\begin{array}{l}\text { Left } \\
\text { Initial }\end{array}$ & $\begin{array}{l}\text { Right } \\
\text { Initial }\end{array}$ & & $\begin{array}{l}\text { Left } \\
\text { Initial }\end{array}$ & $\begin{array}{l}\text { Right } \\
\text { Initial }\end{array}$ \\
\hline Edgewise, Female & 46.072 & 46.256 & Edgewise & 46.182 & 46.693 \\
\hline Edgewise, Male & 46.292 & 47.131 & MARA+Edgewise & 46.055 & 46.007 \\
\hline MARA+Edgewise, Female & 45.436 & 45.050 & & & \\
\hline MARA+Edgewise, Male & 46.674 & 46.963 & & & \\
\hline
\end{tabular}

Table C-8. Descriptive statistics (least-square means) of the sample partitioned by treatment and sex for the variable Axial MLCP L Final and Axial MLCP R Final.

\begin{tabular}{|c|c|c|c|c|c|}
\hline \multirow{3}{*}{ Group } & \multicolumn{2}{|c|}{ Treatment-by-Sex } & & \multicolumn{2}{|c|}{ Sexes Combined } \\
\hline & Left & Right & & Left & Right \\
\hline & Final & Final & & Final & Final \\
\hline Edgewise, Female & 47.333 & 47.333 & Edgewise & 47.486 & 48.055 \\
\hline Edgewise, Male & 47.638 & 48.777 & MARA+Edgewise & 47.527 & 47.472 \\
\hline MARA+Edgewise, Female & 46.786 & 46.464 & & & \\
\hline MARA+Edgewise, Male & 48.268 & 48.479 & & & \\
\hline
\end{tabular}


Table C-9. Descriptive statistics (least-square means) of the sample partitioned by treatment and sex for the variable Change in Axial MLCP $L$ and Change in Axial MLCP R.

\begin{tabular}{|c|c|c|c|c|c|}
\hline \multirow[b]{2}{*}{ Group } & \multicolumn{2}{|c|}{ Treatment-by-Sex } & & \multicolumn{2}{|c|}{$\underline{\text { Sexes Combined }}$} \\
\hline & $\begin{array}{c}\text { Left } \\
\text { Change }\end{array}$ & $\begin{array}{c}\text { Right } \\
\text { Change }\end{array}$ & & $\begin{array}{c}\text { Left } \\
\text { Change }\end{array}$ & $\begin{array}{c}\text { Right } \\
\text { Change }\end{array}$ \\
\hline Edgewise, Female & 1.261 & 1.078 & Edgewise & 1.304 & 1.362 \\
\hline Edgewise, Male & 1.346 & 1.646 & MARA+Edgewise & 1.472 & 1.465 \\
\hline MARA+Edgewise, Female & 1.350 & 1.414 & & & \\
\hline MARA+Edgewise, Male & 1.595 & 1.516 & & & \\
\hline
\end{tabular}

Table C-10. Descriptive statistics (least-square means) of the sample partitioned by treatment and sex for the variable Axial CA L Initial and Axial CA R Initial.

\begin{tabular}{|c|c|c|c|c|c|}
\hline \multirow[b]{3}{*}{ Group } & \multicolumn{2}{|c|}{ Treatment-by-Sex } & & \multicolumn{2}{|c|}{ Sexes Combined } \\
\hline & Left & Right & & Left & Right \\
\hline & Initial & Initial & & Initial & Initial \\
\hline Edgewise, Female & 63.528 & 62.833 & Edgewise & 63.387 & 63.247 \\
\hline Edgewise, Male & 63.246 & 63.662 & MARA+Edgewise & 61.676 & 61.779 \\
\hline MARA+Edgewise, Female & 61.814 & 60.100 & & & \\
\hline MARA+Edgewise, Male & 61.537 & 63.458 & & & \\
\hline
\end{tabular}


Table C-11. Descriptive statistics (least-square means) of the sample partitioned by treatment and sex for the variable Axial CA L Final and Axial CA R Final.

\begin{tabular}{lccccc}
\hline & \multicolumn{2}{c}{ Treatment-by-Sex } & & \multicolumn{2}{c}{ Sexes Combined } \\
\cline { 2 - 5 } \multicolumn{1}{c}{ Group } & Left & Right & & Right \\
Linal & Final & & Final & Final \\
\hline Edgewise, Female & 64.294 & 63.528 & Edgewise & 64.478 & 63.375 \\
Edgewise, Male & 64.662 & 63.223 & MARA+Edgewise & 62.754 & 62.270 \\
MARA+Edgewise, Female & 61.750 & 59.871 & & \\
MARA+Edgewise, Male & 63.758 & 64.668 & & & \\
\hline
\end{tabular}

Table C-12. Descriptive statistics (least-square means) of the sample partitioned by treatment and sex for the variable change in Axial CA $L$ and change in Axial CA R.

\begin{tabular}{|c|c|c|c|c|c|}
\hline \multirow[b]{2}{*}{ Group } & \multicolumn{2}{|c|}{ Treatment-by-Sex } & & \multicolumn{2}{|c|}{ Sexes Combined } \\
\hline & $\begin{array}{c}\text { Left } \\
\text { Change }\end{array}$ & $\begin{array}{l}\text { Right } \\
\text { Change }\end{array}$ & & $\begin{array}{c}\text { Left } \\
\text { Change }\end{array}$ & $\begin{array}{l}\text { Right } \\
\text { Change }\end{array}$ \\
\hline Edgewise-Alone, Female & 0.767 & 0.694 & Edgewise-Alone & 1.091 & 0.128 \\
\hline Edgewise-Alone, Male & 1.415 & -0.438 & MARA+Edgewise & 1.078 & 0.491 \\
\hline MARA+Edgewise, Female & -0.064 & -0.229 & & & \\
\hline MARA+Edgewise, Male & 2.221 & 1.211 & & & \\
\hline
\end{tabular}


Table C-13. Descriptive statistics (least-square means) of the sample partitioned by treatment and sex for the variable Axial LACP L Initial and Axial LACP R Initial.

\begin{tabular}{|c|c|c|c|c|c|}
\hline \multirow[b]{3}{*}{ Group } & \multicolumn{2}{|c|}{ Treatment-by-Sex } & & \multicolumn{2}{|c|}{$\underline{\text { Sexes Combined }}$} \\
\hline & Left & Right & & Left & Right \\
\hline & Initial & Initial & & Initial & Initial \\
\hline Edgewise, Female & 97.178 & 101.217 & Edgewise & 101.297 & 104.097 \\
\hline Edgewise, Male & 105.415 & 106.977 & MARA+Edgewise & 100.231 & 102.859 \\
\hline MARA+Edgewise, Female & 91.936 & 92.071 & & & \\
\hline MARA+Edgewise, Male & 108.526 & 113.647 & & & \\
\hline
\end{tabular}

Table C-14. Descriptive statistics (least-square means) of the sample partitioned by treatment and sex for the variable Axial LACP L Final and Axial LACP R Final.

\begin{tabular}{|c|c|c|c|c|c|}
\hline \multirow[b]{3}{*}{ Group } & \multicolumn{2}{|c|}{ Treatment-by-Sex } & & \multicolumn{2}{|c|}{$\underline{\text { Sexes Combined }}$} \\
\hline & Left & Right & & Left & Right \\
\hline & Final & Final & & Final & Final \\
\hline Edgewise, Female & 102.250 & 104.406 & Edgewise & 108.113 & 107.260 \\
\hline Edgewise, Male & 113.977 & 110.115 & MARA+Edgewise & 103.695 & 107.899 \\
\hline MARA+Edgewise, Female & 92.279 & 94.850 & & & \\
\hline MARA+Edgewise, Male & 115.111 & 120.947 & & & \\
\hline
\end{tabular}


Table C-15. Descriptive statistics (least-square means) of the sample partitioned by treatment and sex for the variable Change in Axial LACP $L$ and Change in Axial LACP R.

\begin{tabular}{|c|c|c|c|c|c|}
\hline \multirow[b]{2}{*}{ Group } & \multicolumn{2}{|c|}{ Treatment-by-Sex } & & \multicolumn{2}{|c|}{$\underline{\text { Sexes Combined }}$} \\
\hline & $\begin{array}{c}\text { Left } \\
\text { Change }\end{array}$ & $\begin{array}{c}\text { Right } \\
\text { Change }\end{array}$ & & $\begin{array}{c}\text { Left } \\
\text { Change }\end{array}$ & $\begin{array}{c}\text { Right } \\
\text { Change }\end{array}$ \\
\hline Edgewise, Female & 5.072 & 3.189 & Edgewise & 6.817 & 3.164 \\
\hline Edgewise, Male & 8.562 & 3.138 & MARA+Edgewise & 3.464 & 5.039 \\
\hline MARA+Edgewise, Female & 0.343 & 2.779 & & & \\
\hline MARA+Edgewise, Male & 6.584 & 7.300 & & & \\
\hline
\end{tabular}

Table C-16. Descriptive statistics (least-square means) of the sample partitioned by treatment and sex for the variable Sagittal MJS L Initial and Sagittal AJS R Initial.

\begin{tabular}{|c|c|c|c|c|c|}
\hline \multirow[b]{3}{*}{ Group } & \multicolumn{2}{|c|}{ Treatment-by-Sex } & & \multicolumn{2}{|c|}{ Sexes Combined } \\
\hline & Left & Right & & Left & Right \\
\hline & Initial & Initial & & Initial & Initial \\
\hline Edgewise, Female & 1.956 & 1.356 & Edgewise & 2.009 & 1.351 \\
\hline Edgewise, Male & 2.062 & 1.346 & MARA+Edgewise & 2.614 & 1.450 \\
\hline MARA+Edgewise, Female & 2.507 & 1.379 & & & \\
\hline MARA+Edgewise, Male & 2.721 & 1.521 & & & \\
\hline
\end{tabular}


Table C-17. Descriptive statistics (least-square means) of the sample partitioned by treatment and sex for the variable Sagittal MJS L Final and Sagittal MJS R Final.

\begin{tabular}{lccccc}
\hline & \multicolumn{2}{c}{ Treatment-by-Sex } & & \multicolumn{2}{c}{ Sexes Combined } \\
\multicolumn{1}{c}{ Left } & Reft & Right & Right \\
Final & Final & Final & & Final & Final \\
\hline Edgewise, Female & 2.111 & 2.417 & Edgewise & 2.259 & 2.497 \\
Edgewise, Male & 2.408 & 2.577 & MARA+Edgewise & 2.456 & 2.671 \\
MARA+Edgewise, Female & 2.143 & 2.479 & & \\
MARA+Edgewise, Male & 2.768 & 2.863 & & \\
\hline
\end{tabular}

Table C-18. Descriptive statistics (least-square means) of the sample partitioned by treatment and sex for the variable Sagittal AJS L Initial and Sagittal AJS R Initial.

\begin{tabular}{|c|c|c|c|c|c|}
\hline \multirow[b]{2}{*}{ Group } & \multicolumn{2}{|c|}{ Treatment-by-Sex } & & \multicolumn{2}{|c|}{ Sexes Combined } \\
\hline & $\begin{array}{c}\text { Left } \\
\text { Initial }\end{array}$ & $\begin{array}{l}\text { Right } \\
\text { Initial }\end{array}$ & & $\begin{array}{c}\text { Left } \\
\text { Initial }\end{array}$ & $\begin{array}{l}\text { Right } \\
\text { Initial }\end{array}$ \\
\hline Edgewise-Alone,Female & 1.256 & 1.356 & Edgewise-Alone & 1.262 & 1.351 \\
\hline Edgewise-Alone,Male & 1.269 & 1.346 & MARA+Edgewise & 1.424 & 1.450 \\
\hline MARA+Edgewise,Female & 1.421 & 1.379 & & & \\
\hline MARA+Edgewise,Male & 1.426 & 1.521 & & & \\
\hline
\end{tabular}


Table C-19. Descriptive statistics (least-square means) of the sample partitioned by treatment and sex for the variable Sagittal AJS L Final and Sagittal AJS R Final.

\begin{tabular}{|c|c|c|c|c|c|}
\hline \multirow[b]{2}{*}{ Group } & \multicolumn{2}{|c|}{ Treatment-by-Sex } & & \multicolumn{2}{|c|}{ Sexes Combined } \\
\hline & $\begin{array}{c}\text { Left } \\
\text { Final }\end{array}$ & $\begin{array}{l}\text { Right } \\
\text { Final }\end{array}$ & & $\begin{array}{l}\text { Left } \\
\text { Final }\end{array}$ & $\begin{array}{l}\text { Right } \\
\text { Final }\end{array}$ \\
\hline Edgewise, Female & 2.583 & 1.417 & Edgewise & 2.742 & 1.343 \\
\hline Edgewise, Male & 2.900 & 1.269 & MARA+Edgewise & 2.674 & 1.490 \\
\hline MARA+Edgewise, Female & 2.686 & 1.364 & & & \\
\hline MARA+Edgewise, Male & 2.663 & 1.616 & & & \\
\hline
\end{tabular}

Table C-20. Descriptive statistics (least-square means) of the sample partitioned by treatment and sex for the variable Change in Sagittal PJS L and Change in Sagittal PJS R.

\begin{tabular}{|c|c|c|c|c|c|}
\hline \multirow[b]{2}{*}{ Group } & \multicolumn{2}{|c|}{ Treatment-by-Sex } & & \multicolumn{2}{|c|}{ Sexes Combined } \\
\hline & $\begin{array}{c}\text { Left } \\
\text { Change }\end{array}$ & $\begin{array}{c}\text { Right } \\
\text { Change }\end{array}$ & & $\begin{array}{c}\text { Left } \\
\text { Change }\end{array}$ & $\begin{array}{l}\text { Right } \\
\text { Change }\end{array}$ \\
\hline Edgewise, Female & -0.161 & -0.061 & Edgewise & -0.034 & 0.119 \\
\hline Edgewise, Male & 0.092 & 0.300 & MARA+Edgewise & -0.199 & -0.349 \\
\hline MARA+Edgewise, Female & -0.336 & -0.550 & & & \\
\hline MARA+Edgewise, Male & -0.063 & -0.147 & & & \\
\hline
\end{tabular}


Table C-21. Descriptive statistics (least-square means) of the sample partitioned by treatment and sex for the variable Sagittal FW L Initial and Sagittal FW R Initial.

\begin{tabular}{|c|c|c|c|c|c|}
\hline \multirow[b]{3}{*}{ Group } & \multicolumn{2}{|c|}{ Treatment-by-Sex } & & \multicolumn{2}{|c|}{ Sexes Combined } \\
\hline & Left & Right & & Left & Right \\
\hline & Initial & Initial & & Initial & Initial \\
\hline Edgewise, Female & 17.383 & 17.917 & Edgewise & 17.496 & 18.097 \\
\hline Edgewise, Male & 17.608 & 18.277 & MARA+Edgewise & 17.208 & 16.751 \\
\hline MARA+Edgewise, Female & 17.257 & 16.864 & & & \\
\hline MARA+Edgewise, Male & 17.158 & 16.637 & & & \\
\hline
\end{tabular}

Table C-22. Descriptive statistics (least-square means) of the sample partitioned by treatment and sex for the variable Sagittal FW L Final and Sagittal FW R Final.

\begin{tabular}{|c|c|c|c|c|c|}
\hline \multirow[b]{3}{*}{ Group } & \multicolumn{2}{|c|}{ Treatment-by-Sex } & & \multicolumn{2}{|c|}{ Sexes Combined } \\
\hline & Left & Right & & Left & Right \\
\hline & Final & Final & & Final & Final \\
\hline Edgewise, Female & 17.478 & 18.100 & Edgewise & 17.635 & 18.500 \\
\hline Edgewise, Male & 17.792 & 18.900 & MARA+Edgewise & 17.498 & 16.945 \\
\hline MARA+Edgewise, Female & 17.121 & 16.486 & & & \\
\hline MARA+Edgewise, Male & 17.874 & 17.405 & & & \\
\hline
\end{tabular}


Table C-23. Descriptive statistics (least-square means) of the sample partitioned by treatment and sex for the variable Change in Sagittal FW L and Change in Sagittal FW R.

\begin{tabular}{|c|c|c|c|c|c|}
\hline \multirow[b]{2}{*}{ Group } & \multicolumn{2}{|c|}{ Treatment-by-Sex } & & \multicolumn{2}{|c|}{ Sexes Combined } \\
\hline & $\begin{array}{c}\text { Left } \\
\text { Change }\end{array}$ & $\begin{array}{c}\text { Right } \\
\text { Change }\end{array}$ & & $\begin{array}{c}\text { Left } \\
\text { Change }\end{array}$ & $\begin{array}{l}\text { Right } \\
\text { Change }\end{array}$ \\
\hline Edgewise, Female & 0.094 & 0.183 & Edgewise & 0.140 & 0.403 \\
\hline Edgewise, Male & 0.185 & 0.623 & MARA+Edgewise & 0.290 & 0.195 \\
\hline MARA+Edgewise, Female & -0.136 & -0.379 & & & \\
\hline MARA+Edgewise, Male & 0.716 & 0.768 & & & \\
\hline
\end{tabular}

Table C-24. Descriptive statistics (least-square means) of the sample partitioned by treatment and sex for the variable Sagittal FD L Initial and Sagittal FD R Initial.

\begin{tabular}{|c|c|c|c|c|c|}
\hline \multirow[b]{2}{*}{ Group } & \multicolumn{2}{|c|}{ Treatment-by-Sex } & & \multicolumn{2}{|c|}{ Sexes Combined } \\
\hline & $\begin{array}{c}\text { Left } \\
\text { Initial }\end{array}$ & $\begin{array}{l}\text { Right } \\
\text { Initial }\end{array}$ & & $\begin{array}{l}\text { Left } \\
\text { Initial }\end{array}$ & $\begin{array}{l}\text { Right } \\
\text { Initial }\end{array}$ \\
\hline Edgewise, Female & 7.017 & 7.372 & Edgewise & 7.070 & 7.371 \\
\hline Edgewise, Male & 7.123 & 7.369 & MARA+Edgewise & 7.839 & 7.830 \\
\hline MARA+Edgewise, Female & 7.636 & 7.729 & & & \\
\hline MARA+Edgewise, Male & 8.042 & 7.932 & & & \\
\hline
\end{tabular}


Table C-25. Descriptive statistics (least-square means) of the sample partitioned by treatment and sex for the variable Sagittal FD L Final and Sagittal FD R Final.

\begin{tabular}{lccccc}
\hline & \multicolumn{2}{c}{ Treatment-by-Sex } & & \multicolumn{2}{c}{ Sexes Combined } \\
\multicolumn{1}{c}{ Left } & Left & Right & & Right \\
Froup & Final & Final & & Final & Final \\
\hline Edgewise, Female & 7.506 & 7.761 & Edgewise & 7.499 & 7.700 \\
Edgewise, Male & 7.492 & 7.638 & MARA+Edgewise & 7.995 & 7.972 \\
MARA+Edgewise, Female & 7.843 & 7.886 & & \\
MARA+Edgewise, Male & 8.147 & 8.058 & & \\
\hline
\end{tabular}

Table C-26. Descriptive statistics (least-square means) of the sample partitioned by treatment and sex for the variable Change in Sagittal FD $L$ and Change in Sagittal FD R.

\begin{tabular}{lcccc}
\hline & \multicolumn{2}{c}{ Treatment-by-Sex } & \multicolumn{2}{c}{ Sexes Combined } \\
\cline { 2 - 5 } \multicolumn{1}{c}{ Group } & $\begin{array}{c}\text { Left } \\
\text { Change }\end{array}$ & $\begin{array}{c}\text { Right } \\
\text { Change }\end{array}$ & Right \\
Change & 0.429 & 0.329 \\
Change
\end{tabular}


Table C-27. Descriptive statistics (least-square means) of the sample partitioned by treatment and sex for the variable Sagittal FW L Initial and Sagittal FW R Initial.

\begin{tabular}{|c|c|c|c|c|c|}
\hline \multirow[b]{2}{*}{ Group } & \multicolumn{2}{|c|}{ Treatment-by-Sex } & & \multicolumn{2}{|c|}{ Sexes Combined } \\
\hline & $\begin{array}{l}\text { Left } \\
\text { Initial }\end{array}$ & $\begin{array}{l}\text { Right } \\
\text { Initial }\end{array}$ & & $\begin{array}{l}\text { Left } \\
\text { Initial }\end{array}$ & $\begin{array}{l}\text { Right } \\
\text { Initial }\end{array}$ \\
\hline Edgewise, Female & 17.383 & 17.917 & Edgewise & 17.496 & 18.097 \\
\hline Edgewise, Male & 17.608 & 18.277 & MARA+Edgewise & 17.208 & 16.751 \\
\hline MARA+Edgewise, Female & 17.257 & 16.864 & & & \\
\hline MARA+Edgewise, Male & 17.158 & 16.637 & & & \\
\hline
\end{tabular}

Table C-28. Descriptive statistics (least-square means) of the sample partitioned by treatment and sex for the variable Sagittal FW L Final and Sagittal FW R Final.

\begin{tabular}{|c|c|c|c|c|c|}
\hline & Trea & $y-\operatorname{Sex}$ & & Sexes & nbined \\
\hline & Left & Right & & Left & Right \\
\hline Group & Final & Final & & Final & Final \\
\hline Edgewise, Female & 17.478 & 18.100 & Edgewise & 17.635 & 18.500 \\
\hline Edgewise, Male & 17.792 & 18.900 & MARA+Edgewise & 17.498 & 16.945 \\
\hline MARA+Edgewise, Female & 17.121 & 16.486 & & & \\
\hline MARA+Edgewise, Male & 17.874 & 17.405 & & & \\
\hline
\end{tabular}


Table C-29. Descriptive statistics (least-square means) of the sample partitioned by treatment and sex for the variable Change in Sagittal FW L and Change in Sagittal FW R.

\begin{tabular}{|c|c|c|c|c|c|}
\hline \multirow[b]{2}{*}{ Group } & \multicolumn{2}{|c|}{ Treatment-by-Sex } & & \multicolumn{2}{|c|}{$\underline{\text { Sexes Combined }}$} \\
\hline & $\begin{array}{c}\text { Left } \\
\text { Change }\end{array}$ & $\begin{array}{l}\text { Right } \\
\text { Change }\end{array}$ & & $\begin{array}{c}\text { Left } \\
\text { Change }\end{array}$ & $\begin{array}{c}\text { Right } \\
\text { Change }\end{array}$ \\
\hline Edgewise, Female & 0.094 & 0.183 & Edgewise & 0.140 & 0.403 \\
\hline Edgewise, Male & 0.185 & 0.623 & MARA+Edgewise & 0.290 & 0.195 \\
\hline MARA+Edgewise, Female & -0.136 & -0.379 & & & \\
\hline MARA+Edgewise, Male & 0.716 & 0.768 & & & \\
\hline
\end{tabular}

Table C-30. Descriptive statistics (least-square means) of the sample partitioned by treatment and sex for the variable Sagittal CHH L Initial and Sagittal CHH R Initial.

\begin{tabular}{lccccc}
\hline \multicolumn{1}{c}{ Group } & \multicolumn{2}{c}{ Treatment-by-Sex } & \multicolumn{2}{c}{ Sexes Combined } \\
\cline { 3 - 5 } & $\begin{array}{c}\text { Left } \\
\text { Change }\end{array}$ & $\begin{array}{c}\text { Right } \\
\text { Change }\end{array}$ & Left & $\begin{array}{c}\text { Right } \\
\text { Change }\end{array}$ \\
Edgewise, Female & 6.400 & 6.494 & Edgewise & 6.596 & 6.732 \\
Edgewise, Male & 6.792 & 6.969 & MARA+Edgewise & 6.368 & 6.303 \\
MARA+Edgewise, Female & 5.979 & 6.100 & & \\
MARA+Edgewise, Male & 6.758 & 6.505 & & \\
\hline
\end{tabular}


Table C-31. Descriptive statistics (least-square means) of the sample partitioned by treatment and sex for the variable Sagittal CHH L Final and Sagittal CHH R Final.

\begin{tabular}{|c|c|c|c|c|c|}
\hline \multirow[b]{2}{*}{ Group } & \multicolumn{2}{|c|}{ Treatment-by-Sex } & & \multicolumn{2}{|c|}{ Sexes Combined } \\
\hline & $\begin{array}{c}\text { Left } \\
\text { Initial }\end{array}$ & $\begin{array}{l}\text { Right } \\
\text { Initial }\end{array}$ & & $\begin{array}{l}\text { Left } \\
\text { Initial }\end{array}$ & $\begin{array}{l}\text { Right } \\
\text { Initial }\end{array}$ \\
\hline Edgewise, Female & 6.361 & 6.983 & Edgewise & 6.661 & 7.176 \\
\hline Edgewise, Male & 6.962 & 7.369 & MARA+Edgewise & 6.538 & 6.489 \\
\hline MARA+Edgewise, Female & 6.207 & 6.293 & & & \\
\hline MARA+Edgewise, Male & 6.868 & 6.684 & & & \\
\hline
\end{tabular}

Table C-32. Descriptive statistics (least-square means) of the sample partitioned by treatment and sex for the variable Change in Sagittal CHH L and Change in Sagittal CHH R.

\begin{tabular}{|c|c|c|c|c|c|}
\hline \multirow[b]{2}{*}{ Group } & \multicolumn{2}{|c|}{ Treatment-by-Sex } & & \multicolumn{2}{|c|}{ Sexes Combined } \\
\hline & $\begin{array}{c}\text { Left } \\
\text { Change }\end{array}$ & $\begin{array}{l}\text { Right } \\
\text { Change }\end{array}$ & & $\begin{array}{c}\text { Left } \\
\text { Change }\end{array}$ & $\begin{array}{l}\text { Right } \\
\text { Change }\end{array}$ \\
\hline Edgewise, Female & -0.039 & 0.489 & Edgewise & 0.065 & 0.444 \\
\hline Edgewise, Male & 0.169 & 0.400 & MARA+Edgewise & 0.170 & 0.186 \\
\hline MARA+Edgewise, Female & 0.229 & 0.193 & & & \\
\hline MARA+Edgewise, Male & 0.111 & 0.179 & & & \\
\hline
\end{tabular}


Table C-33. Descriptive statistics (least-square means) of the sample partitioned by treatment and sex for the variable Sagittal EAM-AE L Initial and Sagittal EAM-AE R Initial.

\begin{tabular}{|c|c|c|c|c|c|}
\hline \multirow[b]{2}{*}{ Group } & \multicolumn{2}{|c|}{ Treatment-by-Sex } & & \multicolumn{2}{|c|}{ Sexes Combined } \\
\hline & $\begin{array}{l}\text { Left } \\
\text { Initial }\end{array}$ & $\begin{array}{l}\text { Right } \\
\text { Initial }\end{array}$ & & $\begin{array}{l}\text { Left } \\
\text { Initial }\end{array}$ & $\begin{array}{l}\text { Right } \\
\text { Initial }\end{array}$ \\
\hline Edgewise, Female & 22.761 & 24.072 & Edgewise & 23.123 & 24.582 \\
\hline Edgewise, Male & 23.485 & 25.092 & MARA+Edgewise & 22.864 & 22.627 \\
\hline MARA+Edgewise, Female & 22.964 & 22.664 & & & \\
\hline MARA+Edgewise, Male & 22.763 & 22.589 & & & \\
\hline
\end{tabular}

Table C-34. Descriptive statistics (least-square means) of the sample partitioned by treatment and sex for the variable Sagittal EAM-AE L Final and Sagittal EAM-AE R Final.

\begin{tabular}{lccccc}
\hline & \multicolumn{2}{c}{ Treatment-by-Sex } & & \multicolumn{2}{c}{ Sexes Combined } \\
\cline { 3 - 6 } \multicolumn{1}{c}{ Group } & Left & Right & & Left & Right \\
Final & Final \\
Edgewise, Female & Final & Final & & 23.995 & 25.005 \\
Edgewise, Male & 23.806 & 24.456 & Edgewise & 23.765 & 23.207 \\
MARA+Edgewise, Female & 24.185 & 25.554 & MARA+Edgewise & & \\
MARA+Edgewise, Male & 23.636 & 23.036 & & \\
\hline
\end{tabular}


Table C-35. Descriptive statistics (least-square means) of the sample partitioned by treatment and sex for the variable Change in Sagittal EAM AE $L$ and Change in Sagittal EAM AE R.

\begin{tabular}{|c|c|c|c|c|c|}
\hline \multirow[b]{2}{*}{ Group } & \multicolumn{2}{|c|}{ Treatment-by-Sex } & & \multicolumn{2}{|c|}{$\underline{\text { Sexes Combined }}$} \\
\hline & $\begin{array}{c}\text { Left } \\
\text { Change }\end{array}$ & $\begin{array}{c}\text { Right } \\
\text { Change }\end{array}$ & & $\begin{array}{c}\text { Left } \\
\text { Change }\end{array}$ & $\begin{array}{c}\text { Right } \\
\text { Change }\end{array}$ \\
\hline Edgewise, Female & 1.044 & 0.383 & Edgewise & 0.872 & 0.422 \\
\hline Edgewise, Male & 0.700 & 0.462 & MARA+Edgewise & 0.902 & 0.580 \\
\hline MARA+Edgewise, Female & 0.671 & 0.371 & & & \\
\hline MARA+Edgewise, Male & 1.132 & 0.789 & & & \\
\hline
\end{tabular}

Table C-36. Descriptive statistics (least-square means) of the sample partitioned by treatment and sex for the variable Sagittal EAM-C L Initial and Sagittal EAM-C R Initial.

\begin{tabular}{|c|c|c|c|c|c|}
\hline \multirow[b]{2}{*}{ Group } & \multicolumn{2}{|c|}{ Treatment-by-Sex } & & \multicolumn{2}{|c|}{ Sexes Combined } \\
\hline & $\begin{array}{c}\text { Left } \\
\text { Initial }\end{array}$ & $\begin{array}{l}\text { Right } \\
\text { Initial }\end{array}$ & & $\begin{array}{l}\text { Left } \\
\text { Initial }\end{array}$ & $\begin{array}{l}\text { Right } \\
\text { Initial }\end{array}$ \\
\hline Edgewise, Female & 12.489 & 13.528 & Edgewise & 12.791 & 13.756 \\
\hline Edgewise, Male & 13.092 & 13.985 & MARA+Edgewise & 13.255 & 13.009 \\
\hline MARA+Edgewise, Female & 13.479 & 13.186 & & & \\
\hline MARA+Edgewise, Male & 13.032 & 12.832 & & & \\
\hline
\end{tabular}


Table C-37. Descriptive statistics (least-square means) of the sample partitioned by treatment and sex for the variable Sagittal EAM-C L Final and Sagittal EAM-C R Final.

\begin{tabular}{lccccc}
\hline & \multicolumn{2}{c}{ Treatment-by-Sex } & & \multicolumn{2}{c}{ Sexes Combined } \\
\multicolumn{1}{c}{ Left } & Right & Right \\
Final & Final & Final & & Final \\
Edgewise, Female & 13.833 & 13.678 & Edgewise & 13.705 & 14.066 \\
Edgewise, Male & 13.577 & 14.454 & MARA+Edgewise & 13.428 & 13.127 \\
MARA+Edgewise, Female & 13.714 & 13.229 & & \\
MARA+Edgewise, Male & 13.142 & 13.026 & & \\
\hline
\end{tabular}

Table C-38. Descriptive statistics (least-square means) of the sample partitioned by treatment and sex for the variable Change in Sagittal EAM C L and Change in Sagittal EAM C R.

\begin{tabular}{|c|c|c|c|c|c|}
\hline \multirow[b]{2}{*}{ Group } & \multicolumn{2}{|c|}{ Treatment-by-Sex } & & \multicolumn{2}{|c|}{ Sexes Combined } \\
\hline & $\begin{array}{c}\text { Left } \\
\text { Change }\end{array}$ & $\begin{array}{l}\text { Right } \\
\text { Change }\end{array}$ & & $\begin{array}{c}\text { Left } \\
\text { Change }\end{array}$ & $\begin{array}{l}\text { Right } \\
\text { Change }\end{array}$ \\
\hline Edgewise, Female & 1.344 & 0.150 & Edgewise & 0.915 & 0.310 \\
\hline Edgewise, Male & 0.485 & 0.469 & MARA+Edgewise & 0.173 & 0.119 \\
\hline MARA+Edgewise, Female & 0.236 & 0.043 & & & \\
\hline MARA+Edgewise, Male & 0.111 & 0.195 & & & \\
\hline
\end{tabular}


Table C-39. Descriptive statistics (least-square means) of the sample partitioned by treatment and sex for the variable Sagittal EAM-P L Initial and Sagittal EAM-C R Initial.

\begin{tabular}{|c|c|c|c|c|c|}
\hline \multirow[b]{2}{*}{ Group } & \multicolumn{2}{|c|}{ Treatment-by-Sex } & & \multicolumn{2}{|c|}{ Sexes Combined } \\
\hline & $\begin{array}{l}\text { Left } \\
\text { Initial }\end{array}$ & $\begin{array}{l}\text { Right } \\
\text { Initial }\end{array}$ & & $\begin{array}{l}\text { Left } \\
\text { Initial }\end{array}$ & $\begin{array}{l}\text { Right } \\
\text { Initial }\end{array}$ \\
\hline Edgewise, Female & 5.489 & 13.528 & Edgewise & 5.441 & 13.756 \\
\hline Edgewise, Male & 5.392 & 13.985 & MARA+Edgewise & 5.839 & 13.009 \\
\hline MARA+Edgewise, Female & 5.957 & 13.186 & & & \\
\hline MARA+Edgewise, Male & 5.721 & 12.832 & & & \\
\hline
\end{tabular}

Table C-40. Descriptive statistics (least-square means) of the sample partitioned by treatment and sex for the variable Sagittal EAM-P L Final and Sagittal EAM-P R Final.

\begin{tabular}{lccccc}
\hline & \multicolumn{2}{c}{ Treatment-by-Sex } & & \multicolumn{2}{c}{ Sexes Combined } \\
\cline { 3 - 6 } \multicolumn{1}{c}{ Group } & Left & Right & & Left & Right \\
Final & Final \\
Edgewise, Female & Final & Final & & 5.919 & 6.137 \\
Edgewise, Male & 6.022 & 5.967 & Edgewise & 6.199 & 6.047 \\
MARA+Edgewise, Female & 5.815 & 6.308 & MARA+Edgewise & & \\
MARA+Edgewise, Male & 6.450 & 6.079 & & \\
\hline
\end{tabular}


Table C-41. Descriptive statistics (least-square means) of the sample partitioned by treatment and sex for the variable Change in Sagittal EAM P L and Change in EAM P R.

\begin{tabular}{|c|c|c|c|c|c|}
\hline \multirow[b]{2}{*}{ Group } & \multicolumn{2}{|c|}{ Treatment-by-Sex } & & \multicolumn{2}{|c|}{$\underline{\text { Sexes Combined }}$} \\
\hline & $\begin{array}{c}\text { Left } \\
\text { Change }\end{array}$ & $\begin{array}{c}\text { Right } \\
\text { Change }\end{array}$ & & $\begin{array}{c}\text { Left } \\
\text { Change }\end{array}$ & $\begin{array}{c}\text { Right } \\
\text { Change }\end{array}$ \\
\hline Edgewise, Female & 0.533 & 0.067 & Edgewise & 0.478 & -0.001 \\
\hline Edgewise, Male & 0.423 & -0.069 & MARA+Edgewise & 0.360 & 0.184 \\
\hline MARA+Edgewise, Female & 0.493 & 0.136 & & & \\
\hline MARA+Edgewise, Male & 0.226 & 0.232 & & & \\
\hline
\end{tabular}

Table C-42. Descriptive statistics (least-square means) of the sample partitioned by treatment and sex for the variable Sagittal ATA L Initial and Sagittal ATA R Initial.

\begin{tabular}{|c|c|c|c|c|c|}
\hline \multirow[b]{2}{*}{ Group } & \multicolumn{2}{|c|}{ Treatment-by-Sex } & & \multicolumn{2}{|c|}{ Sexes Combined } \\
\hline & $\begin{array}{l}\text { Left } \\
\text { Initial }\end{array}$ & $\begin{array}{l}\text { Right } \\
\text { Initial }\end{array}$ & & $\begin{array}{c}\text { Left } \\
\text { Initial }\end{array}$ & $\begin{array}{l}\text { Right } \\
\text { Initial }\end{array}$ \\
\hline Edgewise, Female & 36.544 & 37.256 & Edgewise & 36.803 & 36.605 \\
\hline Edgewise, Male & 37.062 & 35.954 & MARA+Edgewise & 43.948 & 44.862 \\
\hline MARA+Edgewise, Female & 43.929 & 45.314 & & & \\
\hline MARA+Edgewise, Male & 43.968 & 44.411 & & & \\
\hline
\end{tabular}


Table C-43. Descriptive statistics (least-square means) of the sample partitioned by treatment and sex for the variable Sagittal ATA L Final and Sagittal ATA R Final.

\begin{tabular}{lccccc}
\hline & \multicolumn{2}{c}{ Treatment-by-Sex } & & \multicolumn{2}{c}{ Sexes Combined } \\
\multicolumn{1}{c}{ Left } & Right & & Right \\
Fin & Final & Final & & Final & Final \\
\hline Edgewise, Female & 38.239 & 39.394 & Edgewise & 37.854 & 38.020 \\
Edgewise, Male & 37.469 & 36.646 & MARA+Edgewise & 42.010 & 42.933 \\
MARA+Edgewise, Female & 43.179 & 43.893 & & \\
MARA+Edgewise, Male & 40.842 & 41.974 & & \\
\hline
\end{tabular}

Table C-44. Descriptive statistics (least-square means) of the sample partitioned by treatment and sex for the variable Change in Sagittal ATA $L$ and Change in Sagittal ATA R.

\begin{tabular}{|c|c|c|c|c|c|}
\hline \multirow[b]{2}{*}{ Group } & \multicolumn{2}{|c|}{ Treatment-by-Sex } & & \multicolumn{2}{|c|}{ Sexes Combined } \\
\hline & $\begin{array}{c}\text { Left } \\
\text { Change }\end{array}$ & $\begin{array}{c}\text { Right } \\
\text { Change }\end{array}$ & & $\begin{array}{c}\text { Left } \\
\text { Change }\end{array}$ & $\begin{array}{l}\text { Right } \\
\text { Change }\end{array}$ \\
\hline Edgewise, Female & 1.694 & 2.139 & Edgewise & 1.051 & 1.416 \\
\hline Edgewise, Male & 0.408 & 0.692 & MARA+Edgewise & -1.938 & -1.929 \\
\hline MARA+Edgewise, Female & -0.750 & -1.421 & & & \\
\hline MARA+Edgewise, Male & -3.126 & -2.437 & & & \\
\hline
\end{tabular}


Table C-45. Descriptive statistics (least-square means) of the sample partitioned by treatment and sex for the variable Sagittal CHA L Initial and Sagittal CHA R Initial.

\begin{tabular}{|c|c|c|c|c|c|}
\hline \multirow[b]{3}{*}{ Group } & \multicolumn{2}{|c|}{ Treatment-by-Sex } & & \multicolumn{2}{|c|}{$\underline{\text { Sexes Combined }}$} \\
\hline & $\overline{\text { Left }}$ & Right & & Left & Right \\
\hline & Initial & Initial & & Initial & Initial \\
\hline Edgewise-Alone,Female & 140.222 & 143.644 & Edgewise-Alone & 141.853 & 144.061 \\
\hline Edgewise-Alone,Male & 143.485 & 144.477 & MARA+Edgewise & 141.404 & 137.986 \\
\hline MARA+Edgewise,Female & 142.850 & 138.43 & & & \\
\hline MARA+Edgewise,Male & 139.958 & 137.537 & & & \\
\hline
\end{tabular}

Table C-46. Descriptive statistics (least-square means) of the sample partitioned by treatment and sex for the variable Sagittal CHA L Final and Sagittal CHA R Final.

\begin{tabular}{lccccc}
\hline & \multicolumn{2}{c}{ Treatment-by-Sex } & & \multicolumn{2}{c}{ Sexes Combined } \\
\cline { 3 - 6 } \multicolumn{1}{c}{ Group } & Left & Right & & Left \\
Finht & Final \\
Final & Final & & Edgewise-Alone & 135.701 & 139.010 \\
Edgewise-Alone,Female & 132.056 & 137.050 & MARA+Edgewise & 133.828 & 132.227 \\
MARA+Edgewise,Female & 139.346 & 140.969 & & \\
MARA+Edgewise,Male & 136.171 & 134.864 & &
\end{tabular}


Table C-47. Descriptive statistics (least-square means) of the sample partitioned by treatment and sex for the variable change in Sagittal CHA $L$ and change in Sagittal CHA R.

\begin{tabular}{lccccc}
\hline & \multicolumn{2}{c}{$\begin{array}{c}\text { Treatment-by-Sex } \\
\text { Left } \\
\text { Group }\end{array}$} & $\begin{array}{c}\text { Right } \\
\text { Initial }\end{array}$ & Sexes Combined \\
Initial & & $\begin{array}{c}\text { Left } \\
\text { Initial }\end{array}$ & $\begin{array}{c}\text { Right } \\
\text { Initial }\end{array}$ \\
Edgewise-Alone,Female & -8.167 & -6.594 & Edgewise-Alone & -6.153 & -5.051 \\
Edgewise-Alone,Male & -4.138 & -3.508 & MARA+Edgewise & -7.576 & -5.759 \\
MARA+Edgewise,Female & -6.679 & -3.571 & & \\
MARA+Edgewise,Male & -8.474 & -7.947 & & \\
\hline
\end{tabular}

Table C-48. Descriptive statistics (least-square means) of the sample partitioned by treatment and sex for the variable Sagittal ACP L Initial and Sagittal ACP R Initial.

\begin{tabular}{|c|c|c|c|c|c|}
\hline \multirow[b]{2}{*}{ Group } & \multicolumn{2}{|c|}{ Treatment-by-Sex } & & \multicolumn{2}{|c|}{ Sexes Combined } \\
\hline & $\begin{array}{c}\text { Left } \\
\text { Initial }\end{array}$ & $\begin{array}{l}\text { Right } \\
\text { Initial }\end{array}$ & & $\begin{array}{l}\text { Left } \\
\text { Initial }\end{array}$ & $\begin{array}{l}\text { Right } \\
\text { Initial }\end{array}$ \\
\hline Edgewise, Female & 48.600 & 49.850 & Edgewise & 48.665 & 49.179 \\
\hline Edgewise, Male & 48.731 & 48.508 & MARA+Edgewise & 42.239 & 41.660 \\
\hline MARA+Edgewise, Female & 39.257 & 39.293 & & & \\
\hline MARA+Edgewise, Male & 45.221 & 44.026 & & & \\
\hline
\end{tabular}


Table C-49. Descriptive statistics (least-square means) of the sample partitioned by treatment and sex for the variable Sagittal ACP L Final and Sagittal ACP R Final.

\begin{tabular}{lccccc}
\hline & \multicolumn{2}{c}{ Treatment-by-Sex } & & \multicolumn{2}{c}{ Sexes Combined } \\
\multicolumn{1}{c}{ Left } & Right & & Right \\
Final & Final & Final & & Final & Final \\
\hline Edgewise, Female & 48.628 & 47.450 & Edgewise & 50.499 & 50.560 \\
Edgewise, Male & 52.369 & 53.669 & MARA+Edgewise & 41.512 & 41.884 \\
MARA+Edgewise, Female & 37.593 & 39.500 & & \\
MARA+Edgewise, Male & 45.432 & 44.268 & & \\
\hline
\end{tabular}

Table C-50. Descriptive statistics (least-square means) of the sample partitioned by treatment and sex for the variable Change in ACP $L$ and Change in Sagittal ACP R.

\begin{tabular}{|c|c|c|c|c|c|}
\hline \multirow[b]{2}{*}{ Group } & \multicolumn{2}{|c|}{ Treatment-by-Sex } & & \multicolumn{2}{|c|}{ Sexes Combined } \\
\hline & $\begin{array}{c}\text { Left } \\
\text { Change }\end{array}$ & $\begin{array}{c}\text { Right } \\
\text { Change }\end{array}$ & & $\begin{array}{c}\text { Left } \\
\text { Change }\end{array}$ & $\begin{array}{l}\text { Right } \\
\text { Change }\end{array}$ \\
\hline Edgewise, Female & 0.028 & -2.400 & Edgewise & 1.833 & 1.381 \\
\hline Edgewise, Male & 3.638 & 5.162 & MARA+Edgewise & -0.727 & 0.225 \\
\hline MARA+Edgewise, Female & -1.664 & 0.207 & & & \\
\hline MARA+Edgewise, Male & 0.211 & 0.242 & & & \\
\hline
\end{tabular}


Table C-51. Descriptive statistics (least-square means) of the sample partitioned by treatment and sex for the variable Sagittal AAE L Initial and Sagittal AAE R Initial.

\begin{tabular}{|c|c|c|c|c|c|}
\hline \multirow[b]{3}{*}{ Group } & \multicolumn{2}{|c|}{$\underline{\text { Treatment-by-Sex }}$} & & \multicolumn{2}{|c|}{ Sexes Combined } \\
\hline & Left & Right & & Left & Right \\
\hline & Initial & Initial & & Initial & Initial \\
\hline Edgewise, Female & 82.811 & 79.983 & Edgewise & 86.736 & 87.738 \\
\hline Edgewise, Male & 90.662 & 95.492 & MARA+Edgewise & 89.428 & 96.289 \\
\hline MARA+Edgewise, Female & 86.414 & 95.336 & & & \\
\hline MARA+Edgewise, Male & 92.442 & 97.242 & & & \\
\hline
\end{tabular}

Table C-52. Descriptive statistics (least-square means) of the sample partitioned by treatment and sex for the variable Sagittal AAE L Final and Sagittal AAE R Final.

\begin{tabular}{|c|c|c|c|c|c|}
\hline \multirow[b]{3}{*}{ Group } & \multicolumn{2}{|c|}{ Treatment-by-Sex } & & \multicolumn{2}{|c|}{ Sexes Combined } \\
\hline & Left & Right & & Left & Right \\
\hline & Final & Final & & Final & Final \\
\hline Edgewise-Alone,Female & 95.061 & 92.339 & Edgewise-Alone & 97.604 & 98.281 \\
\hline Edgewise-Alone,Male & 100.146 & 104.223 & MARA+Edgewise & 99.048 & 102.240 \\
\hline MARA+Edgewise,Female & 94.543 & 102.464 & & & \\
\hline MARA+Edgewise,Male & 103.553 & 102.016 & & & \\
\hline
\end{tabular}


Table C-53. Descriptive statistics (least-square means) of the sample partitioned by treatment and sex for the variable Change in Sagittal AAE $L$ and Change in Sagittal AAE R.

\begin{tabular}{lrrrrr} 
& \multicolumn{2}{c}{ Treatment-by-Sex } & & \multicolumn{2}{c}{ Sexes Combined } \\
\multicolumn{1}{c}{ Left } & Right & & $\begin{array}{c}\text { Right } \\
\text { Left }\end{array}$ \\
& Change & Change & & Change & Change \\
Edgewise, Female & 12.250 & 12.356 & Edgewise-Alone & 10.867 & 10.543 \\
Edgewise, Male & 9.485 & 8.731 & MARA+Edgewise & 9.620 & 5.951 \\
MARA+Edgewise, Female & 8.129 & 7.129 & & \\
MARA+Edgewise, Male & 11.111 & 4.774 & & \\
\hline
\end{tabular}

Table C-54. Descriptive statistics (least-square means) of the sample partitioned by treatment and sex for the variable Sagittal AGF L Initial and Sagittal AGF R Initial.

\begin{tabular}{|c|c|c|c|c|c|}
\hline \multirow[b]{2}{*}{ Group } & \multicolumn{2}{|c|}{ Treatment-by-Sex } & & \multicolumn{2}{|c|}{ Sexes Combined } \\
\hline & $\begin{array}{c}\text { Left } \\
\text { Initial }\end{array}$ & $\begin{array}{l}\text { Right } \\
\text { Initial }\end{array}$ & & $\begin{array}{l}\text { Left } \\
\text { Initial }\end{array}$ & $\begin{array}{l}\text { Right } \\
\text { Initial }\end{array}$ \\
\hline Edgewise, Female & 70.522 & 78.322 & Edgewise-Alone & 69.834 & 78.253 \\
\hline Edgewise, Male & 69.146 & 78.185 & MARA+Edgewise & 82.147 & 82.561 \\
\hline MARA+Edgewise, Female & 80.336 & 83.086 & & & \\
\hline MARA+Edgewise, Male & 83.958 & 82.037 & & & \\
\hline
\end{tabular}


Table C-55. Descriptive statistics (least-square means) of the sample partitioned by treatment and sex for the variable Sagittal AGF L Final and Sagittal AGF R Final.

\begin{tabular}{lccccc}
\hline & \multicolumn{2}{c}{ Treatment-by-Sex } & & \multicolumn{2}{c}{ Sexes Combined } \\
\multicolumn{1}{c}{ Left } & Left & Right & & Right \\
Final & Final & & Final & Final \\
\hline Edgewise, Female & 78.828 & 86.383 & Edgewise & 77.687 & 84.707 \\
Edgewise, Male & 76.546 & 83.031 & MARA+Edgewise & 81.466 & 84.021 \\
MARA+Edgewise, Female & 78.843 & 83.921 & & \\
MARA+Edgewise, Male & 84.089 & 84.121 & & \\
\hline
\end{tabular}

Table C-56. Descriptive statistics (least-square means) of the sample partitioned by treatment and sex for the variable Change in Sagittal AGF $L$ and Change in Sagittal AGF R.

\begin{tabular}{|c|c|c|c|c|c|}
\hline \multirow[b]{2}{*}{ Group } & \multicolumn{2}{|c|}{ Treatment-by-Sex } & & \multicolumn{2}{|c|}{ Sexes Combined } \\
\hline & $\begin{array}{c}\text { Left } \\
\text { Change }\end{array}$ & $\begin{array}{l}\text { Right } \\
\text { Change }\end{array}$ & & $\begin{array}{c}\text { Left } \\
\text { Change }\end{array}$ & $\begin{array}{l}\text { Right } \\
\text { Change }\end{array}$ \\
\hline Edgewise, Female & 8.306 & 8.061 & Edgewise & 7.853 & 6.454 \\
\hline Edgewise, Male & 7.400 & 4.846 & MARA+Edgewise & -0.681 & 1.460 \\
\hline MARA+Edgewise, Female & -1.493 & 0.836 & & & \\
\hline MARA+Edgewise, Male & 0.132 & 2.084 & & & \\
\hline
\end{tabular}


Table C-57. Descriptive statistics (least-square means) of the sample partitioned by treatment and sex for the variable Coronal MJS L Initial and Coronal MJS R Initial.

\begin{tabular}{|c|c|c|c|c|c|}
\hline \multirow[b]{3}{*}{ Group } & \multicolumn{2}{|c|}{ Treatment-by-Sex } & & \multicolumn{2}{|c|}{$\underline{\text { Sexes Combined }}$} \\
\hline & Left & Right & & Left & Right \\
\hline & Initial & Initial & & Initial & Initial \\
\hline Edgewise, Female & 1.933 & 1.800 & Edgewise & 1.844 & 1.758 \\
\hline Edgewise, Male & 1.754 & 1.715 & MARA+Edgewise & 1.880 & 1.821 \\
\hline MARA+Edgewise, Female & 1.929 & 1.821 & & & \\
\hline MARA+Edgewise, Male & 1.832 & 1.821 & & & \\
\hline
\end{tabular}

Table C-58. Descriptive statistics (least-square means) of the sample partitioned by treatment and sex for the variable Coronal MJS L Final and Coronal MJS R Final.

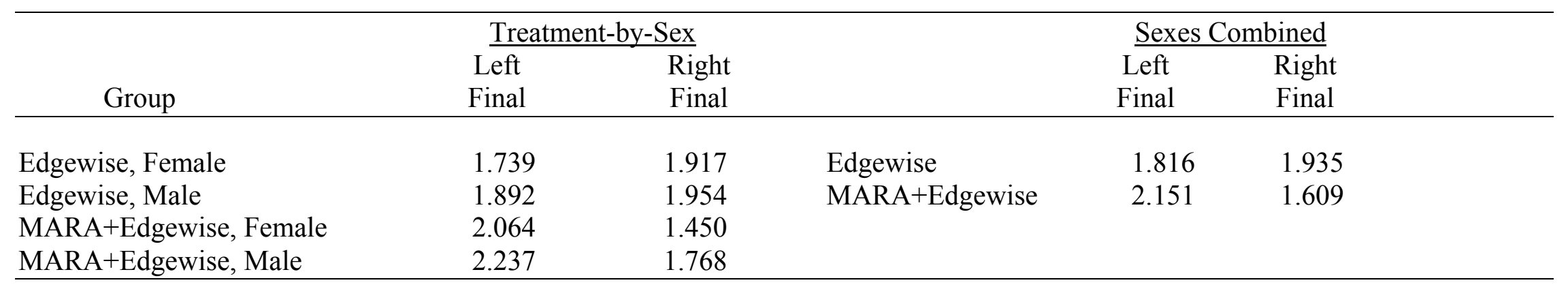


Table C-59. Descriptive statistics (least-square means) of the sample partitioned by treatment and sex for the variable Change in Coronal MJS L and Change in Coronal MJS R.

\begin{tabular}{|c|c|c|c|c|c|}
\hline \multirow[b]{2}{*}{ Group } & \multicolumn{2}{|c|}{ Treatment-by-Sex } & & \multicolumn{2}{|c|}{$\underline{\text { Sexes Combined }}$} \\
\hline & $\begin{array}{c}\text { Left } \\
\text { Change }\end{array}$ & $\begin{array}{c}\text { Right } \\
\text { Change }\end{array}$ & & $\begin{array}{c}\text { Left } \\
\text { Change }\end{array}$ & $\begin{array}{c}\text { Right } \\
\text { Change }\end{array}$ \\
\hline Edgewise, Female & -0.194 & 0.117 & Edgewise & -0.028 & 0.178 \\
\hline Edgewise, Male & 0.138 & 0.238 & MARA+Edgewise & 0.270 & -0.212 \\
\hline MARA+Edgewise, Female & 0.136 & -0.371 & & & \\
\hline MARA+Edgewise, Male & 0.405 & -0.053 & & & \\
\hline
\end{tabular}

Table C-60. Descriptive statistics (least-square means) of the sample partitioned by treatment and sex for the variable Coronal SJS L Initial and Coronal SJS R Initial.

\begin{tabular}{|c|c|c|c|c|c|}
\hline \multirow[b]{2}{*}{ Group } & \multicolumn{2}{|c|}{$\underline{\text { Treatment-by-Sex }}$} & & \multicolumn{2}{|c|}{$\underline{\text { Sexes Combined }}$} \\
\hline & $\begin{array}{c}\text { Left } \\
\text { Initial }\end{array}$ & $\begin{array}{l}\text { Right } \\
\text { Initial }\end{array}$ & & $\begin{array}{c}\text { Left } \\
\text { Initial }\end{array}$ & $\begin{array}{l}\text { Right } \\
\text { Initial }\end{array}$ \\
\hline Edgewise, Female & 1.939 & 1.833 & Edgewise & 1.985 & 1.859 \\
\hline Edgewise, Male & 2.031 & 1.885 & MARA+Edgewise & 1.780 & 1.945 \\
\hline MARA+Edgewise, Female & 1.807 & 1.943 & & & \\
\hline MARA+Edgewise, Male & 1.753 & 1.947 & & & \\
\hline
\end{tabular}


Table C-61. Descriptive statistics (least-square means) of the sample partitioned by treatment and sex for the variable Coronal SJS L Final and Coronal SJS R Final.

\begin{tabular}{lccccc}
\hline & \multicolumn{2}{c}{ Treatment-by-Sex } & & \multicolumn{2}{c}{ Sexes Combined } \\
\multicolumn{1}{c}{ Left } & Right & & Right \\
Final & Final & & Final & Final \\
\hline Edgewise, Female & 2.006 & 1.900 & Edgewise & 1.968 & 1.927 \\
Edgewise, Male & 1.931 & 1.954 & MARA+Edgewise & 1.797 & 1.673 \\
MARA+Edgewise, Female & 1.557 & 1.593 & & \\
MARA+Edgewise, Male & 2.037 & 1.753 & & \\
\hline
\end{tabular}

Table C-62. Descriptive statistics (least-square means) of the sample partitioned by treatment and sex for the variable Change in Coronal SJS L and Change in Coronal SJS R.

\begin{tabular}{lccccc}
\hline & \multicolumn{2}{c}{$\frac{\text { Treatment-by-Sex }}{\text { Left }}$} & Right & & \multicolumn{2}{c}{ Sexes Combined } \\
Left & Right \\
Change & Change & Change & Change \\
\hline Edgewise, Female & 0.067 & 0.067 & Edgewise & -0.017 & 0.068 \\
Edgewise, Male & -0.100 & 0.069 & MARA+Edgewise & 0.017 & -0.272 \\
MARA+Edgewise, Female & -0.250 & -0.350 & & \\
MARA+Edgewise, Male & 0.284 & -0.195 & & \\
\hline
\end{tabular}


Table C-63. Descriptive statistics (least-square means) of the sample partitioned by treatment and sex for the variable Coronal LJS L Initial and Coronal LJS R Initial.

\begin{tabular}{|c|c|c|c|c|c|}
\hline \multirow[b]{2}{*}{ Group } & \multicolumn{2}{|c|}{ Treatment-by-Sex } & & \multicolumn{2}{|c|}{ Sexes Combined } \\
\hline & $\begin{array}{c}\text { Left } \\
\text { Initial }\end{array}$ & $\begin{array}{l}\text { Right } \\
\text { Initial }\end{array}$ & & $\begin{array}{l}\text { Left } \\
\text { Initial }\end{array}$ & $\begin{array}{l}\text { Right } \\
\text { Initial }\end{array}$ \\
\hline Edgewise, Female & 2.689 & 2.489 & Edgewise & 2.648 & 2.468 \\
\hline Edgewise, Male & 2.608 & 2.446 & MARA+Edgewise & 2.294 & 2.275 \\
\hline MARA+Edgewise, Female & 2.271 & 2.350 & & & \\
\hline MARA+Edgewise, Male & 2.316 & 2.200 & & & \\
\hline
\end{tabular}

Table C-64. Descriptive statistics (least-square means) of the sample partitioned by treatment and sex for the variable Coronal LJS L Final and Coronal LJS R Final.

\begin{tabular}{lccccc}
\hline & \multicolumn{2}{c}{ Treatment-by-Sex } & & \multicolumn{2}{c}{ Sexes Combined } \\
\cline { 3 - 5 } \multicolumn{1}{c}{ Group } & Left & Right & & $\begin{array}{c}\text { Right } \\
\text { Lint } \\
\text { Final }\end{array}$ \\
\hline Edgewise, Female & Final & Final & & & 2.538 \\
Edgewise, Male & 2.483 & 2.439 & Edgewise & 2.569 \\
MARA+Edgewise, Female & 2.592 & 2.700 & MARA+Edgewise & 2.337 & 2.323 \\
MARA+Edgewise, Male & 2.143 & 2.371 & & \\
\hline
\end{tabular}


Table C-65. Descriptive statistics (least-square means) of the sample partitioned by treatment and sex for the variable Change in Coronal LJS $\mathrm{L}$ and Change in Coronal LJS R.

\begin{tabular}{|c|c|c|c|c|c|}
\hline \multirow[b]{2}{*}{ Group } & \multicolumn{2}{|c|}{ Treatment-by-Sex } & & \multicolumn{2}{|c|}{ Sexes Combined } \\
\hline & $\begin{array}{c}\text { Left } \\
\text { Change }\end{array}$ & $\begin{array}{l}\text { Right } \\
\text { Change }\end{array}$ & & $\begin{array}{c}\text { Left } \\
\text { Change }\end{array}$ & $\begin{array}{l}\text { Right } \\
\text { Change }\end{array}$ \\
\hline Edgewise, Female & -0.206 & -0.050 & Edgewise & -0.110 & 0.102 \\
\hline Edgewise, Male & -0.015 & 0.254 & MARA+Edgewise & 0.044 & 0.048 \\
\hline MARA+Edgewise, Female & -0.129 & 0.021 & & & \\
\hline MARA+Edgewise, Male & 0.216 & 0.074 & & & \\
\hline
\end{tabular}




\section{APPENDIX D. RESULTS OF PAIRED T-TESTS ASSESSING FOR SYSTEMATIC MEASUREMENT DIFFERENCES IN THE INTRAOBSERVER REPETITIONS}

Table D-1. Assessment of intraobserver repeatability for the variable Overjet Final.

\begin{tabular}{lc}
\hline \multicolumn{1}{c}{ Statistic } & Value \\
\hline Session Two (mean) & 2.6750 \\
Session One (mean) & 2.5750 \\
Mean Difference & 0.1000 \\
Standard Error & 0.1066 \\
Upper 95\% & 0.3346 \\
Lower 95\% & -0.1346 \\
Sample size & 12 \\
Correlation & 0.9401 \\
Paired t test & 0.9381 \\
Degrees freedom & 11 \\
P value (2 tail) & 0.3683 \\
\hline
\end{tabular}

Table D-2. Assessment of intraobserver repeatability for the variable ANB Final.

\begin{tabular}{lc}
\hline \multicolumn{1}{c}{ Statistic } & Value \\
\hline Session Two (mean) & 4.8500 \\
Session One (mean) & 4.7333 \\
Mean Difference & 0.1167 \\
Standard Error & 0.0767 \\
Upper 95\% & 0.2855 \\
Lower 95\% & -0.0522 \\
Sample size & 12 \\
Correlation & 0.9900 \\
Paired t test & 1.5210 \\
Degrees freedom & 11 \\
P value (2 tail) & 0.1565 \\
\hline
\end{tabular}


Table D-3. Assessment of intraobserver repeatability for the variable ANB Initial.

\begin{tabular}{lc}
\hline \multicolumn{1}{c}{ Statistic } & Value \\
\hline Session Two (mean) & \\
Session One (mean) & 5.5250 \\
Mean Difference & 5.7000 \\
Standard Error & -0.1750 \\
Upper 95\% & 0.0552 \\
Lower 95\% & -0.0535 \\
Sample size & -0.2965 \\
Correlation & 12 \\
Paired t test & 0.9926 \\
Degrees freedom & -3.1691 \\
P value (2 tail) & 11 \\
\hline
\end{tabular}

Table D-4. Assessment of intraobserver repeatability for the variable Axial AP L Final.

\begin{tabular}{lc}
\hline \multicolumn{1}{c}{ Statistic } & Value \\
\hline & \\
Session Two (mean) & 6.5083 \\
Session One (mean) & 6.4250 \\
Mean Difference & 0.0833 \\
Standard Error & 0.0705 \\
Upper 95\% & 0.2386 \\
Lower 95\% & -0.0719 \\
Sample size & 12 \\
Correlation & 0.9521 \\
Paired t test & 1.1815 \\
Degrees freedom & 11 \\
P value (2 tail) & 0.2623 \\
\hline
\end{tabular}


Table D-5. Assessment of intraobserver repeatability for the variable Axial AP L Initial.

\begin{tabular}{lc}
\hline \multicolumn{1}{c}{ Statistic } & Value \\
\hline Session Two (mean) & 6.5667 \\
Session One (mean) & 6.4083 \\
Mean Difference & 0.1583 \\
Standard Error & 0.1190 \\
Upper 95\% & 0.4202 \\
Lower 95\% & -0.1036 \\
Sample size & 12 \\
Correlation & 0.8863 \\
Paired t test & 1.3306 \\
Degrees freedom & 11 \\
P value (2 tail) & 0.2103 \\
\hline
\end{tabular}

Table D-6. Assessment of intraobserver repeatability for the variable Axial AP R Final.

\begin{tabular}{lc}
\hline \multicolumn{1}{c}{ Statistic } & Value \\
\hline & \\
Session Two (mean) & 6.2583 \\
Session One (mean) & 6.0417 \\
Mean Difference & 0.2167 \\
Standard Error & 0.1218 \\
Upper 95\% & 0.4846 \\
Lower 95\% & -0.0513 \\
Sample size & 12 \\
Correlation & 0.8756 \\
Paired t test & 1.7796 \\
Degrees freedom & 11 \\
P value (2 tail) & 0.1028 \\
\hline
\end{tabular}


Table D-7. Assessment of intraobserver repeatability for the variable Axial AP R Initial.

\begin{tabular}{lc}
\hline \multicolumn{1}{c}{ Statistic } & Value \\
\hline Session Two (mean) & 6.6000 \\
Session One (mean) & 6.4667 \\
Mean Difference & 0.1333 \\
Standard Error & 0.1257 \\
Upper 95\% & 0.4101 \\
Lower 95\% & -0.1434 \\
Sample size & 12 \\
Correlation & 0.7776 \\
Paired t test & 1.0605 \\
Degrees freedom & 11 \\
P value (2 tail) & 0.3117 \\
\hline
\end{tabular}

Table D-8. Assessment of intraobserver repeatability for the variable Axial APCD Final.

\begin{tabular}{lc}
\hline \multicolumn{1}{c}{ Statistic } & Value \\
\hline & \\
Session Two (mean) & -0.5333 \\
Session One (mean) & -0.5417 \\
Mean Difference & 0.0083 \\
Standard Error & 0.1777 \\
Upper 95\% & 0.3995 \\
Lower 95\% & -0.3828 \\
Sample size & 12 \\
Correlation & 0.9707 \\
Paired t test & 0.0469 \\
Degrees freedom & 11 \\
P value (2 tail) & 0.9634 \\
\hline
\end{tabular}


Table D-9. Assessment of intraobserver repeatability for the variable Axial APCD Initial.

\begin{tabular}{lc}
\hline \multicolumn{1}{c}{ Statistic } & Value \\
\hline Session Two (mean) & \\
Session One (mean) & 0.4417 \\
Mean Difference & 0.7333 \\
Standard Error & -0.2917 \\
Upper 95\% & 0.1848 \\
Lower 95\% & 0.1151 \\
Sample size & -0.6985 \\
Correlation & 12 \\
Paired t test & 0.9484 \\
Degrees freedom & -1.5781 \\
P value (2 tail) & 11 \\
\hline
\end{tabular}

Table D-10. Assessment of intraobserver repeatability for the variable Axial CA L Final.

\begin{tabular}{lc}
\hline \multicolumn{1}{c}{ Statistic } & Value \\
\hline & \\
Session Two (mean) & 61.2833 \\
Session One (mean) & 60.7833 \\
Mean Difference & 0.5000 \\
Standard Error & 0.8465 \\
Upper 95\% & 2.3631 \\
Lower 95\% & -1.3631 \\
Sample size & 12 \\
Correlation & 0.8427 \\
Paired t test & 0.5907 \\
Degrees freedom & 11 \\
P value (2 tail) & 0.5667 \\
\hline
\end{tabular}


Table D-11. Assessment of intraobserver repeatability for the variable Axial CA L Initial.

\begin{tabular}{lc}
\hline \multicolumn{1}{c}{ Statistic } & Value \\
\hline Session Two (mean) & 60.0500 \\
Session One (mean) & 59.6667 \\
Mean Difference & 0.3833 \\
Standard Error & 0.7393 \\
Upper 95\% & 2.0104 \\
Lower 95\% & -1.2437 \\
Sample size & 12 \\
Correlation & 0.8924 \\
Paired t test & 0.5185 \\
Degrees freedom & 11 \\
P value (2 tail) & 0.6143 \\
\hline
\end{tabular}

Table D-12. Assessment of intraobserver repeatability for the variable Axial CA R Final.

\begin{tabular}{lc}
\hline \multicolumn{1}{c}{ Statistic } & Value \\
\hline & \\
Session Two (mean) & 59.8500 \\
Session One (mean) & 60.4167 \\
Mean Difference & -0.5667 \\
Standard Error & 0.5026 \\
Upper 95\% & 0.5396 \\
Lower 95\% & -1.6729 \\
Sample size & 12 \\
Correlation & 0.6884 \\
Paired t test & -1.1274 \\
Degrees freedom & 11 \\
P value (2 tail) & 0.2836 \\
\hline
\end{tabular}


Table D-13. Assessment of intraobserver repeatability for the variable Axial CA R Initial.

\begin{tabular}{lc}
\hline \multicolumn{1}{c}{ Statistic } & Value \\
\hline Session Two (mean) & 60.0750 \\
Session One (mean) & 60.2833 \\
Mean Difference & -0.2083 \\
Standard Error & 0.5749 \\
Upper 95\% & 1.0570 \\
Lower 95\% & -1.4737 \\
Sample size & 12 \\
Correlation & 0.9200 \\
Paired t test & -0.3624 \\
Degrees freedom & 11 \\
P value (2 tail) & 0.7239 \\
\hline
\end{tabular}

Table D-14. Assessment of intraobserver repeatability for the variable Axial LACP L Final.

\begin{tabular}{lr}
\hline \multicolumn{1}{c}{ Statistic } & Value \\
\hline Session Two (mean) & 113.3750 \\
Session One (mean) & 110.0250 \\
Mean Difference & 3.3500 \\
Standard Error & 2.1356 \\
Upper 95\% & 8.0504 \\
Lower 95\% & -1.3504 \\
Sample size & 12 \\
Correlation & 0.9449 \\
Paired t test & 1.5687 \\
Degrees freedom & 11 \\
P value (2 tail) & 0.1450 \\
\hline
\end{tabular}


Table D-15. Assessment of intraobserver repeatability for the variable Axial LACP L Initial.

\begin{tabular}{lc}
\hline \multicolumn{1}{c}{ Statistic } & Value \\
\hline Session Two (mean) & 102.5000 \\
Session One (mean) & 100.5830 \\
Mean Difference & 1.9167 \\
Standard Error & 1.2719 \\
Upper 95\% & 4.7161 \\
Lower 95\% & -0.8827 \\
Sample size & 12 \\
Correlation & 0.9786 \\
Paired t test & 1.5069 \\
Degrees freedom & 11 \\
P value (2 tail) & 0.1600 \\
\hline
\end{tabular}

Table D-16. Assessment of intraobserver repeatability for the variable Axial LACP R Final.

\begin{tabular}{lr}
\hline \multicolumn{1}{c}{ Statistic } & Value \\
\hline Session Two (mean) & 106.4920 \\
Session One (mean) & 102.8670 \\
Mean Difference & 3.6250 \\
Standard Error & 2.1545 \\
Upper 95\% & 8.3670 \\
Lower 95\% & -1.1170 \\
Sample size & 12 \\
Correlation & 0.9181 \\
Paired t test & 1.6825 \\
Degrees freedom & 11 \\
P value (2 tail) & 0.1206 \\
\hline
\end{tabular}


Table D-17. Assessment of intraobserver repeatability for the variable Axial LACP R Initial.

\begin{tabular}{lr}
\hline \multicolumn{1}{c}{ Statistic } & Value \\
\hline Session Two (mean) & 105.2500 \\
Session One (mean) & 102.6670 \\
Mean Difference & 2.5833 \\
Standard Error & 1.5085 \\
Upper 95\% & 5.9034 \\
Lower 95\% & -0.7367 \\
Sample size & 12 \\
Correlation & 0.9431 \\
Paired t test & 1.7126 \\
Degrees freedom & 11 \\
P value (2 tail) & 0.1148 \\
\hline
\end{tabular}

Table D-18. Assessment of intraobserver repeatability for the variable Axial ML L Final.

\begin{tabular}{lc}
\hline \multicolumn{1}{c}{ Statistic } & Value \\
\hline Session Two (mean) & 18.2417 \\
Session One (mean) & 18.4583 \\
Mean Difference & -0.2167 \\
Standard Error & 0.1173 \\
Upper 95\% & 0.0415 \\
Lower 95\% & -0.4749 \\
Sample size & 12 \\
Correlation & 0.9804 \\
Paired t test & -1.8469 \\
Degrees freedom & 11 \\
P value (2 tail) & 0.0918 \\
\hline
\end{tabular}


Table D-19. Assessment of intraobserver repeatability for the variable Axial ML L Initial.

\begin{tabular}{lc}
\hline \multicolumn{1}{c}{ Statistic } & Value \\
\hline Session Two (mean) & 17.7250 \\
Session One (mean) & 17.6750 \\
Mean Difference & 0.0500 \\
Standard Error & 0.1469 \\
Upper 95\% & 0.3734 \\
Lower 95\% & -0.2734 \\
Sample size & 12 \\
Correlation & 0.9686 \\
Paired t test & 0.3403 \\
Degrees freedom & 11 \\
P value (2 tail) & 0.7401 \\
\hline
\end{tabular}

Table D-20. Assessment of intraobserver repeatability for the variable Axial ML R Final.

\begin{tabular}{lc}
\hline \multicolumn{1}{c}{ Statistic } & Value \\
\hline Session Two (mean) & 18.0667 \\
Session One (mean) & 18.0167 \\
Mean Difference & 0.0500 \\
Standard Error & 0.1721 \\
Upper 95\% & 0.4288 \\
Lower 95\% & -0.3288 \\
Sample size & 12 \\
Correlation & 0.9404 \\
Paired t test & 0.2905 \\
Degrees freedom & 11 \\
P value (2 tail) & 0.7768 \\
\hline
\end{tabular}


Table D-21. Assessment of intraobserver repeatability for the variable Axial ML R Initial.

\begin{tabular}{lc}
\hline \multicolumn{1}{c}{ Statistic } & Value \\
\hline Session Two (mean) & 17.6750 \\
Session One (mean) & 17.4500 \\
Mean Difference & 0.2250 \\
Standard Error & 0.1610 \\
Upper 95\% & 0.5794 \\
Lower 95\% & -0.1294 \\
Sample size & 12 \\
Correlation & 0.9383 \\
Paired t test & 1.3973 \\
Degrees freedom & 11 \\
P value (2 tail) & 0.1899 \\
\hline
\end{tabular}

Table D-22. Assessment of intraobserver repeatability for the variable Axial MLCP L Final.

\begin{tabular}{lc}
\hline \multicolumn{1}{c}{ Statistic } & Value \\
\hline & \\
Session Two (mean) & 49.4667 \\
Session One (mean) & 49.2833 \\
Mean Difference & 0.1833 \\
Standard Error & 0.2380 \\
Upper 95\% & 0.7072 \\
Lower 95\% & -0.3405 \\
Sample size & 12 \\
Correlation & 0.9495 \\
Paired t test & 0.7703 \\
Degrees freedom & 11 \\
P value (2 tail) & 0.4573 \\
\hline
\end{tabular}


Table D-23. Assessment of intraobserver repeatability for the variable Axial MLCP L Initial.

\begin{tabular}{lc}
\hline \multicolumn{1}{c}{ Statistic } & Value \\
\hline Session Two (mean) & \\
Session One (mean) & 47.7250 \\
Mean Difference & 47.8000 \\
Standard Error & -0.0750 \\
Upper 95\% & 0.1900 \\
Lower 95\% & 0.3431 \\
Sample size & -0.4931 \\
Correlation & 12 \\
Paired t test & 0.9641 \\
Degrees freedom & -0.3949 \\
P value (2 tail) & 11 \\
\hline
\end{tabular}

Table D-24. Assessment of intraobserver repeatability for the variable Axial MLCP R Final.

\begin{tabular}{lc}
\hline \multicolumn{1}{c}{ Statistic } & Value \\
\hline Session Two (mean) & 48.9583 \\
Session One (mean) & 49.6333 \\
Mean Difference & -0.6750 \\
Standard Error & 0.2529 \\
Upper 95\% & -0.1184 \\
Lower 95\% & -1.2316 \\
Sample size & 12 \\
Correlation & 0.9528 \\
Paired t test & -2.6690 \\
Degrees freedom & 11 \\
P value (2 tail) & 0.0218 \\
\hline
\end{tabular}


Table D-25. Assessment of intraobserver repeatability for the variable Axial MLCP R Initial.

\begin{tabular}{lc}
\hline \multicolumn{1}{c}{ Statistic } & Value \\
\hline Session Two (mean) & 47.6417 \\
Session One (mean) & 47.6083 \\
Mean Difference & 0.0333 \\
Standard Error & 0.1852 \\
Upper 95\% & 0.4409 \\
Lower 95\% & -0.3743 \\
Sample size & 12 \\
Correlation & 0.9717 \\
Paired t test & 0.1800 \\
Degrees freedom & 11 \\
P value (2 tail) & 0.8604 \\
\hline
\end{tabular}

Table D-26. Assessment of intraobserver repeatability for the variable Coronal LJS L Final.

\begin{tabular}{lc}
\hline \multicolumn{1}{c}{ Statistic } & Value \\
\hline Session Two (mean) & 2.4167 \\
Session One (mean) & 2.3000 \\
Mean Difference & 0.1167 \\
Standard Error & 0.0366 \\
Upper 95\% & 0.1972 \\
Lower 95\% & 0.0362 \\
Sample size & 12 \\
Correlation & 0.9915 \\
Paired t test & 3.1890 \\
Degrees freedom & 11 \\
P value (2 tail) & 0.0086 \\
\hline
\end{tabular}


Table D-27. Assessment of intraobserver repeatability for the variable Coronal LJS L Initial.

\begin{tabular}{lc}
\hline \multicolumn{1}{c}{ Statistic } & Value \\
\hline Session Two (mean) & \\
Session One (mean) & 2.5417 \\
Mean Difference & 2.5000 \\
Standard Error & 0.0417 \\
Upper 95\% & 0.0468 \\
Lower 95\% & 0.1447 \\
Sample size & -0.0613 \\
Correlation & 12 \\
Paired t test & 0.9848 \\
Degrees freedom & 0.8902 \\
P value (2 tail) & 11 \\
\hline
\end{tabular}

Table D-28. Assessment of intraobserver repeatability for the variable Coronal LJS R Final.

\begin{tabular}{lc}
\hline \multicolumn{1}{c}{ Statistic } & Value \\
\hline & \\
Session Two (mean) & 2.5583 \\
Session One (mean) & 2.5750 \\
Mean Difference & -0.0167 \\
Standard Error & 0.0548 \\
Upper 95\% & 0.1040 \\
Lower 95\% & -0.1373 \\
Sample size & 12 \\
Correlation & 0.9692 \\
Paired t test & -0.3040 \\
Degrees freedom & 11 \\
P value (2 tail) & 0.7668 \\
\hline
\end{tabular}


Table D-29. Assessment of intraobserver repeatability for the variable Coronal LJS R Initial.

\begin{tabular}{lc}
\hline \multicolumn{1}{c}{ Statistic } & Value \\
\hline Session Two (mean) & \\
Session One (mean) & 2.1917 \\
Mean Difference & 2.1750 \\
Standard Error & 0.0167 \\
Upper 95\% & 0.0297 \\
Lower 95\% & 0.0821 \\
Sample size & -0.0488 \\
Correlation & 12 \\
Paired t test & 0.9889 \\
Degrees freedom & 0.5606 \\
P value (2 tail) & 11 \\
\hline
\end{tabular}

Table D-30. Assessment of intraobserver repeatability for the variable Coronal MJS L Final.

\begin{tabular}{lc}
\hline \multicolumn{1}{c}{ Statistic } & Value \\
\hline Session Two (mean) & \\
Session One (mean) & 2.3000 \\
Mean Difference & 2.1917 \\
Standard Error & 0.1083 \\
Upper 95\% & 0.0633 \\
Lower 95\% & 0.2477 \\
Sample size & -0.0310 \\
Correlation & 12 \\
Paired t test & 0.9413 \\
Degrees freedom & 1.7110 \\
P value (2 tail) & 11 \\
\hline
\end{tabular}


Table D-31. Assessment of intraobserver repeatability for the variable Coronal MJS L Initial.

\begin{tabular}{lc}
\hline \multicolumn{1}{c}{ Statistic } & Value \\
\hline Session Two (mean) & 2.4333 \\
Session One (mean) & 2.4750 \\
Mean Difference & -0.0417 \\
Standard Error & 0.0484 \\
Upper 95\% & 0.0649 \\
Lower 95\% & -0.1482 \\
Sample size & 12 \\
Correlation & 0.9678 \\
Paired t test & -0.8610 \\
Degrees freedom & 11 \\
P value (2 tail) & 0.4076 \\
\hline
\end{tabular}

Table D-32. Assessment of intraobserver repeatability for the variable Coronal MJS R Final.

\begin{tabular}{lc}
\hline \multicolumn{1}{c}{ Statistic } & Value \\
\hline Session Two (mean) & 2.3000 \\
Session One (mean) & 2.2750 \\
Mean Difference & 0.0250 \\
Standard Error & 0.0509 \\
Upper 95\% & 0.1371 \\
Lower 95\% & -0.0871 \\
Sample size & 12 \\
Correlation & 0.9747 \\
Paired t test & 0.4908 \\
Degrees freedom & 11 \\
P value (2 tail) & 0.6332 \\
\hline
\end{tabular}


Table D-33. Assessment of intraobserver repeatability for the variable Coronal MJS R Initial.

\begin{tabular}{lc}
\hline \multicolumn{1}{c}{ Statistic } & Value \\
\hline Session Two (mean) & 2.0750 \\
Session One (mean) & 2.1083 \\
Mean Difference & -0.0333 \\
Standard Error & 0.0376 \\
Upper 95\% & 0.0494 \\
Lower 95\% & -0.1161 \\
Sample size & 12 \\
Correlation & 0.9926 \\
Paired t test & -0.8864 \\
Degrees freedom & 11 \\
P value (2 tail) & 0.3944 \\
\hline
\end{tabular}

Table D-34. Assessment of intraobserver repeatability for the variable Coronal SJS L Final.

\begin{tabular}{lc}
\hline \multicolumn{1}{c}{ Statistic } & Value \\
\hline Session Two (mean) & 2.0667 \\
Session One (mean) & 2.0083 \\
Mean Difference & 0.0583 \\
Standard Error & 0.0583 \\
Upper 95\% & 0.1867 \\
Lower 95\% & -0.0701 \\
Sample size & 12 \\
Correlation & 0.9749 \\
Paired t test & 1.0000 \\
Degrees freedom & 11 \\
P value (2 tail) & 0.3388 \\
\hline
\end{tabular}


Table D-35. Assessment of intraobserver repeatability for the variable Coronal SJS L Initial.

\begin{tabular}{lc}
\hline \multicolumn{1}{c}{ Statistic } & Value \\
\hline Session Two (mean) & 2.1000 \\
Session One (mean) & 2.0750 \\
Mean Difference & 0.0250 \\
Standard Error & 0.0566 \\
Upper 95\% & 0.1495 \\
Lower 95\% & -0.0995 \\
Sample size & 12 \\
Correlation & 0.9863 \\
Paired t test & 0.4419 \\
Degrees freedom & 11 \\
P value (2 tail) & 0.6671 \\
\hline
\end{tabular}

Table D-36. Assessment of intraobserver repeatability for the variable Coronal SJS R Final.

\begin{tabular}{lc}
\hline \multicolumn{1}{c}{ Statistic } & Value \\
\hline Session Two (mean) & 1.8667 \\
Session One (mean) & 1.8750 \\
Mean Difference & -0.0083 \\
Standard Error & 0.0633 \\
Upper 95\% & 0.1310 \\
Lower 95\% & -0.1477 \\
Sample size & 12 \\
Correlation & 0.9681 \\
Paired t test & -0.1316 \\
Degrees freedom & 11 \\
P value (2 tail) & 0.8977 \\
\hline
\end{tabular}


Table D-37. Assessment of intraobserver repeatability for the variable Coronal SJS R Initial.

\begin{tabular}{lc}
\hline \multicolumn{1}{c}{ Statistic } & Value \\
\hline Session Two (mean) & \\
Session One (mean) & 2.1167 \\
Mean Difference & 2.0583 \\
Standard Error & 0.0583 \\
Upper 95\% & 0.0435 \\
Lower 95\% & 0.1540 \\
Sample size & -0.0373 \\
Correlation & 12 \\
Paired t test & 0.9811 \\
Degrees freedom & 1.3426 \\
P value (2 tail) & 11 \\
\hline
\end{tabular}

Table D-38. Assessment of intraobserver repeatability for the variable Mesial Molar Relation Final.

\begin{tabular}{lc}
\hline \multicolumn{1}{c}{ Statistic } & Value \\
\hline Session Two (mean) & -0.6000 \\
Session One (mean) & -0.8750 \\
Mean Difference & 0.2750 \\
Standard Error & 0.1431 \\
Upper 95\% & 0.5899 \\
Lower 95\% & -0.0399 \\
Sample size & 12 \\
Correlation & 0.8971 \\
Paired t test & 1.9219 \\
Degrees freedom & 11 \\
P value (2 tail) & 0.0809 \\
\hline
\end{tabular}


Table D-39. Assessment of intraobserver repeatability for the variable Mesial Molar Relation Initial.

\begin{tabular}{lc}
\hline \multicolumn{1}{c}{ Statistic } & Value \\
\hline Session Two (mean) & 1.3417 \\
Session One (mean) & 0.9917 \\
Mean Difference & 0.3500 \\
Standard Error & 0.1258 \\
Upper 95\% & 0.6270 \\
Lower 95\% & 0.0731 \\
Sample size & 12 \\
Correlation & 0.9211 \\
Paired t test & 2.7815 \\
Degrees freedom & 11 \\
P value (2 tail) & 0.0179 \\
\hline
\end{tabular}

Table D-40. Assessment of intraobserver repeatability for the variable Overbite Final.

\begin{tabular}{lc}
\hline \multicolumn{1}{c}{ Statistic } & Value \\
\hline Session Two (mean) & 1.2417 \\
Session One (mean) & 0.9583 \\
Mean Difference & 0.2833 \\
Standard Error & 0.1260 \\
Upper 95\% & 0.5607 \\
Lower 95\% & 0.0059 \\
Sample size & 12 \\
Correlation & 0.9547 \\
Paired t test & 2.2481 \\
Degrees freedom & 11 \\
P value (2 tail) & 0.0460 \\
\hline
\end{tabular}


Table D-41. Assessment of intraobserver repeatability for the variable Overbite Initial.

\begin{tabular}{lc}
\hline \multicolumn{1}{c}{ Statistic } & Value \\
\hline Session Two (mean) & \\
Session One (mean) & 4.2500 \\
Mean Difference & 4.1083 \\
Standard Error & 0.1417 \\
Upper 95\% & 0.1340 \\
Lower 95\% & 0.4365 \\
Sample size & -0.1532 \\
Correlation & 12 \\
Paired t test & 0.9625 \\
Degrees freedom & 1.0574 \\
P value (2 tail) & 11 \\
\hline
\end{tabular}

Table D-42. Assessment of intraobserver repeatability for the variable Overjet Initial.

\begin{tabular}{lc}
\hline \multicolumn{1}{c}{ Statistic } & Value \\
\hline Session Two (mean) & 5.6083 \\
Session One (mean) & 5.7750 \\
Mean Difference & -0.1667 \\
Standard Error & 0.0882 \\
Upper 95\% & 0.0274 \\
Lower 95\% & -0.3608 \\
Sample size & 12 \\
Correlation & 0.9805 \\
Paired t test & -1.8898 \\
Degrees freedom & 11 \\
P value (2 tail) & 0.0854 \\
\hline
\end{tabular}


Table D-43. Assessment of intraobserver repeatability for the variable Sagittal AAE L Final.

\begin{tabular}{lc}
\hline \multicolumn{1}{c}{ Statistic } & Value \\
\hline Session Two (mean) & 100.9080 \\
Session One (mean) & 106.1670 \\
Mean Difference & -5.2583 \\
Standard Error & 2.4645 \\
Upper 95\% & 0.1660 \\
Lower 95\% & -10.6830 \\
Sample size & 12 \\
Correlation & 0.8936 \\
Paired t test & -2.1336 \\
Degrees freedom & 11 \\
P value (2 tail) & 0.0562 \\
\hline
\end{tabular}

Table D-44. Assessment of intraobserver repeatability for the variable Sagittal AAE L Initial.

\begin{tabular}{lr}
\hline \multicolumn{1}{c}{ Statistic } & Value \\
\hline Session Two (mean) & 154.5500 \\
Session One (mean) & 156.1170 \\
Mean Difference & -1.5667 \\
Standard Error & 4.1265 \\
Upper 95\% & 7.5157 \\
Lower 95\% & -10.6490 \\
Sample size & 12 \\
Correlation & 0.9978 \\
Paired t test & -0.3797 \\
Degrees freedom & 11 \\
P value (2 tail) & 0.7114 \\
\hline
\end{tabular}


Table D-45. Assessment of intraobserver repeatability for the variable Sagittal AAE R Final.

\begin{tabular}{lc}
\hline \multicolumn{1}{c}{ Statistic } & Value \\
\hline Session Two (mean) & 107.9330 \\
Session One (mean) & 113.6170 \\
Mean Difference & -5.6833 \\
Standard Error & 2.8928 \\
Upper 95\% & 0.6837 \\
Lower 95\% & -12.0500 \\
Sample size & 12 \\
Correlation & 0.9299 \\
Paired t test & -1.9647 \\
Degrees freedom & 11 \\
P value (2 tail) & 0.0752 \\
\hline
\end{tabular}

Table D-46. Assessment of intraobserver repeatability for the variable Sagittal AAE R Initial.

\begin{tabular}{lr}
\hline \multicolumn{1}{c}{ Statistic } & Value \\
\hline Session Two (mean) & 103.2250 \\
Session One (mean) & 104.0330 \\
Mean Difference & -0.8083 \\
Standard Error & 2.6012 \\
Upper 95\% & 4.9170 \\
Lower 95\% & -6.5336 \\
Sample size & 12 \\
Correlation & 0.8985 \\
Paired t test & -0.3108 \\
Degrees freedom & 11 \\
P value (2 tail) & 0.7618 \\
\hline
\end{tabular}


Table D-47. Assessment of intraobserver repeatability for the variable Sagittal ACP L Final.

\begin{tabular}{lc}
\hline \multicolumn{1}{c}{ Statistic } & Value \\
\hline Session Two (mean) & 46.8250 \\
Session One (mean) & 49.1167 \\
Mean Difference & -2.2917 \\
Standard Error & 1.5544 \\
Upper 95\% & 1.1296 \\
Lower 95\% & -5.7130 \\
Sample size & 12 \\
Correlation & 0.9129 \\
Paired t test & -1.4743 \\
Degrees freedom & 11 \\
P value (2 tail) & 0.1684 \\
\hline
\end{tabular}

Table D-48. Assessment of intraobserver repeatability for the variable Sagittal ACP L Initial.

\begin{tabular}{lc}
\hline \multicolumn{1}{c}{ Statistic } & Value \\
\hline & \\
Session Two (mean) & 46.3250 \\
Session One (mean) & 48.7583 \\
Mean Difference & -2.4333 \\
Standard Error & 1.3444 \\
Upper 95\% & 0.5256 \\
Lower 95\% & -5.3923 \\
Sample size & 12 \\
Correlation & 0.9499 \\
Paired t test & -1.8100 \\
Degrees freedom & 11 \\
P value (2 tail) & 0.0977 \\
\hline
\end{tabular}


Table D-49. Assessment of intraobserver repeatability for the variable Sagittal ACP R Final.

\begin{tabular}{lc}
\hline \multicolumn{1}{c}{ Statistic } & Value \\
\hline Session Two (mean) & 50.0083 \\
Session One (mean) & 50.5583 \\
Mean Difference & -0.5500 \\
Standard Error & 1.5393 \\
Upper 95\% & 2.8380 \\
Lower 95\% & -3.9380 \\
Sample size & 12 \\
Correlation & 0.8981 \\
Paired t test & -0.3573 \\
Degrees freedom & 11 \\
P value (2 tail) & 0.7276 \\
\hline
\end{tabular}

Table D-50. Assessment of intraobserver repeatability for the variable Sagittal ACP R Initial.

\begin{tabular}{lc}
\hline \multicolumn{1}{c}{ Statistic } & Value \\
\hline Session Two (mean) & 48.2000 \\
Session One (mean) & 47.5833 \\
Mean Difference & 0.6167 \\
Standard Error & 1.2340 \\
Upper 95\% & 3.3326 \\
Lower 95\% & -2.0993 \\
Sample size & 12 \\
Correlation & 0.8974 \\
Paired t test & 0.0000 \\
Degrees freedom & 11 \\
P value (2 tail) & 0.6271 \\
\hline
\end{tabular}


Table D-51. Assessment of intraobserver repeatability for the variable Sagittal AGF L Final.

\begin{tabular}{lc}
\hline \multicolumn{1}{c}{ Statistic } & Value \\
\hline Session Two (mean) & 82.4500 \\
Session One (mean) & 81.7500 \\
Mean Difference & 0.7000 \\
Standard Error & 2.4684 \\
Upper 95\% & 6.1329 \\
Lower 95\% & -4.7329 \\
Sample size & 12 \\
Correlation & 0.8619 \\
Paired t test & 0.2836 \\
Degrees freedom & 11 \\
P value (2 tail) & 0.7820 \\
\hline
\end{tabular}

Table D-52. Assessment of intraobserver repeatability for the variable Sagittal AGF L Initial.

\begin{tabular}{lc}
\hline \multicolumn{1}{c}{ Statistic } & Value \\
\hline Session Two (mean) & 77.7167 \\
Session One (mean) & 77.6083 \\
Mean Difference & 0.1083 \\
Standard Error & 1.5888 \\
Upper 95\% & 3.6053 \\
Lower 95\% & -3.3886 \\
Sample size & 12 \\
Correlation & 0.9441 \\
Paired t test & 0.0682 \\
Degrees freedom & 11 \\
P value (2 tail) & 0.9469 \\
\hline
\end{tabular}


Table D-53. Assessment of intraobserver repeatability for the variable Sagittal AGF R Final.

\begin{tabular}{lc}
\hline \multicolumn{1}{c}{ Statistic } & Value \\
\hline Session Two (mean) & \\
Session One (mean) & 85.3500 \\
Mean Difference & 86.4083 \\
Standard Error & -1.0583 \\
Upper 95\% & 3.3157 \\
Lower 95\% & 6.2394 \\
Sample size & -8.3561 \\
Correlation & 12 \\
Paired t test & 0.7871 \\
Degrees freedom & -0.3192 \\
P value (2 tail) & 11 \\
\hline
\end{tabular}

Table D-54. Assessment of intraobserver repeatability for the variable Sagittal AGF R Initial.

\begin{tabular}{lc}
\hline \multicolumn{1}{c}{ Statistic } & Value \\
\hline & \\
Session Two (mean) & 80.0750 \\
Session One (mean) & 78.2083 \\
Mean Difference & 1.8667 \\
Standard Error & 2.5385 \\
Upper 95\% & 7.4539 \\
Lower 95\% & -3.7205 \\
Sample size & 12 \\
Correlation & 0.9201 \\
Paired t test & 0.7353 \\
Degrees freedom & 11 \\
P value (2 tail) & 0.4775 \\
\hline
\end{tabular}


Table D-55. Assessment of intraobserver repeatability for the variable Sagittal AJS L Final.

\begin{tabular}{lc}
\hline \multicolumn{1}{c}{ Statistic } & Value \\
\hline Session Two (mean) & 1.2083 \\
Session One (mean) & 1.1333 \\
Mean Difference & 0.0750 \\
Standard Error & 0.0279 \\
Upper 95\% & 0.1363 \\
Lower 95\% & 0.0137 \\
Sample size & 12 \\
Correlation & 0.9336 \\
Paired t test & 2.6915 \\
Degrees freedom & 11 \\
P value (2 tail) & 0.0210 \\
\hline
\end{tabular}

Table D-56. Assessment of intraobserver repeatability for the variable Sagittal AJS L Initial.

\begin{tabular}{lc}
\hline \multicolumn{1}{c}{ Statistic } & Value \\
\hline Session Two (mean) & 1.3333 \\
Session One (mean) & 1.3333 \\
Mean Difference & 0.0000 \\
Standard Error & 0.0426 \\
Upper 95\% & 0.0939 \\
Lower 95\% & -0.0939 \\
Sample size & 12 \\
Correlation & 0.9669 \\
Paired t test & 0.0000 \\
Degrees freedom & 11 \\
P value (2 tail) & 1.0000 \\
\hline
\end{tabular}


Table D-57. Assessment of intraobserver repeatability for the variable Sagittal AJS R Final.

\begin{tabular}{lc}
\hline \multicolumn{1}{c}{ Statistic } & Value \\
\hline Session Two (mean) & 1.7500 \\
Session One (mean) & 1.6917 \\
Mean Difference & 0.0583 \\
Standard Error & 0.0379 \\
Upper 95\% & 0.1417 \\
Lower 95\% & -0.0250 \\
Sample size & 12 \\
Correlation & 0.9801 \\
Paired t test & 1.5409 \\
Degrees freedom & 11 \\
P value (2 tail) & 0.1516 \\
\hline
\end{tabular}

Table D-58. Assessment of intraobserver repeatability for the variable Sagittal AJS R Initial.

\begin{tabular}{lc}
\hline \multicolumn{1}{c}{ Statistic } & Value \\
\hline Session Two (mean) & 1.4083 \\
Session One (mean) & 1.3667 \\
Mean Difference & 0.0417 \\
Standard Error & 0.0468 \\
Upper 95\% & 0.1447 \\
Lower 95\% & -0.0613 \\
Sample size & 12 \\
Correlation & 0.9627 \\
Paired t test & 0.8902 \\
Degrees freedom & 11 \\
P value (2 tail) & 0.3924 \\
\hline
\end{tabular}


Table D-59. Assessment of intraobserver repeatability for the variable Sagittal ATA L Final.

\begin{tabular}{lc}
\hline \multicolumn{1}{c}{ Statistic } & Value \\
\hline Session Two (mean) & 36.6000 \\
Session One (mean) & 39.1333 \\
Mean Difference & -2.5333 \\
Standard Error & 1.8710 \\
Upper 95\% & 1.5846 \\
Lower 95\% & -6.6513 \\
Sample size & 12 \\
Correlation & 0.4635 \\
Paired t test & -1.3540 \\
Degrees freedom & 11 \\
P value (2 tail) & 0.2029 \\
\hline
\end{tabular}

Table D-60. Assessment of intraobserver repeatability for the variable Sagittal ATA L Initial.

\begin{tabular}{lc}
\hline \multicolumn{1}{c}{ Statistic } & Value \\
\hline Session Two (mean) & 39.2750 \\
Session One (mean) & 39.6500 \\
Mean Difference & -0.3750 \\
Standard Error & 0.6965 \\
Upper 95\% & 1.1581 \\
Lower 95\% & -1.9081 \\
Sample size & 12 \\
Correlation & 0.8929 \\
Paired t test & -0.5384 \\
Degrees freedom & 11 \\
P value (2 tail) & 0.6010 \\
\hline
\end{tabular}


Table D-61. Assessment of intraobserver repeatability for the variable Sagittal ATA R Final.

\begin{tabular}{lc}
\hline \multicolumn{1}{c}{ Statistic } & Value \\
\hline Session Two (mean) & 38.4167 \\
Session One (mean) & 38.0917 \\
Mean Difference & 0.3250 \\
Standard Error & 0.2769 \\
Upper 95\% & 0.9345 \\
Lower 95\% & -0.2845 \\
Sample size & 12 \\
Correlation & 0.9845 \\
Paired t test & 1.1736 \\
Degrees freedom & 11 \\
P value (2 tail) & 0.2653 \\
\hline
\end{tabular}

Table D-62. Assessment of intraobserver repeatability for the variable Sagittal ATA R Initial.

\begin{tabular}{lc}
\hline \multicolumn{1}{c}{ Statistic } & Value \\
\hline & \\
Session Two (mean) & 38.9083 \\
Session One (mean) & 38.5833 \\
Mean Difference & 0.3250 \\
Standard Error & 0.4068 \\
Upper 95\% & 1.2203 \\
Lower 95\% & -0.5703 \\
Sample size & 12 \\
Correlation & 0.9858 \\
Paired t test & 0.7989 \\
Degrees freedom & 11 \\
P value (2 tail) & 0.4412 \\
\hline
\end{tabular}


Table D-63. Assessment of intraobserver repeatability for the variable Sagittal CH L Final.

\begin{tabular}{lc}
\hline \multicolumn{1}{c}{ Statistic } & Value \\
\hline Session Two (mean) & 17.5333 \\
Session One (mean) & 17.5750 \\
Mean Difference & -0.0417 \\
Standard Error & 0.0723 \\
Upper 95\% & 0.1174 \\
Lower 95\% & -0.2007 \\
Sample size & 12 \\
Correlation & 0.9954 \\
Paired t test & -0.5767 \\
Degrees freedom & 11 \\
P value (2 tail) & 0.5758 \\
\hline
\end{tabular}

Table D-64. Assessment of intraobserver repeatability for the variable Sagittal CH L Initial.

\begin{tabular}{lc}
\hline \multicolumn{1}{c}{ Statistic } & Value \\
\hline & \\
Session Two (mean) & 15.7083 \\
Session One (mean) & 15.6750 \\
Mean Difference & 0.0333 \\
Standard Error & 0.0732 \\
Upper 95\% & 0.1944 \\
Lower 95\% & -0.1277 \\
Sample size & 12 \\
Correlation & 0.9953 \\
Paired t test & 0.4556 \\
Degrees freedom & 11 \\
P value (2 tail) & 0.6576 \\
\hline
\end{tabular}


Table D-65. Assessment of intraobserver repeatability for the variable Sagittal CH R Final.

\begin{tabular}{lc}
\hline \multicolumn{1}{c}{ Statistic } & Value \\
\hline Session Two (mean) & 16.8750 \\
Session One (mean) & 16.7000 \\
Mean Difference & 0.1750 \\
Standard Error & 0.1142 \\
Upper 95\% & 0.4264 \\
Lower 95\% & -0.0764 \\
Sample size & 12 \\
Correlation & 0.9843 \\
Paired t test & 1.5320 \\
Degrees freedom & 11 \\
P value (2 tail) & 0.1538 \\
\hline
\end{tabular}

Table D-66. Assessment of intraobserver repeatability for the variable Sagittal CH R Initial.

\begin{tabular}{lc}
\hline \multicolumn{1}{c}{ Statistic } & Value \\
\hline Session Two (mean) & 15.4333 \\
Session One (mean) & 15.2917 \\
Mean Difference & 0.1417 \\
Standard Error & 0.0892 \\
Upper 95\% & 0.3379 \\
Lower 95\% & -0.0546 \\
Sample size & 12 \\
Correlation & 0.9938 \\
Paired t test & 1.5890 \\
Degrees freedom & 11 \\
P value (2 tail) & 0.1404 \\
\hline
\end{tabular}


Table D-67. Assessment of intraobserver repeatability for the variable Sagittal CHA L Final.

\begin{tabular}{lr}
\hline \multicolumn{1}{c}{ Statistic } & Value \\
\hline Session Two (mean) & 140.3580 \\
Session One (mean) & 140.2170 \\
Mean Difference & 0.1417 \\
Standard Error & 0.5062 \\
Upper 95\% & 1.2559 \\
Lower 95\% & -0.9726 \\
Sample size & 12 \\
Correlation & 0.9820 \\
Paired t test & 0.2798 \\
Degrees freedom & 11 \\
P value (2 tail) & 0.7848 \\
\hline
\end{tabular}

Table D-68. Assessment of intraobserver repeatability for the variable Sagittal CHA L Initial.

\begin{tabular}{lc}
\hline \multicolumn{1}{c}{ Statistic } & Value \\
\hline Session Two (mean) & 146.5000 \\
Session One (mean) & 147.7080 \\
Mean Difference & -1.2083 \\
Standard Error & 0.9410 \\
Upper 95\% & 0.8628 \\
Lower 95\% & -3.2795 \\
Sample size & 12 \\
Correlation & 0.8829 \\
Paired t test & -1.2841 \\
Degrees freedom & 11 \\
P value (2 tail) & 0.2255 \\
\hline
\end{tabular}


Table D-69. Assessment of intraobserver repeatability for the variable Sagittal CHA R Final.

\begin{tabular}{lc}
\hline \multicolumn{1}{c}{ Statistic } & Value \\
\hline Session Two (mean) & 140.9420 \\
Session One (mean) & 141.7000 \\
Mean Difference & -0.7583 \\
Standard Error & 0.5803 \\
Upper 95\% & 0.5189 \\
Lower 95\% & -2.0355 \\
Sample size & 12 \\
Correlation & 0.9833 \\
Paired t test & -1.3068 \\
Degrees freedom & 11 \\
P value (2 tail) & 0.2179 \\
\hline
\end{tabular}

Table D-70. Assessment of intraobserver repeatability for the variable Sagittal CHA R Initial.

\begin{tabular}{lr}
\hline \multicolumn{1}{c}{ Statistic } & Value \\
\hline Session Two (mean) & 144.4080 \\
Session One (mean) & 145.0750 \\
Mean Difference & -0.6667 \\
Standard Error & 0.5920 \\
Upper 95\% & 0.6364 \\
Lower 95\% & -1.9697 \\
Sample size & 12 \\
Correlation & 0.9622 \\
Paired t test & -1.1261 \\
Degrees freedom & 11 \\
P value (2 tail) & 0.2841 \\
\hline
\end{tabular}


Table D-71. Assessment of intraobserver repeatability for the variable Sagittal CHH L Final.

\begin{tabular}{lc}
\hline \multicolumn{1}{c}{ Statistic } & Value \\
\hline Session Two (mean) & 6.4250 \\
Session One (mean) & 6.4083 \\
Mean Difference & 0.0167 \\
Standard Error & 0.0520 \\
Upper 95\% & 0.1311 \\
Lower 95\% & -0.0977 \\
Sample size & 12 \\
Correlation & 0.9820 \\
Paired t test & 0.3206 \\
Degrees freedom & 11 \\
P value (2 tail) & 0.7545 \\
\hline
\end{tabular}

Table D-72. Assessment of intraobserver repeatability for the variable Sagittal CHH L Initial.

\begin{tabular}{lc}
\hline \multicolumn{1}{c}{ Statistic } & Value \\
\hline & \\
Session Two (mean) & 5.9917 \\
Session One (mean) & 6.0333 \\
Mean Difference & -0.0417 \\
Standard Error & 0.0645 \\
Upper 95\% & 0.1003 \\
Lower 95\% & -0.1836 \\
Sample size & 12 \\
Correlation & 0.9609 \\
Paired t test & -0.6460 \\
Degrees freedom & 11 \\
P value (2 tail) & 0.5315 \\
\hline
\end{tabular}


Table D-73. Assessment of intraobserver repeatability for the variable Sagittal CHH R Final.

\begin{tabular}{lc}
\hline \multicolumn{1}{c}{ Statistic } & Value \\
\hline Session Two (mean) & 6.5667 \\
Session One (mean) & 6.5333 \\
Mean Difference & 0.0333 \\
Standard Error & 0.0700 \\
Upper 95\% & 0.1874 \\
Lower 95\% & -0.1207 \\
Sample size & 12 \\
Correlation & 0.9421 \\
Paired t test & 0.4762 \\
Degrees freedom & 11 \\
P value (2 tail) & 0.6432 \\
\hline
\end{tabular}

Table D-74. Assessment of intraobserver repeatability for the variable Sagittal CHH R Initial.

\begin{tabular}{lc}
\hline \multicolumn{1}{c}{ Statistic } & Value \\
\hline & \\
Session Two (mean) & 6.3750 \\
Session One (mean) & 6.3500 \\
Mean Difference & 0.0250 \\
Standard Error & 0.0730 \\
Upper 95\% & 0.1856 \\
Lower 95\% & -0.1356 \\
Sample size & 12 \\
Correlation & 0.8969 \\
Paired t test & 0.3427 \\
Degrees freedom & 11 \\
P value (2 tail) & 0.7383 \\
\hline
\end{tabular}


Table D-75. Assessment of intraobserver repeatability for the variable Sagittal CHW L Final.

\begin{tabular}{lc}
\hline \multicolumn{1}{c}{ Statistic } & Value \\
\hline Session Two (mean) & \\
Session One (mean) & 8.9333 \\
Mean Difference & 8.9500 \\
Standard Error & -0.0167 \\
Upper 95\% & 0.0613 \\
Lower 95\% & 0.1183 \\
Sample size & -0.1517 \\
Correlation & 12 \\
Paired t test & 0.9850 \\
Degrees freedom & -0.2717 \\
P value (2 tail) & 11 \\
\hline
\end{tabular}

Table D-76. Assessment of intraobserver repeatability for the variable Sagittal CHW L Initial.

\begin{tabular}{lc}
\hline \multicolumn{1}{c}{ Statistic } & Value \\
\hline & \\
Session Two (mean) & 9.0833 \\
Session One (mean) & 9.0167 \\
Mean Difference & 0.0667 \\
Standard Error & 0.0689 \\
Upper 95\% & 0.2183 \\
Lower 95\% & -0.0850 \\
Sample size & 12 \\
Correlation & 0.9767 \\
Paired t test & 0.9676 \\
Degrees freedom & 11 \\
P value (2 tail) & 0.3541 \\
\hline
\end{tabular}


Table D-77. Assessment of intraobserver repeatability for the variable Sagittal CHW R Final.

\begin{tabular}{lc}
\hline \multicolumn{1}{c}{ Statistic } & Value \\
\hline & \\
Session Two (mean) & 8.9167 \\
Session One (mean) & 8.9833 \\
Mean Difference & -0.0667 \\
Standard Error & 0.0527 \\
Upper 95\% & 0.0493 \\
Lower 95\% & -0.1827 \\
Sample size & 12 \\
Correlation & 0.9918 \\
Paired t test & -1.2649 \\
Degrees freedom & 11 \\
P value (2 tail) & 0.2320 \\
\hline
\end{tabular}

Table D-78. Assessment of intraobserver repeatability for the variable Sagittal CHW R Initial.

\begin{tabular}{lc}
\hline \multicolumn{1}{c}{ Statistic } & Value \\
\hline & \\
Session Two (mean) & 8.9417 \\
Session One (mean) & 8.9583 \\
Mean Difference & -0.0167 \\
Standard Error & 0.0649 \\
Upper 95\% & 0.1263 \\
Lower 95\% & -0.1596 \\
Sample size & 12 \\
Correlation & 0.9772 \\
Paired t test & -0.2567 \\
Degrees freedom & 11 \\
P value (2 tail) & 0.8022 \\
\hline
\end{tabular}


Table D-79. Assessment of intraobserver repeatability for the variable Sagittal EAM-AE L Final.

\begin{tabular}{lc}
\hline \multicolumn{1}{c}{ Statistic } & Value \\
\hline Session Two (mean) & 24.1917 \\
Session One (mean) & 24.2333 \\
Mean Difference & -0.0417 \\
Standard Error & 0.1276 \\
Upper 95\% & 0.2392 \\
Lower 95\% & -0.3225 \\
Sample size & 12 \\
Correlation & 0.9024 \\
Paired t test & -0.3265 \\
Degrees freedom & 11 \\
P value (2 tail) & 0.7501 \\
\hline
\end{tabular}

Table D-80. Assessment of intraobserver repeatability for the variable Sagittal EAM-AE L Initial.

\begin{tabular}{lc}
\hline \multicolumn{1}{c}{ Statistic } & Value \\
\hline Session Two (mean) & 23.3417 \\
Session One (mean) & 23.3833 \\
Mean Difference & -0.0417 \\
Standard Error & 0.1334 \\
Upper 95\% & 0.2520 \\
Lower 95\% & -0.3353 \\
Sample size & 12 \\
Correlation & 0.9537 \\
Paired t test & -0.3123 \\
Degrees freedom & 11 \\
P value (2 tail) & 0.7606 \\
\hline
\end{tabular}


Table D-81. Assessment of intraobserver repeatability for the variable Sagittal EAM-AE R Final.

\begin{tabular}{lc}
\hline \multicolumn{1}{c}{ Statistic } & Value \\
\hline Session Two (mean) & \\
Session One (mean) & 25.1750 \\
Mean Difference & 25.1583 \\
Standard Error & 0.0167 \\
Upper 95\% & 0.1107 \\
Lower 95\% & 0.2603 \\
Sample size & -0.2269 \\
Correlation & 12 \\
Paired t test & 0.9815 \\
Degrees freedom & 0.1506 \\
P value (2 tail) & 11 \\
\hline
\end{tabular}

Table D-82. Assessment of intraobserver repeatability for the variable Sagittal EAM-AE R Initial.

\begin{tabular}{lc}
\hline \multicolumn{1}{c}{ Statistic } & Value \\
\hline Session Two (mean) & 24.1667 \\
Session One (mean) & 24.3083 \\
Mean Difference & -0.1417 \\
Standard Error & 0.1118 \\
Upper 95\% & 0.1044 \\
Lower 95\% & -0.3877 \\
Sample size & 12 \\
Correlation & 0.9851 \\
Paired t test & -1.2674 \\
Degrees freedom & 11 \\
P value (2 tail) & 0.2312 \\
\hline
\end{tabular}


Table D-83. Assessment of intraobserver repeatability for the variable Sagittal EAM-C L Final.

\begin{tabular}{lc}
\hline \multicolumn{1}{c}{ Statistic } & Value \\
\hline Session Two (mean) & 14.1083 \\
Session One (mean) & 14.0000 \\
Mean Difference & 0.1083 \\
Standard Error & 0.0596 \\
Upper 95\% & 0.2396 \\
Lower 95\% & -0.0229 \\
Sample size & 12 \\
Correlation & 0.9965 \\
Paired t test & 1.8171 \\
Degrees freedom & 11 \\
P value (2 tail) & 0.0965 \\
\hline
\end{tabular}

Table D-84. Assessment of intraobserver repeatability for the variable Sagittal EAM-C L Initial.

\begin{tabular}{lc}
\hline \multicolumn{1}{c}{ Statistic } & Value \\
\hline Session Two (mean) & 13.0250 \\
Session One (mean) & 12.9417 \\
Mean Difference & 0.0833 \\
Standard Error & 0.0787 \\
Upper 95\% & 0.2565 \\
Lower 95\% & -0.0898 \\
Sample size & 12 \\
Correlation & 0.9666 \\
Paired t test & 1.0595 \\
Degrees freedom & 11 \\
P value (2 tail) & 0.3121 \\
\hline
\end{tabular}


Table D-85. Assessment of intraobserver repeatability for the variable Sagittal EAM-C R Final.

\begin{tabular}{lc}
\hline \multicolumn{1}{c}{ Statistic } & Value \\
\hline Session Two (mean) & 13.7083 \\
Session One (mean) & 13.6000 \\
Mean Difference & 0.1083 \\
Standard Error & 0.0783 \\
Upper 95\% & 0.2807 \\
Lower 95\% & -0.0640 \\
Sample size & 12 \\
Correlation & 0.9861 \\
Paired t test & 1.3837 \\
Degrees freedom & 11 \\
P value (2 tail) & 0.1939 \\
\hline
\end{tabular}

Table D-86. Assessment of intraobserver repeatability for the variable Sagittal EAM-C R Initial.

\begin{tabular}{lc}
\hline \multicolumn{1}{c}{ Statistic } & Value \\
\hline & \\
Session Two (mean) & 13.5917 \\
Session One (mean) & 13.4833 \\
Mean Difference & 0.1083 \\
Standard Error & 0.1138 \\
Upper 95\% & 0.3588 \\
Lower 95\% & -0.1421 \\
Sample size & 12 \\
Correlation & 0.9662 \\
Paired t test & 0.9520 \\
Degrees freedom & 11 \\
P value (2 tail) & 0.3615 \\
\hline
\end{tabular}


Table D-87. Assessment of intraobserver repeatability for the variable Sagittal EAM-P L Final.

\begin{tabular}{lc}
\hline \multicolumn{1}{c}{ Statistic } & Value \\
\hline Session Two (mean) & \\
Session One (mean) & 6.1167 \\
Mean Difference & 6.1167 \\
Standard Error & 0.0000 \\
Upper 95\% & 0.0508 \\
Lower 95\% & 0.1117 \\
Sample size & -0.1117 \\
Correlation & 12 \\
Paired t test & 0.9846 \\
Degrees freedom & 0.0000 \\
P value (2 tail) & 11 \\
\hline
\end{tabular}

Table D-88. Assessment of intraobserver repeatability for the variable Sagittal EAM-P L Initial.

\begin{tabular}{lc}
\hline \multicolumn{1}{c}{ Statistic } & Value \\
\hline & \\
Session Two (mean) & 5.9667 \\
Session One (mean) & 5.9000 \\
Mean Difference & 0.0667 \\
Standard Error & 0.0541 \\
Upper 95\% & 0.1858 \\
Lower 95\% & -0.0525 \\
Sample size & 12 \\
Correlation & 0.9884 \\
Paired t test & 1.2318 \\
Degrees freedom & 11 \\
P value (2 tail) & 0.2437 \\
\hline
\end{tabular}


Table D-89. Assessment of intraobserver repeatability for the variable Sagittal EAM-P R Final.

\begin{tabular}{lc}
\hline \multicolumn{1}{c}{ Statistic } & Value \\
\hline Session Two (mean) & \\
Session One (mean) & 6.4083 \\
Mean Difference & 6.3500 \\
Standard Error & 0.0583 \\
Upper 95\% & 0.0543 \\
Lower 95\% & 0.1778 \\
Sample size & -0.0612 \\
Correlation & 12 \\
Paired t test & 0.9875 \\
Degrees freedom & 1.0743 \\
P value (2 tail) & 11 \\
\hline
\end{tabular}

Table D-90. Assessment of intraobserver repeatability for the variable Sagittal EAM-P R Initial.

\begin{tabular}{lc}
\hline \multicolumn{1}{c}{ Statistic } & Value \\
\hline & \\
Session Two (mean) & 6.4167 \\
Session One (mean) & 6.2583 \\
Mean Difference & 0.1583 \\
Standard Error & 0.0398 \\
Upper 95\% & 0.2460 \\
Lower 95\% & 0.0707 \\
Sample size & 12 \\
Correlation & 0.9956 \\
Paired t test & 3.9775 \\
Degrees freedom & 11 \\
P value (2 tail) & 0.0022 \\
\hline
\end{tabular}


Table D-91. Assessment of intraobserver repeatability for the variable Sagittal FD L Final.

\begin{tabular}{lc}
\hline \multicolumn{1}{c}{ Statistic } & Value \\
\hline Session Two (mean) & \\
Session One (mean) & 8.0083 \\
Mean Difference & 8.0667 \\
Standard Error & -0.0583 \\
Upper 95\% & 0.0468 \\
Lower 95\% & 0.0447 \\
Sample size & -0.1613 \\
Correlation & 12 \\
Paired t test & 0.9855 \\
Degrees freedom & -1.2463 \\
P value (2 tail) & 11 \\
\hline
\end{tabular}

Table D-92. Assessment of intraobserver repeatability for the variable Sagittal FD L Initial.

\begin{tabular}{lc}
\hline \multicolumn{1}{c}{ Statistic } & Value \\
\hline & \\
Session Two (mean) & 7.5750 \\
Session One (mean) & 7.5417 \\
Mean Difference & 0.0333 \\
Standard Error & 0.0450 \\
Upper 95\% & 0.1323 \\
Lower 95\% & -0.0656 \\
Sample size & 12 \\
Correlation & 0.9906 \\
Paired t test & 0.7416 \\
Degrees freedom & 11 \\
P value (2 tail) & 0.4739 \\
\hline
\end{tabular}


Table D-93. Assessment of intraobserver repeatability for the variable Sagittal FD R Final.

\begin{tabular}{lc}
\hline \multicolumn{1}{c}{ Statistic } & Value \\
\hline Session Two (mean) & 7.6667 \\
Session One (mean) & 7.6250 \\
Mean Difference & 0.0417 \\
Standard Error & 0.0679 \\
Upper 95\% & 0.1912 \\
Lower 95\% & -0.1079 \\
Sample size & 12 \\
Correlation & 0.9685 \\
Paired t test & 0.6133 \\
Degrees freedom & 11 \\
P value (2 tail) & 0.5521 \\
\hline
\end{tabular}

Table D-94. Assessment of intraobserver repeatability for the variable Sagittal FD R Initial.

\begin{tabular}{lc}
\hline \multicolumn{1}{c}{ Statistic } & Value \\
\hline & \\
Session Two (mean) & 7.6250 \\
Session One (mean) & 7.5917 \\
Mean Difference & 0.0333 \\
Standard Error & 0.0711 \\
Upper 95\% & 0.1898 \\
Lower 95\% & -0.1231 \\
Sample size & 12 \\
Correlation & 0.9765 \\
Paired t test & 0.4690 \\
Degrees freedom & 11 \\
P value (2 tail) & 0.6482 \\
\hline
\end{tabular}


Table D-95. Assessment of intraobserver repeatability for the variable Sagittal FW L Final.

\begin{tabular}{lc}
\hline \multicolumn{1}{c}{ Statistic } & Value \\
\hline Session Two (mean) & \\
Session One (mean) & 16.5500 \\
Mean Difference & 17.8667 \\
Standard Error & -1.3167 \\
Upper 95\% & 1.2895 \\
Lower 95\% & 1.5215 \\
Sample size & -4.1549 \\
Correlation & 12 \\
Paired t test & 0.5263 \\
Degrees freedom & -1.0211 \\
P value (2 tail) & 11 \\
\hline
\end{tabular}

Table D-96. Assessment of intraobserver repeatability for the variable Sagittal FW L Initial.

\begin{tabular}{lc}
\hline \multicolumn{1}{c}{ Statistic } & Value \\
\hline Session Two (mean) & 17.9667 \\
Session One (mean) & 17.6500 \\
Mean Difference & 0.3167 \\
Standard Error & 0.1381 \\
Upper 95\% & 0.6206 \\
Lower 95\% & 0.0128 \\
Sample size & 12 \\
Correlation & 0.9855 \\
Paired t test & 2.2934 \\
Degrees freedom & 11 \\
P value (2 tail) & 0.0425 \\
\hline
\end{tabular}


Table D-97. Assessment of intraobserver repeatability for the variable Sagittal FW R Final.

\begin{tabular}{lc}
\hline \multicolumn{1}{c}{ Statistic } & Value \\
\hline Session Two (mean) & 18.6500 \\
Session One (mean) & 18.5417 \\
Mean Difference & 0.1083 \\
Standard Error & 0.1215 \\
Upper 95\% & 0.3758 \\
Lower 95\% & -0.1591 \\
Sample size & 12 \\
Correlation & 0.9596 \\
Paired t test & 0.8915 \\
Degrees freedom & 11 \\
P value (2 tail) & 0.3917 \\
\hline
\end{tabular}

Table D-98. Assessment of intraobserver repeatability for the variable Sagittal FW R Initial.

\begin{tabular}{lc}
\hline \multicolumn{1}{c}{ Statistic } & Value \\
\hline Session Two (mean) & 18.2500 \\
Session One (mean) & 18.2500 \\
Mean Difference & 0.0000 \\
Standard Error & 0.1161 \\
Upper 95\% & 0.2556 \\
Lower 95\% & -0.2556 \\
Sample size & 12 \\
Correlation & 0.9816 \\
Paired t test & 0.0000 \\
Degrees freedom & 11 \\
P value (2 tail) & 1.0000 \\
\hline
\end{tabular}


Table D-99. Assessment of intraobserver repeatability for the variable Sagittal MJS L Final.

\begin{tabular}{lc}
\hline \multicolumn{1}{c}{ Statistic } & Value \\
\hline Session Two (mean) & 2.3333 \\
Session One (mean) & 2.3417 \\
Mean Difference & -0.0083 \\
Standard Error & 0.0557 \\
Upper 95\% & 0.1142 \\
Lower 95\% & -0.1309 \\
Sample size & 12 \\
Correlation & 0.9459 \\
Paired t test & -0.1497 \\
Degrees freedom & 11 \\
P value (2 tail) & 0.8837 \\
\hline
\end{tabular}

Table D-100. Assessment of intraobserver repeatability for the variable Sagittal MJS L Initial.

\begin{tabular}{lc}
\hline \multicolumn{1}{c}{ Statistic } & Value \\
\hline Session Two (mean) & 2.1750 \\
Session One (mean) & 2.2000 \\
Mean Difference & -0.0250 \\
Standard Error & 0.0463 \\
Upper 95\% & 0.0768 \\
Lower 95\% & -0.1268 \\
Sample size & 12 \\
Correlation & 0.9810 \\
Paired t test & -0.5404 \\
Degrees freedom & 11 \\
P value (2 tail) & 0.5997 \\
\hline
\end{tabular}


Table D-101. Assessment of intraobserver repeatability for the variable Sagittal MJS R Final.

\begin{tabular}{lc}
\hline \multicolumn{1}{c}{ Statistic } & Value \\
\hline Session Two (mean) & \\
Session One (mean) & 2.5667 \\
Mean Difference & 2.5667 \\
Standard Error & 0.0000 \\
Upper 95\% & 0.0461 \\
Lower 95\% & 0.1014 \\
Sample size & -0.1014 \\
Correlation & 12 \\
Paired t test & 0.8559 \\
Degrees freedom & 0.0000 \\
P value (2 tail) & 11 \\
\hline
\end{tabular}

Table D-102. Assessment of intraobserver repeatability for the variable Sagittal MJS R Initial.

\begin{tabular}{lc}
\hline \multicolumn{1}{c}{ Statistic } & Value \\
\hline & \\
Session Two (mean) & 2.5333 \\
Session One (mean) & 2.5167 \\
Mean Difference & 0.0167 \\
Standard Error & 0.0423 \\
Upper 95\% & 0.1099 \\
Lower 95\% & -0.0765 \\
Sample size & 12 \\
Correlation & 0.9702 \\
Paired t test & 0.3936 \\
Degrees freedom & 11 \\
P value (2 tail) & 0.7014 \\
\hline
\end{tabular}


Table D-103. Assessment of intraobserver repeatability for the variable Sagittal PJS L Initial.

\begin{tabular}{lc}
\hline \multicolumn{1}{c}{ Statistic } & Value \\
\hline Session Two (mean) & \\
Session One (mean) & 2.275 \\
Mean Difference & 2.28333 \\
Standard Error & -0.0083 \\
Upper 95\% & 0.04516 \\
Lower 95\% & 0.09106 \\
Sample size & -0.1077 \\
Correlation & 12 \\
Paired t test & 0.98182 \\
Degrees freedom & -0.18454 \\
P value (2 tail) & 11 \\
\hline
\end{tabular}

Table D-104. Assessment of intraobserver repeatability for the variable Sagittal PJS L Final.

\begin{tabular}{lc}
\hline \multicolumn{1}{c}{ Statistic } & Value \\
\hline Session Two (mean) & 2.08333 \\
Session One (mean) & 2.09167 \\
Mean Difference & -0.0083 \\
Standard Error & 0.03786 \\
Upper 95\% & 0.07499 \\
Lower 95\% & -0.0917 \\
Sample size & 12 \\
Correlation & 0.99274 \\
Paired t test & -0.22013 \\
Degrees freedom & 11 \\
P value (2 tail) & 0.8298 \\
\hline
\end{tabular}


Table D-105. Assessment of intraobserver repeatability for the variable Sagittal PJS R Final.

\begin{tabular}{lc}
\hline \multicolumn{1}{c}{ Statistic } & Value \\
\hline Session Two (mean) & \\
Session One (mean) & 2.5083 \\
Mean Difference & 2.4750 \\
Standard Error & 0.0333 \\
Upper 95\% & 0.0498 \\
Lower 95\% & 0.1428 \\
Sample size & -0.0762 \\
Correlation & 12 \\
Paired t test & 0.9788 \\
Degrees freedom & 0.6701 \\
P value (2 tail) & 11 \\
\hline
\end{tabular}

Table D-106. Assessment of intraobserver repeatability for the variable Sagittal PJS R Initial.

\begin{tabular}{lc}
\hline \multicolumn{1}{c}{ Statistic } & Value \\
\hline & \\
Session Two (mean) & 2.5667 \\
Session One (mean) & 2.6417 \\
Mean Difference & -0.0750 \\
Standard Error & 0.0687 \\
Upper 95\% & 0.0762 \\
Lower 95\% & -0.2261 \\
Sample size & 12 \\
Correlation & 0.9758 \\
Paired t test & -1.0921 \\
Degrees freedom & 11 \\
P value (2 tail) & 0.2981 \\
\hline
\end{tabular}


Table D-107. Assessment of intraobserver repeatability for the variable Sellavertical-M Final.

\begin{tabular}{lc}
\hline \multicolumn{1}{c}{ Statistic } & Value \\
\hline Session Two (mean) & 48.9250 \\
Session One (mean) & 49.2083 \\
Mean Difference & -0.2833 \\
Standard Error & 0.2962 \\
Upper 95\% & 0.3685 \\
Lower 95\% & -0.9351 \\
Sample size & 12 \\
Correlation & 0.9874 \\
Paired t test & -0.9567 \\
Degrees freedom & 11 \\
P value (2 tail) & 0.3593 \\
\hline
\end{tabular}

Table D-108. Assessment of intraobserver repeatability for the variable Sellavertical-M Initial.

\begin{tabular}{lc}
\hline \multicolumn{1}{c}{ Statistic } & Value \\
\hline Session Two (mean) & 46.1250 \\
Session One (mean) & 46.3250 \\
Mean Difference & -0.2000 \\
Standard Error & 0.4902 \\
Upper 95\% & 0.8789 \\
Lower 95\% & -1.2789 \\
Sample size & 12 \\
Correlation & 0.8955 \\
Paired t test & -0.4080 \\
Degrees freedom & 11 \\
P value (2 tail) & 0.6911 \\
\hline
\end{tabular}


Table D-109. Assessment of intraobserver repeatability for the variable SNA Final.

\begin{tabular}{lc}
\hline \multicolumn{1}{c}{ Statistic } & Value \\
\hline Session Two (mean) & 81.4833 \\
Session One (mean) & 81.3083 \\
Mean Difference & 0.1750 \\
Standard Error & 0.2206 \\
Upper 95\% & 0.6605 \\
Lower 95\% & -0.3105 \\
Sample size & 12 \\
Correlation & 0.9809 \\
Paired t test & 0.7934 \\
Degrees freedom & 11 \\
P value (2 tail) & 0.4443 \\
\hline
\end{tabular}

Table D-110. Assessment of intraobserver repeatability for the variable SNA Initial.

\begin{tabular}{lc}
\hline \multicolumn{1}{c}{ Statistic } & Value \\
\hline Session Two (mean) & 81.8833 \\
Session One (mean) & 82.1333 \\
Mean Difference & -0.2500 \\
Standard Error & 0.1351 \\
Upper 95\% & 0.0474 \\
Lower 95\% & -0.5474 \\
Sample size & 12 \\
Correlation & 0.9902 \\
Paired t test & -1.8502 \\
Degrees freedom & 11 \\
P value (2 tail) & 0.0913 \\
\hline
\end{tabular}


Table D-111. Assessment of intraobserver repeatability for the variable SNB Final.

\begin{tabular}{lc}
\hline \multicolumn{1}{c}{ Statistic } & Value \\
\hline Session Two (mean) & 76.6167 \\
Session One (mean) & 76.5667 \\
Mean Difference & 0.0500 \\
Standard Error & 0.1964 \\
Upper 95\% & 0.4822 \\
Lower 95\% & -0.3822 \\
Sample size & 12 \\
Correlation & 0.9829 \\
Paired t test & 0.2546 \\
Degrees freedom & 11 \\
P value (2 tail) & 0.8037 \\
\hline
\end{tabular}

Table D-112. Assessment of intraobserver repeatability for the variable SNB Initial.

\begin{tabular}{lc}
\hline \multicolumn{1}{c}{ Statistic } & Value \\
\hline Session Two (mean) & 76.3500 \\
Session One (mean) & 76.4500 \\
Mean Difference & -0.1000 \\
Standard Error & 0.1128 \\
Upper 95\% & 0.1483 \\
Lower 95\% & -0.3483 \\
Sample size & 12 \\
Correlation & 0.9928 \\
Paired t test & -0.8864 \\
Degrees freedom & 11 \\
P value (2 tail) & 0.3944 \\
\hline
\end{tabular}




\section{VITA}

Brenton Edward Glassell, the son of Dr. Edwin and Deborah Glassell, was born in Fort Smith, Arkansas in 1984. Brenton graduated from Clarksville Academy in Clarksville, Tennessee, in 2002 and attended the University of Arkansas where he received a Bachelor of Science degree in Biology. He received his dental training and a Doctor of Dental Surgery degree from The University of Tennessee Health Science Center, Memphis, in May 2010. In August 2010, he entered The University of Tennessee Health Science Center as a graduate student in the Department of Orthodontics and is expected to receive his Master of Dental Science in May 2013. Brenton married his wife, Gail Glassell, in 2011. 